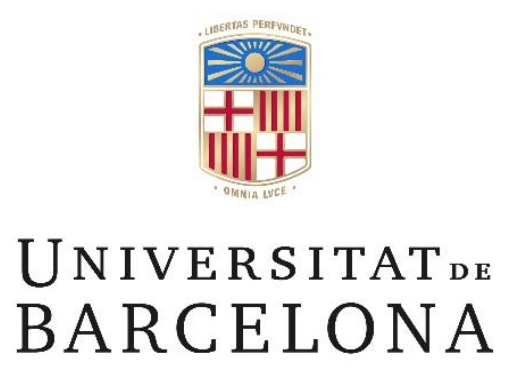

\title{
Mesoscopic Nonequilibrium Kinetics of Nucleation Processes
}

\author{
David Reguera López
}

\begin{abstract}
ADVERTIMENT. La consulta d'aquesta tesi queda condicionada a l'acceptació de les següents condicions d'ús: La difusió d'aquesta tesi per mitjà del servei TDX (www.tdx.cat) i a través del Dipòsit Digital de la UB (diposit.ub.edu) ha estat autoritzada pels titulars dels drets de propietat intel-lectual únicament per a usos privats emmarcats en activitats d'investigació i docència. No s'autoritza la seva reproducció amb finalitats de lucre ni la seva difusió i posada a disposició des d'un lloc aliè al servei TDX ni al Dipòsit Digital de la UB. No s'autoritza la presentació del seu contingut en una finestra o marc aliè a TDX o al Dipòsit Digital de la UB (framing). Aquesta reserva de drets afecta tant al resum de presentació de la tesi com als seus continguts. En la utilització o cita de parts de la tesi és obligat indicar el nom de la persona autora.
\end{abstract}

ADVERTENCIA. La consulta de esta tesis queda condicionada a la aceptación de las siguientes condiciones de uso: La difusión de esta tesis por medio del servicio TDR (www.tdx.cat) y a través del Repositorio Digital de la UB (diposit.ub.edu) ha sido autorizada por los titulares de los derechos de propiedad intelectual únicamente para usos privados enmarcados en actividades de investigación y docencia. No se autoriza su reproducción con finalidades de lucro ni su difusión y puesta a disposición desde un sitio ajeno al servicio TDR o al Repositorio Digital de la UB. No se autoriza la presentación de su contenido en una ventana o marco ajeno a TDR o al Repositorio Digital de la UB (framing). Esta reserva de derechos afecta tanto al resumen de presentación de la tesis como a sus contenidos. En la utilización o cita de partes de la tesis es obligado indicar el nombre de la persona autora.

WARNING. On having consulted this thesis you're accepting the following use conditions: Spreading this thesis by the TDX (www.tdx.cat) service and by the UB Digital Repository (diposit.ub.edu) has been authorized by the titular of the intellectual property rights only for private uses placed in investigation and teaching activities. Reproduction with lucrative aims is not authorized nor its spreading and availability from a site foreign to the TDX service or to the UB Digital Repository. Introducing its content in a window or frame foreign to the TDX service or to the UB Digital Repository is not authorized (framing). Those rights affect to the presentation summary of the thesis as well as to its contents. In the using or citation of parts of the thesis it's obliged to indicate the name of the author. 


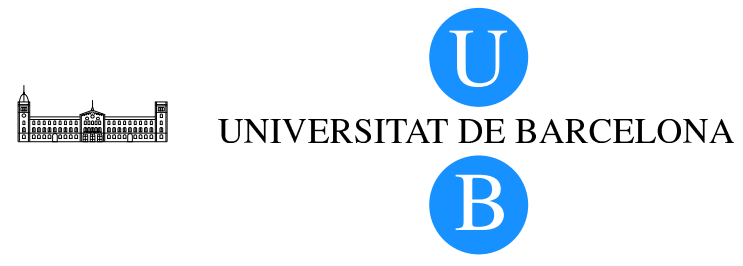

Facultat de Física

Departament de Física Fonamental

\section{Mesoscopic Nonequilibrium Kinetics of Nucleation Processes}

Programa de doctorado: Métodos Estadísticos de la Física Bienio 1997-1999

Memoria presentada por David Reguera López para optar al título de Doctor en Ciencias Físicas. 



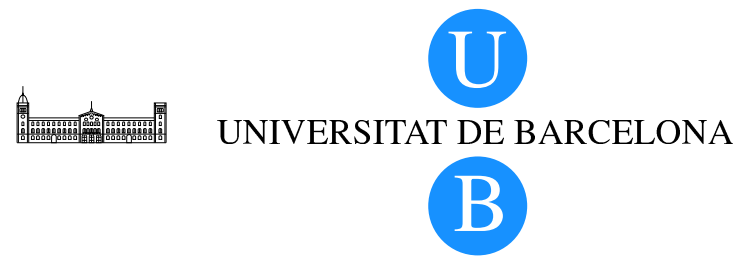

Facultat de Física

Departament de Física Fonamental

\section{Mesoscopic Nonequilibrium Kinetics of Nucleation Processes}

Certifico que la presente tesis doctoral ha sido realizada bajo mi dirección. Barcelona, Octubre, 2001.

José Miguel Rubí Capaceti Catedrático de Física de la Materia Condensada Departament de Física Fonamental Universitat de Barcelona 



\section{Agradecimientos}

Si ya resulta complicada la tarea de condensar en unas pocas páginas lo que ha sido tu trabajo en los últimos cuatro años, no lo es menos el hecho de dejar constancia de todas las personas que, de un modo u otro, han contribuido a llevar este trabajo a buen término, o lo han hecho más fácil y agradable.

En primer lugar quisiera darle las gracias de un modo especial a Miguel Rubí, mi director de tesis, que ha sabido combinar la libertad de acción con la más absoluta de sus disponibilidades para ejercer de guía en este complicado mundo de la investigación. Tampoco quisiera olvidarme de Agustín, que ha contribuido de una manera importante a la consecución de esta tesis, ni de la Generalitat de Catalunya, por el apoyo económico que me ha brindado.

Me gustaría hacer extensivo este agradecimiento a todo el personal del departamento, que siempre ha mostrado la mejor de las aptitudes para cualquier cosa necesaria, y en especial a Ignacio y Félix, con los que he tenido el placer de discutir de temas científicos y de todo tipo.

También guardo un recuerdo muy especial para Howard Reiss y su grupo, Vanita, Zaira, Norma, y todas las personas que tan bien me acogieron en los

Los Ángeles e hicieron que mi estancia allí fuera verdaderamente agradable y enriquecedora. Gracias también a Peter Hänggi y colaboradores, por su hospitalidad en Augsburg.

En un plano más personal, me gustaría agradecer a Napo su amistad y su ayuda durante todo este tiempo; a Xavi, que acabará catalanizándome, y con el que he salido a flote de múltiples situaciones; y a todos los compañeros de este y de otros departamentos (como Mateu, Eduard, Marta, Adan, Pep, Enric, David...) que han soportado estoicamente mi insistencia en reclutarles a la hora de ir a tomar el café.

Ya que hablo de reclutamientos, mención especial se merecen los miembros del Komando, ahora prácticamente disuelto por el exilio y las circunstancias: la Mariscala Marian, y su ahora hombre de confianza Marian; ese poeta bohemio que es Ivan; el cabo Francesc, desaparecido en combate; nuestro inigualable 
corneta jpozo; Tomás, compañero de tantas aventuras y que siempre ha sabido marcar el camino a seguir; o a los irreductibles Miquel, Juanjo y Paqui, inasequibles al desaliento y con los que he empezado de la mejor manera posible los fines de semana. Gracias también al cocinero y camareros de nuestro cuartel general, La Esquinica, a quienes tanto hemos hecho sufrir, por lo mucho que nos han hecho disfrutar.

Gente muy especial para mí son también Nuria, con quien he compartido tantos momentos agradables, y que con su sentido común y su fuerza vital ha contribuido a que mi vida sea mucho más estimulante; y Grego, Loli, Jose, Óscar y Roberto, que han estado siempre ahí durante todo este tiempo y son los mejores amigos que uno pueda tener. También quisiera expresar mi agradecimiento a Carlos, mi profesor de instituto, y uno de los principales causantes de que me encuentre en esta tesitura; a Albito y toda la gente de La Trapería, y a todos los amigos de Santas Martas que durante todos estos años me han ayudado a mantener el contacto con la realidad y con mis raíces. Y, en definitiva, a tantas y tantas personas como Emilio, Jordi, Sonia, Maite...(la lista sería inacabable) con los que he tenido la suerte de cruzarme y que han contribuido a enriquecer mi existencia.

Finalmente, me gustaría agradecer de una manera muy especial a toda mi familia, que siempre me ha apoyado incondicionalmente; a aquéllos que, como Urbano y Mili, se han convertido en una especie de segunda familia para mí; y sobre todo a Montse y Roberto, que me han respaldado y ayudado más allá de lo imaginable. Por último, el agradecimiento más sentido es para mis padres que con su empeño, su cariño y facilitándome siempre todos los medios a su alcance, son los máximos responsables de mi vida y de este trabajo. A ellos va dedicada esta tesis. 
A mis padres 



\section{Contents}

$\begin{array}{lr}\text { Introduction } & 1\end{array}$

1 Homogeneous Nucleation: Experiments, Theory and $\begin{array}{ll}\text { Simulations } & 11\end{array}$

1.1 Experimental Techniques . . . . . . . . . . . . . . . . 12

1.1.1 Diffusion Cloud Chambers . . . . . . . . . . . . . 12

1.1.2 Expansion Chambers . . . . . . . . . . . . . . . 14

1.1.3 Limitations of the Experiments . . . . . . . . . . . 18

1.2 Nucleation Theory . . . . . . . . . . . . . . . . . . . 20

1.2.1 Classical Nucleation Theory . . . . . . . . . . . 20

1.2.2 Phenomenological Modifications to the CNT . . . . . 29

1.2.3 Kinetic Models . . . . . . . . . . . . . . . . . . . 32

1.2.4 Field and Density Functional Approaches . . . . . . . . . 34

1.2.5 Molecular Theories . . . . . . . . . . . . . . . . . 35

1.3 Simulations . . . . . . . . . . . . . . . . 37

1.3.1 Evaluation of Nucleation Barriers . . . . . . . . . . . . 37

1.3.2 Evaluation of Nucleation Rates . . . . . . . . . . . 38

1.4 Conclusions . . . . . . . . . . . . . . . . . . . . 39

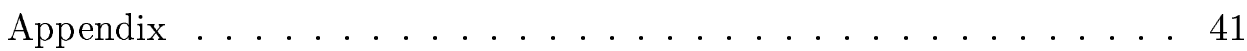

2 Mesoscopic Nonequilibrium Thermodynamics Description of Nucleation Kinetics $\quad 53$

2.1 Introduction . . . . . . . . . . . . . . . . 55

2.2 Mesoscopic Nonequilibrium Thermodynamics . . . . . . . . . 57

2.2.1 General Derivation . . . . . . . . . . . . . 57

2.3 MNET Approach to Nucleation Kinetics . . . . . . . . . . . . . 62

2.3.1 $\gamma$ is Related to the Number of Molecules in a Cluster . . 63

2.3.2 $\gamma$ Represents an Order Parameter . . . . . . . . . . . . . 64

$2.3 .3 \gamma$ is the Cluster Radius . . . . . . . . . . . 65 
2.3.4 $\gamma$ is the Set of Degrees of Freedom of the System . . . . 65

$2.3 .5 \gamma$ is the Density $\rho(\mathbf{r}) \ldots \ldots \ldots 66$

2.3.6 Hydrodynamic Description . . . . . . . . . . . 66 66

2.4 The Quasi-Stationary Case . . . . . . . . . . . . . . 69

2.5 Connection with Simulations . . . . . . . . . . 72

2.5.1 Quasi-Stationary Case . . . . . . . . . . . . 72

2.5.2 General Case . . . . . . . . . . . . . . 76

2.6 Conclusions . . . . . . . . . . . . . . . . . 77

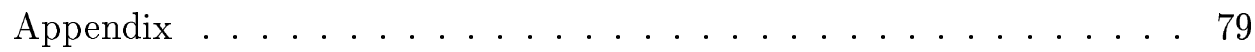

3 The Generalization of the Nucleation Theorem $\quad 87$

3.1 Introduction . . . . . . . . . . . . 88

3.2 Derivation of the Nucleation Theorem

from Small System Thermodynamics . . . . . . . . . . . . 91

3.3 Thermodynamic Proof of the Generalized Nucleation Theorem . 94

3.4 Nonuniform Density Distributions in a 1-D Hard Rod Fluid . . 100

3.4 .1 Initial Uniform State . . . . . . . . . . . . 100

3.4 .2 Cavity Formation . . . . . . . . . . . . . . . 101

3.4.3 Cluster Formation . . . . . . . . . . . . . . 105

3.5 The Nucleation Theorem and the Compressibility Equation of

State . . . . . . . . . . . . . . . . 108

3.6 The Nucleation Theorem and the Gibbs Adsorption Equation . . 109

3.7 The Nucleation Theorem and the Molecular Excess . . . . . . . 111

3.8 Conclusions . . . . . . . . . . . . . . . . . . . . . 113

4 Nonequilibrium Translational-Rotational Effects $\quad 117$

4.1 Influence of Translational-Rotational Degrees

of Freedom in Nucleation . . . . . . . . . . . . 118

4.1.1 Equilibrium Influence:

The Translational-Rotational Paradox . . . . . . . . . 119

4.1.2 Nonequilibrium Aspects: The Effects of Motion . . . . . . 123

4.2 Nonequilibrium Kinetics of Nucleation . . . . . . . . . . . . . 124

4.3 Nucleation Rate. . . . . . . . . . . . . . . . . . . 128

4.4 Conclusions . . . . . . . . . . . . . . . . . . . 131

5 Homogeneous Nucleation in Inhomogeneous Media 135

5.1 Nucleation under Spatially Inhomogeneous

Non-Isothermal Conditions . . . . . . . . . . . . . . 137

5.1.1 Homogeneous Nucleation in Spatially Inhomogeneous

Systems. Diffusion Regime . . . . . . . . . . . . 142 
5.1 .2 Temperature Evolution . . . . . . . . . . . . . 147

5.1.3 Influence of Diffusion and Thermal Diffusion in

Nucleation Experiments . . . . . . . . . . . . 149

5.1 .4 Conclusions . . . . . . . . . . . . . . . 160

5.2 Nucleation in a Shear Flow . . . . . . . . . . . . . . 161

5.2.1 Balance Equations in the Diffusion Regime . . . . . . . 165

5.2.2 Influence of the Shear Flow in Nucleation and

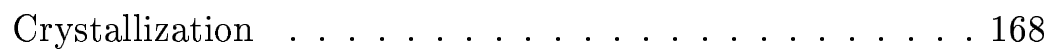

5.2 .3 Conclusions . . . . . . . . . . . . . . . . . . . . . . 169

Appendix . . . . . . . . . . . . . . . . . 170

6 Crystallization Beyond Nucleation $\quad 177$

6.1 The Polymer Crystallization Process . . . . . . . . . . . . . . . 179

6.2 Nucleation of Polyhedrical Clusters . . . . . . . . . . . . . . 180

6.3 Space-Filling in Terms of the Crystallinity Fraction . . . . . . . . 182

6.3.1 Avrami-Kolmogoroff Theory . . . . . . . . . . . . . 184

6.4 Kinetic Equations for the Crystallinity . . . . . . . . . . . . 187

6.4.1 Case 1: Isothermal Homogeneous Nucleation . . . . . . . 188

6.4.2 Case 2: Spatial Inhomogeneous Crystallization . . . . . 189

6.4.3 Case 3: Nucleation in a Shear Flow . . . . . . . . . . 189

6.5 Crystallization at Advanced Stages . . . . . . . . . . . . . . . 190

6.5.1 Interactions Between Clusters . . . . . . . . . . . . 191

6.5.2 Non-Conservative Equations . . . . . . . . . . . . . . 192

6.5.3 Anisotropic Effects . . . . . . . . . . . . . . . . . 192

6.6 Conclusions . . . . . . . . . . . . . . . . . 193

$\begin{array}{ll}\text { Conclusions and Perspectives } & 197\end{array}$

$\begin{array}{lr}\text { List of Publications } & 205\end{array}$ 



\section{Introduction}

Phase transitions are omnipresent phenomena in nature. The formation of liquid drops, bubbles or ice crystals in water are familiar manifestations of a process, which beyond its practical interest constitutes a fundamental problem in many areas of the scientific knowledge. The initial and crucial step in many of these phase transformations is the formation of small embryos or nucleus of the new phase within the bulk metastable substance. This fundamental mechanism of phase transformation is known as nucleation.

Basically, one can distinguish between two different types of nucleation: homogeneous nucleation which occurs in the bulk of a pure substance; and heterogeneous nucleation, which takes place in the presence of boundaries, surfaces, impurities or pre-existing particles. In fact, these walls and impurities provide preferential sites for the formation of the new phase and thus the heterogeneous nucleation is more common in many practical circumstances.

Nucleation phenomena appear in a wide variety of forms. Condensation (liquid drop formation in a supercooled gas), cavitation (bubble formation in a superheated liquid), crystallization or void formation occurring in simple systems, multicomponent mixtures, alloys or amphiphilic substances are fascinating examples of nucleation processes. But nucleation also plays a crucial role in very different areas of science and technology.

In atmospheric sciences, the nucleation of water droplets or ice crystals in the atmosphere is a fundamental issue in weather forecasting, and the cloud formation provoked by atmospheric aerosols (liquid droplets suspended in a gas) is one of the reasons of the global warming [1]. Nucleation can also be used to induce or prevent precipitations and thunderstorms by cloud seeding.

For pharmaceuticals, an appropriate choice of the crystal structure can enhance the bio-availability and effectiveness of a drug [2]. Examples of biological interest encompass the bio-mineralization of bone, teeth and shells [3]; the formation of kidney stones or uric acid crystals in the clinical condition of gout or protein crystallization [4]. This former example is specially important, since 
crystallization is the key to know the protein structure and from it, its functionality. In addition, protein crystallization is also implicated in certain diseases such as sickle-cell anemia and the formation of cataracts in the eye. In cryogenics, there is a strong interest to bypass and control nucleation of ice in the cryopreservation of embryos and human tissues [5].

The motor engine and turbine function, the oil and gas extraction, the fabrication of aerosols, coverings, or metallurgic materials constitute practical problems concerning relevant industries quite involved in the investigation and control over different aspects of nucleation.

Another area where nucleation constitutes a fundamental problem is geophysics. Nucleation is behind vulcanism [6] and planetary formation, and the understanding of earthquake nucleation [7],[8] is also a primal challenge to accomplish its predictability.

On the physical side, nucleation is for instance a major topic in the growth of low dimensional structures like quantum dots. Cavitation, nucleation of ${ }^{3} \mathrm{He}$, of vortex [9] in superconductors or of defects in materials [10] are also significant examples, which also embrace diverse applications to other areas as the modeling of traffic congestion and car jamming [11] thus revealing its multidisciplinary character. Nevertheless, the field where nucleation is probably most relevant is in the context of phase transformations in condensed matter systems and material science. Nucleation is deciding in manufacturing and in the development of new materials. An important requirement in the fabrication of advanced materials, such as polymers, ceramics or semiconductors is control over crystallization [12]. Precisely, the nucleation stage determines the rate, size, structure and purity of crystallization, which dictates the future properties of the material.

Despite all this clear interest arising from many disciplines, nucleation remains an important fundamental problem not completely understood. The reason for that fact lies in the peculiar characteristics of this phenomenon.

To illustrate the physical nature of the nucleation process let us consider a simple and well-known example: the condensation of a vapor. Figure 1 represents the pressure-volume phase diagram of a van der Waals gas. The solid line denotes the coexistence curve between the liquid and the vapor. The dashed line, represents the spinodal curve, which is the locus of points where the stability requirement of positive isothermal compressibility, $\kappa_{T}=-\frac{1}{V}\left(\frac{\partial V}{\partial p}\right)_{T}$, is first violated. Beneath the spinodal line, the vapor phase is thermodynamically unstable, and the phase transformation proceeds spontaneously by a barrierless mechanism known as spinodal decomposition. In the region between the spinodal and the coexistence lines, the system is metastable and the phase 

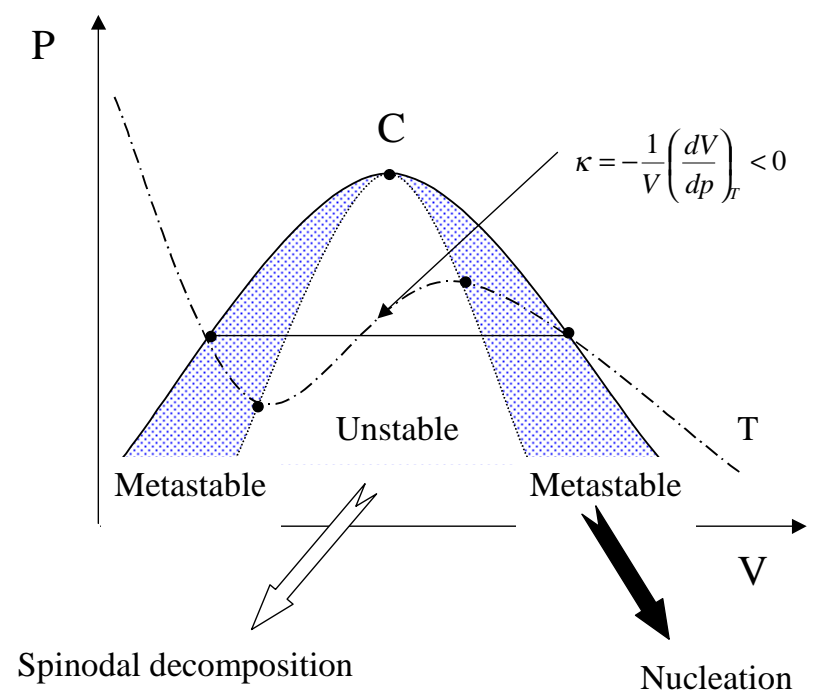

Nucleation
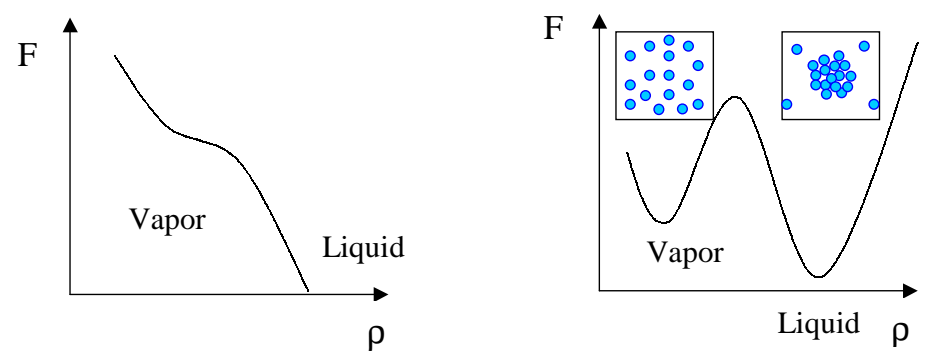

Figure 1: Phase diagram of a Van der Waals gas. The point $\mathrm{C}$ indicate the critical point. Coexistence (binodal) is shown as a full line, the spinodal curve with a dotted line, and the dashed-dotted line illustrates a typical isotherm. Below, the free energy is represented as a function of the density for the cases of spinodal decomposition and nucleation. 


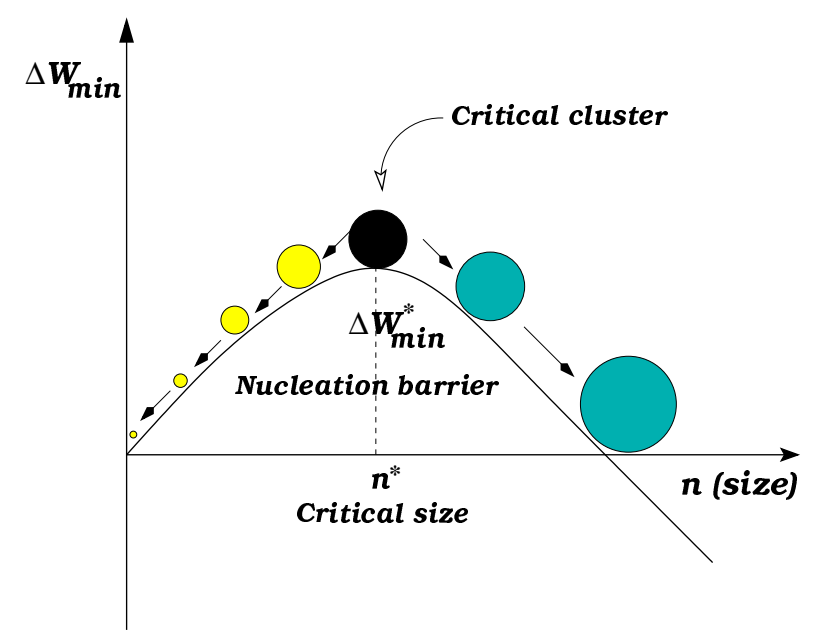

Figure 2: Plot of the energy $\Delta W_{\min }$ required to form a cluster of $n$ molecules, which illustrates the concepts of nucleation barrier $\Delta W_{m i n}^{*}$, critical cluster and critical size $n^{*}$.

transformation occurs by the nucleation and growth mechanisms.

The degree of metastability of a vapor is usually measured in terms of the supersaturation $S$, defined as $S=p / p_{e q}$ where $p$ is the actual pressure of the vapor and $p_{e q}$ is the equilibrium coexistence pressure. It is a well-known fact that a metastable phase can persists over long periods of time. For instance, a sample of purified water can be held indefinitely at $-10^{\circ} \mathrm{C}$ without freezing; or a gas can be compressed to several times its equilibrium pressure before the first liquid drop suddenly appears. This feature reflects the fact that the development of the new phase requires the surmounting of an energy barrier. This barrier becomes infinity at the coexistence curve and vanishes at the spinodal, thus indicating a strong dependency on temperature and supersaturation.

Let us analyze the physical origin of this barrier and how it is overcome. In the metastable phase, the thermal agitation of the molecules induces density fluctuations. These fluctuations generate small aggregates of molecules (also called clusters) with the same properties of the stable phase, in our case, tiny liquid droplets. The formation of these small droplets is favored by the energy decrease associated to the lower chemical potential of the stable liquid phase. However, the establishment of the new phase from the bulk metastable phase requires the creation of an interface between the two phases, which implies an energetic cost. The competition between these two effects is what originates the nucleation barrier, as illustrated in Fig. 2.

Surface effects are dominant for small clusters and hence tiny droplets tend 
to disintegrate. However, there exists a characteristic size beyond which volume effects overrides surface contributions and clusters tend to grow spontaneously. This size $n^{*}$ is known as the critical size and the energy required in its formation constitutes the nucleation barrier. The rate at which critical-sized embryos are formed is the nucleation rate $J$, and its prediction is one of the major goals of nucleation theories.

Although the first studies on phase equilibrium and metastability of undercooled substances date back to the investigations of Fahrenheit [13] in the eighteen century, the study of the kinetics of nucleation was initiated by the pioneering works of Volmer and Weber [14] in 1926, and Farkas in 1927 [15]. The field was subsequently developed by the contributions of Becker and Döring [16], Frenkel [17],[18] and Zeldovich [19], among others. These investigations constitute, collectively, the body of the Classical Nucleation Theory (CNT). This classical picture has dominated our understanding of the process during the last decades. The secret of that success lies in its striking simplicity and in the initial reasonable agreement of experimental results with its predictions for the metastability limits of the majority of substances.

In the last years, we have witnessed the sprouting of new and incredibly accurate experimental techniques able to measure actual nucleation rates. Moreover, for the first time the real-time experimental observation of the appearance and growing of nucleus [20] and small crystals at the nanoscale [4],[21], is becoming feasible. In parallel, simulations are increasingly becoming an impressive tool to characterize the rate of appearance and structure of nucleation events at the molecular scale [22]. These new findings, which provide an increasingly accurate exploration of the dynamics of the process at the mesoscale, have revealed the shortcomings of the Classical Nucleation Theory and impelled a renewed interest in the field.

Despite the significant effort in the subject, several important questions remain open. The most crucial one, is of course, that current nucleation theories are in general still unable to predict accurately the experimental values of homogeneous nucleation rate. The activated nature of the process, which implies that the rate depends exponentially on the barrier, makes the process extraordinarily sensitive to small variations of the parameters involved, and thus very difficult to control and measure. Nucleation is one of the few areas in science in which differences between predicted and measured values of nucleation rates within several orders of magnitude can in principle be considered as acceptable.

The classical nucleation theory uses macroscopic and equilibrium arguments to describe a mesoscopic nonequilibrium process as nucleation is. The approach to the problem from this perspective has been the cause of some long-standing 
controversies and of the misunderstanding of some key concepts. In addition, as the modern experimental and simulation techniques are evidencing, this simplified scheme is becoming insufficient to provide an accurate characterization of the process.

Since the free energy barrier is the dominant factor in the nucleation rate, most theoretical work has been focused on calculating the equilibrium energy of formation of the critical nucleus, quite disregarding the importance of nonequilibrium aspects of the process. One does not have to forget that nucleation is in essence a kinetic process, and in this sense nonequilibrium effects are unavoidable and could be relevant in its description.

Consequently, at the present state of the field it becomes very important to develop a framework for the description of nucleation and growth processes which should be as realistic as possible, taking into account its two distinctive characteristics: the fact that the process occurs at the mesoscopic scale and its intrinsic nonequilibrium nature. In addition, the prominent role that simulations are playing requires that this scheme should be somehow "friendly" providing a clear interpretation and connection with simulation results.

\section{Scope of the Thesis}

The purpose of the present thesis is to introduce a mesoscopic nonequilibrium description of the kinetics of nucleation processes. We shall present a consistent and complete framework to describe the nucleation kinetics which facilitates a more realistic and accurate description, retaining the potential nonequilibrium influences and the mesoscopic particularities of the process. Our formalism can be easily complemented with the results of previous theories, overcoming some of their drawbacks, and setting up a link with simulations.

We will focus our attention on first order phase transitions which proceed by homogeneous nucleation, essentially far from the critical point. Most of our analysis is intended for simple substances and gas-liquid systems. Nevertheless, nearly all the concepts discussed in this context remain valid for crystallization, and can be easily extended to more complex situations.

The content of the present work has been organized in chapters trying to focus on different and complementary aspects of nucleation kinetics.

The first chapter is intended as a brief review of the classical approach to the nucleation problem, including the most recent developments of experimental techniques, simulations and theories. This analysis shows the state-of-the-art

in the field and stress the necessity of an improved description of the process centered on nonequilibrium aspects. 
In Chapter 2, we establish the basis for a kinetic description of nucleation by proposing a mesoscopic formalism able to derive kinetic equations for the probability distribution functions. These functions may depend on all relevant parameters specifying the state of a cluster, i.e. geometrical parameters as the size or the geometry, phase space variables as the velocity, orientation, spatial position, etc., and their evolution is in general coupled to that of the environment in which the emerging clusters are embedded. The nucleation theory we propose thus goes beyond the classical formalism and can account systematically for the real conditions in which nucleation takes place.

A distinctive feature of many nucleation processes is its mesoscopic trait. The extrapolation of macroscopic concepts to the mesoscopic scale has precisely been the source of intense discussion and confusion in the field. One of the situations in which this fact becomes more evident is in the context of the socalled "Nucleation Theorem". In Chapter 3, we shall discuss the meaning of this theorem and demonstrate its general validity on purely thermodynamics grounds. In addition, we will outline how the proper understanding of the theorem from the mesoscopic perspective may establish the basis of a meaningful definition of cluster in nucleation.

The usefulness of a mesoscopic nonequilibrium description of nucleation becomes evident in the topic of Chapter 4. One of the most controversial aspects of nucleation theories has been and is the proper accounting for translational and rotational degrees of freedom. Within the framework developed in Chapter 2 , we will clarify this issue, and we shall obtain a new genuine nonequilibrium correction to the nucleation rate arising from the proper consideration of those degrees of freedom in the kinetics of the process. The correction we have found is significant and under some circumstances is able to achieve a remarkable agreement of the theoretical predictions with experiments.

This proper introduction of the translational and rotational degrees of freedom constitutes the first step toward a more realistic description of nucleation, retaining potential nonequilibrium influences. The metastable phase in which the embryos of the new phase are embedded may play a relevant role in the process and its influence cannot be ignored. In Chapter 5, we shall develop a general formalism able to describe the potential influence of the fluid in the process, focusing on typical situations of practical interest. In particular, we will discuss some specific examples to describe nucleation and growth in spatially inhomogeneous systems or in the presence of external temperature gradients. Moreover, we will analyze a less studied but specially interesting case: nucleation under the influence of an external flow.

In our goal to provide a more realistic description of the phase transfor- 
mation, we will sketch in Chapter 6 how the process continues beyond the nucleation stage. We will show how the subsequent step of the phase transition, the growth and the space filling by the nucleated droplets, can be embraced in the same consistent nonequilibrium framework, capable of a more complete characterization of the process.

Finally, we summarize the main conclusions of our work, and discuss some of our findings which will constitute the basis for subsequent developments. 


\section{Bibliography}

[1] M. Kulmala, U. Pirjola, and J.M. Makela, Nature 404, 66 (2000).

[2] S. R. Byrn, Solid State Chemistry of Drugs, (Academic Press, New York, 1982).

[3] S. Mann and G.A. Ozin, Nature 382, 313 (1996).

[4] S.-T. Yau and P.G. Vekilov, Nature 406, 494 (2000).

[5] R.E. Lee, G.J. Warren, and L.V. Gusta, Biological Ice Nucleation and Its Applications, (APS Press, 1995).

[6] A.W. Woods and S.S. Cardoso, Nature 385, 518 (1997).

[7] R.S. Stein, Nature 402, 605 (1999).

[8] W.L. Ellsworth and G.C. Beroza, Science 268, 851 (1995).

[9] See for instance: A. Soibel et al., Nature 406, 282 (2000).

[10] A. Gouldstone, K.J. Van Vliet, and S. Suresh, Nature 411, 656 (2001).

[11] J. Kaupuzs and R. Mahnke, AIP Conf. Proc. 534, 221 (2000).

[12] J. Aizenberg, A.J. Black, and G.M. Whitesides, Nature 398, 495 (1999).

[13] D.B. Fahrenheit, Phil. Trans. Roy. Soc. 39, 78 (1724). See also: M. Volmer, Kinetic der Phasenbildung (Theodor Steinkopff, Dresden, 1939), translated in English by Intelligent Department , AMC, for a good historical survey.

[14] M. Volmer and A. Weber, Z. Phys. Chem. 119, 277 (1926).

[15] L. Farkas, Z. Phys. Chem. 125, 236 (1927). 
[16] R. Becker and W. Döring, Ann. Phys. 24, 719 (1935).

[17] J. Frenkel, J. Chem. Phys. 7, 538 (1939).

[18] J. Frenkel, Kinetic Theory of Liquids, (Dover, New York, 1955).

[19] Y.I. Zeldovich, Zh. Eksp. Teor. Fiz 12, 525 (1942).

[20] U. Gasser, E.R. Weeks, A. Schofield, P.N. Pusey, and D.A. Weitz, Science 292, 258 (2001).

[21] D.W. Oxtoby, Nature 406, 464 (2000).

[22] S. Auer and D. Frenkel, Nature 409, 1020 (2001). 


\section{Chapter 1}

\section{Homogeneous Nucleation: Experiments, Theory and Simulations}

There has been a pronounced historical interest in the study of nucleation, starting from the early investigations of Fahrenheit [1] about metastability up to the most recent and impressive progress in simulations [2] and experimental techniques [3]. The core of the classical treatment of nucleation was essentially developed in the twenties and thirties [4]-[9], but investigations continued steadily until the recent spectacular resurgence of the field commanded by the puzzling findings of experiments and simulations. As a consequence, a huge bibliographic background on the subject exists and the task of providing a comprehensive and meticulous review of all contributions is really difficult and exceeds the scope of this thesis. Besides, very good reviews on the topic can be found in the literature [10]-[16].

Our purpose in this chapter will be to present the state-of-the-art in the field of homogeneous vapor phase nucleation. Since experiments are playing a crucial role in the recent advances in the subject, we shall start with a short survey about the more customary experimental techniques. We will then present a brief compendium of the different theoretical approaches proposed up to now to analyze homogeneous nucleation, including some of the most recent topics and developments. Finally, we will review the contributions arising from simulations, to conclude with a short summary.

Although the review will be focused on gas-liquid homogeneous nucleation, many of the theories and concepts discussed here, and in particular the Classical 
Nucleation Theory (CNT), are also valid for crystal nucleation, as mentioned in Sec. 1.2.1.

\subsection{Experimental Techniques}

Traditional experimental techniques of nucleation from the vapor phase only were able to detect the onset of nucleation, by measuring the values of the critical supersaturation $S_{c r}$ as a function of the temperature. The $S_{c r}$ is defined as the value of the supersaturation required to obtain an observable droplet formation, typically with a rate of $J_{c r} \approx 1 \mathrm{~cm}^{-3} \mathrm{~s}^{-1}$. These measurements could then be compared with the predictions of the Classical Nucleation Theory, and the agreement was initially quite correct for the vast majority of substances. However, critical supersaturation measurements do not provide a very sensitive check of the accuracy of the theories, since small discrepancies in $S_{c r}$ correspond, due to the exponentially activated character of the process, to errors in the nucleation rates of several orders of magnitude.

In the last years, new experimental techniques have been developed which are able to measure actual nucleation rates, instead of just critical supersaturations. These experiments have stimulated the interest in the field since they constitute a more stringent test which has revealed the shortcomings of CNT.

It is not our aim in this section to provide an extensive review of recent experiments. (A comprehensive list of experiments performed during the period comprised between 1968 and 1992 can be found in Ref. [17], and earlier experiments have been reported in Ref. [18]). However, the prominent role that experiments have played in the revitalization of the field of nucleation certainly deserves some revision. We also want to stress out the difficulties and the particular conditions under which nucleation rates are experimentally measured, and discuss the real accuracy and reliability of the measurements.

According to the procedure employed to generate the supercooled conditions in which nucleation occurs, we can roughly classify the experiments into two big families: diffusion and expansion experiments. These techniques are discussed in more detail below.

\subsubsection{Diffusion Cloud Chambers}

In diffusion chambers nucleation is accomplished by means of the establishment of inhomogeneous thermodynamic conditions which generate a supersaturation profile inside the chamber. Nucleation then occurs in the region, usually quite narrow, where supersaturation exceeds the critical value. Therefore, this proce- 
dure generates stationary but spatially inhomogeneous nucleation events. The main difficulty of these experimental devices is that require a quite complicated modeling of the transport inside the chamber. Besides, the real conditions under which nucleation is taking place cannot be measured but are obtained instead from the solution of the transport equations.

The supersaturation profile which activates the nucleation can be rendered either by thermal diffusion, through the imposition of a temperature gradient as in the thermal diffusion chambers; or by cooling the nucleating gas with a flow, as occurs in the laminar flow diffusion chambers. An insight to both methods is given below.

\section{Thermal Diffusion Cloud Chambers}

The upward thermal diffusion cloud chamber, depicted schematically in Fig. 1.1 , is the most traditional instrument to investigate nucleation. A detailed description of the device and its operation can be found for instance in Refs. [19]-[24]. Initially it was only able to measure critical supersaturation as a function of temperature, but recently it has been used to measure actual nucleation rates in many substances (see for instance Refs. [25]-[31]). In this experimental device, a stationary temperature gradient is established between a warm pool of liquid on the bottom and a cooled upper surface. The liquid on the lower plate evaporates and the vapor diffuses upward through an inert gas establishing inside the chamber a temperature and supersaturation profile as the ones represented in Fig. 1.1. The temperature gradient can be adjusted in order that the supersaturation maximum, which typically occurs at $3 / 4$ of the chamber height, exceeds the critical value, thus initiating homogeneous nucleation. The nucleated drops then grow and fall down through the chamber. A laser beam near the bottom of the chamber finally detects and counts the falling droplets, thus providing a measure of their formation rate. Typical nucleation rates measured in these experiments range from $10^{-4}$ to $10^{3} \mathrm{~cm}^{-3} \mathrm{~s}^{-1}$.

One of the main inconveniences of this type of experiments is that the temperature and supersaturation profiles are not measured but have to be calculated by solving the complicate set of equations describing the heat and mass transport in the chamber. Therefore the accuracy of the results strongly depends on the precision of the thermophysical properties of the gas, and can be dramatically modified if the transport is altered due, for instance, to convection or condensation at the walls. In addition, the measurements are known to be affected by the value of the total pressure inside the chamber and by the nature of the carrier gas. Thorough studies of the transport in this chamber and po- 


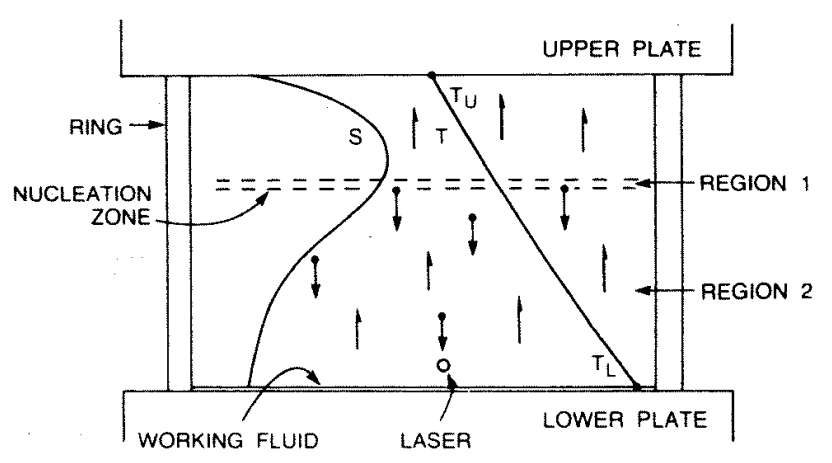

Figure 1.1: Schematic picture of an upward thermal diffusion cloud chamber, including the temperature $T$ and supersaturation $S$ profiles (from Ref. [26]).

tential effects altering nucleation rates measurements have been performed in the literature [32]-[40].

\section{Laminar Flow Diffusion Cloud Chambers}

The operational principle of the laminar flow diffusion chamber, illustrated in Fig. 1.2, is based on the sudden cooling of the nucleating gas assisted by a flow. A flowing carrier gas is initially saturated of the nucleating vapor (in the saturator) and then led to a cooling zone (the condenser) where the temperature suddenly drops. The vapor then becomes supersaturated and nucleation takes place. The drops are then removed from the nucleation region by the flow and subsequently grow until they can be detected optically. Also in this case the supersaturation and temperature profiles have to be calculated by solving the transport inside the chamber. Nucleation rates between $10^{2}$ to $10^{8} \mathrm{~cm}^{-3} \mathrm{~s}^{-1}$, can be measured in a wide range of supersaturations and temperatures [41][46]. However, the peculiarities of the operational principle of this chamber restrict the number of substances whose nucleation can be measured with this instrument. The laminar flow diffusion chamber is best suited for substances of high molecular weight, since the nucleation region becomes well defined only when the ratio of molecular to thermal diffusivity of the vapor is low.

\subsubsection{Expansion Chambers}

A different technique to measure nucleation rates is based on the cooling of the vapor through a fast expansion. The gas is suddenly expanded and hence cooled down achieving a supersaturated state in which nucleation takes place. 


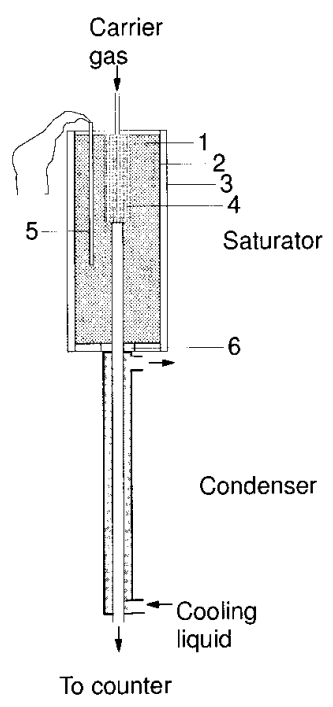

Figure 1.2: Scheme of a laminar flow diffusion chamber (from Ref. [44]).

After a short interval, typically a fraction of a second, the gas is recompressed, preventing the formation of additional nuclei but not the subsequent grow of the nucleated embryos, which are finally detected by optical means.

An important asset of this procedure is that, contrarily to the case of diffusion chambers, spatially homogeneous nucleation occurs, although it is not stationary. The use of this technique is thus restricted to substances and conditions for which the steady state value of the nucleation rate is achieved very rapidly. Nevertheless, this method is quite versatile, and has as main advantages that the supersaturation pulses are well defined, and the values of the pressure and the corresponding supersaturation can be experimentally measured without depending on the solution of a complicate modeling as happens in diffusion chambers. As a counterpart, the value of the temperature at which nucleation occurs cannot be measured, but is calculated through the use of the Poisson law of adiabatic expansions. This law was formulated for equilibrium quasistatic situations, hence its applicability to a nonequilibrium fast expansion is questionable, especially in the shock tube device described below, where a shock-wave is employed to generate an extremely short-time nucleation pulse.

The different implementations of this technique are described below. 

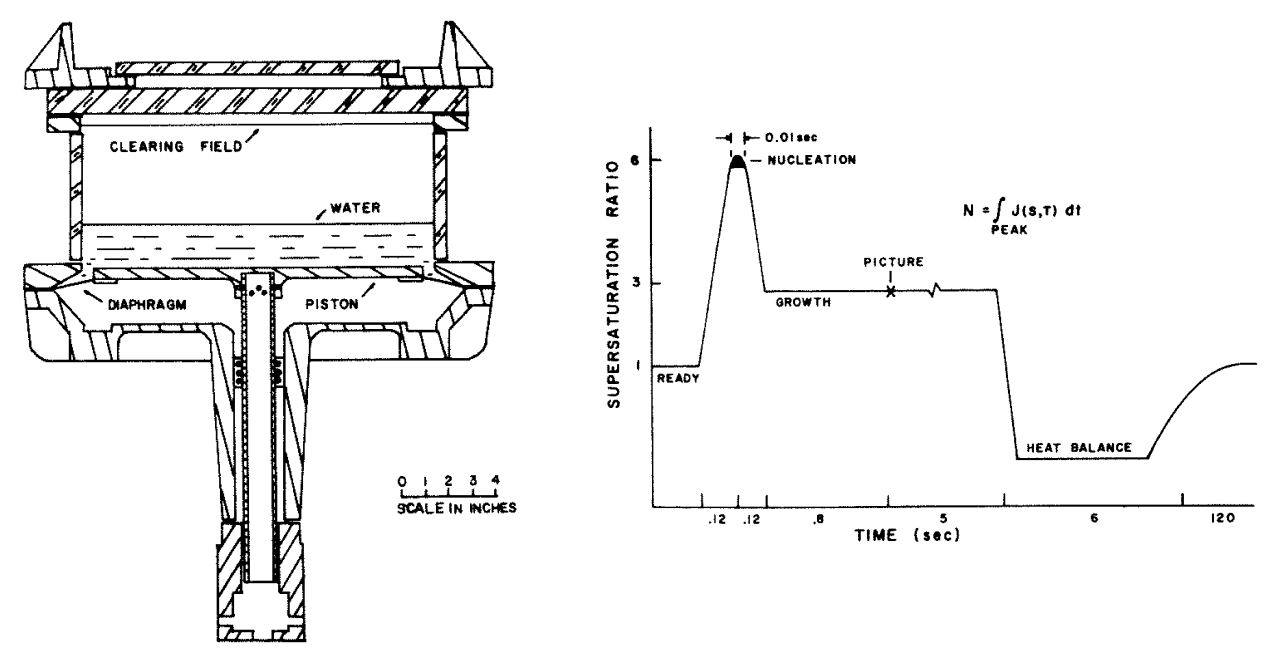

Figure 1.3: Basic components of a fast expansion chamber (left) and typical supersaturation ratio $S$ vs. time profile (right) (from Ref. [49]).

\section{Fast Expansion Chambers}

In the fast expansion cloud chamber, which is schematically drawn in Fig. 1.3, the vapor originates from the liquid pool resting on a piston at the bottom of the chamber. The piston is then displaced to provoke an adiabatic expansion of the gas, which becomes supersaturated and nucleates. A small recompression of the piston ends nucleation after typically $10 \mathrm{~ms}$, but the nucleated droplets are allowed to grow until they can be counted photographically. Figure 1.3 also shows the typical operation cycle in these chambers. Nucleation rates in the range of $10^{2}$ to $10^{5} \mathrm{~cm}^{-3} \mathrm{~s}^{-1}$ have been measured for several substances [47]-[51]. The main inconvenience of this method is that the measured nucleation rates are isoentropic rather than isothermal.

\section{Göttingen or Two-Piston Expansion Chamber}

A refined version of the expansion chamber is the two-piston or Göttingen chamber [52]-[59], schematically shown in Fig. 1.4. The presence of two pistons or valves improves the quality of the expansions and recompressions, achieving more defined and faster (on the order of $1 \mathrm{~ms}$ ) pressure profiles, as shown in Fig. 1.4. The counting of the nucleated droplets is also improved by using constant-angle Mie light scattering. Measurements have been performed on several substances in a rate interval of $10^{5}$ to $10^{10} \mathrm{~cm}^{-3} \mathrm{~s}^{-1}$. Recent developments 

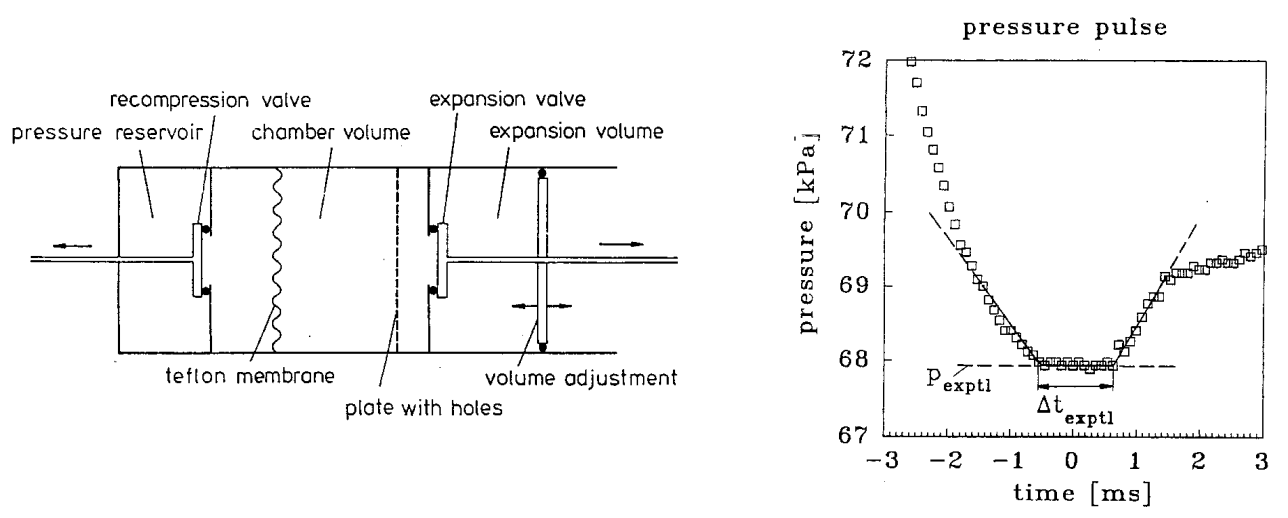

Figure 1.4: Cross-section of a two-piston expansion chamber (left) and detailed representation of a typical pressure pulse (right). The nucleation rate is calculated dividing the number density of observed droplets by $\Delta t_{e x p}$ (from Ref. [55]).

of design of the chamber [60] allow the measurement of nucleation rates keeping constant the nucleation temperature. This feature facilitates the comparison of the experimental results with theoretical predictions through the use of the Nucleation Theorem. As will be explained in Chapter 3, this theorem provides an excellent tool to test the molecular content of the nuclei independently of any particular theory.

\section{Shock Tubes}

Another implementation of the expansion method is the shock tube [61]-[64], shown in Fig. 1.5. In this device, a high pressure section (HPS) containing a vapor-inert gas mixture is separated from a low-pressure section (LPS) by a membrane. When the membrane is broken, an expansion wave travels in the direction of the HPS end-wall, causing the supersaturation of the vapor and the onset of nucleation. At the same time, a shock wave traveling in the LPS reflects on a constriction and causes the recompression of the gas, stopping the nucleation after an interval of $0.1 \mathrm{~ms}$. The number of drops nucleated during this short pulse can then be determined by the light intensity of constant-angle Mie scattering. High values of nucleation rates, from $10^{8}$ to $10^{11} \mathrm{~cm}^{-3} \mathrm{~s}^{-1}$, can be measured with this technique.

Finally, we want to just mention a different device which can reach even higher nucleation rates: the supersonic nozzle [65]-[68]. Unlike the other techniques, supersonic nozzles cannot directly measure nucleation rates, but they 


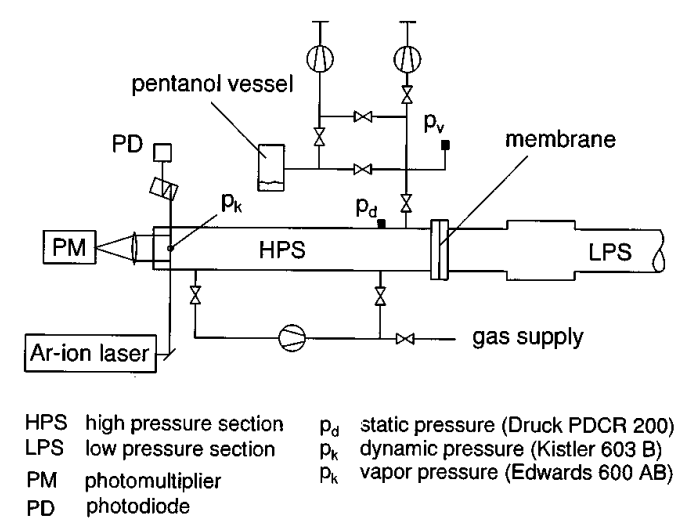

Figure 1.5: Schematic shock-wave tube setup used for nucleation experiments (from Ref. [63]).

are only able to detect the conditions for the onset of nucleation. Nevertheless, the relevance of this technique relays on the fact that it can reach extremely high supersaturations leading to the formation of critical clusters of molecular size, i.e 5-10 molecules. This device can then provide a very sensitive test to explore the validity of nucleation theories at molecular scale.

In both devices mentioned above, thermodynamic relations for adiabatic expansions have been employed to estimate the temperature at which nucleation is taking place. However, the extremely fast nature of the expansions raises some concerns about the validity of these equilibrium thermodynamic relations to extremely far from equilibrium processes such as shock-waves or supersonic expansions.

\subsubsection{Limitations of the Experiments}

A great progress has been achieved with the direct measurement of nucleation rates, and experimental techniques are becoming increasingly accurate. However, precise nucleation experiments are in nature extremely difficult to perform due to the utmost sensitivity of the nucleation rate to many factors, including the thermodynamic conditions or the presence of impurities. In this context, the comparison between theory and experiments must be taken with care, since significant disagreement persists even between experiments themselves. There exists also discrepancies between experiments performed employing different methods using the same substance or still when the same technique is applied by different experimentalists. Moreover, the same procedure applied at different 


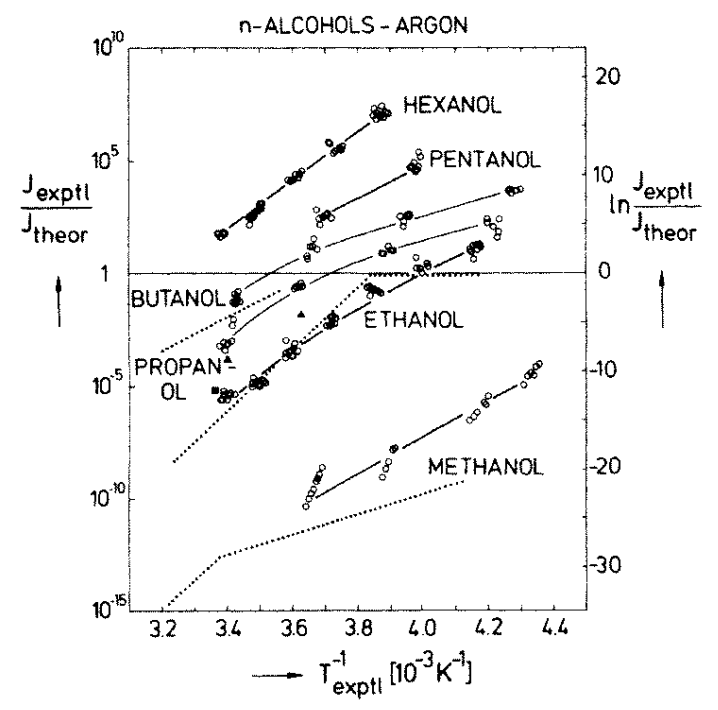

Figure 1.6: Comparison of experimental, $J_{\text {exptl}}$, and theoretical nucleation rates, $J_{t h e o r}$, predicted by Classical Theory vs. the inverse of the experimental temperature, $T_{\text {exptl }}$, for the homolog series of $n$-alcohols (from Ref. [53]).

conditions often provides incompatible results. To overcome this confusing situation, a significant effort has been recently done in order to create a common platform for comparing nucleation rate data measured with different techniques for the same substance [63],[57],[31],[46]. However, strong deviations of two or three orders of magnitude between different experimental results persist, which provide a useful hint about the real accuracy and reliability of the experimental measurements.

The different techniques described previously have been applied to measure nucleation rates for many substances and in a wide range of conditions, embracing many orders of magnitude of variation of the rate. In spite of the quantitative discrepancies, the general trend is that the variation of the nucleation rate with supersaturation predicted by CNT is approximately correct, but that the temperature dependence is not. The nucleation rates predicted by CNT are usually too low at low temperatures and too high at high temperatures, with errors with span several orders of magnitude in either direction, as is evidenced by Fig. 1.6 for the homogeneous nucleation of alcohols. These deviations of the CNT have stimulated the search for improved theories of nucleation. These theories, including the CNT, are summarized in the following section. 


\section{$1.2 \quad$ Nucleation Theory}

We can basically classify the different theories developed to study liquid-vapor nucleation into three categories. Phenomenological theories, as the classical nucleation theory (CNT), have the main objective of evaluating the free energy of formation of clusters by using macroscopic concepts such as the surface tension or the bulk density. In kinetic theories, the efforts are focused on the direct calculation of the rate coefficients controlling the growth or shrinking of the nucleated droplets. Finally, molecular, density functional theories, and even simulations can be grouped under the common label of microscopic models, since they pursue the understanding of the process directly from the microscopic dynamics and the intermolecular forces acting among molecules. We discuss and summarize all these approaches below.

\subsubsection{Classical Nucleation Theory}

The objective of a nucleation theory is the description of the evolution of the population of clusters of the new phase. In a supercooled substance, small embryos (or clusters) of the new stable phase are constantly being created and destroyed from density fluctuations. These clusters are assumed to shrink and grow in size by gaining or loosing single molecules. Consequently, the variation

of the population of clusters of size $n$ at each instant $t$ is given by the following master equation

$$
\begin{aligned}
\frac{\partial f(n, t)}{\partial t} & =k^{+}(n-1) f(n-1, t)+k^{-}(n+1) f(n+1, t) \\
& -k^{+}(n) f(n, t)-k^{-}(n) f(n, t),
\end{aligned}
$$

where $f(n, t)$ is the number density of clusters constituted by $n$ monomers at time $t ; k^{+}(n)$ is the rate at which a $n$-cluster gains monomers; and $k^{-}(n)$ is the rate at which it loses particles. This equation can be rewritten as

$$
\frac{\partial f(n, t)}{\partial t}=J(n-1, t)-J(n, t)
$$

where

$$
J(n) \equiv k^{+}(n) f(n, t)-k^{-}(n+1) f(n+1, t)
$$

is the net rate at which clusters of size $n$ become clusters of size $n+1$ at time $t$. 
The basic variables in the previous master equation (1.1) are $k^{+}(n)$ and $k^{-}(n)$, the rates of attachment and detachment of single molecules, respectively. The value of the condensation rate $k^{+}(n)$ is usually taken from kinetic theory of gases [12]. Assuming that every molecule which collides with a cluster sticks to it, the attachment rate is then given by the number of collisions with monomers that a cluster suffers per unit time

$$
k^{+}(n)=\frac{p}{\sqrt{2 \pi k_{B} T m_{1}}} A(n)\left(1+n^{-1 / 3}\right)^{2}\left(1+n^{-1}\right)^{1 / 2},
$$

where $p$ is the pressure due to single monomers, $T$ is the temperature, $k_{B}$ is the Boltzmann's constant, $m_{1}$ is the mass of a single molecule, and $A(n)=$ $s_{1} n^{2 / 3}$ is the surface area of a spherical cluster containing $n$ molecules, being $s_{1}=\left(36 \pi v_{1}^{2}\right)^{1 / 3}$ the surface area of a monomer and $v_{1}$ the average volume per molecule in the bulk liquid. The last two factors of the previous expression are corrections arising from the consideration of the surface of collision as spherical rather than flat, and from the translational motion of the clusters, respectively. However, these corrections are usually negligible [12] and the commonly used expression for $k^{+}(n)$ is simply

$$
k^{+}(n)=\frac{p}{\sqrt{2 \pi k_{B} T m_{1}}} s_{1} n^{2 / 3} .
$$

Unlike the rate $k^{+}(n)$, which can be obtained from first principles (using for instance kinetic theory), the value of the detachment rate $k^{-}(n)$ is more difficult to obtain in an independent way. To avoid this difficulty, phenomenological theories, as the classical nucleation theory, resort to an assumption referred to as constrained equilibrium hypothesis to obtain a relationship between the known quantity $k^{+}(n)$ and the unknown $k^{-}(n)$. This assumption is based on the fact that, at equilibrium, the flux $J(n)$ must vanish. As a consequence, from equation (1.3) one obtains the expression

$$
k^{+}(n) f_{e q}(n)-k^{-}(n+1) f_{e q}(n+1)=0 ; k^{-}(n+1)=\frac{f_{e q}(n)}{f_{e q}(n+1)} k^{+}(n),
$$

where $f_{e q}(n)$ represents the equilibrium distribution of clusters. Therefore, the kinetic problem of calculating the nucleation rate becomes the thermodynamic problem of evaluating the equilibrium cluster distribution. From the theory of thermodynamic fluctuations [69], the equilibrium cluster size distribution is given by

$$
f_{e q}(n)=f_{e q}(1) \exp -\frac{\Delta G(n)}{k_{B} T},
$$


where the factor $\exp -\frac{\Delta G(n)}{k_{B} T}$ is related with the probability of obtaining a cluster of the new phase containing $n$ monomers, and $\Delta G(n)$ is the minimum reversible work of formation of an $n$-cluster. Therefore, the problem is formulated now in terms of the free energy of droplet formation.

Classical nucleation theory, calculate this free energy by using the so-called capillarity approximation. In this approximation, clusters are assumed to be spherical and to have the same properties as the macroscopic bulk phase with a sharp interface. The free energy $\Delta G(n)$ can then be expressed, following Gibbs, as the sum of volume and interfacial energy contributions

$$
\Delta G(n)=-n \Delta \mu+\sigma A(n) .
$$

Here $\Delta \mu$ is the difference of chemical potentials per molecule between the gas and liquid phase, and $\sigma$ is the surface tension. For undercooled vapors, and assuming that the gas behaves as ideal, $\Delta \mu$ is given by

$$
\Delta \mu=k_{B} T \ln S,
$$

being $S=p / p_{e q}$ the supersaturation ratio or supersaturation. Using the previous expression and taking into account the spherical shape of the cluster, the free energy $\Delta G(n)$ can alternatively be written as

$$
\Delta G(n)=-n k_{B} T \ln S+\sigma s_{1} n^{2 / 3} .
$$

The critical size $n^{*}$ is defined as the size at which the free energy has a maximum

$$
\left.\frac{\partial \Delta G(n)}{\partial n}\right|_{n^{*}}=0
$$

Clusters smaller than the critical size tend to shrink, while those larger than $n^{*}$ can be considered as nuclei of the new phase as they will on average grow. For the case of spherical clusters, the critical size is

$$
n^{*}=\left(\frac{2}{3} \frac{\theta}{\ln S}\right)^{3}
$$

where

$$
\theta=\frac{\sigma s_{1}}{k_{B} T}
$$

is the non-dimensional surface tension. That correspond to a maximum value of the free energy 


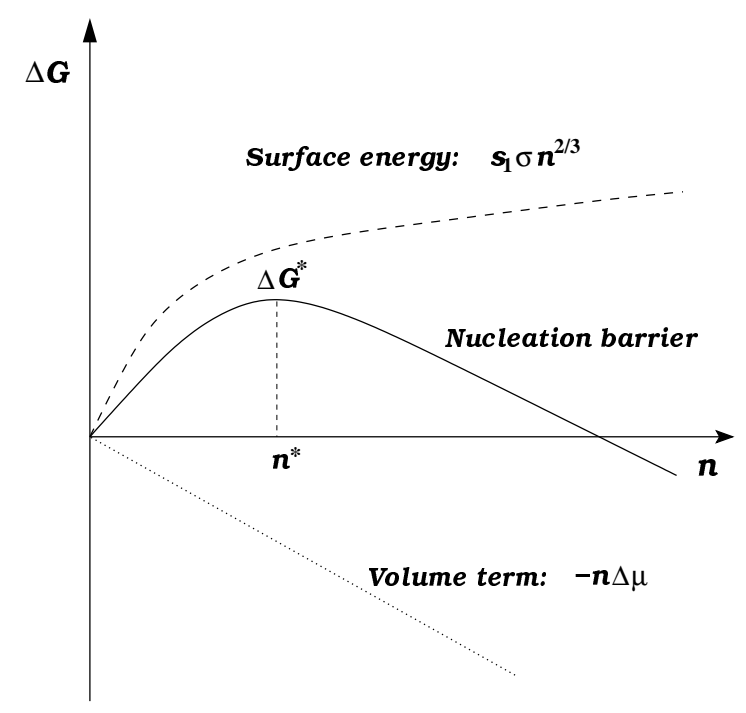

Figure 1.7: Free energy of formation of a liquid droplet in the classical nucleation picture, and its surface and bulk contributions.

$$
\Delta G_{C N T}^{*}=\frac{4}{27} \frac{\theta^{3}}{\ln ^{2} S} k_{B} T .
$$

This maximum is referred to as the nucleation barrier, and the rate $J\left(n^{*}, t\right)$ at which critical-sized embryos are formed is the nucleation rate.

\section{Classical Nucleation Theory of Crystallization}

The analysis presented in the previous section was introduced to describe nucleation of drops from a vapor. However, it is also the basis of crystallization of liquid substances.

In the classical approach to crystallization, capillarity approximation is also employed, and the free energy of formation of a crystal of $n$-molecules is expressed again as the sum of volume and interfacial energy contributions

$$
\Delta G(n)=v(n) \Delta g+\sum \sigma_{i} A_{i}
$$

where the sum accounts for the different surfaces that the crystalline structure may present. In the previous expression, $v(n)$ is the volume of the cluster, $\Delta g$ is the change of free energy per unit volume, and $\sigma_{i}$ is the interface tension of the surface $i$ with area $A_{i}$. The degree of metastability of an undercooled liquid 


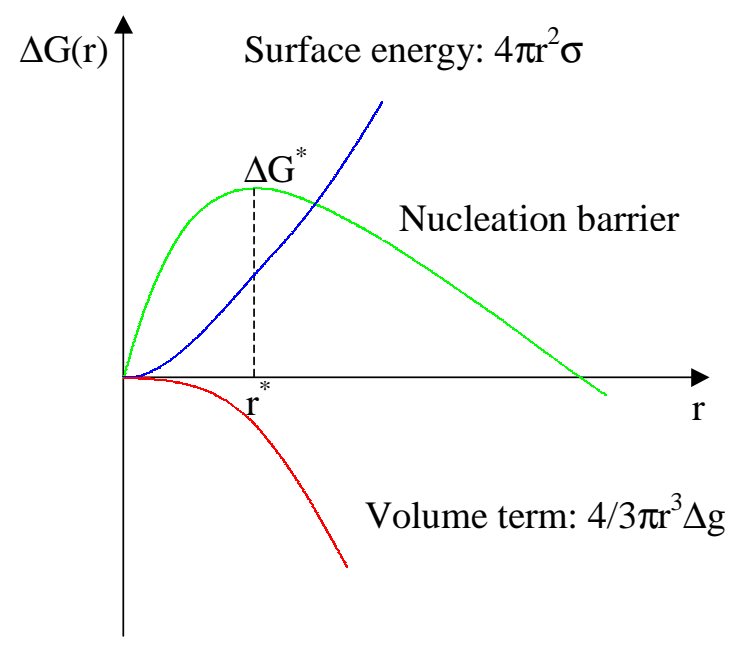

Figure 1.8: Free energy of formation of a crystal embryo in the classical nucleation picture, and its surface and bulk contributions.

is characterized by the undercooling $\Delta T \equiv T-T_{m}$, where $T_{m}$ is the equilibrium melting temperature and $T$ is the actual temperature. This undercooling is equivalent to the supersaturation in condensation, and the gain in free energy $\Delta g$ in the phase transition depends on it. In fact, neglecting the temperature dependence of the heat of fusion $\Delta h$, as a first approximation $\Delta g$ is given by

$$
\Delta g \simeq \frac{\Delta h \Delta T}{T_{m}}
$$

Consequently, $\Delta g$ is positive above $T_{m}$ and it is negative below that temperature.

For the simplest case of spherical crystal clusters, the crystallization free energy splits up into bulk and surface contributions (see Fig. 1.8) and is given by

$$
\Delta G=\frac{4 \pi r^{3}}{3} \Delta g+4 \pi r^{2} \sigma,
$$

with $r$ being the radius of the spherical crystal. This equation can alternatively be written in terms of the number of monomers as

$$
\Delta G=a n+b n^{2 / 3} \sigma
$$


where $a=\Delta g \bar{v}$ is the free energy per monomer and $b=(36 \pi)^{1 / 3} \bar{v}^{2 / 3}$, with $\bar{v}$ the molecular volume.

For the case of spherical clusters, the critical size and the critical radius are

$$
n^{*}=\frac{32 \pi}{3 \bar{v}} \frac{\sigma^{3}}{|\Delta g|^{3}}
$$

and

$$
r^{*}=\frac{2 \sigma}{|\Delta g|}
$$

which correspond to the maximum value of the free energy

$$
\Delta G^{*}=\frac{16 \pi}{3} \frac{\sigma^{3}}{\Delta g^{2}}
$$

However, the extension of the classical theory to crystallization presents some additional difficulties. The first one concerns the absence of independent measurements of the solid-liquid surface free energy $\sigma$ in the undercooled regime. In fact, the surface free energy is mostly evaluated precisely by fitting the results of nucleation rate experiments to the classical expression for the rate.

The second difficulty is related to the lack of a reliable kinetic approach to estimate the forward rate $k^{+}(n)$. The most usual approach, due to Turnbull and Fisher [70], proposed the following expression for this rate

$$
k^{+}(n)=4 n^{2 / 3} \Gamma \exp \left(-\frac{\delta G}{2 k_{B} T}\right) .
$$

Here $\delta G=\Delta G(n+1)-\Delta G(n)$, the factor $4 n^{2 / 3}$ arises from consideration of the number of available attachment sites on the surface of a spherical cluster and $\Gamma$ is the unbiased molecular jump frequency at the cluster interface, that can alternatively be expressed as

$$
\Gamma=\frac{k_{B} T}{h} \exp \left(-\frac{\epsilon}{k_{B} T}\right),
$$

where $\epsilon$ is the activation energy defined as the difference between the energy of the activated state and the average energies of the initial and final states, and $h$ is the Planck constant. This jump frequency $\Gamma$ is often identified with the jump frequency for bulk diffusion, through the atomic jump distance $\lambda$ and the spatial diffusion coefficient $D$, as

$$
\Gamma=\frac{6 D}{\lambda^{2}}
$$


Consequently, in this model the attachment rate $k^{+}(n)$ is proportional to the bulk diffusion coefficient $D$. As we will illustrate in Chapter 5 , the presence of flows, thermal gradients or hydrodynamic interactions may alter significantly this diffusion coefficient and thus the kinetic of the nucleation process.

An additional problem refers to the difficulties in identifying the nucleus in the case of crystallization from the melt. In that sense, promising approaches defining the cluster in terms of local order, which differs between the liquid and the crystal, are being developed [71].

All these difficulties imply that, at the present state, one cannot perform direct quantitative tests of nucleation rate experiments against crystal nucleation theories. For that reason, in this and in the remaining chapters, we will focus on nucleation from the vapor-phase. Nevertheless, qualitative discussions about the relevance of nonequilibrium effects on crystallization will be provided, and a simplified analysis of crystallization at advanced stages will be introduced in Chapter 6.

\section{Steady-State Nucleation Rate}

Classical nucleation theory assumes that a steady state, in which the populations of different cluster sizes no longer depend on time, is rapidly established. In this situation, following Eq. (1.2), the flux has the same value irrespective of the size, i.e. $J(n, t)=J$. This stationary value of the flux is often referred to as the nucleation rate, and by recurrence from equation (1.3) it is given by

$$
J=N_{t o t}\left[\sum_{n_{1}}^{n_{2}} \frac{1}{k^{+}(n) f_{e q}(n)}\right]^{-1},
$$

where $N_{\text {tot }}$ is the total number density of clusters, and cluster sizes $n_{1}$ and $n_{2}$ are such that for $n \leq n_{1}, f(n)=f_{e q}(n)$ and for $n>n_{2}, f(n)=0$. Consequently, the value $n_{1}$ represents the smaller cluster distinguishable from equilibrium fluctuations in the metastable phase, and $n_{2}$ is a postcritical large enough stable cluster. It has been shown that the nucleation rate does not strongly depend on the values of the boundary sizes $n_{1}$ and $n_{2}$ [72].

Replacing the summation in equation (1.25) by an integral, introducing the "equilibrium" distribution $f_{e q}(n)$, and evaluating the resulting integral by the steepest descent method (see Appendix), one obtains the classical expression for the nucleation rate

$$
J_{C N T}=k^{+}\left(n^{*}\right) Z N_{t o t} \exp \left[-\frac{\Delta G^{*}}{k_{B} T}\right]
$$


where

$$
Z=\sqrt{\frac{-\left.\frac{\partial^{2} \Delta G}{\partial n^{2}}\right|_{n *}}{2 \pi k_{B} T}}
$$

is frequently referred to as the Zeldovich factor [73].

This result is obtained under the assumption that the steady-state is instantaneously achieved which implies that nucleation proceeds at a constant rate. While frequently true, this assumption becomes wrong in some specific cases. A finite time is required in order for the concentration of clusters and the fluxes to attain their stationary values. This time lag can be of the same order than the measure time, specially in the case of polymers and glasses, and transient kinetics may thus be important in the description of crystallization. We shall not discuss in detail this subject here. Instead, we refer the interested reader to the works of Shi [74], Shneidman [75], and the references quoted in [15].

To describe the time-dependent nucleation, it is convenient to introduce a continuous version of the master equation (1.2).

\section{Continuous Approaches to the Master Equation}

As we have shown previously, in the classical nucleation theory the kinetics of the nucleation process is described through the master equation (1.1) for the evolution of the population of clusters. However, owing to the discrete nature of the growing process, resulting essentially from the progressive addition of atoms to the emerging cluster, the master equation provides a hierarchy of kinetic equations for the evolution of clusters of different sizes which is very difficult to solve. That is the main reason to abandon the master equation approach and to formulate a continuous diffusion equation to describe nucleation processes. The diffusion equation corresponding to the master equation (1.1) adopts the general form

$$
\frac{\partial f}{\partial t}=\frac{\partial}{\partial n}\left\{D_{n}(n) \frac{\partial f}{\partial n}+A(n) f\right\},
$$

where $A(n)$ and $D_{n}(n)$ are the drift and diffusion coefficients, respectively, which are related to the attachment rate $k^{+}(n)$ and the energy barrier $\Delta G(n)$.

The proper choice of these coefficients constitutes a controversial issue to the extent that several different expressions have been derived and proposed in the literature. Most of them result from continuous approaches to the master equation (1.1). However, the resulting equation is not unique. 
The most straightforward approach consists of replacing the discrete variable $n$ by a continuous variable $x$. After expanding Eq. (1.1) in a Taylor series we arrive at the well-known Kramers-Moyal [76]-[77] expansion, which upon truncation in second order yields the desired Fokker-Planck equation. This equation, however, has the serious inconvenience that it does not reproduce the equilibrium distribution of the original master equation. Additionally, it has also been proven that it overestimates the nucleation rate exponentially, with an error that grows unboundedly for large $n$ [78].

The Fokker-Planck equations of Frenkel-Zeldovich [79], Goodrich [80] and Shizgal-Barret [81] try to solve these difficulties by imposing a relationship between drift and diffusion coefficients in order to ensure the correct equilibrium distribution. These three proposals differ in the expressions for those coefficients, chosen to improve the approximation. However, as pointed out by Gitterman and Weiss [82], none of them seem to be able to reproduce the correct deterministic behavior.

A variant of these equations is the one formulated by Rabin and Gitterman [83], where the innovation consist in considering that the coefficients of the Fokker-Planck equation cannot be fixed arbitrarily, but rather - in the vicinities of the critical point - they are determined by the critical dynamics of the system.

Van Kampen [84] proposed an alternative method, consisting in rescaling the rate constants $k^{+}(n)$ and $k^{-}(n)$ in terms of a parameter $\Omega$ (usually the system size) and to expand the result in powers of $\Omega^{-1}$. In this way one obtains an expansion similar to Kramers-Moyal that shares the same problems described previously.

The contribution of Grabert, Hänggi and Oppenheim [85] and later Hänggi et al. [86] is also based on a rescaling of the rate constants, but they obtain the coefficients (expressed in terms of the rates $k^{+}(n)$ and $k^{-}(n)$ ) from nonlinear transport theory. Their equation reproduces the equilibrium distribution appropriately, but it does not describe the short time behavior correctly and it gives an erroneous value of the variance of the distribution [82].

We can also quote the essentially mathematical method of $\mathrm{Wu}[12],[87]$ that proposes a discrete mathematical optimization to minimize the error associated to approach the master equation - discrete - by means of a - continuous - diffusion type expression.

Finally, a more physical approach has been proposed by Shi [88]. Using a mesoscopic kinetic description of inhomogeneous nonequilibrium processes, his results support the validity of the Frenkel-Zeldovich expression, with the particularity that the rate constant should be evaluated at $n+1 / 2$ instead of at $n$. Quantitatively, however, this correction is not very significant and is usually 
ignored.

In spite of the conceptual difficulties and the previous controversy, the most widely used equation is the classical Frenkel-Zeldovich expression

$$
\frac{\partial f(n, t)}{\partial t}=\frac{\partial}{\partial n}\left(k^{+}(n) f_{e q}(n) \frac{\partial}{\partial n} \frac{f(n)}{f_{e q}(n)}\right)
$$

which is the basis of the calculation of nucleation rates.

\subsubsection{Phenomenological Modifications to the CNT}

The limitations of the CNT revealed by the experiments have been the origin of an intense search for improved theoretical models able to describe nucleation. Phenomenological theories have pursued an improved expression for the work of formation of a cluster, without introducing any modification in the kinetics, and keeping the essentials of the capillarity approximation.

The macroscopic and equilibrium character of the capillarity approximation sustaining the CNT, involve some difficulties when one tries to extend its validity to the scope of a tiny nonequilibrium entity as a nucleating droplet. For instance, capillarity approximation does not include the translational, rotational, vibrational or configurational contributions to the free energy of a cluster. These statistical mechanical contributions are negligible in the thermodynamic limit, but may become relevant at the mesoscopic scale. The inconsistency problems of the CNT also arise at this mesoscopic level. In addition, the capillarity approximation does not take into account the dependency of the surface tension on the curvature. Many approaches have been proposed to overcome these and other difficulties. They are summarized in the following subsections.

\section{The Translational-Rotational Paradox}

One of the corrections to the CNT subject to an intense debate in last years originates from the proper accounting of the translational and rotational degrees of freedom of a small cluster. The work of Lothe and Pound [89] initiated an intense interest on what has been called the "translational-rotational paradox". The paradoxical aspect of the topic was that the apparently reasonable incorporation of the translational and rotational degrees of freedom lead to a significant correction to the free energy of formation of a cluster, which resulted in modifications of nucleation rates by factors on the order of $10^{17}$, thus destroying any reasonable agreement of the CNT with experiments. The paradox has been solved by the recent work of Reiss, Kegel and Katz [90], after a long-standing 
controversy. We shall discuss in more detail this subject and the proper role played by translational and rotational degrees of freedom in Chapter 4 .

\section{Self-Consistency Corrections}

The classical expression for the work of formation of a $n$-cluster presents the serious inconsistency that it predicts a non-zero value for the energy required to create a $n=1$ cluster, i.e. a monomer, $\Delta G(1)=-k_{B} T \ln S+\sigma s_{1} \neq 0$. Since the Gibbs free energy for nucleation is defined relative to the monomer state, it should accordingly become zero at $n=1$. To restore this consistency requirement, several corrections have been proposed in the literature (see for instance Refs. [91],[92] and the careful analysis of Ref. [12]). The most popular proposal consists of simply subtracting $\Delta G(1)$ to $\Delta G$, yielding a modification factor to the nucleation rate of $e^{\theta} / S$. This term increases nucleation rates by a factor of $10^{6}-10^{8}$, deteriorating in general the agreement with experiments, but tends to offset the erroneous trend of the classical theory of predict too high nucleation rates at high $T$, and too low ones at low $T$. Whereas the factor $1 / S$ is commonly accepted, the other is not, and also the requisite of consistency is still discussed by some authors. The work of Reiss, Kegel and Katz [90] also seems to have shed some light on this topic.

\section{Theories Based on the Fisher's Droplet Model}

A general phenomenological expression for the free energy of formation of a cluster was proposed by Fisher in the form [93],[94]

$$
\frac{\Delta G(n)}{k_{B} T}=-n \ln S+\kappa_{n} \theta n^{\gamma}+\tau \ln n+\ln \left(V q_{0}\right)
$$

In this expression, the first and second terms are the bulk and surface contributions, respectively, the two contributions considered in CNT. The parameter $\kappa_{n}$ is a function of the size and the temperature which describes deviations of the surface free energy from that of a macroscopic cluster; and $\gamma$ is a parameter allowing different cluster shapes $(\gamma=2 / 3$ for a spherical cluster). The remaining terms include rotational, translational, vibrational, configurational, and entropic contributions related to additional degrees of freedom which capillarity approximation may leave out. In those terms, $V$ is the volume, and $\tau$ and $q_{0}$ are parameters whose expressions and values are different for each theory.

Several phenomenological theories have been proposed in which the expression of the free energy resembles the one formulated through Eq. (1.30). 
Based on the Fisher's model for the cluster, Dillmann and Meier [95],[96] introduced a theory where the $\kappa_{n}$ factor was aimed to correct the curvature dependence of the surface tension. They proposed a functional form for this factor in terms of an expansion in powers of the inverse of the radius, inspired on the work of Tolman [97]

$$
\kappa_{n}=1+\alpha_{1} n^{-1 / 3}+\alpha_{2} n^{-2 / 3} .
$$

The values of the parameters $\alpha_{1}, \alpha_{2}, \tau$ and $q_{0}$ were fitted to reproduce the critical and low density properties of each substance. Unexpectedly, $\tau$ and $q_{0}$ turned out to be almost the same for all substances and the predictions of the Dillmann and Meier theory agree surprisingly well with the experimental data for alcohols [96].

Ford et al. [98],[99] recently discovered a serious inconsistency in the original Dillmann and Meier theory (they considered an ideal gas but fitted the results to imperfect vapor properties) and proposed a correction to reestablish its validity. Unfortunately, the corrections alter the predictions of the model considerably and completely spoil the good agreement with the experimental data. In an attempt to preserve the original accordance with experiments of the Dillmann and Meier theory, but overcoming its flaws, Delale an Meier [100] introduced the exponent $\gamma$ of the surface term in Eq. (1.30) as another fitting parameter. Its value was adjusted to fit the critical properties for each substance. The results were quite correct, but the theory has too many fitting parameters.

Finally, Kalikmanov and van Dongen [101] proposed a similar theory but only using the first term in the expansion of Eq. (1.31), obtaining good accordance with experimental data for some alcohols.

\section{Scaled Nucleation Theories}

The scaling of the nucleation rate with the temperature in the vicinities of the critical point had been extensively considered in the literature. McGraw [18] demonstrated that experimental data for critical supersaturations also follow scaling laws for temperatures far below the critical temperature $T_{c}$. Motivated by this work and based on the CNT, Hale [102] proposed a scaled theory for the nucleation rate that suggested a universal dependence of critical supersaturations on $\left(T_{c} / T-1\right)$ for $T \ll T_{c}$

$$
\ln S_{c r i t} \sim 0.53 \frac{\left(T_{c} / T-1\right)^{3 / 2}}{\Omega^{3 / 2}}
$$


where $\Omega$ is a material-dependent constant related to the excess surface entropy per molecule. The model seems to work reasonable well in some cases where CNT fails.

Recently, McGraw and Laaksonen [103] proposed scaling relations for the number $n^{*}$ of molecules in the critical nucleus and the nucleation barrier $\Delta G^{*}$. Based on the CNT and the validity of the nucleation theorem (analyzed in detail in Chapter 3), they proposed two scaling theorems

$$
\begin{gathered}
n^{*}=C(T) \Delta \mu^{-3}, \\
\Delta G^{*}-\Delta G_{C N T}^{*}=-D(T),
\end{gathered}
$$

whose validity was confirmed for some cases by density functional calculations [104], being $C(T)$ and $D(T)$ scaling parameters functions only of $T$. These scaling relations seem to provide a simple explanation and parametrization of the systematic temperature-dependent departure of CNT predictions and experiments for some substances.

\section{Diffuse Interface Theory}

Gránásy and collaborators [105],[106] have recently introduced a particular approach to calculate the work of formation of a droplet which goes beyond the capillarity approximation by using a diffuse interface model. This model assumes a parametrization of the enthalpy and entropy density profiles of droplets having the bulk physical properties at the center and a "diffuse interface" whose thickness is size independent. The rates predicted by this model have been shown to be consistent with the experimental data of some non-polar substances and crystals.

\section{Adiabatic Nucleation Theory}

A very recent phenomenological approach [107] even question the isothermal nature of the nucleation process, which is a key assumption in the CNT, and proposes that nucleation proceeds adiabatically. Although some thermodynamic relations have been derived, the theory is still not able to predict actual nucleation rates.

\subsubsection{Kinetic Models}

Unlike phenomenological theories, kinetic models of nucleation are focused on the direct calculation of the evaporation and the condensation rate coefficients 
without invoking the constrained equilibrium hypothesis or resort to free energies of cluster formation. Some of these kinetic approaches are based on the peculiar dynamics of a monomer near the surface of a cluster, which is described by means of Fokker-Planck equations. Within this approach we will consider the works of Lovett [108], and Ruckenstein and collaborators [109]-[112].

Lovett [108] uses Thermodynamics to estimate the radius of the critical cluster and the height of the energy barrier that a monomer should surmount to leave the cluster surface. Considering that the monomer undergoes a Brownian motion, he uses the corresponding Fokker-Planck equation to study the probability of leaving the cluster. His model is only applicable to nucleation in gases, and he uses the macroscopic concept of interfacial tension which can doubtfully be applied to the molecular description of small clusters.

Ruckenstein and coworkers [109]-[112] propose an alternative approach based on the direct calculation of the evaporation rate $k^{-}$. Their starting point is the estimation of the potential barrier that is generated at the surface of a spherical cluster of radius $R$ from the interaction between monomers and the recount of the number of monomers that interact with a given one. They also consider that the molecules undergo Brownian motion. In the case of nucleation in liquids, they write a Smoluchowski equation in spherical coordinates for the distribution of probability $\rho(\vec{r}, t)$

$$
\frac{\partial \rho(\vec{r}, t)}{\partial t}=\vec{\nabla}_{\vec{r}} \cdot\left(D \vec{\nabla}_{\vec{r}} \rho(\vec{r}, t)+\frac{D}{k T} \rho(\vec{r}, t) \vec{\nabla}_{\vec{r}} \phi(\vec{r})\right) .
$$

Here $D$ is the diffusion coefficient and $\phi(\vec{r})$ the interaction potential of a surface monomer. For gases, they use a diffusion equation in the energy space. These equations are just employed to obtain the rates $k^{+}$and $k^{-}$, as the mean first passage time of a monomer crossing the potential barrier generated at the surface of the cluster. The rates are introduced in the common Kramers-Moyal truncated expansion of the master equation to evaluate the nucleation rate.

The main inconveniences of this formalism are that it considers (in the case of liquids) that the diffusion coefficient is constant, when in fact there are reasons to think that it is not certainly so; moreover the contribution of the bulk medium to the effective potential is not incorporated rigorously [14]. On the other hand, an important difficulty linked to this type of descriptions arises from the fact that the nucleation rate is very sensitive to small uncertainties in the interatomic potential [113].

The kinetic approach of Bauer et al. [114]-[116] extends the usual kinetic model for addition and loss of molecules to a cluster by accounting for the interaction of the clusters with the background carrier gas. Therefore, the theory 
requires double the usual number of kinetic coefficients which are adjusted, after many assumptions, to fit the experimental data.

Finally, another very recent approach to analyze vapor-phase nucleation is the Dynamic Nucleation Theory [117], which proposes a method to evaluate the rate constants for cluster evaporation and condensation by means of the variational transition state theory. It could be considered as an hybrid treatment between kinetic and molecular theories.

\subsubsection{Field and Density Functional Approaches}

One of the main drawbacks of the classical theory is the assumption that the interface of the cluster is sharp, hypothesis that -at least at the level of simulationsis not completely satisfactory.

Density functional models allow a treatment keeping in mind the possibility that the interface between the cluster and the original phase may be diffuse. The basic assumption is the consideration of a nucleation event as an inhomogeneous fluid of average density $\rho(\mathbf{r})$. Then, it can be proved [118] that there exists a free-energy functional of the density $\Omega[\rho(\mathbf{r})]$ whose minima determine the thermodynamic stable states at a given temperature. In the particular case of nucleation, the evaluation of the saddle-point $\delta \Omega / \delta \rho(\mathbf{r})=0$ yields the density profile of the critical nucleus, and from it the nucleation barrier $\Delta \Omega^{*}$ can be calculated. Since density functional approaches provide no information about kinetic factors, the nucleation rate must be evaluated from the classical steadystate formula Eq. (1.26).

The first density functional for nucleation was proposed by Cahn and Hilliard [119],[120]

$$
\Omega[\rho(\mathbf{r})]=\int d \mathbf{r}\left\{f_{h}[\rho(\mathbf{r})]-\mu \rho(\mathbf{r})+K[\nabla \rho(\mathbf{r})]^{2}\right\},
$$

where $f_{h}$ is the Helmholtz free energy per unit volume of the homogeneous system with density $\rho$, and the square gradient-term $K[\nabla \rho(\mathbf{r})]^{2}$ accounts for non-local contributions to the free energy, required to incorporate the energetic cost associated with the spatial variation of the density.

For very sharp interfaces, the square-gradient approximation is not sufficiently good. To avoid this difficulty, Oxtoby and coworkers [121]-[123] have proposed a density functional constructed directly from the interaction potential between molecules

$$
\Omega[\rho(\mathbf{r})]=\int d \mathbf{r}\left\{f_{h}[\rho(\mathbf{r})]-\mu \rho(\mathbf{r})\right\}+\iint d \mathbf{r} d \mathbf{r}^{\prime} \phi_{a t t}\left(\left|\mathbf{r}-\mathbf{r}^{\prime}\right|\right) \rho(\mathbf{r}) \rho\left(\mathbf{r}^{\prime}\right),
$$


where the first integral accounts for the hard-sphere, purely repulsive interaction, and $\phi_{a t t}$ represents the long range attractive part of the potential. Interesting investigations have been performed on different systems and using different potentials [124]-[126]. The comparison with macroscopic phenomenological theories results in significant discrepancies. The formalism has also been extended to describe crystallization [127]-[128].

The main inconvenience of these conventional density functional theories are that their correctness strongly depends on the appropriate election of the free energy functional. Moreover, its range of application is restricted to steady-state situations.

The contributions described above are exclusively centered on the evaluation of the nucleation barrier and do not deal with the real kinetics of the process. An interesting approach to incorporate kinetic aspects into density functional theories for nucleation was developed by Langer [129]-[131]. He proposed a first-principle calculation of the rate of decay of a metastable state, based on Statistical Mechanics and including fluctuations. Starting from the Hamiltonian dynamics of the system, he was able to derive the following equation

$$
\frac{\partial \rho(\{\eta\}, t)}{\partial t}=\sum_{i, j} \frac{\partial}{\partial \eta_{i}}\left(M_{i j}\left(\frac{\partial \rho}{\partial \eta_{j}}+\frac{\partial G(\{\eta\})}{\partial \eta_{j}} \frac{\rho}{k T}\right)\right),
$$

where $\{\eta\}$ is the set of all degrees of freedom (coordinates and momenta) that characterize the system configuration, $G(\{\eta\})$ is the coarse-grained free energy functional, and $M_{i j}$ is a generalized mobility matrix. From this Fokker-Planck equation, the nucleation rate is given by the flow across the saddle point of $G(\{\eta\})$, which includes a pre-exponential statistical factor (a multi-dimensional generalization of the Zeldovich factor) and a kinetic factor (related to the deterministic growth rate).

Langer and Turski [132] applied this approach to construct a hydrodynamic model to describe the condensation of a supersaturated vapor. The length-scale requirements of the hydrodynamic description restrict the validity of the model to the vicinities of the critical point. The results obtained were nearly the same as the classical ones.

\subsubsection{Molecular Theories}

The aim of molecular approaches is to obtain nucleation rates starting directly from the potential energy of interaction between molecules. The main procedure relays on the use of statistical mechanics, instead of macroscopic phenomenological models, to evaluate the equilibrium distribution of clusters. These theories 
have a strong connection with simulations, since simulations often provide the only tool able to evaluate the equilibrium distributions proposed by them. A very comprehensive and complete revision of molecular theories, including a historical perspective and connections with other theories, is given in Ref. [133].

The centerpiece underlying the development of any molecular theory is to know what is the relevant and physically meaningful definition of a cluster. In this context, many proposals can be found in the literature.

One possible way to identify a cluster, introduced by Hill [134], is as the group of molecules whose total energy is negative. However, this criterion has the serious inconvenient of being very difficult to implement in practice.

Another possible and more wieldy definition of cluster was proposed by Stillinger [135]. He defined the cluster in terms of a connectivity criterion. Two molecules are connected if their centers are separated by a distance shorter than some given maximum $r_{c}$. The cluster is then defined by the group of molecules which lie within a distance $r_{c}$ of at least one other molecule. This definition of cluster is very useful for simulations, but presents some redundancy problems as pointed out in Ref. [136].

The LBA cluster, introduced by Lee, Barker and Abraham [137], consist of $n$ molecules confined in a rigid spherical container of volume $v$, whose center coincides with the center of mass of the association of molecules. For intermediate sizes, the cluster is stable and its free energy is independent of the volume $v$, but there is no natural criterion to justify the volume $v$ of the constraining cell.

A significant effort toward a true molecular theory of nucleation has recently been made by Reiss and collaborators [136],[138]-[143]. Trying to avoid the problem of the arbitrary choice of the constraining volume in the LBA cluster, they introduced the concept of $n / v$ cluster [143], in which the volume $v$ also plays the role of an independent variable. The criteria to define the volume is that the spherical shell $d v$ centered on the center of mass of the molecules, should contain the "last" molecule. All possible configurations of the molecules preserving the position of the center of mass and a molecule in the shell constitute, collectively, the $n / v$ cluster.

Finally, the most satisfactory definition of clusters seem to be the recently proposed $n / v$-Stillinger cluster [133], an hybrid that combines the Stillinger connectivity criterion with the $n / v$ cluster. The cluster consist of all possible configuration of $n$ molecules within $v$ that satisfy the connectivity condition, conserve the position of the center of mass, and maintain the shell molecule within the shell. This seems to be a quite consistent definition of cluster, allows a reasonable simulative estimation of free energies, and may provide the basis 
of future developments of phenomenological and molecular theories.

\subsection{Simulations}

The other ingredient which has contributed to the renewed interest in nucleation is the development of computer simulations. Nowadays, computer simulations offer the possibility of exploring the process at molecular scale then witnessing the real formation and evolution of clusters. In addition, they are not subjected to some of the limitations and inconveniences inherent to the phenomenological descriptions.

There exist two primary problems that simulations must cope with. The first one is that nucleation is a rear event and thus difficult in nature to simulate. Typical values of the parameters that can be handled in simulations (such as volume, time and number of particles) restrict the scope of direct simulations to experimentally unreachable conditions or isolated (artificial) clusters. The second problematic issue of simulations is related with the troubles of definition and identification of clusters. It is not clear what is the proper prescription which has to be employed to identify which particles belong to the same cluster at a given time, as was discussed in the context of molecular theories.

According to the objective of the simulations, we can roughly distinguish between two different branches: simulations aimed to estimate the nucleation barriers, and the ones allowing the direct estimation of nucleation rates.

\subsubsection{Evaluation of Nucleation Barriers}

One of the principal applications of computer simulations is the evaluation of the energy of droplet formation directly from the interactions between molecules. The evaluation of energy barriers can be performed either by Monte Carlo or by molecular dynamics techniques.

Monte Carlo simulations [137],[144]-[152] provide the more straightforward technique to evaluate equilibrium thermodynamic properties of a droplet. In particular, its energy of formation can be calculated through the exploration of equilibrium configurations of the molecules, since the equilibrium probability of appearance of a cluster containing $n$ molecules, at a given temperature and supersaturation, is related to the energy involved in its formation through Eq. (1.7). The problem is that the appearance of a cluster under experimental conditions is an extremely rare event in simulations. Therefore, using the standard Boltzmann's sampling of configurations is almost impossible to observe a critical droplet in the duration of a simulation. Consequently, the direct estimation of 
nucleation barriers is restricted to somewhat unrealistic conditions, unless one uses a different sampling of the configurational space.

One of these non-standard techniques is the umbrella-sampling [146], applied to nucleation by Frenkel and collaborators [147]-[149], that permits the estimation of nucleation barriers at low and experimentally attainable supersaturations.

Another trick to avoid the computational restrictions of a large scale simulation is to perform a small-system simulation of a single, constrained cluster. For instance, Kusaka et al. [153],[154] have proposed an efficient method to identify relevant clusters by a grand-canonical Monte Carlo simulation in a small cell whose results are rescaled to the system size. This mapping of small-system fluctuations into macrostates has been critically reconsidered in Refs. [155]-[157]. A different small-system grand-canonical Monte Carlo simulation, following the physical cluster theory of Reiss and coworkers, has recently been implemented by Oh and Zeng [158].

Molecular dynamics (MD) simulations can also be applied to estimate free energies of droplet formation [159]-[161]. The molecular dynamics technique follows the time evolution of a microcanonical ensemble of particles interacting via a particular intermolecular potential. The integration of the Newton's equations for all particles provides the real trajectory of the system in the phase space from which its properties can be evaluated. However, the use of this method to calculate equilibrium properties is in general less efficient than the Monte Carlo approach, because it requires long equilibration times. Where MD simulations have become really valuable is in the direct evaluation of nucleation rates.

\subsubsection{Evaluation of Nucleation Rates}

Molecular dynamics simulations offer the interesting possibility of monitoring the time evolution of the nucleation process and hence estimate directly nucleation rates. Unfortunately, even with the present-day powerful computers, it is almost impossible to directly simulate the average rate of nucleus formation, even on the simplest systems.

The "brute-force" application of the molecular dynamics technique would consist of simply undercooling the system and then waiting the appearance of nuclei. Since the nucleation barrier decreases as $1 /(\ln S)^{2}$, this type of simulations based on the recount of spontaneous formation of nuclei require experimentally non-affordable high supersaturations [162]. For more moderate and

realistic conditions the nucleation barrier is so large that a spontaneous crossing is extremely unlikely during the typical times of a simulation. 
Recently, a different type of simulations allowing the study of nucleation under more realistic undercoolings, has been introduced by ten Wolde, RuizMontero and Frenkel [163]-[165] in the context of crystallization. Their method is based on the separation of the simulation in two parts. First, either Monte Carlo or molecular dynamics simulations are employed to evaluate the nucleation barrier in terms of a reaction coordinate. This reaction coordinate constitutes in fact an order parameter connecting the initial and final phases and being sensitive to the global degree of local order (or crystallinity) in the system. Molecular dynamics is then used to simulate the evolution of the transition state. The Bennett-Chandler scheme [166]-[168], based on linear response theory and described in the next chapter, is employed to relate the decay rate of a fluctuation having the critical size, with the barrier-crossing rate. The combination of the probability that the system is located at the transition state, together with the barrier-crossing rate yield a prediction of the nucleation rate.

We also want to mention that, although in the different context of crystallization of colloidal particles, Auer and Frenkel [2] have very recently reported the first parameter-free estimate of a nucleation rate, demonstrating the power of computer simulations as a tool to investigate nucleation.

Lately, the interest has also been focused on the direct evaluation of the rate coefficients of condensation and evaporation, and a small-system MD simulation has been proposed [169].

\subsection{Conclusions}

At this point, it is important to stress out the main conclusions aroused by the previous revision on the subject.

Although an impressive progress has been accomplished in experimental techniques, the tremendously difficult nature of the measurement of nucleation rates provokes that substantial discrepancies between theory and experiments and even between experimental results, persist. These deviations reveal the real signification of experiments beyond the particular uncertainty estimations provided by the experimentalists. Consequently, the agreement with experimental results should not constitute the sole and absolute criterion by means one can judge the validity of any theory. In fact, and paradoxically, many approaches considered as more "consistent" than the classical theory yield worst results when tested against experiments.

Regarding the theory, in spite of its flaws, the Classical Nucleation Theory is still the reference in the field. Its success lies in the fact that it constitutes a simple theory with wide applicability which predicts nucleation rates in terms solely 
of easily accessible macroscopic properties. In addition, it has been successful in predicting the limits of metastability. However, its quantitative predictions for nucleation rates are not so correct. Besides, simulations have shown that the capillarity approximation in which CNT is based is not applicable to clusters of small size containing only a few molecules. In addition, the use of macroscopic properties for these mesoscopic droplets seems to be arguable.

Many extensions and phenomenological modifications of the CNT have been introduced to overcome these deficiencies, but these changes have not led to a general theory that is applicable and reliable for a variety of systems. Molecular theories have the incentive of providing strong realism of the process at microscopic scale, but the complexity of the definition of the relevant cluster limits somehow its applicability to simple intermolecular potentials. As with the phenomenological approaches, kinetic and molecular theories presented up to date have not resulted in a general theory routinely applied to calculate nucleation rates. That is the main reason why CNT is still widely used.

Finally, concerning simulations, its main contribution up to now has been in the characterization of nucleation clusters at molecular scale. At their present state they are still not able to provide a direct insight of nucleation rates. As a result, some indirect and very promising methods are being developed to evaluate nucleation rates [2]. The problem is that these methods do not resort to any of the nucleation theories described previously, so a big gap between simulations and theories is being opened.

In summary, although recent advances in the study of nucleation are very promising, much remains to be accomplished. In particular, the previous revision evidences that most of the efforts have been centered on the equilibrium aspects of the process -mainly on the calculation of the free energy of cluster formation- quite disregarding the nonequilibrium facet of the problem. Our objective will be to introduce a consistent framework to describe nucleation, retaining the two main features of this process, namely its mesoscopic and nonequilibrium character. This will be the topic of the following chapters. 


\section{Appendix}

\section{Evaluation of the Steady-State Nucleation Rate by Steepest De- scent}

The steady-state flux can be calculated from the stationary solution of the Fokker-Planck equation (1.29)

$$
0=\frac{\partial}{\partial n}\left(k^{+}(n) f_{e q}(n) \frac{\partial}{\partial n} \frac{f(n)}{f_{e q}(n)}\right)
$$

The boundary conditions are: $f / f_{e q}\left(n_{1}\right) \rightarrow 1$ as $n_{1} \rightarrow 1$ and $f / f_{e q}\left(n_{2}\right) \rightarrow 0$, as $n_{2} \rightarrow \infty$. In the steady state, the first integral of the equation above is

$$
k^{+}(n) f_{e q}(n) \frac{\partial}{\partial n} \frac{f(n)}{f_{e q}(n)}=J=\text { constant }
$$

where the constant $J$ is in fact the steady-state nucleation rate. The integration of this equation, using the boundary conditions described above, determines the expression for the steady-state nucleation rate

$$
J=\frac{1}{\int_{n 1}^{n 2} \frac{1}{k^{+}(n) f_{e q}(n)} d n}
$$

or alternatively

$$
J=\frac{1}{\int_{n 1}^{n 2} \frac{1}{k^{+}(n) f_{e q}(1)} \exp \left(\frac{\Delta G}{k_{B} T}\right) d n},
$$

where Eq. (1.7) has been used. When the nucleation barrier is very high, this integral can be evaluated by the steepest descent approximation. Notice that, due to the presence of the exponential in the integrand, the overwhelming contribution to the previous integral comes from a narrow region centered about the location of the maximum at $n^{*}$. We can then safely assume that $k^{+}(n) \cong$ $k^{+}\left(n^{*}\right)$, and replace the limits of integration $n_{1}$ and $n_{2}$ by 0 and $\infty$, respectively. In addition, the free energy barrier can be expanded around the critical size as follows

$$
\Delta G(n) \approx \Delta G\left(n^{*}\right)+\left.\frac{1}{2} \frac{d^{2} \Delta G(n)}{d n^{2}}\right|_{n^{*}}\left(n-n^{*}\right)^{2}
$$


The nucleation rate can then be written as

$$
J \approx k^{+}\left(n^{*}\right) f_{e q}(1) \exp \left(\frac{-\Delta G^{*}}{k_{B} T}\right)\left[\int_{0}^{\infty} \exp \frac{-\left.\frac{d^{2} \Delta G(n)}{d n^{2}}\right|_{n^{*}}\left(n-n^{*}\right)^{2}}{2 k_{B} T}\right]^{-1}
$$

The evaluation of the Gaussian integral yields

$$
Z=\sqrt{\left.\frac{-1}{2 \pi k_{B} T} \frac{d^{2} \Delta G(n)}{d n^{2}}\right|_{n^{*}}},
$$

which is frequently referred to as the Zeldovich factor. The final expression for the steady-state nucleation rate is then

$$
J=k^{+}\left(n^{*}\right) f_{e q}(1) Z \exp \left(\frac{-\Delta G^{*}}{k_{B} T}\right) .
$$




\section{Bibliography}

[1] D.B. Fahrenheit, Phil. Trans. Roy. Soc. 39, 78 (1724).

[2] S. Auer and D. Frenkel, Nature 409, 1020 (2001).

[3] U. Gasser, E.R. Weeks, A. Schofield, P.N. Pusey, and D.A. Weitz, Science 292, 258 (2001).

[4] M. Volmer and A. Weber, Z. Phys. Chem. 119, 277 (1926).

[5] L. Farkas, Z. Phys. Chem. 125, 236 (1927).

[6] R. Becker and W. Döring, Ann. Phys. 24, 719 (1935).

[7] M. Volmer, Kinetic der Phasenbildung (Theodor Steinkopff, Dresden, 1939), translated in English by Intelligent Department, AMC.

[8] J. Frenkel, J. Chem. Phys. 7, 538 (1939).

[9] J. Frenkel, Kinetic Theory of Liquids, (Dover, New York, 1955).

[10] D.W. Oxtoby, J. Phys. Condens. Matter 4, 7627 (1992).

[11] A. Laaksonen, V. Talanquer and D.W. Oxtoby, Annu. Rev. Phys. Chem. 46, 489 (1995).

[12] D.T. Wu, Solid State Phys. 50, 37 (1996).

[13] A.C. Zettlemoyer, Nucleation, (Marcel Dekker, New York, 1969).

[14] P.G. Debenedetti, Metastable Liquids: Concepts and Principles, Chap. 3, (Princeton University Press, 1996).

[15] K. F. Kelton, Solid State Phys. 45, 75 (1991).

[16] J.D. Gunton, J. Stat. Phys. 95, 903 (1999). 
[17] R.H. Heist and H. He, J. Phys. Chem. Ref. Data 23, 781 (1994).

[18] R. McGraw, J. Chem. Phys. 75, 5514 (1981).

[19] J.L. Katz and B.J. Ostermier, J. Chem. Phys. 47, 478 (1967).

[20] J.L. Katz, J. Chem. Phys. 52, 4733 (1970).

[21] R.H. Heist and H. Reiss, J. Chem. Phys. 59, 665 (1973).

[22] J.L. Katz, C.J. Scoppa II, N.G. Jumar, and P. Mirabel, J. Chem. Phys. 62, 448 (1975).

[23] J.L. Katz, P. Mirabel, C.J. Scoppa II, and T.L. Virkler, J. Chem. Phys. 65, $382(1976)$.

[24] P. Mirabel and J.L. Katz, J. Chem. Phys. 67, 1697 (1977).

[25] C. Flageollet-Daniel, P. Ehrhard, and P. Mirabel, J. Chem. Phys. 75, 4615 (1981).

[26] A. Kacker and R.H. Heist, J. Chem. Phys. 82, 2734 (1985).

[27] C-H. Hung, M.J. Krasnopoler, and J.L. Katz, J. Chem. Phys. 90, 1856 (1989).

[28] D. Kane and M.S. El-Shall, J. Chem. Phys. 105, 7617 (1996).

[29] M.M. Rudek, J.L. Katz, and H. Uchtmann, J. Chem. Phys. 110, 11505 (1999).

[30] M.M. Rudek, J.A. Fisk, V.M. Chakarov, and J.L. Katz, J. Chem. Phys. 110, 11505 (1999).

[31] M.M. Rudek, J.L. Katz, I.V. Vidensky, V. Zdímal, and J. Smolík, J. Chem. Phys. 111, 3623 (1999).

[32] R.H. Heist, M. Janjua, and J. Ahmed, J. Phys. Chem. 98, 4443 (1994).

[33] J.A. Fisk and J.L. Katz, J. Chem. Phys. 104, 8649 (1996).

[34] J.A. Fisk, V.M. Chakarov, and J.L. Katz, J. Chem. Phys. 104, 8657 (1996).

[35] A. Bertelsmann and R.H. Heist, J. Chem. Phys. 106, 610 (1997); and 106, 624 (1997) . 
[36] F.T. Ferguson and J.A. Nuth III, J. Chem. Phys. 111, 8013 (1999).

[37] D.Kane, S.P. Fisenko, M. Rusyniak, and M.S. El-Shall, J. Chem. Phys. 111, 8496 (1999).

[38] F.T. Ferguson, R.H. Heist, and J.A. Nuth III, J. Chem. Phys. 113, 7398 (2000).

[39] N. Schaeffer, F. Utheza, F. Garnier, and G. Lauriat, J. Chem. Phys. 113, 8085 (2000).

[40] M.P. Anisimov, S.D. Shandakov, Y.I. Polygalov, and R.H. Heist, J. Chem. Phys. 114, 899 (2001).

[41] M.P. Anisimov and A. Cherevko, J. Aerosol Sci. 16, 97 (1985).

[42] M.P. Anisimov, K. Hämeri, and M. Kulmala, J. Aerosol Sci. 25, 23 (1994).

[43] V. Vohra and R.H. Heist, J. Chem. Phys. 104, 382 (1996).

[44] K. Hämeri and M. Kulmala, J. Chem. Phys. 105, 7683 (1996); and 105, 7696 (1996).

[45] V.B. Mikheev, N.S. Laulainen, S.E. Barlow, M. Knott, and I.J. Ford, J. Chem. Phys. 113, 3704 (2000).

[46] H. Lihavainen, Y. Viisanen, and M. Kulmala, J. Chem. Phys. 114, 10031 (2001).

[47] J.L. Schmitt, Rev. Sci. Instrum. 52, 1749 (1981).

[48] J.L. Schmitt, G.W. Adams, and R.A. Zalabsky, J. Chem. Phys. 77, 2089 (1982).

[49] R.C. Miller, R.J. Anderson, J.L. Kasner Jr., and D.E. Hagen, J. Chem. Phys. 78, 3204 (1983).

[50] J.L. Schmitt, R.A. Zalabsky, and G.W. Adams, J. Chem. Phys. 79, 4496 (1983).

[51] G.W. Adams, J.L. Schmitt, and R.A. Zalabsky, J. Chem. Phys. 81, 5074 (1984).

[52] P.E. Wagner and R. Strey, J. Chem. Phys. 80, 5266 (1984). 
[53] R. Strey, P.E. Wagner, and T. Schmeling, J. Chem. Phys. 84, 2325 (1986).

[54] R. Strey, T. Schmeling, and P.E. Wagner, J. Chem. Phys. 85, 6192 (1986).

[55] Y. Viisanen, R. Strey, and H. Reiss, J. Chem. Phys. 99, 4680 (1993).

[56] Y. Viisanen and R. Strey, J. Chem. Phys. 101, 7835 (1994).

[57] J. Hruby, Y. Viisanen, and R. Strey, J. Chem. Phys. 104, 5181 (1996).

[58] M.P. Anisimov, P.K. Hopke, S.D. Shandakov, and I.I. Shvets, J. Chem. Phys. 113, 1971 (2000).

[59] A. Grassmann and F. Peters, J. Chem. Phys. 113, 6774 (2000).

[60] R. Strey, P.E. Wagner, and Y. Viisanen, J. Phys. Chem. 98, 7748 (1994).

[61] K.N.H. Looijmans, P.C. Kriesel, and M.E.H. van Dongen, Exp. Fluids 15, 61 (1993).

[62] K.N.H. Looijmans and M.E.H. van Dongen, Exp. Fluids 23, 54 (1997).

[63] C.C.M. Luijten, O.D.E. Baas, and M.E.H. van Dongen, J. Chem. Phys. 106, 4152 (1997).

[64] C.C.M. Luijten, P. Peeters, and M.E.H. van Dongen, J. Chem. Phys. 111, 8535 (1999).

[65] B.E. Wyslouzil, G. Wilemski, M.G. Beals, and M.B. Frish, Phys. Fluids 6, 2845 (1994).

[66] R.A. Zahoransky, J. Höschele, and J. Steinwandel, J. Chem. Phys. 103, 9038 (1995).

[67] R.A. Zahoransky, J. Höschele, and J. Steinwandel, J. Chem. Phys. 110, 8842 (1999).

[68] B.E. Wyslouzil, C.H. Heath, J.L. Cheung, and G. Wilemski, J. Chem. Phys. 113, 7317 (2000).

[69] L.D. Landau and E.M. Lifshitz, Course of Theoretical Physics Vol 5 (Statistical Physics Part 1) and Vol 9 (Statistical Physics Part 2) (Pergamon Press, New York, 1980).

[70] D. Turnbull and J.C. Fisher, J. Chem. Phys. 17, 71 (1949). 
[71] W.C. Swope and H.C. Andersen, Phys. Rev. B 41, 7042 (1990);

[72] K.F. Kelton, A.L. Greer, and C.V. Thompson, J. Chem. Phys. 79, 6261 (1983).

[73] Ya.B. Zeldovich, Acta Physicochim URSS 18, 1 (1943).

[74] G. Shi, J.H. Seinfeld, and K. Okuyama, Phys. Rev. A 41, 2101 (1990); and 44, 8443 (1991).

[75] V.A. Shneidman, Phys. Rev. A 44, 8441 (1991) and references therein.

[76] H.A. Kramers, Physica 7, 284 (1940).

[77] J.E. Moyal, J. R. Stat. Soc. London Ser. B 11, 150 (1949).

[78] P. Hänggi, H. Grabert, P. Talkner, and H. Thomas, Phys. Rev. A 29, 371 (1984).

[79] J. Frenkel, Kinetic Theory of Liquids, (Clarendon, Oxford, 1946).

[80] F.C. Goodrich, Proc. R. Soc. London A 277, 167 (1964).

[81] B. Shizgal and J.C. Barrett, J. Chem. Phys. 91, 6505 (1989).

[82] M. Gitterman and G.H. Weiss, Physica A 170, 503 (1991).

[83] Y. Rabin and M. Gitterman, Phys. Rev. A 29, 1496 (1984).

[84] N.G. Van Kampen, Stochastic Processes in Chemistry and Physics, (North-Holland, Amsterdam, 1992).

[85] H. Grabert, P. Hänggi, and I. Oppenheim, Physica A 117, 300 (1983).

[86] V.A. Shneidman and P. Hänggi, Phys. Rev. E 49, 894 (1994).

[87] D.T. Wu, J. Chem. Phys. 97, 1922 (1992).

[88] F.G. Shi, Scr. Metal. et Mater. 30, 1195 (1994).

[89] J. Lothe and G. M. Pound, J. Chem. Phys. 36, 2080 (1962).

[90] H. Reiss, W.K. Kegel, and J.L. Katz, Phys. Rev. Lett. 78, 4506 (1997); J. Phys. Chem. A 102, 8548 (1998).

[91] S.L. Girshick and C-P Chiu, J. Chem. Phys. 93, 1273 (1990). 
[92] C. L. Weakliem and H. Reiss, J. Phys. Chem. 98, 6408 (1994).

[93] M.E. Fisher, Rep. Prog. Phys. 30, 615 (1967); and Physics 3, 255 (1967).

[94] S. Kiang, D. Stauffer, G.H. Walker, O.P. Puri, J.D. Wise, Jr., and E.M. Patterson, J. Atmos. Sci. 28, 1222 (1971).

[95] A. Dillmann and G.E.A. Meier, Chem. Phys. Lett. 160, 71 (1989).

[96] A. Dillmann and G.E.A. Meier, J. Chem. Phys. 94, 3872 (1991).

[97] R.C. Tolman, J. Chem. Phys. 17, 322 (1949).

[98] I.J. Ford, A. Laaksonen, and M. Kulmala, J. Chem. Phys. 93, 764 (1993).

[99] A. Laaksonen, I.J. Ford, and M. Kulmala, Phys. Rev. E 49, 5517 (1994).

[100] C.F. Delale and G.E.A. Meier, J. Chem. Phys. 98, 9850 (1993).

[101] V.I. Kalikmanov and M.E.H. van Dongen, Phys. Rev. E 47, 3532 (1993); and J. Chem. Phys. 103, 4250 (1995).

[102] B.N. Hale, Phys. Rev. A 33, 4156 (1986).

[103] R. McGraw and A. Laaksonen, Phys. Rev. Lett. 76, 2754 (1996).

[104] V. Talanquer, J. Chem. Phys. 106, 9957 (1997).

[105] L. Gránásy, Europhys. Lett. 24, 121 (1993).

[106] L. Gránásy and F. Iglói, J. Chem. Phys. 107, 3634 (1997).

[107] E.M. de Sá, E. Meyer, and V. Soares, J. Chem. Phys. 114, 8505 (2001).

[108] R. Lovett, J. Chem. Phys. 81, 6191 (1984).

[109] G. Narsimhan and E. Ruckenstein, J. Colloid Interface Sci. 128, 549 (1989).

[110] E. Ruckenstein and B. Nowakowski, J. Colloid Interface Sci. 137, 583 (1990).

[111] B. Nowakowski and E. Ruckenstein, J. Chem. Phys. 94, 1397 (1991).

[112] B. Nowakowski and E. Ruckenstein, J. Chem. Phys. 94, 8487 (1991). 
[113] D.W. Oxtoby in Fundamentals of Inhomogeneous Fluids, p. 407, (Marcel Dekker, New York, 1992).

[114] S.H. Bauer and D.J. Frurip, J. Phys. Chem. 81, 1015 (1977).

[115] C.F. Wilcox and S.H. Bauer, J. Chem. Phys. 94, 8302 (1991).

[116] S.H. Bauer, Y.-X. Zhand, and C.F. Wilcox, J. Chem. Phys. 110, 7926 (1999).

[117] G.K. Schenter, S.M. Kathmann, and B.C. Garrett, Phys. Rev. Lett. 82, 3484 (1999).

[118] R. Evans, Adv. Phys. 28, 143 (1979).

[119] J.W. Cahn and J.E. Hilliard, J. Chem. Phys. 28, 258 (1958).

[120] J.W. Cahn and J.E. Hilliard, J. Chem. Phys. 31, 688 (1959).

[121] D.W. Oxtoby and R.J. Evans, J. Chem. Phys. 89, 7521 (1988).

[122] X.C. Zeng and D.W. Oxtoby, J. Chem. Phys. 94, 4472 (1991).

[123] V. Talanquer and D.W. Oxtoby, J. Chem. Phys. 100, 5190 (1994).

[124] X.C. Zeng and D.W. Oxtoby, J. Chem. Phys. 104, 3726 (1996).

[125] V. Talanquer and D.W. Oxtoby, J. Chem. Phys. 106, 3673 (1997).

[126] V. Talanquer and D.W. Oxtoby, J. Chem. Phys. 112, 851 (2000).

[127] Y.C. Shen and D.W. Oxtoby, J. Chem. Phys. 104, 4233 (1996).

[128] V. Talanquer and D.W. Oxtoby, J. Chem. Phys. 109, 223 (1998).

[129] J.S. Langer, Ann. Phys. 41, 108 (1967).

[130] J.S. Langer, Ann. Phys. 54, 258 (1969).

[131] J.S. Langer, Physica 73, 61 (1974).

[132] J.S. Langer and L.A Turski, Phys. Rev. A 8, 3230 (1973).

[133] B. Senger, P. Schaaf, D.S. Corti, R. Bowles, J.-C. Voegel, and H. Reiss, J. Chem. Phys. 110, 6421 (1999).

[134] T.L. Hill, J. Chem. Phys. 23, 617 (1955). 
[135] F.H. Stillinger, J. Chem. Phys. 38, 1486 (1963).

[136] H. Reiss, A. Tabazadeh, and J. Talbot, J. Chem. Phys. 92, 1266 (1990).

[137] J.K. Lee, J.A. Barker, and F.F. Abraham, J. Chem. Phys. 61, 1221 (1974).

[138] H.M. Ellerby, C.L. Weakliem, and H. Reiss, J. Chem. Phys. 92, 1266 (1990).

[139] H.M. Ellerby and H. Reiss, J. Chem. Phys. 97, 5766 (1992).

[140] C.L. Weakliem and H. Reiss, J. Chem. Phys. 99, 5374 (1993).

[141] C.L. Weakliem and H. Reiss, J. Chem. Phys. 101, 2398 (1994).

[142] K.J. Oh, X.C. Zeng, and H. Reiss, J. Chem. Phys. 107, 1242 (1997).

[143] P. Schaaf, B. Senger, and H. Reiss, J. Phys. Chem. 101, 8740 (1997).

[144] M. Rao, B.J. Berne, and M.H. Kalos, J. Chem. Phys. 68, 1325 (1978).

[145] N.G. García and J.M.S. Torroja, Phys. Rev. Lett. 47, 186 (1981).

[146] G.M. Torrie and J.P. Valleau, Chem. Phys. Lett. 28, 578 (1974).

[147] J.S. van Duijneveldt and D. Frenkel, J. Chem. Phys. 96, 4655 (1992).

[148] P.R. ten Wolde and D. Frenkel, J. Chem. Phys. 109, 9901 (1998).

[149] P.R. ten Wolde, D.W. Oxtoby, and D. Frenkel, J. Chem. Phys. 111, 4762 (1999).

[150] K.J. Oh and X.C. Zeng, J. Chem. Phys. 110, 4471 (1999).

[151] V.K. Shen and P.G. Debenedetti, J. Chem. Phys. 111, 3581 (1999).

[152] H. Vehkamäki and I.J. Ford, J. Chem. Phys. 112, 4193 (2000).

[153] I. Kusaka, Z.-G. Wang, and J.H. Seinfeld, J. Chem. Phys. 108, 3416 (1998).

[154] I. Kusaka and D.W. Oxtoby, J. Chem. Phys. 110, 5249 (1999).

[155] H. Reiss and R.K. Bowles, J. Chem. Phys. 111, 9965 (1999).

[156] R.K. Bowles, J. Chem. Phys. 112, 1122 (2000). 
[157] H. Reiss and R.K. Bowles, J. Chem. Phys. 112, 1390 (2000).

[158] K.J. Oh and X.C. Zeng, J. Chem. Phys. 112, 294 (2000).

[159] D.J. McGinty, J. Chem. Phys. 58, 4733 (1973).

[160] D.I. Zhukhovitskii, J. Chem. Phys. 103, 9401 (1995).

[161] K. Laasonen, S. Wonczak, R. Strey, and A. Laaksonen, J. Chem. Phys. 113, 9741 (2000).

[162] For example: W.C. Swope and H.C. Andersen, Phys. Rev. B 41, 7042 (1990); L.A. Báez and P. Clancy, J. Chem. Phys. 102, 8183 (1995); L.S. Bartell, J. Phys. Chem. 99, 1080 (1998); K.Yasuoka and M. Matsumoto, J. Chem. Phys 109, 8451 (2000); and 109, 8463 (2000).

[163] P.R. ten Wolde, M.J. Ruiz-Montero, and D. Frenkel, J. Chem. Phys. 104, 9932 (1996).

[164] P.R. ten Wolde, M.J. Ruiz-Montero, and D. Frenkel, Faraday Discuss. 104, 93 (1996).

[165] P.R. ten Wolde, M.J. Ruiz-Montero, and D. Frenkel, Phys. Rev. Lett. 75, 2714 (1995).

[166] D. Chandler, J. Chem. Phys. 68, 2959 (1978).

[167] D. Chandler, Introduction to Modern Stastistical Mechanics, (Oxford University Press, 1987).

[168] C.H. Bennett, in Algorithms for Chemical Computation, edited by R.E. Christofferson (American Chemical Society, Washington. D. C. 1977).

[169] P. Schaaf, B. Senger, J.-C. Voegel, R.K. Bowles, and H. Reiss, J. Chem. Phys 114, 8091 (2001). 


\section{Chapter 2}

\section{Mesoscopic Nonequilibrium Thermodynamics Description of Nucleation Kinetics}

The first step in the kinetics of many phase transitions is, as we have seen, the formation of small embryos of the new phase within the bulk metastable substance. This is an activated process: a free energy barrier must be overcome in order to form embryos of a critical size, beyond which the new phase grows spontaneously.

Many nonequilibrium processes in nature may be described in terms of the crossing of a free energy barrier which separate two accessible states of the system, corresponding to the local minima at each side of the barrier. When the system localized at the left well acquires energy, it may surmount the barrier thus manifesting the characteristics of the state at the right well. Processes as thermal emission in semiconductors [1], chemical reactions [2], adsorption [3], nucleation [4], etc. share these common features and are usually referred to as activated processes.

It is interesting to emphasize the essential difference between activated processes and the second category of processes usually occurring in nonequilibrium situations, namely transport processes. The latter constitute the response to the application of an external force (or gradient) and may emerge even at very low values of the applied force. Contrarily, as commented previously, activated processes may only be induced whenever the applied perturbation exceeds a threshold value.

This difference has an important consequence. Whereas transport processes 
may exhibit a wide linear regime in which the external force and the established current are proportional, the regime in which activated processes develop is highly nonlinear. In this context, we can compare the linear Fourier, Fick or Ohm laws, in which the corresponding currents are proportional to the conjugated thermodynamic forces or gradients, with the exponential laws usually appearing in activated processes, as the nonlinear expression of the law of mass action giving the reaction rate of a chemical reaction in terms of its affinity.

The description of a mesoscopic process, like nucleation, requires the formulation of a kinetic equation tracing the evolution in time of the probability density of finding the system in a determined configuration. From this equation we can derive the evolution equations for the different moments providing expressions for the relaxation times, nucleation rates, etc., that can be contrasted with experiments.

The kinetics of homogeneous nucleation is habitually described from a theoretical point of view by means of kinetic equations of the Fokker-Planck type focused on the size distribution of the clusters present in the system. Due to the activated nature of the nucleation process, these equations are formally analogous to the ones arising from Kramers reaction-rate theory [5,6]. As mentioned in the previous chapter, these equations have been obtained mainly from three different procedures: as continuous approaches to a master equation; starting from microscopic kinetic models by considering the separation of a molecule from the cluster surface like a problem of Brownian diffusion through a barrier; or as the consequence of imposing a continuity equation for the probability density in the framework of field and density functional theories. The several approaches proposed to evaluate nucleation rates differ basically on the expressions for the drift and diffusion coefficients of the Fokker-Planck equation.

In spite of the great variety of proposed equations, up to now none of them has turned out to be fully satisfactory. In the previous chapter we have verified that equations obtained as continuous approximation to a master equation have the main drawback of not being able to reproduce some of the master equation characteristics (as the equilibrium distribution, the deterministic growth, the variance of the distribution or the short time behavior). Moreover, in all of them (except in the vicinities of the critical point) the coefficients are not fully determined, but rather their expression are postulated, or they are left in terms of the unspecified rates $k^{+}$and $k^{-}$. To estimate these rates an appropriate microscopic kinetic model is required. On the other hand, equations obtained from kinetic models base their validity on the correction of the microscopic model proposed for the dynamics of the cluster interface. Finally, the fieldtheory approach is restricted to the vicinity of the critical point and to situations 
where temporal dependencies do not exist.

Our purpose in this chapter is precisely to present a simple theoretical framework to analyze the kinetics of homogeneous nucleation. This scheme, based on mesoscopic nonequilibrium thermodynamics [7]-[15], provides a systematic method for the description of nonequilibrium processes occurring at a mesoscopic scale, such as nucleation. It has been applied successfully before to other problems like the adsorption kinetics of particles on surfaces [3], the description of chemical reactions [2] or the treatment of noise in interfaces of semiconductor devices [1]. This formalism allows the derivation of Fokker-Planck equations from firm thermodynamics and statistical mechanical foundations and not as a mere continuous approximation to a master equation. We want to show how the scheme we propose provides a complete nonequilibrium description of the process, and is able to abridge the gap between theory and simulations.

The chapter is structured as follows. In the Introduction, we justify the need of a framework to describe the kinetics of mesoscopic systems and activated processes. Section 2 is devoted to present the mesoscopic nonequilibrium thermodynamics theory. This scheme is particularized in Section 3 to analyze the kinetics of homogeneous nucleation. In this context, we will derive several Fokker-Planck equations describing the kinetics of the process from different perspectives. In particular, we will introduce new kinetic equations in terms of an order parameter or an improved hydrodynamic model, and we will also reproduce some of the kinetic equations proposed in the literature. In Section 4, we shall discuss the quasi-stationary case to show how in this case our approach manifests its consistency and overcomes some of the inherent difficulties of previous treatments. In Section 5, we outline how our theoretical framework can be easily connected with simulations. Finally, in the last section we summarize and comment on the main results of the theory we propose.

\subsection{Introduction}

Thermodynamics is a well-established theory for the description of systems at equilibrium. Time is an irrelevant variable in thermodynamics since it is restricted to changes which are infinitely slow (quasi-static) or so fast that equilibrium is reached immediately. Its scope, therefore, excludes the characterization of systems evolving in time.

Changes in the equilibrium state of a system are due to the presence of gradients, or in general of "thermodynamic forces" in the language of nonequilibrium thermodynamics. The response to those forces is the establishment of "fluxes" or "currents" trying to compensate their effect by returning the system 
to the equilibrium state.

The objective of nonequilibrium thermodynamics is precisely to relate the response of the system with the forces causing it. Nonequilibrium thermodynamics [8] provides a powerful tool to set up systematically the equations characterizing irreversible processes. To accomplish that objective, this theory resort and it is constructed over the firm foundations of thermodynamics. The crucial assumption allowing the application of thermodynamic concepts to out of equilibrium situations is the local equilibrium hypothesis. For it, one assumes that although the total system is not at equilibrium, a state of "local" equilibrium in which thermodynamics remains applicable, is established.

From the Gibbs equation (the first law) and the conservation laws for the mass, energy and momentum, one obtains the entropy balance equation from which one may identify the rate of entropy change due to nonequilibrium processes occurring in the system. That quantity, referred to as entropy production, plays a central role in the development of the theory. It contains all possible sources of dissipation and consists of a sum of products between fluxes (effects) and conjugated forces (causes, usually proportional to the gradients of system variables).

The second law of thermodynamics establishes that this entropy production should be positive. To fulfill this requirement, nonequilibrium thermodynamics then postulates linear relationships between fluxes and forces, known as constitutive equations. The proportionality coefficients are called phenomenological coefficients and permit the coupling between forces and fluxes of different nature, thus justifying the existence of cross-effects such as for instance the thermal-diffusion or the thermoelectric effects. These couplings are restricted by Onsager-Casimir and Curie principles, consequence of the symmetries of the underlying microscopic dynamics. Putting the conservation laws and the constitutive relations together, we obtain a complete set of differential equations characterizing the evolution of the system.

The nonequilibrium thermodynamic description of a system has some limitations. Its scope of application is restricted to macroscopic systems characterized in terms of state variables, such as temperature, pressure, chemical potential... In its standard formulation [8], nonequilibrium thermodynamics cannot describe the kinetics at the mesoscopic level, involving degrees of freedom as for example the velocity of a Brownian particle, the size of a polymer or a variable characterizing the state of a living cell.

Another restriction is due to the linear character of the constitutive relations. In principle, this linear approximation should only be appropriate when the magnitude of the gradients is small. In practice, linear relations have yet been 
proved to work reasonably well for many transport and relaxation processes even in presence of large gradients.

Nevertheless, for the class of activated processes, the linearity of the phenomenological laws does not constitute a satisfying approximation. A prototypical example is a chemical reaction. The standard nonequilibrium thermodynamics predicts a linear dependency of the reaction rate (the response of the system) in terms of the affinity (the corresponding force). But actually reaction rates evidence a highly nonlinear, exponential, dependency on the affinity, expressed by the law of mass action.

A mesoscopic reformulation of the standard nonequilibrium thermodynamics is able to overcome such difficulties, as described in the following section.

\subsection{Mesoscopic Nonequilibrium Thermodynamics}

The Mesoscopic Nonequilibrium Thermodynamics (MNET) theory constitutes a powerful, systematic and easy method to describe the kinetics of nonequilibrium processes occurring at a mesoscopic scale. It is based on the ideas introduced by Prigogine and Mazur [7] to extend nonequilibrium thermodynamics to systems with internal degrees of freedom. In its present formulation [16], it constitutes a generalization of the standard nonequilibrium thermodynamics able to describe the kinetics of mesoscopic entities in terms of arbitrary coordinates or degrees of freedom.

MNET is sustained over the grounds of nonequilibrium thermodynamics. But to be able to extend its scope up to the mesoscopic regime, concepts from statistical mechanics must be borrowed. In particular, the entropy which is the crucial quantity in nonequilibrium thermodynamics, is understood from its statistical perspective. The combination of the statistical definition of the entropy, which allows the description of a general system in terms of its probability density, with the systematic procedure of nonequilibrium thermodynamics, results in a powerful framework to describe the kinetics of a wide class of systems. This framework is outlined in the next subsection.

\subsubsection{General Derivation}

Let us assume that the state of a system is fully specified by a set of parameters $\gamma$, that we will call internal coordinates or internal degrees of freedom which describe the system locally. This arbitrary set of coordinates may represent the velocity of a particle, the orientation of a spin, the size of a macromolecule or whatever coordinate or order parameter whose values properly define the state 
of the system in a phase space. The characterization at the mesoscopic level is dictated by $f(\gamma, t)$, the probability density of finding the system at the state $\gamma \in(\gamma, \gamma+d \gamma)$ at time $t$.

We will assume that the evolution of the system can be described by means of a diffusion process over a potential landscape in the internal space. This landscape is conformed by the values of the energy associated to each configuration $\gamma$. The equilibrium state of the system is then characterized by the probability distribution function

$$
f_{e q} \sim \exp \left(\frac{-\Delta \mathcal{W}(\gamma)}{k_{B} T}\right)
$$

where $\Delta \mathcal{W}(\gamma)$ is the minimum reversible work required to create that state of the system [17], which is related to the maximum useful work which can be extracted from it, sometimes referred to as available energy [18] or availability [19]; $k_{B}$ is Boltzmann's constant, and $T$ is the temperature of the medium. Variations of the minimum work for a thermodynamic system are expressed as

$$
\Delta \mathcal{W}=\Delta U-T \Delta S+p \Delta V-\mu \Delta N+y \Delta Y+\ldots,
$$

where $U$ is the internal energy, $S$ the entropy, $V$ the volume, and $N$ the number of particles of the system, whereas $T, p$, and $\mu$ are the temperature, pressure and chemical potential of the environment. The term $y \Delta Y$ represents other kinds of work (electric, magnetic, surface work...) performed on the system, being $y$ the intensive parameter and $Y$ its conjugate extensive variable [20]. For the case of constant temperature, volume and number of particles, the minimum work corresponds to the Helmholtz free energy $F$. In general, that quantity reduces to the thermodynamics potentials by imposing to expression (2.2) the corresponding constraints [20] .

Having specified the statics of the system we will now derive the kinetic equation describing the evolution of the probability density. MNET applies the scheme of nonequilibrium thermodynamics to describe the dynamics of these mesoscopic degrees of freedom. The treatment of a diffusion process in the framework of nonequilibrium thermodynamics is extended to the case in which the relevant quantity is a probability density, defined in phase space, instead of a mass density. The starting point is then the formulation of the Gibbs equation for the entropy variations resulting from this diffusion process

$$
\delta S=-\frac{1}{T} \int \mu(\gamma) \delta f(\gamma, t) d \gamma
$$

which resembles the corresponding law proposed in nonequilibrium thermodynamics in terms of the mass density of particles. Here $\mu(\gamma)$ is a generalized 
chemical potential conjugated to the distribution function $f(\gamma, t)$.

The concept of entropy is not restricted to macroscopic systems in terms of mass density variables. In fact, for the description of a mesoscopic entity it is more convenient to resort to the definition of the entropy provided by Statistical Mechanics. It is precisely the connection between the thermodynamic and statistical mechanics definition of the entropy which facilitates the identification of the chemical potential.

The statistical mechanics definition of the entropy is given through the Gibbs entropy postulate [8]

$$
S=-k_{B} \int f(\gamma, t) \ln \frac{f(\gamma, t)}{f_{e q}(\gamma)} d \gamma+S_{e q}
$$

where $S_{e q}$ is the value of the entropy at equilibrium and $f_{e q}(\gamma)$ is the equilibrium distribution given through Eq. (2.1). Taking variations in the previous equation one obtains

$$
\delta S=-k_{B} \int \delta f(\gamma, t) \ln \frac{f(\gamma, t)}{f_{e q}(\gamma)} d \gamma+\delta S_{e q}
$$

where the variations of the equilibrium entropy are given by

$$
\delta S_{e q}=-\frac{1}{T} \int \mu_{e q} \delta f(\gamma, t) d \gamma
$$

and $\mu_{e q}$ is the value of the chemical potential at equilibrium. Comparison of Eq. (2.5) with the Gibbs equation (2.3) yields the expression for the chemical potential

$$
\mu(\gamma, t)=k_{B} T \ln \frac{f(\gamma, t)}{f_{e q}(\gamma)}+\mu_{e q}
$$

Quite generally, the evolution of the probability density in the internal space is governed by the continuity equation

$$
\frac{\partial f}{\partial t}=-\frac{\partial J}{\partial \gamma}
$$

where $J(\gamma, t)$ is a current or density flux in the internal space which has to be specified. To obtain its value, one proceeds to derive the expression of the entropy production $\sigma=d S / d t$ which follows from the continuity equation (2.8) and the Gibbs equation (2.3). One then arrives at

$$
\sigma=-\frac{1}{T} \int J(\gamma, t) \frac{\partial \mu}{\partial \gamma}(\gamma, t) d \gamma
$$


where a partial integration has been performed, with the assumption that the diffusion current vanishes at the initial and final states of the process. This quantity has the usual form of a sum of flux-force pairs.

Nonequilibrium thermodynamics assumes linear dependency between fluxes and forces and establishes linear phenomenological relations between them. In our case, following this scheme, we obtain the desired expression for the current

$$
J(\gamma, t)=-\frac{L(\gamma)}{T} \frac{\partial \mu}{\partial \gamma}(\gamma, t)
$$

where $L(\gamma)$ is a phenomenological coefficient which may in general depend on the internal coordinate. To derive this expression, locality in the internal space has also been taken into account, for which only fluxes, $J(\gamma, t)$, and forces, $T^{-1} \partial \mu(\gamma, t) / \partial \gamma$, with the same value of $\gamma$ become coupled.

The resulting kinetic equation then follows by substituting Eqs. (2.10) and (2.7) into the continuity equation (2.8)

$$
\frac{\partial f}{\partial t}=\frac{\partial}{\partial \gamma}\left(D f_{e q} \frac{\partial}{\partial \gamma} \frac{f}{f_{e q}}\right)
$$

where we have defined the diffusion coefficient as

$$
D(\gamma) \equiv \frac{k_{B} L(\gamma)}{f}
$$

This equation, which in view of Eq. (2.1) can also be written as

$$
\frac{\partial f}{\partial t}=\frac{\partial}{\partial \gamma}\left(D \frac{\partial f}{\partial \gamma}+\frac{D}{k_{B} T} \frac{\partial \Delta \mathcal{W}}{\partial \gamma} f\right)
$$

is the Fokker-Planck equation accounting for the evolution of the probability density in $\gamma$-space.

Under the conditions for which $\Delta \mathcal{W}=\Delta G=\Delta H-T \Delta S$, this equation transforms into the Fokker-Planck equation for a system in the presence of a free energy barrier. One then obtains

$$
\frac{\partial f}{\partial t}=\frac{\partial}{\partial \gamma} \cdot\left(D \frac{\partial f}{\partial \gamma}+\frac{D}{k_{B} T} \frac{\partial \Delta G}{\partial \gamma} f\right)
$$

Other cases of interest concern different thermodynamic potentials. For instance, a particularly interesting situation is the case of a purely entropic barrier, often encountered in soft-condensed matter and biophysics, and discussed in detail in Ref. [16]. 
The general form of Eq. (2.11), in which the equilibrium distribution function does not need to be specified and is given in general by Eq. (2.1), makes that result applicable to a great diversity of situations. The method used in this section then offers a common formalism able to analyze the dynamics of any system at the mesoscopic scale.

Finally, to render the Fokker-Planck dynamics complete, we need to specify the value of the diffusion coefficient in the internal space, $D(\gamma)$, as well as to determine the form of the barrier, $\Delta \mathcal{W}(\gamma)$. Both quantities can obviously be borrowed from well-established theories. However, one can alternatively obtain these quantities from simulations.

The value of the free energy barrier is related to the equilibrium distribution (2.1) which can be evaluated from Monte Carlo or molecular dynamics simulations as proposed by van Duijneveldt and Frenkel in Ref. [21]-[23] for the specific case of homogeneous nucleation.

To determine the diffusion coefficient $D(\gamma)$ one can use the Landau-Lifshitz fluctuating hydrodynamics [17],[2]. This theory, originally formulated for transport processes and subsequently extended to activated processes [3], proposes the decomposition of the total current into a systematic and random contributions. In our interpretation of the nucleation process as a diffusion along an internal coordinate, we can apply that formalism to incorporate fluctuations to the former analysis. The total diffusion current splits up into systematic and random contributions in the form

$$
J(\gamma, t)=J^{s}(\gamma, t)+J^{r}(\gamma, t) .
$$

The systematic part $J^{s}(\gamma, t)$ matches expression (2.8) whereas the random part $J^{r}(\gamma, t)$ constitutes a Gaussian white noise stochastic process with zero mean and fluctuation-dissipation theorem given by

$$
\left\langle J^{r}(\gamma, t) J^{r}\left(\gamma^{\prime}, t^{\prime}\right)\right\rangle=2 k_{B} L \delta\left(\gamma-\gamma^{\prime}\right) \delta\left(t-t^{\prime}\right)=2 D f \delta\left(\gamma-\gamma^{\prime}\right) \delta\left(t-t^{\prime}\right) .
$$

Integration of Eq. (2.16) with respect to time and to the internal coordinate then leads to an expression of the diffusion coefficient in terms of the correlations of the random current

$$
D(\gamma, t)=\frac{1}{f(\gamma, t)} \iint_{0}^{\infty}\left\langle J^{r}(\gamma, 0) J^{r}\left(\gamma^{\prime}, t\right)\right\rangle d t d \gamma^{\prime} .
$$

This equation constitutes a Green-Kubo formula and can be used to determine the diffusion coefficient in the internal space from realizations of the underlying 
stochastic process. For each interpretation of the degree of freedom, the later equation could be transformed in order to be evaluated by means of simulations. A concrete example for the nucleation case is given in Section 2.5. Consequently, our scheme becomes in this way completely solvable at least at the simulation level.

The incorporation of fluctuations in the internal space indicated previously implies the formulation of a more general kinetic equation [11],[13]. Taking into account the random diffusion current in Eq. (2.8) this expression transforms into

$$
\frac{\partial f}{\partial t}=\frac{\partial}{\partial \gamma}\left\{D(\gamma, t) \frac{\partial f}{\partial \gamma}+\frac{D}{k_{B} T} \frac{\partial \Delta \mathcal{W}}{\partial \gamma} f\right\}+F^{r}
$$

containing a random source $F^{r}(\gamma, t)=-\frac{\partial}{\partial \gamma} J^{r}(\gamma, t)$. This equation, of the Fokker-Planck-Langevin type, expresses the fact that the distribution function may fluctuate around a mean value solution of the kinetic equation.

The discussion of the stochastic kinetic equations ends our presentation of the MNET.

\subsection{MNET Approach to Nucleation Kinetics}

Once established the general MNET framework, our aim in this section is to particularize the method to the case of nucleation. Adopting the previous picture of the evolution of a mesoscopic system, the process of nucleation and growth of the new phase can be viewed as a diffusion process through the free energy barrier $\Delta \mathcal{W}(\gamma)$ that separates the metastable (vapor or liquid) phase corresponding to $\gamma_{1}$ from the new stable (liquid or crystal) phase characterized by $\gamma_{2}$ (see Fig. 2.1).

The formalism we have proposed then enables us to formulate a general Fokker-Planck equation in terms of an arbitrary mesoscopic coordinate $\gamma$ specifying the state of the system. Thus, one of its inherent advantages comes from the fact that it is not necessarily restricted to give a vision of the nucleation only focused on the kinetics of clusters. Contrarily, a suitable choice of the internal coordinate will lead to the Fokker-Planck equation corresponding to a different description of the nucleation process.

In this section we shall particularize this equation for different interpretations of the mesoscopic coordinate, suitable to describe nucleation. In this way, we will see how we can propose new Fokker-Planck equations for alternative descriptions of nucleation, in terms for instance of a global crystallization order 


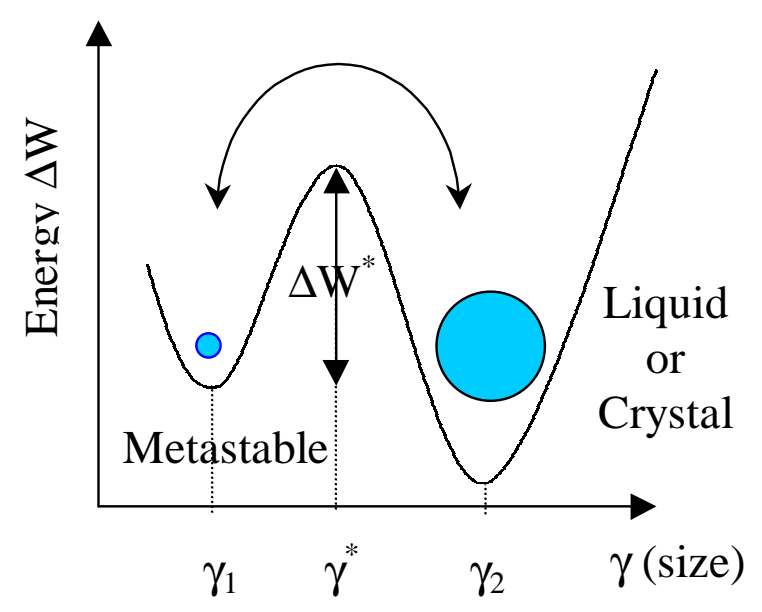

Figure 2.1: Nucleation as a diffusion over an energy landscape $\Delta \mathcal{W}(\gamma)$. The internal coordinate $\gamma$ may for instance represent the size of a cluster.

parameter $Q$ or from an hydrodynamic perspective, and also how to reproduce the already existing ones.

\subsection{1 $\gamma$ is Related to the Number of Molecules in a Cluster}

When nucleation proceeds by the formation of spherical clusters, the process can be completely characterized by a single coordinate. This coordinate may represent for instance the number of monomers in a cluster or the cluster size.

Let us consider as internal coordinate the number of monomers that constitute a cluster, $n$. In this case, $f$ would represent the (time-dependent) clusters size distribution, and $\Delta \mathcal{W}(\gamma) \equiv \Delta G(n)$ would be the free energy needed to form a cluster of size $n$. Assuming that $n$ varies in a continuous way, equation (2.11) yields

$$
\frac{\partial f}{\partial t}=\frac{\partial}{\partial n}\left(D_{n} f_{e q} \frac{\partial}{\partial n} \frac{f}{f_{e q}}\right)
$$

which resembles the Fokker-Planck equation (1.29) obtained as continuous approximation to a master equation. To complete the analogy, the diffusion coefficient $D_{n}$ given in general by Eq. (2.12) would be identified with $k^{+}$.

It is worth pointing out that our formalism recovers in a natural and direct 
way (without additional assumptions) the correct equilibrium distribution. It is straightforward to verify that the equilibrium distribution (2.1) is indeed a stationary solution of equation (2.11) satisfying the condition $J=0$.

\subsection{2 $\gamma$ Represents an Order Parameter}

A possible way of characterizing the nucleation process for the case of crystallization, which has recently been used by Frenkel and coworkers in computer simulations (see Refs. [22],[23]), is by means of an order parameter $Q$ that describes the global degree of crystallization of the system. In these simulations, the nucleation rate is calculated from a phenomenological rate equation using linear response theory.

One may wonder why they use linear response theory instead of appealing to the usual nucleation theories or even to the prototypical theory of activated processes: the Kramers theory. In fact, in their simulations [22],[23] they report on a diffusive behavior that seems to corroborate the validity of Kramers picture. Additionally, they also employ Kramers equation in order to obtain reasonably estimations of the time evolution of the system.

In Ref. [24] the authors themselves expose the reasons why they do not use Kramers theory. First, they assert that it was originally formulated for the single particle diffusion problem and therefore its validity is not guaranteed in the case when the reaction coordinate is a global one. On the other hand, they point out that the diffusion coefficient, assumed to be constant in Kramers picture, may depend on the value of the reaction coordinate, and moreover its expression may be non-trivial. Finally, they affirm that a Fokker-Planck description of nucleation processes does not take into account hydrodynamic effects that may be very important in this type of barrier-crossing processes.

Within our framework we can easily propose a theoretical description of homogeneous nucleation in terms of a global order parameter as the one used by Frenkel and coworkers in their simulations [25].

According to our theory, the underlying Fokker-Planck equation describing the kinetics of this process in terms of the variable $Q$, considered as the internal degree of freedom, is given by

$$
\frac{\partial f}{\partial t}=\frac{\partial}{\partial Q}\left(D(Q, t) \frac{\partial f}{\partial Q}+\frac{D(Q, t)}{k_{B} T} \frac{\partial \Delta G}{\partial \gamma} f\right)
$$

where $\Delta G(Q)$ is the free-energy of formation of the state with crystallinity $Q$, and the expression of the diffusion coefficient is, following Eq. (2.12), 


$$
D(Q, t)=\frac{k_{B} L(Q)}{f(Q, t)}
$$

If the internal coordinate represents an order parameter associated to the whole crystallinity, the interpretation of $f$ is slightly different. Now $Q$ is a variable that characterizes the global state of the system. Therefore, the internal space associated to this variable would be the set of all the feasible replicas of the system in a given "macrostate". From this point of view, $f$ would represent the non-equilibrium distribution of this ensemble whose meaning would be the fraction of systems with value of the internal coordinate equal to $Q$ at time $t$. The quantity $f d Q$ would then be interpreted as the probability that the order parameter of our system (a single realization of this non-equilibrium ensemble) has a value in the interval $(Q, Q+d Q)$ at time $t$.

The Fokker-Planck equation (2.20) we have introduced provides a complete description of the process, it includes the proper transition rate expression as a quasi-stationary limit (as we shall demonstrate in Section 2.5) and it overcomes the reservations exposed by Frenkel et al. [25]

\subsection{3 $\gamma$ is the Cluster Radius}

If we consider the coordinate $\vec{r}$, corresponding to the position of a monomer in a cluster of radius $R$, as the internal degree of freedom and we work in spherical coordinates, we immediately recover the Smoluchowski equation proposed by Ruckenstein and coworkers [26]-[29]

$$
\frac{\partial f(\vec{r}, t)}{\partial t}=\vec{\nabla}_{\vec{r}} \cdot\left(D(\vec{r}, t) \vec{\nabla}_{\vec{r}} f(\vec{r}, t)+\frac{D(\vec{r}, t)}{k_{B} T} f(\vec{r}, t) \vec{\nabla}_{\vec{r}} \phi(\vec{r})\right) .
$$

Notice that now, since the phenomenological coefficient defined in Eq. (2.12) is in general a function of the internal coordinate, the diffusion coefficient will depend on the radial distance $\vec{r}$. In this case, $f$ would represent the number density of monomers at $\vec{r}$, and $\phi$ the energy barrier to overcome in order to leave the cluster.

\subsection{4 $\gamma$ is the Set of Degrees of Freedom of the System}

If instead of using a single coordinate we carry out a complete description in terms of all the system's degrees of freedom $\{\eta\}$, characterizing a given configuration, we recover the form of the Fokker-Planck equation proposed by Langer [30]-[32] 


$$
\frac{\partial f(\{\eta\}, t)}{\partial t}=\sum_{i, j} \frac{\partial}{\partial \eta_{i}}\left(D_{i j}\left(\frac{\partial f}{\partial \eta_{j}}+\frac{\partial \Delta \Omega}{\partial \eta_{j}} \frac{f}{k T}\right)\right),
$$

where now $D_{i j}=\frac{k_{B} L_{i j}(\{\eta\})}{f(\{\eta\}, t)}$. In this case, $f(\{\eta\}, t)$ corresponds to the probability density associated to the $\{\eta\}$ configuration and $\Delta \mathcal{W}(\{\eta\}) \equiv \Delta \Omega(\{\eta\})$ is the free energy functional.

\subsection{5 $\gamma$ is the Density $\rho(\mathbf{r})$}

Due to the use of the capillarity approximation in the classical treatment of nucleation, clusters which are the embryos of the new phase can be characterized only through its molecular content or equivalently its radius. However, simulations show that this simplified description of a cluster is not always sufficient, since in fact clusters often present a diffuse interface.

A more detailed characterization of a cluster could then be given not only in terms of its molecular content, but through its density profile $\rho(\mathbf{r})$. This is the starting point of density functional calculations [33]-[35], which focus on evaluation of the free energy functional $\Omega[\rho(\mathbf{r})]$ required to form this cluster. However, the nonequilibrium kinetic description of the process lies in general outside of the scope of these approaches.

Within our framework, we can also propose a kinetic description of the process in terms of the density functional. In this case, the internal coordinates are the density profile $\rho(\mathbf{r})$, and also the position variable $\mathbf{r}$ which is integrated to render the coarse-grained description. The resulting evolution equation, following Eq. (2.13) is

$$
\frac{\partial P}{\partial t}=\int \frac{\delta}{\delta \rho(\mathbf{r})}[\nabla \cdot \underline{D} \cdot \nabla]\left\{\frac{\delta}{\delta \rho(\mathbf{r})}+\frac{1}{k_{B} T} \frac{\delta \Delta \Omega}{\delta \rho(\mathbf{r})}\right\} P d \mathbf{r}
$$

where $P[\rho(\mathbf{r})]$ is the distribution functional of the density and $\underline{D}[\rho(\mathbf{r})]$ is the kinetic factor. However in this situation an hydrodynamic description, as the one developed in the following subsection, is more appropriate.

\subsubsection{Hydrodynamic Description}

The description of condensation in terms of a density profile has some inherent restrictions associated to the length-scale requirements underlying a coarsegrained construction. In particular, densities should be smooth functions of space and time, and vary over scales which are much larger than any microscopic scale. Therefore, this description requires a semi-macroscopic size of 
the fluctuations involving a large number of molecules. This requirement is satisfied when the phase transformation occurs in the vicinity of the critical point. In that case, a hydrodynamic description in terms of the local number density $\rho(\mathbf{r})$ and momentum $\mathbf{g}(\mathbf{r})$, as the one proposed by Langer and Turski [36] becomes appropriate. Far from the critical point, the fluctuations are not semi-macroscopic, and the validity of this type of modeling is not guaranteed.

Let us outline how a hydrodynamic description could be easily obtained inside the MNET framework [37]. The starting point is the consideration of the system as a continuum divided in tiny cells. In each cell we define a mass density $\rho(\mathbf{r})$ and a momentum density $\mathbf{g}(\mathbf{r})=\rho \mathbf{v}$, with $\mathbf{r}$ being the position vector of a cell and $\mathbf{v}$ the velocity field. For simplicity, we will introduce the compact notation $\{\underline{\Gamma}\} \equiv\{\rho, \mathbf{g}\}$.

Let us consider $\widehat{P}(\{\underline{\Gamma}\}, t)$ as the distribution functional in phase space. Related to this distribution functional we introduce the phase space entropy $S(\widehat{P}(\{\underline{\Gamma}\}, t))$, which satisfies the Gibbs equation analogous to Eq. (2.3)

$$
\triangle S=-\frac{1}{T} \int \mu(\{\underline{\Gamma}\}, t) \triangle \widehat{P}(\{\underline{\Gamma}\}, t) \delta \underline{\Gamma} .
$$

Here $\mu$ represents the chemical potential conjugated to the distribution functional, whose expression

$$
\mu=\mu_{l e q}+k T \ln \frac{\widehat{P}}{\widehat{P}^{l e q}}
$$

can be obtained with the help of the Gibbs entropy postulate, as explained previously. In the former formula

$$
\widehat{P}^{l e q}=\exp \left\{-\frac{1}{k_{B} T}\left[\mu_{e q}+\Omega_{K}\{\underline{\Gamma}\}+\Omega\{\rho\}\right]\right\}
$$

is the distribution functional at local equilibrium, and $\mu_{e q}$ the value of the chemical potential at local equilibrium. Moreover, the coarse-grained free energy occurring in the equilibrium distribution consist of a kinetic part, given by

$$
\Omega_{K}\{\underline{\Gamma}\}=\frac{1}{2} \int \frac{\mathbf{g}(\mathbf{r})^{2}}{\rho(\mathbf{r})} d \mathbf{r},
$$

plus an interaction term $\Omega\{\rho\}$ which represents the coarse-grained free-energy density functional.

We now assume that the distribution functional evolves according to the continuity equation

$$
\frac{\partial \widehat{P}}{\partial t}+\int\left(\frac{\delta}{\delta \rho} \dot{\rho} \widehat{P}+\frac{\delta}{\delta \mathbf{g}} \cdot \dot{\mathrm{g}} \widehat{P}\right) d \mathbf{r}=-\int \frac{\delta}{\delta \mathbf{g}} \cdot \mathbf{J}_{g} d \mathbf{r}
$$


where the dot over the field variables indicates time derivative and $\mathbf{J}_{\mathbf{g}}$ is a diffusive current in momentum space.

From Eqs. (2.25) and (2.29) we can obtain the rate of the entropy variation that is written as

$$
\frac{\partial S}{\partial t}=\int \mu\left(\frac{\delta}{\delta \rho} \dot{\rho} \widehat{P}+\frac{\delta}{\delta \mathbf{g}} \cdot \dot{\mathbf{g}} \widehat{P}\right) d \mathbf{r}+\sum .
$$

In this expression, the first term on the right hand side is the rate at which entropy is supplied to the system by its surroundings through the external constrains. The second contribution corresponds to the entropy produced inside the system due to the irreversible processes, whose value is

$$
\sum=-\frac{1}{T} \iint \mathbf{J}_{g} \cdot \frac{\delta \mu}{\delta \mathbf{g}} \delta \underline{\Gamma} d \mathbf{r}
$$

where a partial integration has been performed. This entropy production has the usual form of flux-force pairs from which we can infer the phenomenological relation

$$
\mathbf{J}_{g}=-\frac{1}{T} \int \underline{L}\left(\mathbf{r}, \mathbf{r}^{\prime}\right) \cdot \frac{\delta \mu}{\delta \mathbf{g}} d \mathbf{r}^{\prime}
$$

where the phenomenological coefficients $\underline{L}$ satisfy the Onsager relations $\underline{L}\left(\mathbf{r}, \mathbf{r}^{\prime}\right)=$ $\underline{L}\left(\mathbf{r}^{\prime}, \mathbf{r}\right)^{\dagger}$, in which ${ }^{\dagger}$ stands for the Hermitian conjugate. By computing the functional derivative and assuming locality in the coordinates, i.e. $\underline{L}\left(\mathbf{r}, \mathbf{r}^{\prime}\right)=$ $\underline{L}(\mathbf{r}) \delta\left(\mathbf{r}-\mathbf{r}^{\prime}\right)$, one obtains

$$
\mathbf{J}_{g}=-\underline{H}(\mathbf{r}) \cdot\left(k_{B} T \frac{\delta}{\delta \mathbf{g}}+\frac{\delta \Omega_{K}}{\delta \mathbf{g}}\right) \widehat{P}
$$

where $\underline{H}(\mathbf{r}) \equiv \underline{L}(\mathbf{r}) / T \widehat{P}$ can be interpreted as a mobility tensor. Its expression follows from the Navier-Stokes equation [38]

$$
\underline{H}(\mathbf{r})=-\eta\left(\frac{1}{3} \nabla \nabla+\mathbb{1} \nabla^{2}\right)-\xi \nabla \nabla
$$

with $\eta$ and $\xi$ being the shear and bulk viscosities, respectively, and $\mathbb{1}$ the unit tensor.

By introducing the current we have obtained in Eq. (2.33) into the continuity equation (2.29) and using the expressions of $\dot{\rho}$ and $\dot{\mathbf{g}}$ given by the reversible part of the Navier-Stokes equation [38], this yields

$$
\begin{aligned}
\frac{\partial \widehat{P}}{\partial t}=\int & \left\{\frac{\delta}{\delta \rho} \nabla \cdot \mathbf{g}+\frac{\delta}{\delta \mathbf{g}} \cdot\left[\rho \nabla \frac{\delta \Omega}{\delta \rho}+\nabla \cdot \frac{\mathbf{g g}}{\rho}\right]\right. \\
& \left.+\frac{\delta}{\delta \mathbf{g}} \cdot \underline{H}(\mathbf{r}) \cdot\left[k_{B} T \frac{\delta}{\delta \mathbf{g}}+\frac{\delta \Omega_{K}}{\delta \mathbf{g}}\right]\right\} \widehat{P} d \mathbf{r}
\end{aligned}
$$


which constitutes the functional Fokker-Planck equation for the probability distribution functional $\widehat{P}(\{\underline{\Gamma}\}, t)$.

If we choose as interaction term $\Omega\{\rho\}$ the Cahn-Hilliard free-energy functional [35] given by Eq. (1.36) and discard the last term on the previous equation, then Eq. (2.35) resembles the one proposed by Langer and Turski [36]. However, our formalism provides a more complete hydrodynamic description of nucleation, since it accounts for the irreversible transference of moment and viscous effects represented by the last term of Eq. (2.35).

\subsection{The Quasi-Stationary Case}

In the previous section, we have shown how to derive different forms of FokkerPlanck equations. Our task now will be to ascertain if the equations we have obtained, not only have the appropriate form, but are also physically consistent. To this purpose, and for the sake of simplicity, we will focus our attention on the clusters size distribution equation (2.19).

Most of proposed Fokker-Planck equations are just different continuous diffusive approximations to a discrete master equation. Thus, the rightness of this kind of equations is evaluated in terms of the accuracy of the approximation. In particular, the two main requirements that a Fokker-Planck equation must satisfy is that it reproduces the equilibrium distribution and the deterministic growth rate $\dot{n}$ [39]; in other words, it must appropriately describe nucleation and growth.

The Fokker-Planck equation we present has been derived directly and not as a continuous approach to a master equation. Therefore, in general we do not have an underlying master equation model to which our expression should be adjusted and that serve us as a criterion to judge its accuracy.

However, we have shown that, by construction, our equation always guarantees the correct equilibrium distribution. Moreover, a case exists in which we can build up a master equation to evaluate the correctness of our results. It is the quasi-stationary case, corresponding to high nucleation barriers.

When the height of the nucleation barrier is large enough as compared with thermal energy, the system achieves a quasi-stationary state characterized by the current

$$
J(n, t)=J(t)\left\{\theta\left(n-n_{1}\right)-\theta\left(n-n_{2}\right)\right\},
$$

where $\theta$ is the step function. This form implies that equilibrium is reached independently at each barrier side, consequently the chemical potential will be 
uniform

$$
\mu(n, t)=\mu\left(n_{1}, t\right) \theta\left(n^{*}-n\right)+\mu\left(n_{2}, t\right) \theta\left(n-n^{*}\right) .
$$

Here $n^{*}$ specifies the size of the critical cluster.

Substituting these two equations in (2.10) and integrating with respect to the coordinate $n$, we obtain

$$
J(t)=\frac{D\left(n^{*}\right)}{n_{2}-n_{1}}\left(f\left(n_{1}, t\right) e^{-\frac{\Delta G\left(n^{*}\right)-\Delta G\left(n_{1}\right)}{k_{B} T}}-f\left(n_{2}, t\right) e^{-\frac{\Delta G\left(n^{*}\right)-\Delta G\left(n_{2}\right)}{k_{B} B^{T}}}\right) .
$$

This is the expression of the nucleation rate in the quasi-stationary case. It is important to highlight that the value of the rate $J(t)$ depends exponentially on the nucleation barrier, thus confirming the activated nature of the process. The ability of MNET in describing the kinetics of activated processes is then corroborated.

If the nucleation barrier is high enough in such a way that a quasi-stationary state is reached, the clusters distribution of intermediate sizes does not vary in time, so that only the states corresponding to $f\left(n_{1}, t\right)$ and $f\left(n_{2}, t\right)$ matter. The value $n_{1}$ represents the smaller cluster distinguishable from equilibrium fluctuations in the metastable phase and $n_{2}$ corresponds to a post-critical stable cluster. Clusters sizes $n_{1}$ and $n_{2}$ ( where $n_{1}<n^{*}<n_{2}$ ) are chosen such that for $n<n_{1}, f(n)=f_{e q}(n)$ and for $n>n_{2}, f(n)=0$. These boundary conditions are habitual in classical treatments [40] and final results do not strongly depend on the explicit values of $n_{1}$ and $n_{2}$ [41]. If the intermediate sizes distribution is constant, it implies that the lost of one $n_{1}$ cluster supposes, after jumping the energy barrier, the formation of one post-critical nucleus. Conversely, the gain of $n_{1}$ clusters is due to the disappearance of a post-critical cluster.

Accordingly, the master equation for this quasi-stationary situation has the following form:

$$
\begin{gathered}
\frac{d f\left(n_{1}, t\right)}{d t}=k_{21} f\left(n_{2}, t\right)-k_{12} f\left(n_{1}, t\right)=-J(t) \\
\frac{d f\left(n_{2}, t\right)}{d t}=k_{12} f\left(n_{1}, t\right)-k_{21} f\left(n_{2}, t\right)=J(t)
\end{gathered}
$$

where $k_{12}$ and $k_{21}$ are the forward and backward rates. The forward rate $k_{12}$ is simply the probability that a cluster $n_{1}$ disappears, or equivalently, that it surpasses the nucleation barrier. Therefore, $k_{12}$ should be proportional to the Boltzmann factor associated to the height of the barrier 


$$
k_{12}=v e^{-\frac{\Delta G\left(n^{*}\right)-\Delta G\left(n_{1}\right)}{k_{B} T}} .
$$

Similarly, for the rate $k_{21}$ one has

$$
k_{21}=v e^{-\frac{\Delta G\left(n^{*}\right)-\Delta G\left(n_{2}\right)}{k_{B} T}}
$$

Notice that the constant $v$ enters both expressions of $k_{12}$ and $k_{21}$ in order to guarantee that in equilibrium the flow vanishes. This is the requirement to fulfill detailed balance.

Therefore, the nucleation rate $J(t)$ obtained from the master equation is given by

$$
J(t)=v\left(f\left(n_{1}, t\right) e^{-\frac{\Delta G\left(n^{*}\right)-\Delta G\left(n_{1}\right)}{k_{B} B^{T}}}-f\left(n_{2}, t\right) e^{-\frac{\Delta G\left(n^{*}\right)-\Delta G\left(n_{2}\right)}{k_{B} T}}\right) .
$$

Identifying $v$ with the factor $\frac{D^{*}}{n_{2}-n_{1}}$ in Eq. (2.38), we see that at least in the case of high nucleation barriers, our diffusive equation reproduces the steadystate nucleation rate. Moreover, we are going to prove that our formalism also recovers all the distribution moments of the master equation model.

By introducing (2.36) into the continuity equation (2.8) we obtain the following expression

$$
\frac{\partial f(n, t)}{\partial t}=-J(t)\left\{\delta\left(n-n_{1}\right)-\delta\left(n-n_{2}\right)\right\}
$$

which yields the evolution equation for the $r$-moment $(r=1,2,3 \ldots)$

$$
\frac{d<n^{r}>}{d t}=\left(n_{2}^{r}-n_{1}^{r}\right) J(t) .
$$

It is immediate to realize that this expression agrees with the corresponding one evaluated from the set of master equations (2.39)-(2.40).

Therefore, we have proved the validity of our equation in the quasi-stationary case, in the sense that it satisfies the two rightness criteria required to make our approach consistent, and moreover reproduces the same results obtained from a master equation. If the height of the barrier is low, the quasi-stationary hypothesis is not longer valid. This feature invalidate the considerations we have made in this section. 


\subsection{Connection with Simulations}

In the last years, computer simulations have also become a useful tool to study the kinetics of crystal nucleation. However, in order to analyze the results of simulations the most common theoretical treatments for nucleation processes are not generally employed.

The objective of this section is to illustrate how to transform the GreenKubo expression (2.17) for the diffusion coefficient into a formula suitable for computer simulations. For the sake of concreteness and simplicity, we are going to focus our attention in the description of nucleation in terms of a global order parameter, employed by Frenkel and coworkers in their simulations [22], and described in Section 2.3.2. First, we will show how, in the quasi-stationary case, we can recover explicitly the reactive flux expression used by these authors to evaluate nucleation rates, and derived in the Appendix. Subsequently, we will analyze the general case.

\subsubsection{Quasi-Stationary Case}

When the undercooling or the supersaturation is low enough, the energy barrier to surpass in the nucleation process is large as compared with thermal energy. In this high barrier situation, as we mentioned in the previous section, the system achieves a quasi-stationary state characterized by an uniform current

$$
J(Q, t)=J(t)\left\{\theta\left(Q-Q_{1}\right)-\theta\left(Q-Q_{2}\right)\right\},
$$

a chemical potential which equilibrates independently at each barrier side

$$
\mu(Q, t)=\mu\left(Q_{1}, t\right) \theta\left(Q_{0}-Q\right)+\mu\left(Q_{2}, t\right) \theta\left(Q-Q_{0}\right),
$$

and a probability density

$f(Q, t)=f\left(Q_{1}, t\right) e^{-\frac{\Delta G(Q)-\Delta G\left(Q_{1}\right)}{k_{B} T}} \theta\left(Q_{0}-Q\right)+f\left(Q_{2}, t\right) e^{-\frac{\Delta G(Q)-\Delta G\left(Q_{2}\right)}{k_{B} T}} \theta\left(Q-Q_{0}\right)$

which resembles the corresponding Eqs. (2.36)-(2.37) but are now expressed in terms of $Q$. In the previous equations $Q_{0}$ represents the position of the top of the barrier.

On the other hand the diffusive current (2.10) can be written in a more convenient way as

$$
J(Q, t)=-D(Q, t) e^{-\Delta G / k_{B} T} \frac{\partial}{\partial Q} e^{\mu / k_{B} T}
$$


By equating (2.49) and (2.46), and by integrating over $Q$ with the help of Eq. (2.47), one arrives at the following expression for the nucleation rate

$$
J(t)=\frac{D\left(Q_{0}\right)}{Q_{2}-Q_{1}} e^{-\Delta G_{0} / k_{B} T}\left(e^{\mu_{1} / k_{B} T}-e^{\mu_{2} / k_{B} T}\right),
$$

where the sub-indexes 0,1 and 2 indicate that the corresponding function is evaluated at $Q_{0}, Q_{1}$ and $Q_{2}$, respectively.

Having obtained the nucleation rate expression for the quasi-stationary case, our goal now will be to show that it is equivalent to the method of reactive flux equation derived from linear response theory. An important difference between our formalism and the method followed in the simulations is that we are working with a non-equilibrium ensemble of replicas, while in simulations one makes reference to a single system or a single replica. The connecting point that links our scheme with the results referred to a single system is the probabilistic interpretation of the ensemble number density $f(Q, t)$.

Bearing this probabilistic interpretation in mind, we will see how to recover the transition rate expression (2.81). The first step will be to transform the continuity equation into the phenomenological starting equation of the method of reactive flux. By integrating Eq. (2.8) from $Q_{1}$ to $Q_{0}$, and using the quasistationary condition (2.46) and the result (2.50) one obtains

$$
\frac{d}{d t} \int_{Q_{1}}^{Q_{0}} f(Q, t) d Q=-J(t)=\frac{D_{0}}{Q_{2}-Q_{1}} e^{-\Delta G_{0} / k_{B} T}\left(e^{\mu_{2} / k_{B} T}-e^{\mu_{1} / k_{B} T}\right),
$$

where $\int_{Q_{1}}^{Q_{0}} f(Q, t) d Q=P_{A}(t)$ is just the probability that the system would be at the left hand side of the barrier, at time $t$.

From (2.21) the diffusion coefficient expression at the top of the barrier is given by

$$
D\left(Q_{0}, t\right)=\frac{k_{B} L\left(Q_{0}\right)}{f\left(Q_{0}, t\right)}
$$

By using the expression for the chemical potential (2.7) and (2.48), the density $f\left(Q_{0}, t\right)$ could alternatively be expressed as

$$
f\left(Q_{0}, t\right)=e^{\mu_{1} / k_{B} T} e^{-\Delta G_{0} / k_{B} T} .
$$

On the other hand, from Eq. (2.48) and (2.7) it follows 


$$
e^{\mu_{2} / k_{B} T}=f_{2} e^{\Delta G_{2} / k_{B} T}=\frac{\int_{Q_{0}}^{Q_{2}} f(Q, t) d Q}{\int_{Q_{0}}^{Q_{2}} e^{-\Delta G / k_{B} T} d Q}=\frac{P_{B}}{\int_{Q_{0}}^{Q_{2}} e^{-\Delta G / k_{B} T} d Q} .
$$

Here $P_{B}$ represents the probability of being at the right hand side of the barrier. By introducing these two later expressions into Eq. (2.51), one arrives at

$$
\frac{d P_{A}(t)}{d t}=k_{B A}(t) P_{B}(t)-k_{A B}(t) P_{A}(t)
$$

where

$$
k_{A B}(t)=\frac{L\left(Q_{0}\right) k_{B}}{\left(Q_{2}-Q_{1}\right) P_{A}(t)}
$$

and

$$
k_{B A}(t)=\frac{L\left(Q_{0}\right) k_{B}}{\left(Q_{2}-Q_{1}\right) P_{A}(t)} \frac{\int_{Q_{1}}^{Q_{0}} e^{-\Delta G / k_{B} T} d Q}{\int_{Q_{0}}^{Q_{2}} e^{-\Delta G / k_{B} T} d Q}
$$

are the forward and backward rates, respectively.

Therefore, we have just obtained the equation governing the evolution of the probability that the system would be in the metastable side of the barrier. At this point, we have to highlight two important differences with respect to method of reactive flux. First, we have obtained this equation as a particular case (corresponding to a quasi-stationary situation) of a more general theory of wider applicability. Moreover, the rate coefficients in our expression are explicitly time-dependent, which does not happen in the formula of the method of reactive flux. Finally, it is important to remark, that our rate coefficients fulfill the detailed balance condition

$$
\frac{k_{A B}(t)}{k_{B A}(t)}=\frac{P_{B}^{e q}}{P_{A}^{e q}}
$$

as follows from Eqs. (2.1), (2.56) and (2.57). This condition guarantees that the flux vanishes at equilibrium.

The last task to carry out is to compare the rate $k_{A B}(t)$ with the reactive flux formula (2.81). Within that framework, the expression (2.78) is identified with the rate $k_{A B}$ at times large enough so that the correlations have achieved a stable value characterized by a plateau. In our expression, for long times the probability $P_{A}$ will approach and can be replaced by the equilibrium value $P_{A}^{e q} \Leftrightarrow\left\langle n_{A}\right\rangle_{e q}$, and therefore $k_{A B}$ will become time independent. 
On the other hand, the random component $J^{r}$ of the current appearing in the Green-Kubo expression (2.17) is related with the fluctuations of $P_{A}$ with respect to the equilibrium value. Indeed, by integrating the continuity equation (2.8) from $Q_{1}$ to $Q$ one obtains

$$
\frac{\partial N(Q, t)}{\partial t}=-J(Q, t)=-J^{s}(Q, t)-J^{r}(Q, t)
$$

where

$$
N(Q, t)=\int_{Q_{1}}^{Q} f\left(Q^{\prime}, t\right) d Q^{\prime} .
$$

Notice that Eq. (2.59) is valid for arbitrary times. In a very short time scale -microscopic time scale [17]- we can write

$$
\frac{\partial \delta N(Q, t)}{\partial t}=-J^{r}(Q, t)
$$

where the time derivative $\frac{\partial \delta N(Q, t)}{\partial t}$ is the instantaneous rate of change [42]. Therefore

$$
\left\langle J^{r}\left(Q^{\prime}, 0\right) J^{r}(Q, t)\right\rangle_{e q}=\left\langle\frac{\partial \delta N\left(Q^{\prime}, 0\right)}{\partial t} \frac{\partial \delta N(Q, t)}{\partial t}\right\rangle_{e q}
$$

being $\delta N=N-N_{e q}$. In the quasi-stationary state, described by Eq. (2.46), the quantity $\frac{\partial \delta N\left(Q^{\prime}, t\right)}{\partial t}$ is independent of the position $Q^{\prime}$ of the absorbing barrier. Hence, we can particularize for convenience the later expression for $Q^{\prime}=Q_{0}$, and taking into account that

$$
\frac{d \delta N\left(Q_{0}, t\right)}{d t}=\frac{d}{d t}\left\langle\Delta n_{A}(t)\right\rangle=-\frac{d}{d t}\left\langle\Delta n_{B}(t)\right\rangle
$$

we can then rewrite the phenomenological coefficient as

$$
k_{B} L\left(Q_{0}\right)=\left(Q_{2}-Q_{1}\right) \int_{0}^{\infty} d t\left\langle\left\langle\dot{n}_{B}(0)\right\rangle\left\langle\dot{n}_{B}(t)\right\rangle\right\rangle_{e q} .
$$

Finally, substitution of this expression in Eq. (2.56) yields

$$
k_{A B}=\frac{1}{\left\langle n_{A}\right\rangle_{e q}} \int_{0}^{\infty} d t\left\langle\left\langle\dot{n}_{B}(0)\right\rangle\left\langle\dot{n}_{B}(t)\right\rangle\right\rangle_{e q} .
$$

By remembering the link between ensemble averages and the values associated to a single system, the equivalence between this equation and expression (2.81) is then obviously proven. 


\subsubsection{General Case}

The method of reactive flux used by Frenkel and coworkers in their simulations considers that the barrier separates the system into two states, $A$ and $B$, and it is restricted to the evaluation of the transition rate between these initial and final states. In particular, it provides no information about the evolution of intermediate configurations. In the previous section, we have proved that this scheme corresponds to a quasi-stationary case associated to high nucleation barriers. The theory we propose also allows us to evaluate the evolution and the time-dependent rate of change of the global crystallinity $Q$ of the system, at any intermediate state between $Q_{1}$ and $Q_{2}$.

Our scheme is formulated in terms of a Fokker-Planck equation accounting for the evolution of $f(Q, t)$. From the knowledge of the number density $f(Q, t)$ one could then evaluate the relevant quantities of the system.

In the general case, one must work with the kinetic equation (2.20) which constitutes the central point of our approach. This equation contains the free energy barrier $\Delta G(Q)$ and the diffusion coefficient in the internal space $D(Q)$. The barrier $\Delta G(Q)$ could be evaluated from simulations analogous to the ones performed in reference [21]. In Section 2.2, we have obtained an explicit expression for the coefficient $D(Q)$. However, it is necessary to transform Eq. (2.17) into an expression more proper for simulations.

With the help of Eq. (2.61), one can relate the rate of change of the order parameter with the random contribution to the current in the following way

$$
\langle\dot{Q}(t)\rangle=\int_{Q_{1}}^{Q_{2}} Q \frac{\partial \delta f(Q, t)}{\partial t} d Q=\int_{Q_{1}}^{Q_{2}} J^{r}(Q, t) d Q .
$$

By substitution of the later expression and Eq. (2.61) into the Green-Kubo formula (2.17) for $D(q)$ (where $q$ represents an arbitrary value of the degree of crystallization), and using Eq. (2.21) we finally obtain

$$
k_{B} L(q)=-\int_{0}^{\infty} d t\langle\langle\dot{Q}(0)\rangle \delta \dot{N}(q, t)\rangle_{e q}
$$

In the equation above there appears the expected value $\langle\dot{Q}(0)\rangle$, which could be identified with the initial speed of change $\dot{Q}(0)$ of the system which is being simulated. Additionally, the term $\delta \dot{N}(q, t)$ is given by

$$
\delta \dot{N}(q, t)=-\left\langle\dot{n}_{q}(t)\right\rangle,
$$

where $n_{q}(t)=\theta[Q(t)-q]$. 
With these considerations in mind, the definitive expression in a simulable form yields

$$
L(q)=\frac{1}{k_{B}} \int_{0}^{\infty} d t\left\langle\dot{Q}(0) \dot{n}_{q}(t)\right\rangle_{e q} .
$$

Note that the later equation for the phenomenological coefficient is formally similar to expression (2.81), analyzed in Section 2.5.1 and in the Appendix. However, two significant differences exist. The first difference comes from the fact that in Eq. (2.81), the only trajectories giving a non-vanishing contribution are the ones corresponding to configurations initially at the top of the barrier. The second consists of the replacement of the characteristic function $n_{B}(t)=\theta\left[Q(t)-Q_{0}\right]$ of Eq. (2.81) for $n_{q}(t)$. Therefore, by performing simulations analogous to the ones carried out by Frenkel and coworkers, we could obtain the phenomenological coefficient $L(q)$ for any value $q$ of the degree of crystallization. Knowing $L(q)$, since the barrier $\Delta G(Q)$ could be evaluated from simulations, we could completely determine the Fokker-Planck Eq. (2.20). By solving this equation we would know the probability density $f(Q, t)$ describing the dynamics of the system.

\subsection{Conclusions}

In this chapter, we have introduced a new method based upon mesoscopic nonequilibrium thermodynamics to describe the nucleation kinetics from a mesoscopic and nonequilibrium perspective. This method allows one to easily obtain a set of general kinetic equations of the Fokker-Planck type that not only reproduce the ones which have been proposed in the literature, but they facilitate alternative descriptions of the nucleation process. In this context, we have derived a new Fokker-Planck equation in terms of an order parameter -as the one used by Frenkel et al. [22],[23] in recent simulations- and we have also introduced an enhanced hydrodynamic model of nucleation.

We have verified that the Fokker-Planck equations we have obtained apart from having the appropriate form, they are also consistent, at least in the quasistationary case. In fact, the quasi-stationary situation corresponds to low supersaturations (and slow rates), that are the typical experimentally affordable conditions.

Moreover, our treatment links theory with simulations, in the sense that we propose Fokker-Planck equations as the ones commonly used at theoretical level, but their coefficients are susceptible to be determined from simulations. Moreover, it constitutes a unifying proposal where the expressions of the linear 
response theory emerge in the quasi-stationary limit of a more general Kramerstype theory. Besides, it offers the possibility of a deeper study of the process. As we have described in the last Section, by performing simulations analogous to the ones carried out in the quasi-stationary case, one could in principle obtain a more complete information about the process. For example, one could study time-dependent nucleation and follow the evolution and the rate of change of intermediates states.

Our model facilitates the study of time-dependent nucleation and also may include potential effects of a pre-existent clusters distribution. Moreover, this new formalism we have proposed, not only reproduces and overcomes some of the main drawbacks of previous homogeneous nucleation treatments, but rather it constitutes an appropriate framework to treat nucleation processes from a wider perspective. In fact, the formalism could easily be extended to more general situations as the case of inhomogeneous nucleation in the presence of external gradients or flows, discussed in Chapter 5 . 


\section{Appendix}

Our aim in this appendix is to present a brief review of the derivation, the limitations and the scope of applicability of the method of reactive flux to evaluate reaction-rates [43]-[45]. To this purpose, let us consider a macroscopic system whose state is characterized by a reaction coordinate $Q$. This system can be in two different states, $A$ and $B$, separated by an energy barrier whose maximum is located at position $Q_{0}$. The state of the system, i.e. the side of the barrier where it is located, can be characterized by the functions

$$
\begin{aligned}
& n_{A}(t)=\theta\left[Q_{0}-Q(t)\right] \\
& n_{B}(t)=\theta\left[Q(t)-Q_{0}\right],
\end{aligned}
$$

where $\theta$ is the step function, and $n_{A}(t), n_{B}(t)$ obviously satisfy the condition

$$
n_{A}(t)+n_{B}(t)=1 \text {. }
$$

The probability that our system is at state $A$ at time $t$, will be given by the non-equilibrium average of the characteristic function $n_{A}(t)$

$$
P_{A}(t)=\left\langle n_{A}(t)\right\rangle .
$$

The starting point of this theory is to postulate the validity of the following phenomenological equation describing the dynamics of the populations at states $A$ and $B$

$$
\frac{d P_{A}(t)}{d t}=k_{B A} P_{B}(t)-k_{A B} P_{A}(t)
$$

where $P_{B}(t)=1-P_{A}(t)$, and $k_{A B}$ and $k_{B A}$ are the forward and backward rates, respectively. These rates are assumed to be constant and verify the detailed balance condition

$$
\frac{k_{A B}}{k_{B A}}=\frac{P_{B}^{e q}}{P_{A}^{e q}} .
$$

In the spirit of the Onsager's regression hypothesis, one assumes that the relaxation of $\Delta P_{A}(t) \equiv P_{A}(t)-P_{A}^{e q}$ from an initial non-equilibrium deviation $\Delta P_{A}(0)$ follows the same exponential decay as the equilibrium correlation function of the fluctuations 


$$
\frac{\Delta P_{A}(t)}{\Delta P_{A}(0)}=\frac{\left\langle\Delta n_{A}(0) \Delta n_{A}(t)\right\rangle_{e q}}{\left\langle\Delta n_{A}(0) \Delta n_{A}(0)\right\rangle_{e q}}=e^{-\lambda t}
$$

where $\Delta n_{A}(t)=n_{A}(t)-\left\langle n_{A}\right\rangle_{e q}$, the symbol $\langle\ldots\rangle_{e q}$ represents an equilibrium average and the relaxation rate $\lambda$ is given by

$$
\lambda=k_{A B}+k_{B A} .
$$

From these equations one can obtain the following formula for the transition rate, employed by ten Wolde, Ruiz-Montero and Frenkel in their simulations [22]-[23]

$$
k_{A B}=\frac{\left\langle\dot{n}_{B}(0) n_{B}(t)\right\rangle_{e q}}{\left\langle n_{A}\right\rangle_{e q}}=\frac{\left\langle\dot{Q}(0) \delta\left[Q(0)-Q_{0}\right] \theta\left[Q(t)-Q_{0}\right]\right\rangle_{e q}}{\left\langle n_{A}\right\rangle_{e q}} .
$$

This expression is not valid for very short times (because the relaxation of the system cannot be exponential at $t=0$ ). Additionally, it is also restricted to times $t$ verifying the condition $t<<1 / \lambda$, since along its derivation it is assumed that $e^{-\lambda t} \approx 1$. Moreover, we notice that although $k_{A B}$ is a time independent rate, in expression (2.78) it is equated to a correlation function depending explicitly on time. Therefore, the later equation is only correct for times long enough so that the correlations have reached a plateau value.

On the other hand, taking into account the identity

$$
n_{B}(t)-n_{B}(0)=\int_{0}^{t} d t^{\prime} \frac{d n_{B}\left(t^{\prime}\right)}{d t^{\prime}}
$$

and since the equilibrium average $\left\langle\dot{n}_{B} n_{B}(0)\right\rangle_{e q}$ vanishes, one can rewrite Eq. (2.78) as follows

$$
k_{A B}=\int_{0}^{t} \frac{\left\langle\dot{n}_{B}(0) \dot{n}_{B}\left(t^{\prime}\right)\right\rangle_{e q}}{\left\langle n_{A}\right\rangle_{e q}} d t^{\prime}
$$

As asserted before, this expression is only valid for times long enough so that the correlations have achieved a plateau. For such values of time, it is then meaningful to assume that the velocity autocorrelation function has decayed to zero, so that we can replace the upper limit of the previous integral by infinity. Therefore, the final expression for the transition rate yields

$$
k_{A B}=\frac{1}{\left\langle n_{A}\right\rangle_{e q}} \int_{0}^{\infty}\left\langle\dot{n}_{B}(0) \dot{n}_{B}(t)\right\rangle_{e q} d t=\frac{1}{\left\langle n_{A}\right\rangle_{e q}} \int_{0}^{\infty}\left\langle\dot{Q}(0) \delta\left[Q(0)-Q_{0}\right] \dot{n}_{B}(t)\right\rangle_{e q} d t .
$$


This expression coincides with the one derived from our method. 


\section{Bibliography}

[1] G. Gomila, A. Pérez-Madrid, and J.M. Rubí, Physica A 233, 208 (1996).

[2] I. Pagonabarraga, A. Pérez-Madrid, and J.M. Rubí, Physica A 237, 205 (1997).

[3] I. Pagonabarraga and J.M. Rubí, Physica A 188, 553 (1992).

[4] D. Reguera, J.M. Rubí, and A. Pérez-Madrid, Physica A 259, 10 (1998).

[5] H.A. Kramers, Physica VII, 284 (1940).

[6] P. Hänggi, P. Talkner, and M. Borkovec, Rev. Mod. Phys. 62, 251 (1990).

[7] I. Prigogine and P. Mazur, Physica XIX, 241 (1953).

[8] S.R. de Groot and P. Mazur, Non-equilibrium Thermodynamics (Dover, New York, 1984).

[9] A. Pérez-Madrid, J.M. Rubí, and P. Mazur, Physica A 212, 231 (1994).

[10] J.M. Rubí and P. Mazur, Physica A 250, 253 (1998).

[11] P. Mazur, Physica A 261, 451 (1998).

[12] J.M. Rubí and A. Pérez-Madrid, Physica A 264, 492 (1999).

[13] P. Mazur, Physica A 274, 491 (1999).

[14] J.M. Rubí and P. Mazur, Physica A 276, 477 (2000).

[15] I. Santamaría-Holek, D. Reguera, and J.M. Rubí, Phys. Rev. E 63, 051106 (2001).

[16] D. Reguera and J.M. Rubí, Phys. Rev. E 64, 061106 (2001). 
[17] L.D. Landau and E.M. Lifshitz, Course of Theoretical Physics Vol 5 (Statistical Physics Part 1) and Vol 9 (Statistical Physics Part 2) (Pergamon Press, New York, 1980).

[18] J.W. Gibbs, Scientific Papers Vol 1. Thermodynamics (Longman, New York, 1906) pp 49-54.

[19] J.H. Keenan, Thermodynamics, (Wiley, New York, 1941) p. 290.

[20] H. Reiss, Methods of Thermodynamics, (Dover, New York, 1965).

[21] J.S. van Duijneveldt and D. Frenkel, J. Chem. Phys. 96, 4655 (1992).

[22] P.R. ten Wolde, M.J. Ruiz-Montero, and D. Frenkel, J. Chem. Phys. 104, 9932 (1996) and references quoted therein.

[23] P.R. ten Wolde, M.J. Ruiz-Montero, and D. Frenkel, Faraday Discuss. 104, 93 (1996).

[24] M.J. Ruiz-Montero, D. Frenkel, and J.J. Brey, Molecular Physics 90, 925 (1997).

[25] D. Reguera, J.M. Rubí, and A. Pérez-Madrid, J. Chem. Phys. 109, 5987 (1998).

[26] G. Narsimhan and E. Ruckenstein, J. Colloid Interface Sci. 128, 549 (1989).

[27] E. Ruckenstein and B. Nowakowski, J. Colloid Interface Sci. 137, 583 (1990).

[28] B. Nowakowski and E. Ruckenstein, J. Chem. Phys. 94, 1397 (1991).

[29] B. Nowakowski and E. Ruckenstein, J. Chem. Phys. 94, 8487 (1991).

[30] J.S. Langer, Ann. Phys. 41, 108 (1967).

[31] J.S. Langer, Ann. Phys. 54, 258 (1969).

[32] J.S. Langer, Physica 73, 61 (1974).

[33] D.W. Oxtoby in Fundamentals of Inhomogeneous Fluids, p. 407, (Marcel Dekker, New York, 1992).

[34] J.W. Cahn in Critical Phenomena in Alloys, Magnets and Superconductors, p. 41, (Mc Graw-Hill, New York, 1971). 
[35] J.W. Cahn and J.E. Hilliard, J. Chem. Phys. 31, 688 (1959).

[36] J.S. Langer and L.A Turski, Phys. Rev. A 8, 3230 (1973).

[37] A. Pérez-Madrid, D. Reguera, and J.M. Rubí, cond-mat/0109249, to appear in J. Phys.: Cond. Matt.

[38] S.P. Das and G.F. Mazenko, Phys. Rev. A 34, 2265 (1986).

[39] V.A. Shneidman and P. Hänggi, Phys. Rev. E 49, 894 (1994).

[40] K.F. Kelton, Solid State Phys. 45, 75 (1991).

[41] K.F. Kelton, A.L. Greer, and C.V. Thompson, J. Chem. Phys. 79, 6261 (1983).

[42] R. Zwanzig, Annu. Rev. Phys. Chem. 16, 67 (1965).

[43] D. Chandler, J. Chem. Phys. 68, 2959 (1978).

[44] D. Chandler, Introduction to Modern Stastistical Mechanics, (Oxford University Press, 1987).

[45] C.H. Bennett, in Algorithms for Chemical Computation, edited by R.E. Christofferson (American Chemical Society, Washington. D. C., 1977). 


\section{Chapter 3}

\section{The Generalization of the Nucleation Theorem}

The "nucleation theorem" is a thermodynamic formula which establishes a relation between the energy of formation of a drop and its molecular content. Its relevance lies in the fact that it constitutes a powerful tool to analyze the experimental data on homogeneous nucleation. Assuming that the preexponential factor in the nucleation rate expression (1.26) depends only weakly on supersaturation, the nucleation theorem facilitates the knowledge of the size of the critical nucleus directly from measurements of the nucleation rate as a function of the supersaturation, and independently of any cluster model. Provided that, as we showed in Chapter 1, there is still no consensus about the most correct expression for the energy of formation or even for the proper definition of a cluster, the nucleation theorem provides a useful test of validity of the different theories. That is the reason why it has been subject of intense investigations since it was conjectured by Anisimov et al. [1] and, independently, by Kashchiev [2] in 1982.

At the same time as the nucleation theorem became a common tool to infer the properties of the critical nucleus from experimental results, its credibility was put under scrutiny. The expression of the theorem was refined and its cogency was confirmed beyond the CNT, for more sophisticated cluster models. In addition, simulation results which explored the validity of the theorem at the molecular level, found a remarkable agreement, even for tiny critical clusters. However, all the previous derivations were based on particular thermodynamic models thus raising some doubts about its generality. Moreover, it was still conceivable that such an approach would fail for very small droplets.

The mesoscopic and nonequilibrium character of nucleation acted in sup- 
port of that concerns, hiding the proper meaning and scope of the nucleation theorem. Its thermodynamic nature seemed to be in contradiction with its applicability to a non-macroscopic and unstable entity as a nucleating droplet is.

Our purpose in this chapter is precisely to shed some light about the validity and significance of the nucleation theorem. First, we shall proof from different perspectives that the so-called nucleation theorem is a general relation whose applicability extends down to the molecular level and whose scope goes beyond the context of nucleation. To stress its generality, we present a purely thermodynamic, and therefore model-independent, derivation of the theorem. This derivation clearly evidences that similar relations may be extremely useful in the study of general inhomogeneous systems. In addition, we will try to clarify the relation between the nucleation theorem and the proper definition of a physically meaningful cluster.

The chapter is distributed as follows. We begin in Section 3.1 by reviewing the previous investigations concerning the nucleation theorem. In Section 3.2 we recover the derivation of the theorem originally proposed by Hill in the context of the "small system thermodynamics". Section 3.3 is devoted to present a general thermodynamic proof of the nucleation theorem. In Sec. 3.4 we test the theorem's applicability at the molecular level by examining the nonuniform distributions caused by cavities and a simple cluster in a hard rod fluid, while in Section 3.5 we show that the nucleation theorem is valid, and related to the compressibility equation of state, for the density distribution in the "atmosphere" of a molecule in a fluid. In Section 3.6 we use the nucleation theorem to derive the Gibbs absorption equation whereas in Section 3.7 the meaning of the molecular excess is reconsidered in the context of the nucleation theorem. Finally, we present a summary of our main findings in Section 3.8.

\subsection{Introduction}

The "nucleation theorem" was proposed by Anisimov et al. [1] in 1982 in connection with classical nucleation theory. In fact, from equations (1.19) and (1.21) it is straightforward to verify that the classical work of formation of a drop is related to the number of molecules that it contains through the expression

$$
\left(\frac{\partial \Delta G_{C N T}^{*}}{\partial \Delta \mu}\right)_{V, T}=-n^{*}
$$

In the same year, Kashchiev [2] independently derived a more general expression using a phenomenological model for the work of formation of a cluster. 
Writing the work required to form a cluster of $n$ molecules as

$$
W(n, \Delta \mu)=-n \Delta \mu+F(n, \Delta \mu),
$$

where $\Delta \mu$ is the difference of chemical potentials between the metastable and the stable phase, and $F(n, \Delta \mu)$ is the excess free energy of the cluster, Kashchiev showed that

$$
\left(\frac{\partial \Delta W^{*}}{\partial \Delta \mu}\right)_{V, T}=-n^{*}+\left(\frac{\partial F^{*}}{\partial \Delta \mu}\right)_{V, T} .
$$

The importance of the nucleation theorem becomes apparent from its relation to the rate of nucleation. As we saw in Chapter 1, the steady-state nucleation rate is usually expressed in an Arrhenius form,

$$
J=K \exp \left(-\Delta W^{*} / k_{B} T\right),
$$

where $K$ is a preexponential kinetic factor. Taking the logarithm of Eq. (3.4), differentiating with respect to $\Delta \mu$, and using Eq. (3.3) yields

$$
\left(\frac{\partial\left(k_{B} T \ln J\right)}{\partial \Delta \mu}\right)_{V, T}=n^{*}+\left(\frac{\partial\left(k_{B} T \ln K\right)}{\partial \Delta \mu}\right)_{V, T}-\left(\frac{\partial F^{*}}{\partial \Delta \mu}\right)_{V, T} .
$$

Kashchiev then pointed out that if both $K$ and $F$ are essentially independent of the chemical potential we can obtain the size of the critical nucleus directly from isothermal experiments measuring the nucleation rate $J$ as a function of $\Delta \mu$ (or supersaturation). The nucleation theorem clearly provides an essential link between theory, for which $n^{*}$ can be calculated for different models, and experiment. In his work [2] Kashchiev explored this relation for nucleation in a variety of systems, including homogeneous and heterogeneous nucleation in vapors, nucleation in solutions and melts, and electrolytic nucleation.

Subsequent studies seem to have verified that the theorem is surprisingly general. For the case of nearly ideal mother phases, Strey, Wagner and their coworkers [3] have used the nucleation theorem, in connection with various of their experimental studies, to infer nucleus sizes. At the same time, working in the framework of CNT, they also used the Kelvin relation [4] to infer nucleus size [5] and found a remarkable level of agreement between the two inferences, thus rendering as credible, both the nucleation theorem and the extrapolation of the Kelvin relation down to the molecular level. Oxtoby and Kashchiev [6] calculated the properties of the nucleus, using density functional theory and, within the limit of accuracy of their calculation, were able to confirm it for the case of nucleation in nearly ideal argon vapor. More recently ten Wolde et al. [7], followed by $\mathrm{Oh}$ and Zeng [8], found agreement with the theorem in a 


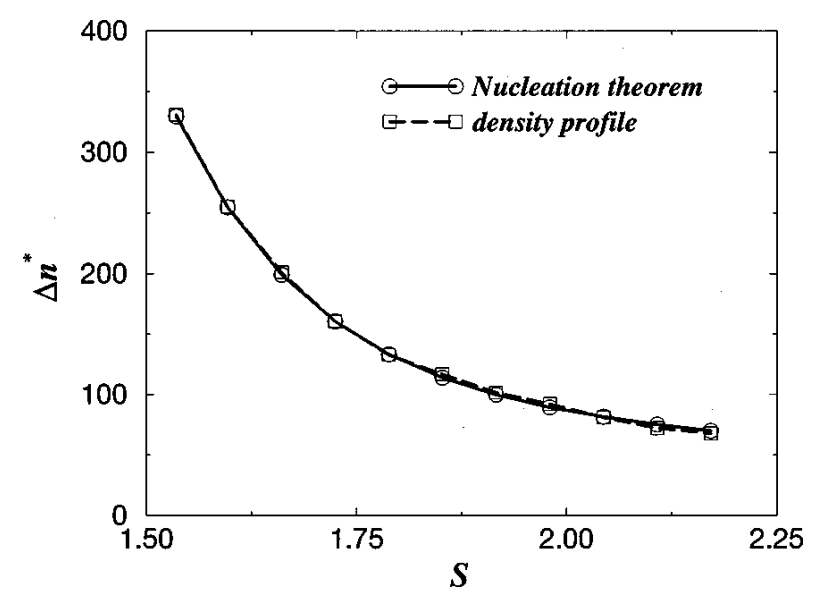

Figure 3.1: The excess number of particle $\Delta n^{*}$ in the critical cluster as a function of the supersaturation $S$ obtained from MC simulations of nucleation in argon by integrating the density profile (squares) and by using the nucleation theorem (circles). (From Ref. [7]).

Monte Carlo (MC) simulations of nucleation in argon vapor, as Fig. 3.1 shows. Kashchiev [9] and Ford [10]-[12] have made extensive use of the nucleation theorem in the analysis of experimental data. Moreover, the nucleation theorem is the basis of the scaling relations proposed by McGraw and Laaksonen [13], which have succeed in explaining the systematic discrepancies between CNT and experiments.

In 1993 Viisanen et al. [3] attempted a statistical mechanical derivation of the nucleation theorem, using the grand ensemble. Subject to the limitation that there were no interfacial effects between the system and the reservoir of constant chemical potential, these authors were able to validate a relation that differed from Eq. (3.1) in that an "excess quantity", $n^{*}-\bar{n}$, appeared in place of $n^{*}$. Roughly speaking, $\bar{n}$ represents the average number of molecules of the uniform phase that would occupy the "volume" of the nucleus. Somewhat later, in an independent analysis appearing in his thesis, ten Wolde [14] repeated the analysis of Viisanen et al., and did so, not only within the grand ensemble, but within a variety of ensembles. Again, the derivation was limited to the case where interfacial effects were absent. In these analyses the nucleation theorem proved applicable, not only to the nucleus, but to all other clusters as well.

In 1994 Oxtoby and Kashchiev [6] published a thermodynamic proof of the nucleation theorem using the Gibbsian model of a drop [15] and also extended this proof to crystal and bubble nuclei. In this work, there was no limitation equivalent to the absence of interfacial effects. At this juncture it is appropriate 
to indicate that, physically, the absence of interfacial effects implies that the cluster representing the nucleus is surrounded by an ideal gas medium. Thus the result of Oxtoby-Kashchiev, which includes interfacial effects, is applicable to nonideal as well as ideal systems. Later, Ten Wolde [14] placed this result on a somewhat sounder basis. Ford [10] also published a thermodynamic derivation of the nucleation theorem while Kashchiev has recently presented a review of the theorem [16].

In an attempt to determine how far the thermodynamic result could be extended down toward the molecular level, and in particular, whether the nucleation theorem was indeed applicable to a nonideal system, Bowles et al. [17] presented a statistical mechanical theory in which interfacial effects were included. In this theory, a term associated to the interfacial effects, admitted no clear interpretation except for the case of an ideal mother phase, in which case it vanished. Bowles et al. speculated that it would require a mathematical miracle in order for this term to yield the nucleation theorem for a nonideal mother phase, but remarked that such a miracle could be possible. Since then it has become apparent that the additional term represented an additional contribution to the excess so that it would not have to vanish, as we will explain later on.

Finally, it is imperative to mention that until now nobody realized that Hill [18],[19] had derived relations equivalent to the nucleation theorem as long ago as 1962, although they were never explicitly applied to nucleation. To credit him with having proposed the first expression of the theorem, we will devote the following section to revisit Hill's pioneering work.

\subsection{Derivation of the Nucleation Theorem from Small System Thermodynamics}

Expression (3.3) evidences the fact that the nucleation theorem is a general relation involving only thermodynamic quantities. The problem is that, in the case of nucleation, these thermodynamic quantities refers to clusters of very small size, even of molecular size. The direct application of equilibrium thermodynamics to such objects could in principle be questioned. However, one can formulate an extension of the thermodynamics principles to deal with systems of mesoscopic size. That is the idea underlying the small system thermodynamics formulated by T.L. Hill almost forty years ago.

In fact, in Refs. [18] and [19], Hill not only developed this general framework but also provided an explicit derivation of the later on called nucleation 
theorem. He accomplished that in 1962, that is 20 years before the same relation was proposed by Anisimov et al. and Kashchiev. Moreover, Hill's derivation constitutes a rigorous proof of the general validity of the nucleation theorem, not only for the particular case of a critical nucleus, but also for any kind of drop or inhomogeneity in the system.

One of our aims in this chapter is precisely to give pioneer Hill's work the credit and recognition that it deserves. To this end, we will now bring to light Hill's derivation of the nucleation theorem and try to clarify its subtleties.

The limitations of thermodynamics to deal with small systems comes from the fact that thermodynamic quantities are only properly defined for macroscopic (extensive) systems. When the system is no longer macroscopic, its properties become strongly dependent on its size. The idea proposed by Hill to overcome that difficulty is that, although the methods of classical thermodynamics cannot be applied to analyze a single small system (for instance a cluster of molecular size), they are still valid for a large (macroscopic) ensemble of those small systems. The averaged properties of this ensemble will then be representative of the behavior of a single small system.

For the sake of clarity, let us face directly the derivation of the nucleation theorem originally proposed by Hill. Consider a drop or, more in general, a density inhomogeneity in a container of volume $V$. The center of mass of the drop is restrained to the origin and it is in contact with a metastable vapor characterized by a temperature $T$, and chemical potential $\mu$. The volume $V$ includes the inhomogeneity and the metastable vapor, and must be such that the bulk metastable vapor properties $(\mu, T)$ are reached at least at the boundary of $V$; otherwise, the election of $V$ is arbitrary.

Let us construct a macroscopic system, constituted by a collection of $\mathcal{N}$ replicas of this system of volume $V$ containing inside the inhomogeneity plus surrounding vapor. For this ensemble, thermodynamics is applicable and in particular the first law yields

$$
d U=T d S-p_{0} \mathcal{N} d V+\mu d N+X d \mathcal{N}
$$

where $U, S$, and $N$ are the total energy, entropy and number of particles, respectively, $p_{0}$ is the pressure of the vapor at the periphery, and $X=\left(\frac{\partial U}{\partial \mathcal{N}}\right)_{S, V, N}$ can be regarded as the work required to introduce another replica in the system. Integration of the previous equation at constant $T, \mu$, and $V$ yields

$$
X=\bar{U}-T S-\mu \bar{N},
$$

where $\bar{U}=U / \mathcal{N}, S=S / \mathcal{N}$, and $\bar{N}=N / \mathcal{N}$ are the average energy, entropy and number of particles of the system including the drop. Notice that, $S$ does not 
need to be averaged, because the entropy has the same value for each system of the ensemble $[19,20]$.

Since we are interested in the properties solely of the drop, let us now consider a reference state constituted by the same vapor at $T, \mu$, but without any drop inside. Following the same steps than before, we can construct an ensemble of such homogeneous systems, formulate the first law

$$
d U_{0}=T d S_{0}-p_{0} \mathcal{N} d V+\mu d N_{0}+X_{0} d \mathcal{N}
$$

and obtain

$$
X_{0}=\bar{U}_{0}-T S_{0}-\mu \bar{N}_{0},
$$

where the upper bar represents average over the ensemble and the subindex 0 denotes the properties of this homogeneous vapor. Precisely from the fact of being an homogeneous system, following thermodynamics the work to introduce a new replica in the ensemble is simply the conventional volume work $X_{0}=$ $-p_{0} V$. In turn, $X$ has the meaning of the average work necessary to create a single small system containing the inhomogeneity. Thus, $W_{c} \equiv X-X_{0}$ is the work involved in the creation of the drop. Due to the subtraction of the reference state, $W_{c}$ is independent of the choice of the volume $V$. Then subtracting equations (3.7) and (3.9), and using Eqs. (3.6) and (3.8) one obtains

$$
d W_{c}=-\Delta S d T-\Delta \bar{N} d \mu,
$$

where $\Delta S=S-S_{0}$ and $\Delta \bar{N}=\bar{N}-\bar{N}_{0}$ refer to the inhomogeneity. The nucleation theorem follows as an immediate consequence of the previous equation

$$
\left(\frac{\partial W_{c}}{\partial \mu}\right)_{T}=-\Delta \bar{N}
$$

where $\Delta \bar{N}$ is the average excess of number of particles associated to the inhomogeneity. In addition, a similar relation holds for the entropy

$$
\left(\frac{\partial W_{c}}{\partial T}\right)_{\mu}=-\Delta S,
$$

where $\Delta S$ is the average entropy excess.

At this point, it is worth to emphasize some questions concerning Hill's derivation in order to clarify its generality and rigorousity. Notice that there is not restriction on the size of the inhomogeneity since using a macroscopic $(\mathcal{N} \rightarrow \infty)$ ensemble allows one to consider very small inhomogeneities (even of molecular size) with relatively large fluctuations. On the other hand, the 
derivation is general in the sense that it does not refer to any particular model nor require the concept of dividing surface which constitutes the main drawback of the previous thermodynamic derivations. Moreover, rigor demands that thermodynamic properties for such small systems should be defined in an averaged sense, resorting to the construction of the ensemble to get a true macroscopic system in which thermodynamics can safely be applied. Consequently, the molecular excess appearing in the nucleation theorem is the average excess present in the whole system and it may not be entirely confined into a particular region. These concepts will become more clear in the molecular examples developed in Sec. 3.4, and in the discussion of Sec. 3.7.

\subsection{Thermodynamic Proof of the Generalized Nucle- ation Theorem}

The content of the present section is devoted to derive the nucleation theorem within a more conventional thermodynamic framework which put emphasis on the generality and the validity of the theorem beyond the scope of nucleation.

The number of independent variables necessary to characterize a thermodynamic system is equal to the number of constraints imposed upon the system, or equivalently to the number of kinds of work (defining work broadly so as to include thermal and chemical works) that the system is set up to perform. A general way of formulating thermodynamic potentials can be based on this principle [21]. Before deriving the desired nucleation theorem, a brief explanation of this method is appropriate.

For a single component system, the fundamental equation of thermodynamics (combining first and second laws) may be written as

$$
\Delta U=\int T d S-\int p d V+\int \mu d N+\int y d Y+\int x d X \ldots,
$$

where the terms $y d Y$ and $x d X$ represent works performed on the system, with $x$ and $y$ symbolizing the intensive parameters while $Y$ and $X$ are the conjugate extensive parameters. The larger the number of works, the larger will be the number of independent variables.

It is useful to introduce the following expression

$$
\Delta \Phi=\Delta U-\int T d S+\int p d V-\int y d Y-\int \mu d N=\int x d X,
$$

where if the integral on the right were equal to zero, the second equation in Eq. (3.14) would simply give the fundamental thermodynamic equation for a 
system, in a state of equilibrium, that was capable of only volume and $Y$ work. In Eq. (3.14), $\Phi$ is not supposed to represent a thermodynamics state function. Indeed, only the full symbol $\Delta \Phi$ is intended to have meaning, and it simply represents the collection of terms on its right.

The system can be reversibly displaced from its state of stable equilibrium by the application of a constraint that can exchange work with the system. In Eq. (3.14), $x d X$ can represent that work. Of course, the system on the path of displacement, will have an additional independent variable corresponding to the constraint. In our example, it could be either $x$ or $X$. For any displacement of the system from a state of stable equilibrium, the second law establishes that

$$
\Delta \Phi \geq 0
$$

where the equality occurs for a reversible process and then $\Delta \Phi$ resembles the concept of minimum work [22].

It is convenient to write down Eq. (3.14) for an infinitesimal variation

$$
D \Phi=d U-T d S+p d V-y d Y-\mu d N \geq 0,
$$

where we emphasize that $D \Phi$ is not an exact differential by using $D$ instead of $d$. Using Eq. (3.16), one can now propose an arbitrary number of thermodynamic potentials characterized by the fact that their values increase along selected reversible paths of displacement from the initial state of equilibrium [21]. As an example, suppose the system is not set up to do $Y$ work as in Eq. (3.16). Then

$$
D \Phi=d U-T d S+p d V-\mu d N \geq 0 .
$$

The strategy for proposing functions of state that can serve as thermodynamic potentials is then to form a function and choose a "path" over which its differential imitates $D \Phi$ on the same path. For example, if we select a path defined as having $S, V, N$ constant then, from Eq. (3.17) we find

$$
(d U)_{S, V, N}=(D \Phi)_{S, V, N} \geq 0,
$$

so that along this path $d U$ does indeed imitate $D \Phi$, and $U$ is the required thermodynamic potential. Alternatively, if we selected a path of constant $N, P, T$, then the Gibbs free energy,

$$
G=U-T S+p V
$$

is the thermodynamic potential we must use since

$$
(d G)_{N, P, T}=(d U)_{N, P, T}-T(d S)_{N, P, T}+p(d V)_{N, P, T}=(D \Phi)_{N, P, T} \geq 0,
$$


so $d G$ imitates $D \Phi$ along this path.

We continue by considering an open system contained in a macroscopic volume $V$ which can exchange molecules with its surroundings. For simplicity we will restrict ourselves to a single component system, although multicomponent systems and other phases can be treated by the same method. The volume $V$ is chosen so that the intensive properties of the system at the boundaries are identical to the ones of the uniform system outside $V$, i.e. the nonuniformity of density lie far from the boundaries.

We introduce this restriction, because we do not want to consider a system that can exchange surface work with its surroundings, i.e. we do not want to allow $V$ to have a real physical interface with its surroundings. However, we should mention at the outset that the nucleation theorem can be derived without much additional difficulty for the case where there is a real physical interface. The restriction to nonuniformities that lie far from the boundary will not compromise our goal, since in the end we will be interested in mesoscopic or molecular non uniformities that can easily be positioned far from the boundaries of a macroscopic containing volume.

We now refer to $D \Phi$ defined in Eq. (3.16) in order to construct a particular thermodynamic potential for a system capable of performing both volume and $Y$ work. In this case, we are interested in examining systems which are inhomogeneous so that $y$ is associated with the field required to create such an inhomogeneity. This field may take on a variety of forms, including a wall, an electric field, or it may be some more complicated field that can form a small cluster or drop. It is important to note that we need not specify exactly what the field is and that we can select fields to reproduce molecular sized inhomogeneities. Consequently, although we are using the tools of macroscopic thermodynamics, our proof is valid even for these molecular sized inhomogeneities as long as we deal with the average inhomogeneity under the constraint.

Consider using $y$ to displace an initially homogeneous system, capable only of volume work, along a path of constant $\mu, V, T$ so that the system along this path is nonuniform. If we introduce the usual grand potential,

$$
\Omega=U-T S-\mu N
$$

then it is straightforward to show that $d \Omega$ will imitate $D \Phi$ of Eq. (3.17) along a path of constant $\mu, V, T$. Thus $\Omega$ is the appropriate thermodynamic potential along such a path. Since $y$ is displacing the system along this path, it performs the reversible work, $y d Y=D W_{c}$ on the system and the fundamental equation of thermodynamics becomes 


$$
d U=T d S-p d V+y d Y+\mu d N
$$

or

$$
\begin{aligned}
& (d U)_{\mu, V, T}-T(d S)_{\mu, V, T}-\mu(d N)_{\mu, V, T}=\quad(d \Omega)_{\mu, V, T} \\
& =y(d Y)_{\mu, V, T}=\left(D W_{c}\right)_{\mu, V, T},
\end{aligned}
$$

where we use $D$ to emphasize that work is not an exact differential, i.e., we cannot say that the system contains a certain amount of "work".

Note that $\Omega$ becomes a function of $Y$ as well as of $\mu, V, T$ along the path of displacement. Also, integration of Eq. (3.23) gives

$$
\Omega(\mu, V, T, Y)-\Omega_{0}\left(\mu, V, T ; Y_{0}(\mu, V, T)\right)=\left(W_{c}\right)_{\mu, V, T}
$$

where we have used the $\Omega_{0}$ to denote the value of $\Omega$ in the initial equilibrium state in which the system is set up in a manner that allows it to perform only volume work. In that state, there are only three independent variables which we have chosen to be $\mu, V, T$, so that $Y$ in that state, denoted by $Y_{0}$, is itself a function of $\mu, V, T$ and is fully determined when these variables are fixed. The semicolon in Eq. (3.24) emphasizes this special character of $Y_{0}$. Performing a Legendre transformation of $\Omega$ in Eq. (3.21) it yields

$$
d \Omega=-S d T-p d V-N d \mu+y d Y
$$

From this relation it follows that

$$
N=-\left(\frac{\partial \Omega}{\partial \mu}\right)_{V, T, Y}
$$

and

$$
S=-\left(\frac{\partial \Omega}{\partial T}\right)_{V, \mu, Y} .
$$

We now wish to determine how $\left(W_{c}\right)_{\mu, V, T}$ changes when $\mu$ is changed. A common practice is to refer to the derivative $\partial W_{c} / \partial \mu$. However, this is inaccurate since $D W_{c}$ is not a complete differential and does not have a well defined derivative. Instead, we must consider the "ratio" $\left(D W_{c} / \partial \mu\right)$ at constant $V, T$, $Y$. 
Returning to Eq. (3.24), we can write

$$
\left(\frac{D W_{c}}{\partial \mu}\right)_{V, T, Y}=\left(\frac{\partial \Omega}{\partial \mu}\right)_{V, T, Y}-\left(\frac{\partial \Omega_{0}}{\partial \mu}\right)_{V, T}
$$

where, since $\Omega_{0}$ depends only on $\mu, V, T$ and not on a fourth independent variable $Y_{0}$, the derivative $\Omega_{0}$ needs no $Y$ subscript. If we now substitute Eq. (3.26) into Eq. (3.28), applying the relation, respectively, to the initial uniform state and to the final nonuniform one (produced by applying the field $y$ ) we obtain

$$
\left(\frac{D W_{c}}{\partial \mu}\right)_{V, T, Y}=-\left(N-N_{0}\right)=-\Delta N
$$

where $\Delta N$ is the excess number of molecules. An exactly similar procedure, utilizing Eq. (3.27), leads to

$$
\left(\frac{D W_{c}}{\partial T}\right)_{V, \mu, Y}=-\left(S-S_{0}\right)=-\Delta S,
$$

where $\Delta S$ is the excess entropy. Equations (3.29) and (3.30) constitute the nucleation theorem. Note that $Y$ (or $y$ ) is always adjusted to be consistent with the external field that produces the final nonuniformity. Furthermore, Eqs. (3.29) and (3.30) are more general than the nucleation theorem derived with the "nucleus" of nucleation theory in mind, since they can be applied to any system in which an equilibrium non uniform density is induced by an external field. Also note that $\Delta \mu$ in Eqs. (3.1), (3.3), and (3.5) differs from $\mu$ in the equations of this and following sections by a constant. Finally, note that we also find the analogous relation for the pressure

$$
\left(\frac{D W_{c}}{\partial V}\right)_{T, \mu, Y}=-\left(p-p_{0}\right)=-\Delta p
$$

where $\Delta p$ is the excess pressure.

All these relations are almost intuitively obvious. For example, the "chemical work" involved in moving "excess" molecules from the reservoir into $V$, at a maintained chemical potential, should be $W=-\mu \Delta N$, and the derivative of this equation with respect to $\mu$ should be Eq. (3.29). However, rigor demands that we follow the quantitative thermodynamic protocol presented above. It should be reemphasized that Eqs. (3.29) and (3.30) are those derived by Hill in 1962 by another method [18], reviewed in the preceding section.

Although our analysis has been focused on a single component system, a simple extension of the argument to multicomponent systems easily yields the 
relations

$$
\begin{gathered}
\left(\frac{D W(\{\mu\})}{\partial \mu_{i}}\right)_{T, V, \mu_{j} \neq \mu_{i}}=- \text { excess of component } i \\
\left(\frac{D W(\{\mu\})}{\partial T}\right)_{\{\mu\}, V}=- \text { excess of entropy }
\end{gathered}
$$

where $\{\mu\}$ is the set of all chemical potentials.

The great generality of the theorem formulated in terms of thermodynamic relations (Eqs. (3.29) and (3.30)) makes it possible its application to different situations. One possible model is the drop, mentioned above, in equilibrium with its vapor. This of course is the original system to which the nucleation theorem was applied. However, even a concrete model as this one admits different possibilities. For example we could choose the capillarity model of classical nucleation theory, or we could choose the more sophisticated Gibbs model treated by Oxtoby and Kashchiev [6]. In particular, we would have to specify (at least implicitly) the field responsible for the local nonuniformity in density, and this also becomes part of the model. In each case, the model is defined, in part, by the number of independent variables that must be used to describe it. When we pass from the capillarity model to the Gibbs model, curvature appears as an additional variable, and if we elect to consider drops only slightly larger than molecules, other variables might become important.

As a corollary, in choosing a cluster model, e.g. the Stillinger cluster discussed in Section 1.2.5, to be used in a simulative test of the nucleation theorem, one must be careful to include the part of the "excess" that lies outside of the cluster, as will be explained in Sec. 3.7. This is not a serious problem where the surrounding phase is almost ideal (as in the simulation of ten Wolde [7]), but it grows in importance as the system becomes increasingly nonideal.

The argument which might be raised, however, is that the nucleation theorem applies to a thermodynamically metastable or unstable system containing the nucleus. The answer to this claim is that, in order to calculate the work of formation of this nucleus, it must be implicitly stabilized by a constraint, e.g. an external field, that converts it to a truly stable equilibrium to which thermodynamics can be applied. Indeed, it is this constraint that performs the reversible work required in the formation of the nucleus.

Note that, as far as the theorem represented by Eqs. (3.29) and (3.30) is concerned, there is nothing in its proof that prevents it from applying right down to the molecular level, provided that the various thermodynamic quantities are interpreted as time or ensemble averages in the presence of the field that assures stable equilibrium. 
In the remainder of this chapter we shall study a number of systems other than that involving a nucleus. These can be of molecular size or larger, and we will show, by means of statistical mechanics, that the nucleation theorem is exact as far as these systems are concerned. In this way, the proof presented above is further confirmed for those systems. This analysis is initiated in the following section.

\subsection{Nonuniform Density Distributions in a 1-D Hard Rod Fluid}

The nucleation theorem has been proved to be of general applicability to equilibrium systems containing nonuniform distributions of density and is valid down to the molecular level. To show this assertion, we will study several systems that can be analyzed exactly using statistical mechanics. First, we will examine two nonuniform density distributions formed in a one dimensional hard rod fluid, where we are able to analytically calculate both the work of generate the uniformity and the excess number of particles. The density distributions we will consider, respectively, are that which results from the presence of a cavity and that due to the presence of a simple cluster.

Our strategy will be as follows: The reversible work required to apply a constraint (or field) in order to create a nonuniform distribution can be expressed as

$$
W_{c}=-k T \ln \frac{Q_{c}}{Q_{o}}
$$

where $Q_{o}$ is the partition function of the original (uniform) phase and $Q_{c}$ is the partition function of the system subject to the constraint. Having calculated these partition functions to obtain $W_{c}$, we take the derivative of this work with respect to the chemical potential of the original phase. Application of the nucleation theorem relates this quantity with $-\Delta n$, the negative value of the excess number of particles. We then obtain the excess, via a means independent of the theorem, and compare the two quantities.

We first calculate $Q_{o}$ along with the chemical potential of the uniform state. Then we calculate both $Q_{c}$ and the excesses for both the cavity and the cluster.

\subsubsection{Initial Uniform State}

The initial uniform state for both cases consists of $N$ hard rods of size $\sigma$ on a circular ring of length $L$, where the effect of converting a straight line to a ring simply introduces periodic boundaries. To calculate the partition function $Q_{o}$, 
particle 1 is initially held fixed at $L=0$, while the remaining $N-1$ can be integrated in the same manner as described by Tonks [23] for a system of hard rods on a line without a periodic boundary. Then the final particle is integrated over the entire length $L$, to obtain

$$
\begin{aligned}
Q_{o} & =\int_{0}^{L} d x_{1} \int_{\sigma}^{x_{3}-\sigma} d x_{2} \int_{2 \sigma}^{x_{4}-\sigma} d x_{3} \cdots \int_{(N-1) \sigma}^{L-\sigma} d x_{N} \\
& =\frac{(L-N \sigma)^{N-1} L}{N !}
\end{aligned}
$$

Eq. (3.35) differs slightly from the partition function derived by Tonks due to the absence of the wall effects. The two walls in the original calculation exclude a total volume of $\sigma$ to the centers of the $N$ rods. However, as one expects, the thermodynamic quantities of the two models become the same in the thermodynamic limit because end effects become unimportant. Hence the chemical potential obtained from $Q_{o}$, is

$$
\begin{aligned}
\mu & =-k T \ln \frac{Q_{o}^{+}}{Q_{o}} \\
& =k T \ln \frac{N+1}{L}-k T \ln (1-\rho \sigma)+\frac{k T \rho \sigma}{(1-\rho \sigma)} \\
& =k T \ln \rho-k T \ln (1-\rho \sigma)+\frac{k T \rho \sigma}{(1-\rho \sigma)},
\end{aligned}
$$

where $Q_{o}^{+}$is the partition function $Q_{o}$ for $N+1$ particles and $\rho=N / L$ is the density. Eq. (3.36) is identical to the usual expression for the chemical potential of a hard rod system in the thermodynamic limit where $N+1 \approx N$. Similarly, the pressure of a hard rod system on a ring approaches that of the hard rods on line, in the limit $L \rightarrow \infty$,

$$
p=k T \frac{\partial \ln Q_{o}}{\partial L}=\frac{k T \rho}{(1-\rho \sigma)}+\frac{k T}{L} \frac{1}{(1-\rho \sigma)}=\frac{k T \rho}{(1-\rho \sigma)} .
$$

\subsubsection{Cavity Formation}

A cavity [24] excludes the centers of the rods in the fluid from a localized region, creating a local decrease in the density. If the size of the cavity, which we will denote by $\lambda$, is less than the diameter of a rod then, a rod on one side of the cavity can interact with (collide with) a rod on the other side, as depicted in Figure 3.2(a). If $\lambda \geq \sigma$, the rods cannot interact across the cavity and in effect, 


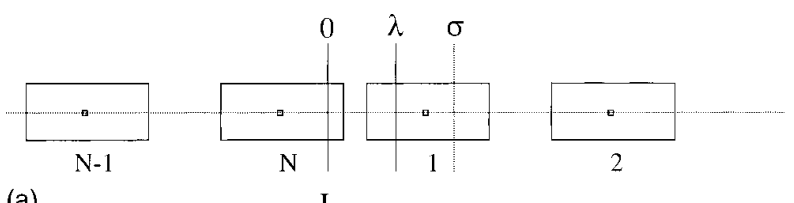

(a)

L

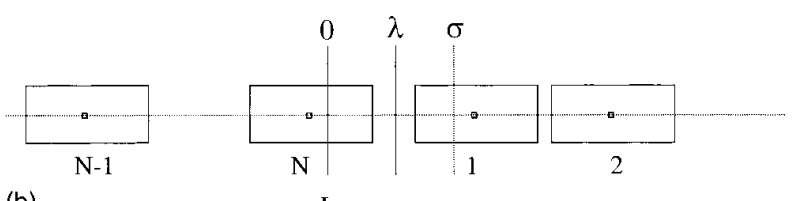

(b)

$\mathrm{L}$

Figure 3.2: The partition function for a system of hard rods of diameter $\sigma$ that contains a small cavity of length $\lambda<\sigma$ is divided into two sets of configurations. (a) Configurations in which a particle is in the region between $\lambda$ and $\sigma$ so that particle 1 interacts with particle $N$ across the cavity. These contribute to $Q_{c}^{1}$. (b) Configurations in which no particle is in the region between $\lambda$ and $\sigma$ and there is no possibility of interaction. These contribute to $Q_{c}^{2}$. Large cavities of length $\lambda \geq \sigma$ only have configurations of this type.

the rods "see" a hard wall. To calculate the work of forming a cavity of any size we treat these two cases independently.

Starting with the small cavity $(\lambda<\sigma)$ we place the center of the cavity at the point $\lambda / 2$ so that the centers of the rods are excluded from the region between 0 and $\lambda$. We then note that the full partition function for this system can be divided into two sets of configurations: those configurations, $Q_{c}^{1}$, in which a rod is in the region between $\lambda$ and $\sigma$ so that it can interact across the cavity (Fig. $3.2 \mathrm{a}$ ), and those configurations $Q_{c}^{2}$, in which there is no rod in this region so that there is no interaction (Fig. 3.2b). It is obvious for a system containing a large cavity $(\lambda \geq \sigma)$ that the full partition function will be related to configurations similar to $Q_{c}^{2}$, except that there will be no rods in a region between 0 and $\lambda$.

To obtain $Q_{c}^{1}$, we begin by holding the first rod fixed at a point $d x_{1}$ between $\lambda$ and $\sigma$ and noting that the $N$ th rod on the line will interact with this fixed rod before reaching the wall of the cavity at $L$. The upper limit of integration for the $N$ th rod will be $L-\sigma+x_{1}$, so we have to integrate over the $N-1$ particle as usual, and then integrate the first particle over the region between $\lambda$ and $\sigma$, which gives

$$
\begin{aligned}
Q_{c}^{1} & =\int_{\lambda}^{\sigma} d x_{1} \int_{x_{1}+\sigma}^{x_{3}-\sigma} d x_{2} \int_{x_{1}+2 \sigma}^{x_{4}-\sigma} d x_{3} \cdots \int_{x_{1}+(N-1) \sigma}^{L-\sigma+x_{1}} d x_{N} \\
& =\frac{(L-N \sigma)^{N-1}(\sigma-\lambda)}{(N-1) !} .
\end{aligned}
$$


If $x_{1}$ is greater than $\sigma$ the upper limit for the position of the $N$ th particle is no longer dependent on $x_{1}$, and simply becomes $L$ while the upper limit for the first particle becomes $L-(N-1) \sigma$. Proceeding as before, the remainder of the partition function is given by

$$
\begin{aligned}
Q_{c}^{2} & =\int_{\sigma}^{L-(N-1) \sigma} d x_{1} \int_{x_{1}+\sigma}^{x_{3}-\sigma} d x_{2} \int_{x_{1}+2 \sigma}^{x_{4}-\sigma} d x_{3} \cdots \int_{x_{1}+(N-1) \sigma}^{L} d x_{N} \\
& =\frac{(L-N \sigma)^{N}}{(N) !}
\end{aligned}
$$

which, is in fact just the Tonks partition function for a hard rod system. This is not surprising since, as we mentioned earlier, the presence of a cavity of size $\lambda=\sigma$ is equivalent to a hard wall. The full partition function for a one dimensional system of hard rods containing a cavity of size $\lambda<\sigma$ is then given by

$$
Q_{c}=Q_{c}^{1}+Q_{c}^{2}=\frac{(L-N \lambda)(L-N \sigma)^{N-1}}{N !},
$$

so that the work required to form such a cavity, obtained from Eq. (3.34), is

$$
W_{c}=-k T \ln (1-\rho \lambda) .
$$

Eq. (3.41) has been previously derived using scaled particle theory [25]. To arrive at an expression for the excess using the nucleation theorem we must take the derivative of this work with respect to the chemical potential given by Eq. (3.36). Taking the derivatives of both Eqs. (3.41) and (3.36) with respect to $\rho$, at constant $L$, and using the chain rule gives

$$
\left(\frac{\partial W_{c}}{\partial \mu}\right)=\left(\frac{\partial W_{c}}{\partial \rho}\right)_{L}\left(\frac{\partial \rho}{\partial \mu}\right)_{L}=\frac{\rho \lambda(1-\rho \sigma)^{2}}{(1-\rho \lambda)} .
$$

The total excess, $\Delta n$ can be obtained by calculating the excess for each set of configurations, $\Delta n_{1}$ and $\Delta n_{2}$, and weighting them by their respective probabilities. To obtain these excess quantities we will make use of the compressibility equation of state [20], which in one dimension can be written as

$$
\rho \int_{0}^{\infty} 2[g(r)-1] d r=\rho k T \kappa-1,
$$

where $g(r)$ and $\kappa$ are the pair correlation function and compressibility of the fluid, respectively. The connection between Eq. (3.43) and the excess becomes immediately obvious when we note that $\rho g(r)=\rho(r)$ is the local density at a 
point a distance $r$ from the center of a rod, so that expanding $\rho$ through the integral gives,

$$
\int_{0}^{\infty} 2[\rho(r)-\rho] d r=\rho k T \kappa-1,
$$

where the term in the brackets is the local excess at a point $r$. Completing the integration over $r$ then gives the total excess in the system due to the presence of a rod. We elaborate this in more detail in Section 3.5.

Coming back to the calculation of $\Delta n_{1}$ we see that the excess surrounding the fixed rod is given exactly by Eq. (3.43). However, this rod also contributes to the excess of the cavity, so to obtain $\Delta n_{1}$ we can simply add one unity to Eq. (3.43), giving

$$
\begin{aligned}
\Delta n_{1} & =\rho k T \kappa \\
& =(1-\rho \sigma)^{2},
\end{aligned}
$$

where, in the final step of Eq. (3.45), the compressibility, $\kappa=-(1 / L)(\partial L / \partial P)$, was obtained using Eq. (3.37) for the pressure.

In calculating $\Delta n_{2}$ we will make use of the fact that if the rods are unable to interact with rods on the other side of the cavity i.e. $\lambda \geq \sigma$, they are unable to distinguish between a cavity, a hard wall or another rod, so that the pair correlation function outside of such a cavity is identical to that outside of a rod. From this we can see that the excess due to a cavity of size $\lambda=2 \sigma$, so it excludes exactly the same amount of volume as another rod, is given by Eq. (3.43). The excess due to cavities of different sizes, as long as $\lambda \geq \sigma$ only differ by the number of rods or local density excluded. This is because, under this condition, at a given density the pair correlation function outside a rod or cavity depend on the size of the cavity, while the density inside a rod or cavity is zero, representing a negative contribution to the excess which depends on the excluded length. Figure 3.3 shows the density profile in a hard rod fluid due to the presence of a cavity. In general, the excess due to a cavity of length $\lambda$ is

$$
\begin{aligned}
\Delta n & =\rho k T \kappa-1-\rho(\lambda-2 \sigma): \lambda \geq \sigma \\
& =\rho^{2} \sigma^{2}-\lambda \rho .
\end{aligned}
$$

Since the configurations belonging to $Q_{c}^{2}$ have no rods in the region between 0 and $\sigma$, a situation equivalent to the presence of a cavity of size $\sigma$, we find from Eq. (3.46),

$$
\Delta n_{2}=\rho^{2} \sigma^{2}-\rho \sigma .
$$


The total excess $\Delta n$ is finally obtained by weighting $\Delta n_{1}$ and $\Delta n_{2}$ by their respective probabilities. Thus,

$$
\Delta n=\frac{Q_{c}^{1} \Delta n_{1}+Q_{c}^{2} \Delta n_{2}}{Q_{c}}=-\frac{\rho \lambda(1-\rho \sigma)^{2}}{(1-\rho \lambda)} .
$$

Comparison of Eq. (3.48) with Eq. (3.42) yields

$$
\left(\frac{\partial W_{c}}{\partial \mu}\right)=-\Delta n
$$

which is the nucleation theorem valid also for a nonuniform distribution caused by a cavity of size $\lambda<\sigma$. To complete the proof for cavities sizes $\lambda>\sigma$, it is necessary only to consider configurations of the type described by $Q_{c}^{2}$ since there is no possibility that particles interact across the cavity. $Q_{c}$ for this case can be obtained directly from Eq. (3.39) by replacing the lower limit of the integral for the particle in $d x_{1}$ with $\lambda$, thus

$$
\begin{aligned}
Q_{c}^{2} & =\int_{\lambda}^{L-(N-1) \sigma} d x_{1} \int_{x_{1}+\sigma}^{x_{3}-\sigma} d x_{2} \int_{x_{1}+2 \sigma}^{x_{4}-\sigma} d x_{3} \cdots \int_{x_{1}+(N-1) \sigma}^{L} d x_{N} \\
& =\frac{(L-N \sigma-(\lambda-\sigma))^{N}}{(N) !}
\end{aligned}
$$

and

$$
W_{c}=-k T \ln (1-\rho \sigma)-\frac{k T \rho(\lambda-\sigma)}{1-\rho \sigma} .
$$

This expression gives

$$
\left(\frac{\partial W_{c}}{\partial \mu}\right)=-\left(\rho^{2} \sigma^{2}-\lambda \rho\right)=-\Delta n
$$

which is again the nucleation theorem. The last equality on the right of Eq. (3.52) arises from the expression obtained for the excess in Eq. (3.46).

\subsubsection{Cluster Formation}

To examine a case involving a cluster we define and make use of a simple cluster consisting of two hard rods confined to a cell of length $\lambda$ fixed at a point on the ring. To ensure that the remaining $N_{2}=N-2$ rods, which constitute the surrounding phase, interact with the cluster, they are not restricted to remain outside the cell and so may penetrate the cell walls. One of such configurations is shown in Fig. 3.4. However, the particles from the surrounding phase are not 


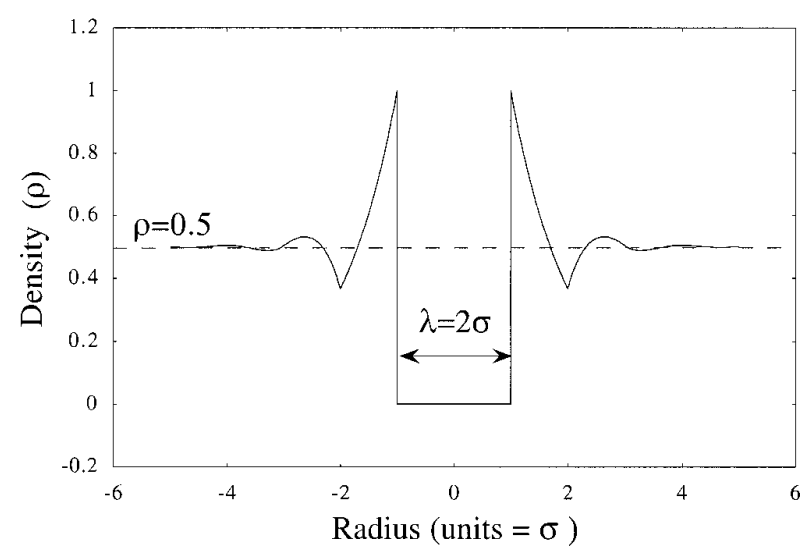

Figure 3.3: The radial density profile of the $1 \mathrm{D}$ hard rod fluid surrounding a cavity of size $\lambda=2 \sigma$ (solid line). The dashed line shows the uniform density of the fluid before the cavity was formed.

able to enter the space between the two cluster particles. In constructing the full partition function for the system, i.e. the cluster and its surroundings, it is useful to analyze Fig. 3.4. For a fixed value of $y$, which denotes the distance between the centers of the two cluster particles, the $N_{2}$ remaining particles are restricted to a region $L-y-2 \sigma$, where the $2 \sigma$ arises from the presence of the cluster particles. The position of particle 1 of the cluster can range between 0 and $\lambda-y$ while the separation between the particle 1 and particle 2 can vary from $\sigma$ to $\lambda$. The full partition function can be written as

$$
\begin{aligned}
Q_{c} & =\int_{\sigma}^{\lambda} d y \int_{0}^{\lambda-y} d x_{1} \int_{y+\sigma}^{x_{4}-\sigma} d x_{3} \cdots \int_{y+N_{2} \sigma}^{L-\sigma} d x_{N} \\
& =\frac{(L-N \sigma)^{N-2}}{(N-2) !} \int_{\sigma}^{\lambda} d y \int_{0}^{\lambda-y} d x_{1}\left(1-\frac{y-\sigma}{L-N \sigma}\right)^{N-2},
\end{aligned}
$$

where we have simply used $N_{2}=N-2$ and removed a constant from the integrand. Assuming that $L$ and $N$ are arbitrarily large so that the fraction $(y-\sigma) /(L-N \sigma)$ is arbitrarily small, the integrand in Eq. (3.53) can be expressed as an exponential. Then completing the integration over $d x_{1}$ and $d y$ we arrive at

$$
Q_{c}=\frac{(L-N \sigma)^{N}}{(N-2) !(N-2)^{2}}\left[e^{\frac{(N-2)(\lambda-\sigma)}{L-N \sigma)}}+\frac{(N-2)(\lambda-\sigma)}{(L-N \sigma)}-1\right]
$$

Using Eqs. (3.54) and (3.35) in Eq. (3.34), and assuming that in the thermodynamic limit $N-1$ and $N-2 \approx N$, yields the relation for the reversible work 


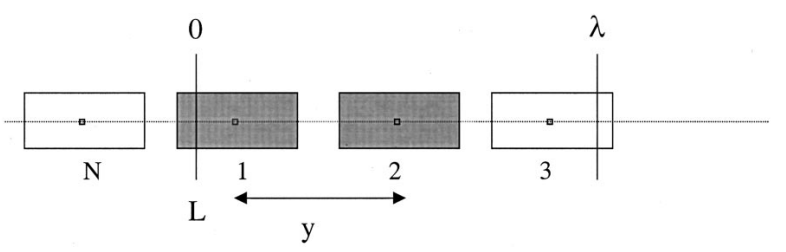

Figure 3.4: The centers of two rods belonging to the cluster (shaded) are confined a cell of length $\lambda$ while surrounding rods are able to penetrate the cell as shown. When the cluster rods are separated by a distance $y$ they create a cavity of $y+2 \sigma$.

required to form the cluster

$$
W_{c}=-k T \ln (1-\rho \sigma)-k T \ln \left[e^{\frac{(\rho(\lambda-\sigma)}{1-\rho \sigma}}+\frac{\rho(\lambda-\sigma)}{(1-\rho \sigma)}-1\right] .
$$

Finally, taking the derivative of this work with respect to the chemical potential, results in

$$
\left(\frac{\partial W_{c}}{\partial \mu}\right)=\rho \sigma(1-\rho \sigma)+\frac{\left(e^{\frac{(\rho(\lambda-\sigma)}{1-\rho \sigma}}-1\right)(\lambda-\sigma) \rho}{\left[e^{\frac{(\rho(\lambda-\sigma)}{1-\rho \sigma}}+\frac{\rho(\lambda-\sigma)}{(1-\rho \sigma}-1\right]} .
$$

To calculate the excess, we note that the two rods in the cluster separated by a distance $y$, create a cavity of size $y+2 \sigma$, and that the excess due to such a cavity is given by Eq. (3.46). However, this is not the total excess since we must add a factor 2 to account for the 2 particles inside the cluster. Thus, the excess for the cluster, when the cluster rods are separated $y$, is

$$
\Delta n(y)=\rho k T \kappa+1-\rho y .
$$

The total excess can then be expressed as a weighted average

$$
\Delta n=\int_{\sigma}^{\lambda} \Delta n(y) \Phi(y) d y
$$

where

$$
\Phi(y)=\frac{\int_{0}^{\lambda-y} d x_{1} \int_{y+\sigma}^{x_{4}-\sigma} d x_{3} \cdots \int_{y+N_{2} \sigma}^{L-\sigma} d x_{N}}{Q_{c}},
$$

is the probability density for the cluster particles to be a distance $y$ apart. Using Eq. (3.54) in Eq. (3.59) yields, after some manipulation,

$$
\Delta n=-\left[\rho \sigma(1-\rho \sigma)+\frac{\left(e^{\frac{(\rho(\lambda-\sigma)}{1-\rho \sigma}}-1\right)(\lambda-\sigma) \rho}{\left[e^{\frac{(\rho(\lambda-\sigma)}{1-\rho \sigma}}+\frac{\rho(\lambda-\sigma)}{(1-\rho \sigma}-1\right]}\right]
$$


and again we find, through comparison of this result with the right side of Eq. (3.56), that the nucleation theorem holds.

\subsection{The Nucleation Theorem and the Compressibility Equation of State}

In the previous section we have shown that the nucleation theorem is valid for any size cavity inserted into a one dimensional hard rod fluid. Furthermore, we also noted that the compressibility equation of state, Eq. (3.43), was equal to the excess due to the presence of a single rod. If we now consider the work required to insert a cavity of length $2 \sigma$, using Eq. (3.46) and the nucleation theorem, we find

$$
\left(\frac{\partial W_{c}}{\partial \mu}\right)=-(\rho k T \kappa-1)
$$

Due to the nature of the hardcore interaction between particles, inserting such a cavity into a hard rod fluid is equivalent to inserting another rod with the difference that this new "rod" has no translation [24]. We will now show that Eq. (3.61) is more generally applicable, and not restricted to a hard rod fluid.

We now turn our attention to a 3D single component system consisting of any kind of molecule and consider a field that produces a density distribution identical to that which would be produced by a molecule in the surrounding fluid fixed at a single point. In effect the field gives rise to a pseudomolecule fixed at a point. We might call this pseudomolecule an "equivalent molecule" but it is unable to move. If it was allowed to diffuse throughout the entire volume we would have introduced a new molecule to the system, and the reversible work to do this would equal the chemical potential. Thus, the reversible work required to introduce it at a fixed point is given by

$$
W=\mu-k T \ln \frac{\Lambda^{3} N}{V},
$$

where the translational component of the work has been subtracted from the full chemical potential. Taking the derivative of Eq. (3.62) with respect to $\mu$ yields

$$
\left(\frac{\partial W}{\partial \mu}\right)_{V, T}=1-\frac{k T}{N}\left(\frac{\partial N}{\partial \mu}\right)_{V, T}
$$

Writing

$$
\left(\frac{\partial N}{\partial \mu}\right)_{V, T}=\left(\frac{\partial N}{\partial V}\right)_{\mu, T}\left(\frac{\partial V}{\partial \mu}\right)_{N, T}
$$


and using the Gibbs-Duhem relation, $\partial \mu=(V / N) \partial P$, gives

$$
\left(\frac{\partial N}{\partial \mu}\right)_{V, T}=\left(\frac{\partial N}{\partial V}\right)_{\mu, T} \frac{N}{V}\left(\frac{\partial V}{\partial P}\right)_{N, T}=\rho N \kappa,
$$

where

$$
\kappa=-\frac{1}{V}\left(\frac{\partial V}{\partial P}\right)_{N, T}
$$

is the isothermal compressibility and we noted that, at constant $\mu$ and $T$, $(\partial N / \partial V)=\rho$. Substitution of Eq. (3.65) into Eq. (3.63) yields

$$
\left(\frac{\partial W}{\partial \mu}\right)_{V, T}=-(\rho k T \kappa-1) \text {. }
$$

The compressibility equation of state

$$
\Delta n=\rho \int_{0}^{\infty}[g(r)-1] 4 \pi r^{2} d r=\rho k T \kappa-1,
$$

relates $\kappa$ to the excess number of molecules, $\Delta n$, due to the presence of a molecule, through the pair correlation function, $g(r)$. Substitution of Eq. (3.67) into Eq. (3.66) then leads to the nucleation theorem and completes the proof. Thus we have shown that the nucleation theorem holds for a specific nonuniform density distribution that corresponds to an additional molecule at a fixed point and it surroundings, and for this molecule $\partial W / \partial \mu$ is equal the negative of the excess in the compressibility equation of state.

It should be noted that we could have used the nucleation theorem to derive the compressibility equation of state. By simply assuming the validity of the theorem we could have equated Eq. (3.66) with the negative of the left hand side of Eq. (3.67). This would yield the equality on the right hand side of Eq. (3.67) which is the desired result.

It is also worth to mention that the previous analysis can be generalized to include fluctuations that are no longer identical to the molecules in the fluid, but resemble different molecules, as occurs in a multicomponent system [27].

\subsection{The Nucleation Theorem and the Gibbs Adsorp- tion Equation}

The Gibbs adsorption equation can also be derived by using the nucleation theorem where the corresponding constraint is the one creating a large cavity in the fluid. 
We consider that the cavity is a connected region of space that excludes the centers of all the surrounding molecules, so that one having a radius $R_{c}$ fixed at a point $\mathbf{R}$, excludes $(4 / 3) \pi R_{c}^{3} \rho$ molecules. The molecules in the fluid see the "hard wall" of the cavity, and depending on their intermolecular potential will either adsorb or desorb on this surface. In either case, near that surface, there will occur a deviation of the local density away from the original uniform fluid. This deviation will contribute to the molecular excess. Provided that the cavity is large enough that curvature effects can be ignored, the total number of absorbed molecules can be expressed as

$$
\Gamma_{T}=\rho \int_{R_{c}}^{\infty}[g(r)-1] 4 \pi r^{2} d r
$$

where $g(r)$ is the correlation function, at a distance $r$, between the center of the cavity and the molecules in the fluid, while $\rho$ is the original uniform fluid density. The total excess due to the presence of the cavity is a combination of molecules absorbed at the surface and those expelled from the cavity, and can be written

$$
\Delta n=-\frac{4}{3} \pi R_{c}^{3} \rho+\Gamma_{T}=-\frac{4}{3} \pi R_{c}^{3} \rho+4 \pi R_{c}^{2} \Gamma,
$$

where the negative sign in the first term arises because the cavity excludes molecules, so that the local density inside the cavity is lower than the original density. In the second term of Eq. (3.69) $\Gamma_{T}$, the total number of adsorbed molecules has been replaced by $\Gamma$, the thermodynamic surface excess expressed as the number of molecules adsorbed per unit area.

The reversible work required to grow the cavity, again assuming that it is large enough, is given by [26]

$$
W=\frac{4}{3} \pi R_{c}^{3} P+4 \pi R_{c}^{2} \gamma,
$$

where $P$ is the pressure of the fluid and $\gamma$ is the surface or boundary tension of the fluid at the wall interface. The first term is the volume work need to grow the cavity while the second is the work needed to create the new interface. As in the previous section, we take the derivative of this work with respect to the chemical potential and find

$$
\begin{aligned}
\left(\frac{\partial W}{\partial \mu}\right)_{R_{c}} & =\frac{4}{3} \pi R_{c}^{3}\left(\frac{\partial P}{\partial \mu}\right)+4 \pi\left(\frac{\partial \gamma}{\partial \mu}\right) \\
& =\frac{4}{3} \pi R_{c}^{3} \rho+4 \pi\left(\frac{\partial \gamma}{\partial \mu}\right),
\end{aligned}
$$


where in the second step we have made use of the Gibbs Duhem relation. Using the nucleation theorem, taking note of the fact that it requires the negative of the excess, Eqs. (3.69) and (3.70) can then be equated. This gives

$$
\Gamma=-\left(\frac{\partial \gamma}{\partial \mu}\right)
$$

which is the Gibbs adsorption equation for the surface in question. On the other hand, we could have used Eq. (3.71) in Eq. (3.70) to recover the nucleation theorem. This shows that, in the regime where the cavity is large enough to neglect the effects of curvature, the Gibbs adsorption equation is a necessary and sufficient consequence of the nucleation theorem. Also, the form of the surface work that we have chosen indicates that the surface involved is the Gibbs surface of tension. However, for large enough $R_{c}$ its exact location does not have to be specified.

\subsection{The Nucleation Theorem and the Molecular Ex- cess}

At this point, it is appropriate to clarify the relation of the nucleation theorem to the cluster that constitutes the nucleus. There seems to be no doubt that, within the limits of the approximation, if the kinetic preexponential factor in the nucleation rate is independent of the chemical potential, one can determine the "excess" associated with the nucleus. However, it is important to elucidate what is the real meaning of this molecular "excess".

Let us consider for instance a $n$-cluster defined by isolating $n$ particles inside a given volume, following some criteria, i.e. the Stillinger cluster. Then one usually assumes that these $n$ particles constitute the cluster, and the remaining $N-n$ the surrounding vapor. In principle, one could then perform a statistical mechanics description of such droplet and calculate, through the partition function, the work associated to its formation. If the vapor is ideal, in the sense that it does not interact with the $n$ particles inside the volume of the cluster, one would obtain [17]

$$
\left(\frac{\partial W_{c}(n)}{\partial \mu}\right)_{V, T, \text { ideal }}=-(n-\bar{n}) .
$$

That is, in this case the entire excess in number of molecules is contained inside the volume of the cluster and coincide with the $n$ particles we have isolated less the number $\bar{n}$ of ideal vapor molecules that would occupy that volume. Of 
course, if the vapor is not ideal, those $n$ particles inside the volume of the cluster may interact with the surrounding vapor, which originates an additional term to the partition function. This term, following Eq. (3.34), contributes to the work of formation of that droplet and consequently generates a new term $\Delta n$ in the excess

$$
\left(\frac{\partial W_{c}(n)}{\partial \mu}\right)_{V, T, \text { nonideal }}=-(n-\bar{n}+\Delta n)
$$

One then might think that this additional term $\Delta n$ destroy the validity of the nucleation theorem. But this would be a misinterpretation of the real meaning of the molecular excess.

The excess is a property of the whole system and cannot be independent on the election, the location or the properties of the dividing surface. Except in few particular cases, one cannot expect that the entire excess will be located in a restricted region inside the system, as for instance in the volume containing the $n$ particles. The molecular examples developed in the preceding sections clearly illustrates this fact. The density profile outside the cavity in Fig. 3.3 confirms that a significant part of the molecular "excess" is exterior to the cavity. In addition, the calculated excess for the case of the cluster containing 2 hard-rods is not simply $n-\bar{n}=2-\rho \lambda$, but is given instead by Eq. (3.60).

Even the Stillinger cluster will exhibit an "excess" outside of the cluster itself unless its surroundings are almost ideal. This exterior excess, $\Delta n$, forms part of the total excess that the nucleation theorem refers to. Moreover, it corresponds to the term that Bowles et al. [17] could not force to zero in their molecular analysis of the problem. We now know that, in the first place, there was no need to explain how this term could be zero since it represented an excess that was to be included in the theorem.

Once elucidated the real meaning of the molecular excess given by the nucleation theorem, one may take one step forward. One of the difficulties in the development of nucleation theories is the definition of a physically consistent cluster. As we showed in Chapter 1, many definitions have been proposed in the literature, but only a few criteria, as for example the comparison with experimental results, are available to show their accuracy. The nucleation theorem becomes now a valuable tool to refine nucleation theories and cluster models. The last discussion suggests that a "good" definition of a physically meaningful cluster would be the one that never leads to an appreciable exterior excess, even when the cluster is embedded in a non ideal environment. 


\subsection{Conclusions}

In concluding this chapter, it is appropriate to carefully examine what has been accomplished in the previous sections, and to speculate on future directions that might be profitable.

First, we have presented a proof that the nucleation theorem applies to any system having an equilibrium nonuniform distribution of density induced by an external field. The case of the cluster of molecules constituting the nucleus in nucleation theory is only a particular case. (We also noted that the theorem is identical to a set of thermodynamic relations derived by Hill in 1962 in the framework of small system thermodynamics). This proof also shows that the theorem extends rigorously down to nonuniformities of molecular dimensions. Furthermore the theorem, as it emerges from the proof, is relatively sterile until it is used in connection with a particular real thermodynamic system or a particular model of a real system. The ingredients of such a model involve the specification of the independent thermodynamic variables of the system, the nature of the field that induces the nonuniformity, or the character of the nonuniformity itself. This character may involve the assumption that the density profile is mesoscopic and local as in the case of a drop. It may also be determined by some underlying molecular restrictions, as for example in the case of a Stillinger cluster. The nature of the density profile (and the corresponding field) might be determined by the presence of a wall or it might correspond to the field of a single molecule, as in the case of a pair correlation function.

Not surprisingly, the nucleation theorem has already been used extensively within the nucleation community, where it provides an essential link between experiment and theory by allowing the molecular excesses predicted by molecular models and computer simulation to be compared with those obtained directly from nucleation rate data. However, in the present chapter we have been able to show that the so-called nucleation theorem is applicable beyond the field of nucleation since it constitutes a general thermodynamic relation. To highlight this we have applied the theorem (i) to the molecular excesses due to cavities and clusters in hard rod systems, and (ii) to the excesses surrounding monoatomic molecules of any type in fluids, even in the three-dimensional case. We have also shown a close connection, at the molecular level, between the nucleation theorem and the compressibility equation of state, and at the macroscopic level between the nucleation theorem and the Gibbs adsorption equation.

Moreover, it is important to emphasize that relations resembling the nucleation theorem have been widely used in many fields other than nucleation, without considering that its formulation deserved a distinctive label. For instance, 
it has come to the authors' attention that A. Vrij [28] derived the compressibility equation of state from the Gibbs adsorption equation using an intermediate relation that was, in effect, the "nucleation theorem". Another example can be found in Ref. [29], where similar relations where applied for solvation forces in fluids confined between parallel walls.

Given the general nature of the theorem, it would be reasonable to identify it by a name that does not seem to restrict it to nucleation. However, it is perhaps too late for this and it might confuse the issues. In view of the generality of the theorem, it could represent a powerful tool for the exploration of a wide range of phenomena different from those associated with nucleation. For example, the nucleation theorem might be useful in the study of transition state complexes; after all the nucleus is a transition complex in chemical kinetic language. It could also be useful in the study of solutions where the solvation of a solute molecule forms part or all of the "excess" of solvent. This application might be especially useful in the study of water molecules in the atmosphere of a polyelectrolyte molecule. As far as theory is concerned, the nucleation theorem might be helpful, for example, in achieving closure in integrodifferential theories of fluids and, in particular, in scaled particle theory [26]. 


\section{Bibliography}

[1] M.P. Anisimov and A.G. Cherevko, Izv. Akad. Nauk USSR (Siberian Branch) (ser. Khim Nauk) 2, 15 (1982).

[2] D. Kashchiev, J. Chem. Phys. 76, 5098 (1982).

[3] Y. Viisanen, R. Strey, and H. Reiss, J. Chem. Phys. 99, 4680 (1993).

[4] W. Thomson, Proc. R. Soc. Edinburgh 7, 63 (1870).

[5] R. Strey, P.E. Wagner, and Y. Viisanen, J. Phys. Chem. 98, 7748 (1994).

[6] D.W. Oxtoby and D. Kashchiev, J. Chem. Phys. 100, 7665 (1994).

[7] P.R. Ten Wolde and D. Frenkel, J. Chem. Phys. 109, 9901 (1998).

[8] K.J. Oh and X.C. Zeng, J. Chem. Phys. 110, 4471 (1999).

[9] D. Kashchiev, Nucleation and Atmospheric Aerosols 2000, AIP Conf. Proc. 534, 147 (2000).

[10] I.J. Ford, J. Chem. Phys. 105, 8342 (1996).

[11] M. Knott, H. Vehkamäki, and I.J. Ford, J. Chem. Phys. 112, 5393 (2000).

[12] H. Vehkamäki and I.J. Ford, J. Chem. Phys. 114, 5509 (2001).

[13] R. McGraw and A. Laaksonen, Phys. Rev. Lett. 76, 2754 (1996).

[14] P.R. Ten Wolde, Numerical Study of Pathways for Homogeneous Nucleation (Academisch Proefschirft, 1998).

[15] J.W. Gibbs, The Scientific Papers of J. Willard Gibbs(Dover, New York, 1961). 
[16] D. Kashchiev, Nucleation. Basic Theory with Applications (Butterworth Heinemann, Oxford, 2000).

[17] R.K. Bowles, R. McGraw, P. Schaaf, B. Senger, J.-C. Voegel, and H. Reiss, J. Chem. Phys. 113, 4524 (2000).

[18] T.L. Hill, J. Chem. Phys. 36, 3182 (1962).

[19] T.L. Hill, Thermodynamics of Small Systems: Parts I and II (W. A. Benjamin Inc., New York, 1963 and 1964).

[20] T.L. Hill, Statistical Mechanics: Principles and Selected Applications (McGraw-Hill, 1956).

[21] H. Reiss, Methods of Thermodynamics, (Dover, New York, 1965).

[22] L.D. Landau and E.M. Lifshitz, Course of Theoretical Physics Vol 5 (Statistical Physics Part 1).

[23] L. Tonks, Phys. Rev. 50, 955 (1936).

[24] H. Reiss, H.L. Frisch, and J.L. Lebowtiz, J. Chem. Phys. 31, 369 (1959).

[25] E. Helfand, H.L. Frisch, and J.L. Lebowitz, J. Chem. Phys. 34, 1037 (1961).

[26] H. Reiss, H.L. Frisch, E. Helfand, and J.L. Lebowitz, J.Chem. Phys. 32, 119 (1960).

[27] R.K. Bowles, D. Reguera, Y. Djikaev, and H. Reiss, J.Chem. Phys. 115, 1853 (2001).

[28] A. Vrij, Proceedings of the Koninklijke Nederlandse Adademie van Wetenschappen, Series B 88, 221 (1985).

[29] R. Evans and U. Marini Bettolo Marconi, J. Chem. Phys. 86, 7138 (1987). 


\section{Chapter 4}

\section{Nonequilibrium Translational-Rotational Effects}

One of the most controversial issues in nucleation theory is the proper accounting for embryo degrees of freedom, sometimes referred to as the translationalrotational paradox. The controversy dates back to the consideration of Lothe and Pound [1] of what appears to be a serious inconsistency in the conventional theory of nucleation from the vapor phase. These authors pointed out that several important contributions to the free energy of formation of the critical cluster attributable to rotational and translational degrees of freedom had been neglected in Classical Nucleation Theory (CNT). The paradox emerged because the inclusion of these additional terms in the way they proposed, although aimed to restore the consistency of the CNT, increased the nucleation rate by a factor on the order of $10^{17}$, thus destroying any reasonable agreement between theory and experiments.

Reiss and coworkers [2,3] attempted to solve the paradox by arguing that the CNT free energy barrier already takes into account all rotational and most of translational contributions. From this point of view, the correction they proposed lead to a much smaller change in the expected rates, between $10^{3}$ and $10^{6}$.

There followed a series of counter arguments and discussions by several authors [4]-[11]. Although the proper inclusion of these contributions continued being debated [11], the issue was never satisfactorily resolved until the recent work of Reiss, Kegel and Katz [12] which clarifies this and other inconsistencies of the nucleation theory.

As we have seen, considerable emphasis has been put on the introduction of the translational and rotational degrees of freedom in the equilibrium partition 
function of a nucleating droplet. However, these corrections refer only to the influence of these degrees of freedom in the equilibrium sense. Translational and rotational degrees of freedom also arise from the motion and rotation of the clusters through the metastable phase. This is a purely nonequilibrium effect, which may have a relevant influence in the nucleation kinetics.

Our purpose in this chapter is precisely to analyze the influence of the translational and rotational degrees of freedom in nucleation, stressing out the mesoscopic and nonequilibrium nature of the process [13]. A large amount of work has been done concerning the equilibrium aspects of those degrees of freedom and it is not our intention to provide here a comprehensive review of the many attempts to solve the paradox which would lie beyond the scope of this thesis. We will try instead to clarify the origin and the resolution of the paradox through a proper interpretation of the mesoscopic nature of the process. That will be developed in the first part of this chapter.

The main objective of this chapter is the analysis of the nonequilibrium effects corresponding to the motion of the clusters in nucleation. To this end, we will first derive the equation governing the kinetics of the nucleation, retaining the dynamics of the clusters, and using the mesoscopic nonequilibrium thermodynamics scheme developed in Chapter 2. After a proper elimination of the velocity variables, this equation will be used to obtain a purely nonequilibrium correction to the nucleation rate. We will finally discuss explicitly the particular case of nucleation rates of water and the extension and relevance of our results in other situations.

\subsection{Influence of Translational-Rotational Degrees of Freedom in Nucleation}

As we showed in Chapter 1, phenomenological theories of nucleation are based on the formulation of the free energy $\Delta G(n)$ associated to the formation of a cluster of $n$ molecules from the metastable phase. The expression (1.21) proposed by CNT, based on capillarity approximation, is obtained by treating the cluster as a macroscopic spherical droplet with bulk and surface free energy contributions. However, treating embryos as macroscopic objects and using thermodynamic arguments to calculate the work of embryo formation give rise to important inconsistencies $[14,15]$. One of these is that logarithmic corrections, like the ones arising from additional degrees of freedom that a drop may have, are not included. In particular, the CNT leaves out the contribution of the translational and rotational degrees of freedom. 
Capillarity approximation deals with the free energy required to form a single cluster at rest in the metastable phase. However, the conception of droplets at rest constitutes an approximation to the real state of the system. The small clusters which are the embryos of the new phase can spontaneously appear at any point of the system, and with arbitrary orientation. Moreover, due to the mesoscopic size of these entities, they move and spin around the metastable phase because the influence of the medium in which they are embedded (Brownian motion). Both factors constitute translational and rotational degrees of freedom of the cluster and must be taken into account to describe accurately the nucleation process. But the influence and the way of considering both effects is different. The arbitrariness of positions and orientations which a cluster may occupy can be incorporated as a purely equilibrium correction by including these translational and rotational degrees of freedom in the partition function of the cluster. Consequently, this yields a modification of the free energy of formation of this cluster. Contrarily, the effect of the movement and rotation of the clusters does not directly alter the nucleation barrier itself. It is a nonequilibrium factor that modifies the kinetics of the process. Both aspects will be discussed separately.

\subsubsection{Equilibrium Influence: The Translational-Rotational Paradox}

Most of the work on translational-rotational correction, and the paradox itself, addresses to modify the free energy to form a cluster of size $n$, taking into account those degrees of freedom in the partition function. In this sense, one may refer to the works of Lothe and Pound (LP)[1], which originated the paradox, and the recent resolution by Reiss, Kegel and Katz (RKK)[12]. Both works deal with purely equilibrium corrections. The common underlying idea is that if nucleation occurs in a volume $V$, the physical critical cluster may appear anywhere in the system with any orientation. Considering that all the clusters appearing at different positions and orientations are equivalent, one then has to account for the additional translational and rotational degrees of freedom in the partition function. The accounting of these degrees of freedom increases the value of the partition function, consequently reducing the free energy of formation of a droplet and thus increasing the nucleation rate. The difference between both treatments and the origin of the paradox concerns what is the proper accounting of those degrees of freedom in the partition function and how to define and distinguish different states of a cluster in a volume $V$. The paradox can be reconsidered and understood from a mesoscopic perspective. 


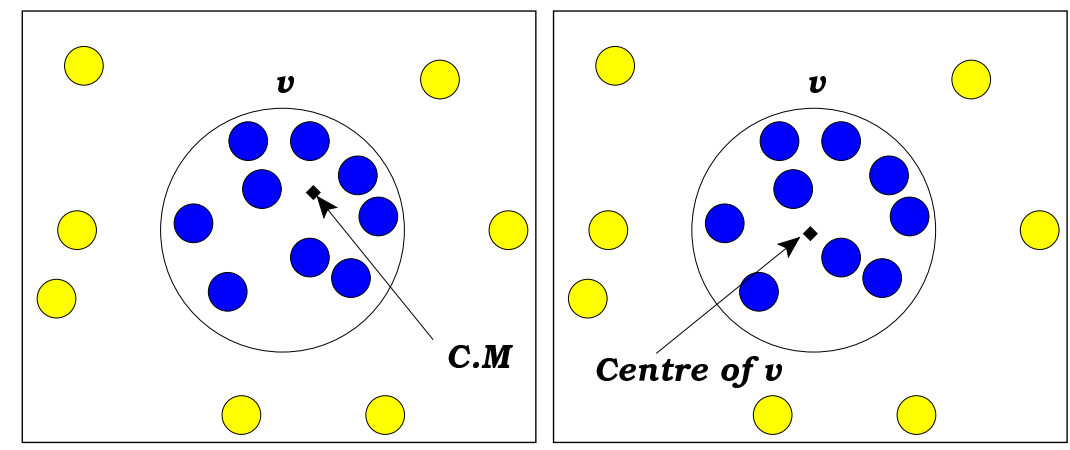

Figure 4.1: Two possibilities of localization of a mesoscopic cluster: left: by the position of the center of mass (C.M); right: by the location of the center of the volume $v$ containing the $n$ molecules.

Using the CNT expression for the free energy of formation of a cluster entails the extrapolation of a macroscopic expression to the mesoscopic scale. But, as became clear in the previous chapter, the extrapolation of macroscopic thermodynamic concepts to the mesoscopic regime is not always straightforward and may give rise to several problems. Some concepts and definitions which are clear in the macroscopic regime, become fuzzy and ambiguous when applied to the mesoscopic level. One of them is precisely the proper description of a mesoscopic entity.

Whereas it is clear how to locate a macroscopic droplet at rest, the characterization becomes less obvious when this cluster only contains a small number of molecules. For instance, the position of a mesoscopic cluster constituted by $n$ molecules inside a spherical volume $v$, can be described in terms of the position of its center of mass, or by the position of the center of the spherical boundaries, as illustrated in Fig. 4.1. Whereas for a macroscopic droplet both descriptions practically coincide, for the mesoscopic one they differ due to the presence of fluctuations.

The other problem arising in the mesoscopic scale concerns the pertinent volume scale one has to use to discretize the space with the purpose of distinguishing different states in the calculation of the partition function. The uncertainty in defining a volume scale is a well-know problem in Statistical Mechanics [16]. For macroscopic systems, however, this arbitrariness is not relevant, because it only introduces a logarithmic correction to the free energy which is always negligible in the thermodynamic limit. But for a small embryo, the correction may become important. 
Hence the central point in the paradox can be reformulated in terms of knowing what is the proper definition of a mesoscopic cluster and what is the physical meaningful volume scale to count states in the partition function and consequently to calculate the free energy of a mesoscopic cluster.

A reasonable choice for the volume scale would be of course based on the quantum uncertainty principle and the corresponding de Broglie wavelength; that was the one implicitly used by Lothe and Pound [1]. They assumed that the location of a spherical cluster of $n$ molecules is defined through the position of its center of mass and its orientation. Therefore every configuration of $n$ molecules with different position of the center of mass and different orientation constitute a different realization of a cluster and consequently a new state to be accounted for in the partition function. Treating the cluster as a spherical macromolecule and applying the quantum uncertainty principle, the quantities $\Lambda=\frac{h}{\left(2 \pi m k_{B} T\right)^{1 / 2}}$ and $\Lambda_{\text {rot }}=\frac{h}{\left(2 \pi I k_{B} T\right)^{1 / 2}}$ represent the smallest length and angular position within which a cluster of mass $m$ and moment of inertia $I$ can be definitely located at a temperature $T$ ( $h$ is the Planck's constant). That is, every configuration of $n$ molecules whose center of mass and orientation differs by $\Lambda$ and $\Lambda_{\text {rot }}$, respectively, is a new state that increases the value of the partition function. This criterion leads to the following correction of the free energy

$$
\Delta G_{L P}(n)=-k_{B} T \ln \frac{V}{\Lambda^{3}}-k_{B} T \ln \frac{8 \pi^{2}}{\Lambda_{r o t}^{3}}-k_{B} T \ln \frac{1}{q_{r e p}},
$$

where the first and the second terms are the translational and rotational correction, respectively; and the last one includes a "replacement factor" $q_{r e p}$ related to the entropy reduction accompanying the separation of $n$ molecules from the system. Assuming reasonable values for parameters concerning vapor condensation, the small size of the de Broglie lengths, originates that these additional terms increase nucleation rates by approximately $10^{17}$, destroying in most cases the agreement between CNT results and experimental data.

But the quantum scale is not the only reasonable scale one may choose, and RKK theory [12] proposes a different alternative. From their point of view, the location of a mesoscopic cluster must be defined by the position of the spherical container, and not by its center of mass. All possible configurations of $n$ molecules inside a spherical container of volume $v$ are then realizations of the same cluster and consequently they are already accounted for in the CNT expression of the free energy. In particular, all possible orientations of the molecules inside the spherical volume are included, which implies that the rotational degrees of freedom are already accounted for in the CNT free energy barrier. 
In addition, to incorporate the translational degrees of freedom one has to take into account that not all the different positions which this spherical cluster may occupy inside the volume of the system correspond to different clusters. As Fig. 4.2 illustrates, if we displace the spherical container a small distance $d x$, most of the configurations of the $n$ molecules compatible with the new situation are the same and have been already accounted for in the undisplaced original cluster. Hence treating this new position of the cluster as a completely different cluster entails an overcounting of states in the partition function.

Those authors affirm that this is essentially the origin of the excessively high correction of obtained by LP which initiated the paradox: the overcounting of configurations in choosing the quantum length scale to enumerate states in the partition function. Consequently, the quantum scale seems not to be the pertinent criteria for a mesoscopic system. There must exist a distinct minimum volume scale to differentiate clusters in the configurational space. The detailed and elegant calculation of the partition function performed by RKK ends up with an interesting conclusion, a kind of analog of the uncertainty principle in the mesoscopic scale. The result is that for a very large drop the proper volume scale is of the order of the mean volume per molecule $\frac{k_{B} T}{P}$. That is meaningful, because even for a big cluster we need to displace our cluster at least to include a different molecule to obtain a different cluster. In addition, for a mesoscopic nearly incompressible drop, this scale turns out to be the one related to the volume fluctuation $\sqrt{k_{B} T \kappa v_{1} n}$. From the mesoscopic point of view that reflects the reasonable fact that a cluster cannot be located more precisely than its volume fluctuation. This criterion prevents the overcounting of configurations who originated the paradox and gives rise to a correction in the nucleation barrier

$$
\Delta G_{R K K}=-k_{B} T \ln \frac{V}{\sqrt{k_{B} T \kappa v_{1} n}}
$$

leading to a much smaller change in nucleation rates on the order of $10^{4}$.

Notice that this criterion is also more consistent with the results of the former chapter. One of the main conclusions of our previous chapter was that the identification of thermodynamic quantities with mesoscopic entities can only be taken into account in an averaged sense. That is, the thermodynamic work of formation of a cluster refers to all possible configurations compatible with a given constraint and not to one particular realization, as the LP treatment implicitly assumed. In particular, in the mesoscopic range, it must include the fluctuations. That is essentially what the RKK criteria is telling us, and the hint to the proper understanding of the paradox. 


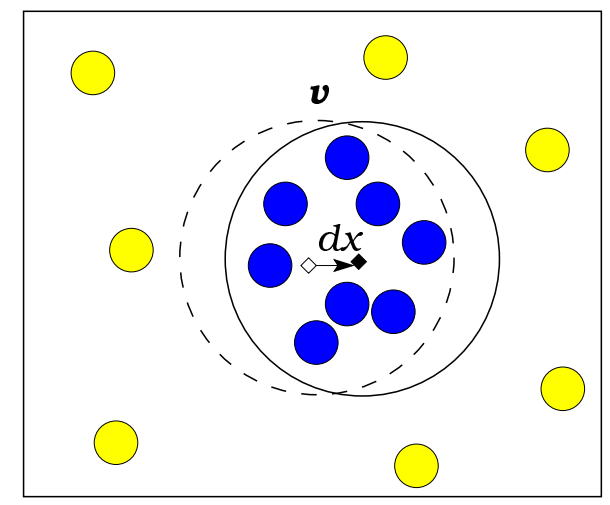

Figure 4.2: Example of two different positions of a cluster sharing the same configuration of molecules. Dashed circle represents the cluster in the original position, and the full circle represents the drop displaced by a distance $d x$.

\subsubsection{Nonequilibrium Aspects: The Effects of Motion}

Both LP and RKK approaches are deeply rooted in equilibrium statistical arguments. However, the correction they propose and the whole controversy around the paradox does not in fact concern the movement of clusters. Although in the literature the effect of the movement of the clusters has been practically ignored, different ways exist for which this movement can influence the process.

On one hand, the movement of the clusters through the metastable phase may alter the rate at which the cluster collides and incorporates molecules of the metastable phase. As we saw in the introduction, the coefficient $k^{+}(n)$ is usually calculated using kinetic theory and is just the number of collisions with molecules of the supersaturated vapor that the cluster suffers per unit time. The classical theory assumes a sticking coefficient equal to 1 (that is, all molecules that collide with the cluster get attached to it) assuming that only the monomers move while the cluster keeps at rest. Olson and Hamill [17] repeated the kinetic calculation considering the effect of the movement of clusters in the supersaturated vapor in the rate at which a cluster gains monomers. The result is a correction factor $\left(1+n^{-1}\right)^{1 / 2}$ in $k^{+}(n)$. However, this correction has a negligible effect on the nucleation rate [15].

But on the other hand, the movement of the cluster can modify not only the rate of growth of a cluster, but also the kinetics of the whole process. Contrarily to the equilibrium point of view, our objective will be to analyze the influence of the movement and rotation of clusters in the nucleation process from a dynamical point of view. Nucleation is a nonequilibrium kinetic phe- 
nomenon essentially related to the variation of the size distribution of clusters present in the system. If the clusters are moving through the metastable phase, its movement may influence the evolution of the cluster size distribution thus altering the kinetics of the process. Therefore, it seems reasonable to study the process not only by considering the evolution of the size of clusters but also retaining its dynamics. This is the approach we develop in the next section, following the mesoscopic nonequilibrium thermodynamic scheme introduced in Chapter 2.

\subsection{Nonequilibrium Kinetics of Nucleation}

We will model the real system, composed by droplets and molecules of the metastable phase, as a dispersion of clusters of different and varying sizes. Since we will focus on homogeneous nucleation in spatial homogeneous systems, the spatial variables are not relevant and will not be included in our description. Thus, the variables characterizing a cluster are its size $n$, its velocity $\mathbf{u}$ and its angular velocity $\mathbf{w}$. The description of the system will be carried out in terms of the distribution function $f(\underline{\Gamma}, t)$, with $\underline{\Gamma} \equiv(n, \mathbf{u}, \mathbf{w})$, whose evolution is dictated by the continuity equation

$$
\frac{\partial f(\underline{\Gamma}, t)}{\partial t}=-\frac{\partial}{\partial \underline{\Gamma}} \cdot \underline{J}(\underline{\Gamma}, t)
$$

where $\underline{J} \equiv\left(J_{n}, \mathbf{J}_{u}, \mathbf{J}_{\omega}\right)$ is a multidimensional current defined in $\underline{\Gamma}$-space.

The nucleation process may be conceived as a diffusion process in the space spanned by the values of $\underline{\Gamma}$ through the energy barrier

$$
C(\underline{\Gamma})=\Delta G(n)+\frac{1}{2} m(n) u^{2}+\frac{1}{2} I(n) \omega^{2},
$$

which is simply the energy of cluster of size $n$, mass $m(n)=n m_{1}$ and moment of inertia $I(n)$, moving with velocities $\mathbf{u}$ and $\mathbf{w}$. In the above expression, $\Delta G(n)$ represents the energy of formation of a $n$-cluster at rest and the remaining terms correspond to translational and rotational kinetic energies. Assuming that the cluster is nearly a rigid spherical object, its moment of inertia would be $I(n)=\frac{2}{5} m(n) r^{2}$. But for our analysis, the really relevant property is the dependency on the size $n$, which in this case is $I(n) \sim n^{5 / 3}$.

The entropy of the system, $S(t)$, is given through the Gibbs entropy postulate $[18,19]$ 


$$
S(t)=-k_{B} \int f(\underline{\Gamma}, t) \ln \frac{f(\underline{\Gamma}, t)}{f_{e q}(\underline{\Gamma})} d \underline{\Gamma}+S_{e q}
$$

where $S_{e q}$ is the value of the entropy at equilibrium. Its variations can be expressed in the form

$$
\delta S(t)=-\frac{1}{T} \int \mu(\underline{\Gamma}, t) \delta f(\underline{\Gamma}, t) d \underline{\Gamma},
$$

where $\mu(\underline{\Gamma}, t)$ is a generalized chemical potential defined in $\underline{\Gamma}$-space.

$$
\mu(\underline{\Gamma}, t)=k_{B} T \ln \frac{f(\underline{\Gamma}, t)}{f_{e q}(\underline{\Gamma})}+\mu_{e q}
$$

This expression corresponds to the chemical potential of an ideal, i.e. noninteracting, dispersion of clusters in the liquid phase. In the latter equation, $f_{e q}(\underline{\Gamma})$ represents the equilibrium distribution given by

$$
f_{e q}(\underline{\Gamma}) \propto \exp \left(-\frac{C(\underline{\Gamma})}{k_{B} T}\right)
$$

and $\mu_{e q}$ is the chemical potential at equilibrium.

The expression for the current $\underline{J}$ defined in Eq. (4.2) can be obtained from nonequilibrium thermodynamics. The entropy production

$$
\sigma=-k_{B} \int \underline{J} \cdot \frac{\partial}{\partial \underline{\Gamma}} \ln \frac{f}{f_{e q}} d \underline{\Gamma}
$$

follows from Eqs. (4.2), (4.5) and (4.6). The resulting linear law, obtained from the assumption of isotropy and locality in $\underline{\Gamma}$-space [20], is given by

$$
\underline{J}(\underline{\Gamma}, t)=-k_{B} \underline{L} \cdot \frac{\partial}{\partial \underline{\Gamma}} \ln \frac{f}{f_{e q}}
$$

where $\underline{L}$ is the corresponding matrix of Onsager coefficients. This expression can be used in the continuity equation (4.2) thus leading to the Fokker-Planck equation

$$
\frac{\partial f}{\partial t}=\frac{\partial}{\partial \underline{\Gamma}} \cdot\left(\underline{D} \cdot \frac{\partial f}{\partial \underline{\Gamma}}+\frac{\underline{D}}{k_{B} T} \cdot \frac{\partial C}{\partial \underline{\Gamma}} f\right),
$$

where $\underline{D}=k_{B} \underline{L} / f$ is the matrix of diffusion coefficients, which in first approximation can be considered as diagonal, neglecting any coupling between the velocities.

The former equation provides a complete dynamical description of the system in terms of the variables $n, \mathbf{u}$, and $\mathbf{w}$. However, from the analysis of the 
time scales of the system it is easy to realize that in the nucleation time scale the system reaches equilibration in velocities space. The characteristic nucleation relaxation time [21] is given by $\tau_{n} \sim \frac{1}{k^{+}(n)}$ where for the rate $k^{+}(n)$ of addition of one molecule to a droplet of size $n$ we may adopt the classical expression Eq. (1.5).

Similarly, the Brownian translational and rotational time scales are $\tau_{\text {trans }} \approx$ $\frac{m}{6 \pi \eta a}$ and $\tau_{\text {rot }} \approx \frac{I}{8 \pi \eta a^{3}}$, respectively, where $a$ is the radius of the cluster and $\eta$ is the viscosity. For nucleation in liquid-vapor systems, the order of magnitude of these characteristic times is approximately $\tau_{n} \approx 10^{-8} n^{2 / 3}$ for the growth of the cluster, and $\tau_{\text {trans }}, \tau_{\text {rot }} \approx 10^{-13} n^{2 / 3}$ for the velocities. Therefore a clear separation between time scales exists, the condition $\tau_{n} \gg \tau_{\text {trans }}, \tau_{\text {rot }}$ holds, and one can perform an adiabatic elimination of the fast variables (the velocities) [22].

The adiabatic elimination is a well-known procedure to simplify multidimensional Fokker-Planck equations when the decay constants for some variables, called "slow", are much larger than those for other variables, named "fast". Under that conditions, it is then reasonable to assume that the fast variables, in our case the velocities, will decay very rapidly to their equilibrium distribution. Hence, for a time scale large compared to the decay time of the fast variables, the process is then mainly described by the evolution of the slow variable, the size $n$ in our problem. One can then eliminate these fast variables by integrating the Fokker-Planck equation over the equilibrium distribution of the fast variables. For the case of the velocities, this is the familiar Maxwellian distribution.

After performing the adiabatic elimination of velocities, the resulting dynamics is governed by the Fokker-Planck equation

$$
\frac{\partial f(n, t)}{\partial t}=\frac{\partial}{\partial n}\left(\bar{D}(n) \frac{\partial f}{\partial n}+\frac{\bar{D}(n)}{k_{B} T}\left(\frac{d \Delta G(n)}{d n}+4 k_{B} T \frac{1}{n}\right) f\right)
$$

where $f(n, t)=\int f(n, \mathbf{u}, \mathbf{w}) d \mathbf{u} d \mathbf{w}$, and $\bar{D}(n)=\int \underline{D} \rho_{e q}(\mathbf{u}, \mathbf{w}) d \mathbf{u} d \mathbf{w}$ is the diffusion coefficient averaged over the equilibrium distribution $\rho_{e q}(\mathbf{u}, \mathbf{w})$ of the velocities. By comparing now this equation with the usual kinetic equation for nucleation Eq. (1.29), one can then identify the diffusion coefficient $\bar{D}(n)$ with the forward rate $k^{+}(n)$, and obtain the expression for the effective nucleation barrier $\Delta G_{n e w}(n)$

$$
\Delta G_{n e w}(n)=\Delta G(n)+4 k_{B} T \ln n .
$$

The conclusion is that translational and rotational motion of clusters in the 
spatial homogeneous medium introduce an effective additional size-dependent contribution $4 k_{B} T \ln n$ in the nucleation barrier, inherent to the diffusion process and thus independent of the energy of formation of cluster at rest $\Delta G(n)$. It is important to highlight that since this contribution is always positive translationalrotational motion always increases nucleation barrier and consequently reduces nucleation rates, contrarily to the results of the equilibrium corrections which always decrease the nucleation barrier thus increasing the rate. One may wonder about the reasons for this feature. In essence, the difference lies on the treatment of velocity variables.

From the equilibrium point of view, it was always assumed that the velocity and the motion of the clusters does not influence the nucleation process. And the justification was precisely the same than the one we have used to eliminate the velocity variables in our Fokker-Planck equation. The time scale of the velocity relaxation is much faster than the nucleation time scale, so it was assumed that nucleation occurs when velocity distribution is equilibrated thus not influencing the process. Following this line of reasoning, the free energy of formation of clusters is constructed by averaging the contribution of all cluster velocities. The nucleation kinetics is then studied focusing only on the evolution of the cluster size distribution.

In contrast with that line of reasoning, in our treatment we consider that, due to the coupling between the dynamics of $n$ and $(\mathbf{u}, \mathbf{w})$, the elimination of velocities must be performed at the last stage because it influences the kinetics of the whole process. Hence we maintain velocities of the cluster as relevant variables in its description. Instead of constructing an averaged free energy of a drop taking into account all possible velocities, we directly use the free energy to form a $n$-cluster with velocities $\mathbf{u}, \mathbf{w}$ (Eq. 4.3) to study the evolution of the system as well with respect to the size of clusters as their velocities. Finally, we perform an adiabatic elimination of $\mathbf{u}$ and $\mathbf{w}$ from characteristic time scales considerations. The result is a net positive contribution to the nucleation barrier.

If in both cases, the velocity is finally eliminated using the same equilibrium distribution and the same arguments, one then may wonder about the physical origin of the additional effective contribution we obtain. The underlying reason can be naively understood as follows. Although the velocity is initially equilibrated and equilibrates very fast, every time that a cluster gains or looses a molecule not only the size distribution of the clusters is altered, but also the velocity distribution. Therefore, the alteration of cluster size distribution entails a reorganization of velocities distribution through diffusing currents. These currents involve an additional dissipation which has an energetic cost. Conse- 
quently, the growing of the cluster requires more effective energy, because part of that is expended in the reorganization (in the new equilibration) of the velocity distribution. As we will show in the following section, the nucleation rate is thus slowed down.

\subsection{Nucleation Rate}

The consideration of translational and rotational contribution due to the motion of the clusters in the nucleation process then leads to the following expression for the stationary nucleation rate per unit volume, resulting from Eq. (4.11)

$$
J=K \exp \left(-\frac{\Delta G_{n e w}\left(n^{*}\right)}{k_{B} T}\right)
$$

where $n^{*}$ is the size of the critical nucleus obtained from the condition of maximum of the nucleation barrier

$$
\left.\frac{d \Delta G(n)}{d n}\right|_{n^{*}}+4 k_{B} T \frac{1}{n^{*}}=0
$$

and $K$ is the pre-exponential factor which for liquid-gas nucleation, following Eq. (1.26), is given by

$$
K=k^{+}\left(n^{*}\right) \frac{p}{k_{B} T} \sqrt{-\frac{1}{2 \pi k_{B} T} \frac{\partial^{2} \Delta G\left(n^{*}\right)}{\partial n^{2}}} .
$$

In order to quantitatively illustrate the effect of our correction in the nucleation rate, we will particularize our general formulation to a concrete physical situation by using a specific model for the energy barrier $\Delta G(n)$ of formation of clusters at rest. Our aim in this sense is to show how the consideration of the translational-rotational degrees of freedom in the way we propose may lead some phenomenological theories to a better agreement with experimental data for some substances.

As a concrete example, we will focus on the nucleation rates of water. We choose this substance because its thermophysical properties are well-established (see Table 4.1) and there are accurate experimental measures of nucleation rates performed by Viisanen et al. [23]. As a model for the free energy of formation of a cluster at rest $\Delta G(n)$, we will use RKK model, explicitly

$$
\Delta G_{R K K}(n)=-k_{B} T n \ln S+\sigma s_{1} n^{2 / 3}-k_{B} T \ln \frac{V}{\sqrt{k_{B} T \kappa v_{1} n}} .
$$


Table 4.1: Thermophysical properties of the water required to evaluate the nucleation rate: molecular mass $M$; saturation pressure $p_{e q}$ expressed in $m m H g$; liquid density $\rho_{l}$; surface tension $\sigma$; and isothermal compressibility $\kappa$. In the table, $T$ represents the absolute temperature in Kelvins, $\vartheta=T-273.15 K$ is the Celsius temperature and $\epsilon=(T / 228-1)$. Data taken from Refs. [25] and [24].

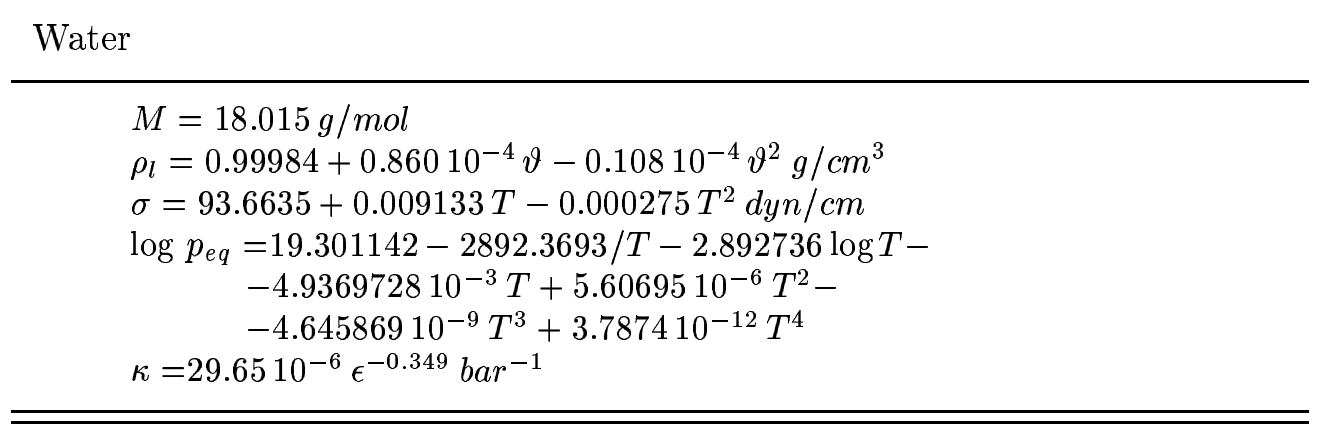

As we have discussed in Section 4.1.1, the RKK model constitutes an improvement of the CNT in the sense that solves some consistency problems and properly incorporates the equilibrium corrections due to the translational degrees of freedom. Unfortunately, this theoretical consistency does not guarantee an improvement of the quantitative results. In fact, for the particular case of water, the RKK theory clearly overestimates the nucleation rate by factor of $10^{5}-10^{6}$, whereas the discrepancy of the CNT is approximately two orders of magnitude (see Figs. 4.3 and 4.4).

However, taking into account the nonequilibrium effects of the motion of the cluster in the way we propose and using the RKK energy barrier one can achieve a very good concordance with the experimental results. In Fig. 4.4, we compare the experimental values of Ref. [23] for the nucleation rate of water at different temperatures, with the predictions of CNT, and the values obtained using Eq. (4.13) from our theory. We can see that the agreement of our result is excellent, specially at high temperatures. For low temperatures, the results get slightly worst which can be attributed to the low temperature anomalies of the compressibility of the supercooled water (in fact it diverges at approximately $228 \mathrm{~K}$, see Ref [24]). Nevertheless, the maximum discrepancies are less than a factor of 3 for the whole range of temperatures above $228 \mathrm{~K}$. This example illustrates the fact that an energy barrier constructed under the requirement of preserving self-consistency and including properly the nonequilibrium effects may reproduce experimental results. 


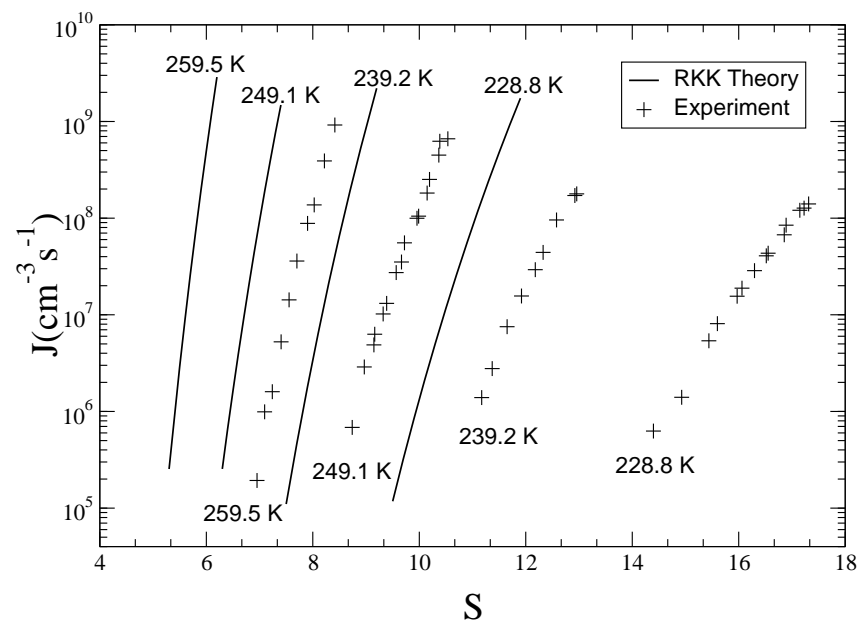

Figure 4.3: Comparison of experimental nucleation rates $J$ as a function of the supersaturation $S$ with the predictions of the RKK Theory (full lines). Crosses represent data of Viisanen et. al. [23] for water at different nucleation temperatures.

It is important to emphasize that the accuracy of the results we may provide mostly relies on the correctness of the energy barrier at rest $\Delta G(n)$ we adopt. The theory we present aims to analyze the nonequilibrium effects of the motion of the clusters in nucleation, but phenomenological nucleation barriers based on capillarity approximation present other serious drawbacks, like dependencies of the surface tension on the radius of cluster or temperature, on which agreement with experiments depends on. It is evident, that in those cases translational corrections are not sufficiently important to reestablish the agreement with experiments.

On what concerns nucleation in liquids, there is a general belief that cluster motion is of little influence and does not need to be considered. Homogeneous nucleation of crystals is much less well understood than condensation discussed previously, and presents additional difficulties [26] as mentioned in Chapter 1. However, in spite of the differences between gas and crystal nucleation, and the additional problems that the latter presents, the formalism previously developed to introduce translation-rotation effects in liquid-gas nucleation remains applicable. Consequently, the net effect of translational-rotational degrees of freedom predicted by our model is again the variation of the energy barrier given in Eq. (4.12). In this case, the differences between our nonequilibrium treatment and the equilibrium approaches become even more obvious, because in condensed phases translational/rotational contributions to the free energy of 


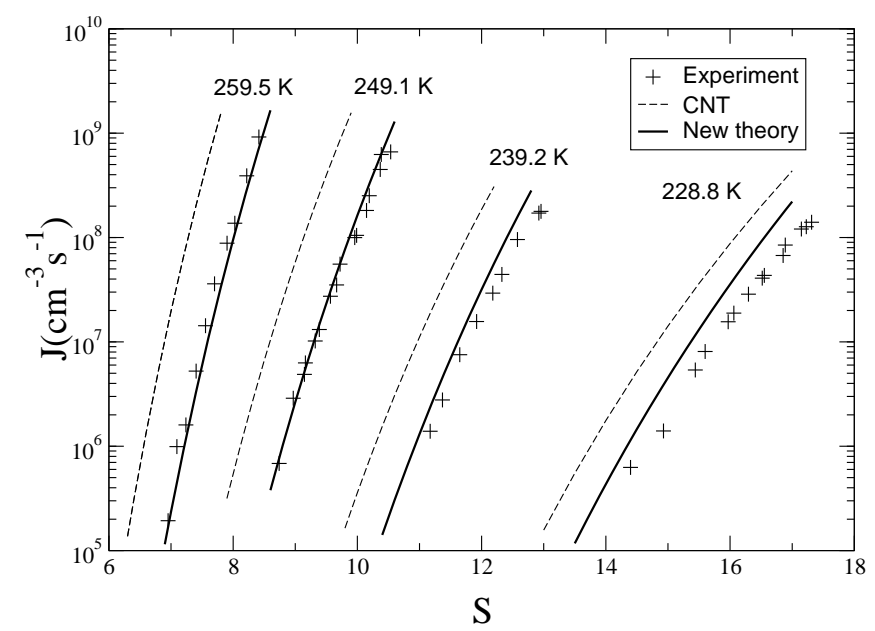

Figure 4.4: Comparison of experimental nucleation rates $J$ as a function of the supersaturation $S$ with the predictions of the Classical Nucleation Theory (dashed lines) and the new theory (full lines). Crosses represent data of Viisanen et. al. [23] for water at different nucleation temperatures.

a cluster (in the partition function) are expected to be negligible [26], while the influence of the movement of clusters in the kinetics of the process still remains the same.

\subsection{Conclusions}

In this chapter we have shown how the proper understanding of the mesoscopic and nonequilibrium nature of the nucleating kinetics reveals the solution of one of the most paradoxical issues in the nucleation theory, the inclusion of the translational and rotational degrees of freedom.

The importance of a pure nonequilibrium description of nucleation becomes then manifest. Our mesoscopic nonequilibrium scheme reveals the transcendence of the effect of the motion of the cluster which has been long-ignored in the studies of nucleation.

The analysis of the problem has been performed by taking into account the fact that the emerging clusters remain embedded in the metastable phase and that their movement could induce modifications in the nucleation rate. Actually, the motion of the clusters influence the kinetics of their size evolution, therefore the study of the problem must be performed by retaining their dynamics. The influence of the medium is then reflected in the appearance of a new effective 
contribution to the energy barrier which is independent of the definition of cluster and of the energy barrier at rest one adopts. The contribution we have found originates from Brownian diffusion of clusters and in this sense is a translational contribution arising from a nonequilibrium situation and not from equilibrium statistical considerations. The effect we have introduced is fundamental as is due to the ineluctable presence of the medium during the nucleation process.

The conclusion is that the motion of the clusters is relevant in the kinetics of nucleation, leads to a reduction of the nucleation rates, and its proper inclusion constitutes a first step toward the construction of a nucleation energy barrier that preserve all logical consistencies and is able to reproduce and predict experimental results.

A relevant aspect to be emphasized is that our analysis could directly be extended to consider other hydrodynamic effects in nucleation processes, as the ones related to the presence of gradients or inhomogeneities. We then provide a theoretical framework from which the influence that the proper dynamics of the metastable phase may play in the nucleation process could be studied systematically. That is the objective of the next chapter. 


\section{Bibliography}

[1] J. Lothe and G.M. Pound, J. Chem. Phys. 36, 2080 (1962).

[2] H. Reiss and J.L. Katz, J. Chem. Phys. 46, 2496 (1967).

[3] H. Reiss, J.L. Katz, and E.R. Cohen, J. Chem. Phys. 48, 5553 (1968).

[4] J. Lothe and G.M. Pound, J. Chem. Phys. 48, 1849 (1968).

[5] R. Kikuchi, J. Stat. Phys. 1, 351 (1969).

[6] J. Lothe and G.M. Pound, in Nucleation (A. C. Zettlemoyer, ed.) p. 109, Marcel Dekker, New York (1969).

[7] M. Blander and J.L. Katz, J. Stat. Phys. 4, 55 (1972).

[8] K. Nishioka and G.M. Pound, Adv. Colloid Interface Sci. 7, 205 (1977).

[9] H. Reiss, Adv. Colloid Interface Sci. 7, 1 (1977).

[10] R. Kikuchi, Adv. Colloid Interface Sci. 7, 67 (1977).

[11] V. Ruth, J.P. Hirth, and G.M. Pound, J. Chem. Phys. 88, 7079 (1988).

[12] H. Reiss, W.K. Kegel, and J.L. Katz, Phys. Rev. Lett. 78, 4506 (1997); J. Phys. Chem. A 102, 8548 (1998).

[13] D. Reguera and J.M. Rubí, J. Chem. Phys. 115, 7100 (2001).

[14] A. Laaksonen, V. Talanquer, and D.W. Oxtoby, Annu. Rev. Phys. Chem. 46, 489 (1995).

[15] D.T. Wu, Solid State Phy. 50, 37 (1996).

[16] T.L. Hill, Statistical Mechanics (Dover, New York, 1987), pag. 62-64. 
[17] T. Olson and P. Hamill, J. Chem. Phys. 104, 210 (1996).

[18] J.M. Rubí and P. Mazur, Physica A 250, 253 (1998).

[19] D. Reguera, J.M. Rubí, and A. Pérez-Madrid, J.Chem. Phys. 109, 5987 (1998).

[20] S.R. de Groot and P. Mazur, Non-Equilibrium Thermodynamics, (Dover, New York, 1984).

[21] G. Shi, J.H. Seinfeld, and K. Okuyama, J. Appl. Phys. 68, 4550 (1990).

[22] H. Risken, The Fokker-Planck Equation, (Springer, Berlin, 1984).

[23] Y. Viisanen, R. Strey, and H. Reiss, J. Chem. Phys. 99, 4680 (1993); ibid 112, 8205 (2000).

[24] R.J. Speedy and C.A. Angell, J. Chem. Phys. 65, 851 (1976).

[25] A. Dillmann and G.E. Meier, J. Chem. Phys. 94, 3872 (1991).

[26] K.F. Kelton, Solid State Phy. 45, 75 (1991). 


\section{Chapter 5}

\section{Homogeneous Nucleation in Inhomogeneous Media}

The analysis developed in the previous chapters has been focused on the simplest case of homogeneous, isothermal and isotropic nucleation. But the real situation under which nucleation occurs very often does not correspond to that conditions. One has to take into account that the real process occurs in a media, which in general has spatial, thermal or velocity inhomogeneities, which in turn may exert a relevant influence in the process.

One of the situations in which this fact becomes more evident is in polymer crystallization, which will be discussed in the next chapter. Contrarily to the case of solidification of simple substances, crystallization of polymers occurs in a wide range of temperatures. Moreover, in realistic situations the external conditions, specially those concerning the temperature of the system, may change in time thus altering the process significantly. These characteristics makes it necessary the study of the process under non-isothermal and inhomogeneous conditions.

Our aim in this chapter is precisely to develop a more realistic model of nucleation and crystallization which considers the potential influence of the medium. We will focus on the simplest cases in which the medium may affect the kinetics. These situations involve the presence of spatial inhomogeneities in the thermodynamic properties or the conditions under which nucleation occurs; the presence of temperature gradients or non-isothermal conditions; and finally the impact of flow and stresses in the nucleation process.

In the case of homogeneous isotropic nucleation, we can leave spatial dependencies aside as the process occurs identically at any point of the system. We

can thus focus our description on the evolution of the cluster size as a function 
of the external conditions (pressure, temperature, density). The homogeneity thus justifies the use of a global thermodynamic approach.

However, when the system is inhomogeneous the conditions controlling condensation and crystallization vary from point to point of the material and therefore a local description of the process must be considered.

To this purpose, we will divide the whole sample into volume elements small enough in order that temperature, pressure, density... can be considered as homogeneous, but large enough to guarantee that thermodynamics can still be applied. In terms of length scales, the previous statement means that the typical length of the volume element must be much smaller than the gradients present at the system and much bigger than any microscopic length.

In order to guarantee the consistency of a thermodynamic analysis at the local level, we must introduce the local equilibrium hypothesis. This hypothesis assumes that, although the system is not globally in equilibrium, thermodynamics holds locally. That is, the local variables density, temperature, etc.. are related through the same thermodynamic relationships than those for homogeneous macroscopic systems. In particular, the Gibbs equation remains valid locally for a volume element followed along its center of gravity motion [1].

It is important to remark, that nucleation involves two clearly differentiated length scales of macroscopic and mesoscopic natures. On one hand, the scale in which thermodynamic quantities as pressure, temperature, density, etc. vary. These quantities can be considered as uniform within each elementary cell, but may change along the sample. On the other hand, nucleation occurs on a mesoscopic scale.

Once again, the mesoscopic nonequilibrium thermodynamics (MNET) [2][11] becomes the natural framework to analyze this process. The key ingredient to apply this formalism to the present situation is to carry out a local description resorting to a reference state in which local equilibrium is assumed to hold.

Starting from this reference state we will use our MNET formalism developed in the second chapter in order to obtain the Fokker-Planck equation describing the dynamics of this kind of "equilibrium fluctuations". Following the tenets of nonequilibrium thermodynamics, we will assume that any nonequilibrium initial state will decay to the reference state in the same way as an equilibrium fluctuation. That is, the evolution of nonequilibrium states is also dictated by the same Fokker-Planck equation.

The resulting Fokker-Planck equation provides a complete description of the system at the mesoscopic level. However, it contains more information than the one accessible from experiments, which are performed on a macroscopic scale. We will then describe the macroscopic evolution of the system through 
the hydrodynamic equations, which are constructed by averaging the relevant quantities with the probability density occurring in the Fokker-Planck equation.

In this way, we will obtain the kinetic equations governing the nucleation and the transport in the system. We will point out that the formalism is able to recover some results of the kinetic theory, in a more simple and systematic way, inside a nonequilibrium thermodynamics framework. In particular, we can obtain relaxation equations and describe the short-time inertial regime of the dynamics. However, for the nucleation problem, this short-time regime is usually not relevant, hence we will focus our analysis on a long-time regime where diffusion is the dominant mechanism of transport.

For simplicity, we will not consider angular variables in the analysis developed in this chapter. These can be easily included following our general scheme, but their inclusion complicates considerably the description of the process. For the sake of concreteness, we will analyze in detail two particular interesting situations. First we will derive the kinetic equations governing nucleation in spatially inhomogeneous systems under the presence of a temperature gradient. The second case under scrutiny will be nucleation in a stationary flow, representative of the influence of stresses or mechanical processing. These examples resemble situations of real interest. Using the results obtained within our theoretical framework, we will finally discuss the implications in condensation experiments and in polymer crystallization [12].

\subsection{Nucleation under Spatially Inhomogeneous Non-Isothermal Conditions}

Let us consider a non-isothermal situation where the medium, globally at rest, is characterized by a temperature profile $T(\mathbf{x})$. In our reference state, this profile is assumed as stationary.

To account for spatial inhomogeneities, we will perform a local description in terms of $f(n, \mathbf{x}, \mathbf{u}, t) / N$, the probability density of finding a cluster of size $n \in(n, n+d n)$ at $\mathbf{x} \in(\mathbf{x}, \mathbf{x}+d \mathbf{x})$, with velocity $\mathbf{u} \in(\mathbf{u}, \mathbf{u}+d \mathbf{u})$, at time $t$. $N$ is the total number of clusters in the system. The whole system is then thermodynamically characterized by the local energy density per cluster $e(\mathbf{x}, t)$, the entropy density per cluster $s(\mathbf{x}, t)$, and the number density

$$
\rho(\mathbf{x}, t)=\rho_{m}+\rho_{c} \equiv \rho_{m}+\int f(n, \mathbf{x}, \mathbf{u}, t) d \mathbf{u} d n
$$

where $\rho_{m}$ is the density of the heat bath, assumed constant. 
Our initial step is the formulation of the Gibbs equation for the entropy variations of this system, which is now expressed in terms of the corresponding local thermodynamic quantities (see Appendix)

$$
\delta(\rho s)=\frac{\delta(\rho e)}{T}-\frac{1}{T} \int \mu(n, \mathbf{x}, \mathbf{u}, t) \delta f(n, \mathbf{x}, \mathbf{u}, t) d \mathbf{u} d n,
$$

where $\mu(n, \mathbf{x}, \mathbf{u}, t)$ is the generalized chemical potential. Notice that the last term in the previous equation is reminiscent of the corresponding one for a mixture in which the different components would be specified by the continuum 'indexes' $n$ and $\mathbf{u}$. The generalized chemical potential can be specified through the use of the Gibbs' entropy postulate. This formula establishes the connection between statistical mechanics and thermodynamics through the definition of the entropy in terms of a probability function

$$
\rho s=-k_{B} \int f \ln \frac{f}{f_{l e q}} d \mathbf{u} d n+\rho s_{l e q},
$$

being $f_{l e q}$ and $\rho s_{l e q}$ the probability distribution and the entropy at local equilibrium, respectively. Variations of this expression yield:

$$
\delta(\rho s)=-k_{B} \int \delta f \ln \frac{f}{f_{l e q}} d \mathbf{u} d n+\delta(\rho s)_{l e q},
$$

where the variations of the local equilibrium entropy obey the Gibbs equation

$$
\delta(\rho s)_{l e q}=\frac{1}{T} \delta(\rho e)_{l e q}-\mu_{l e q} \delta \rho_{l e q}
$$

and $\mu_{l e q}(\mathbf{x})$ denotes the local equilibrium chemical potential which is independent of the internal variables, but in general will depend on position. By comparison of the previous equation with the Gibbs equation (5.2), one obtains the expression for the generalized chemical potential

$$
\mu(n, \mathbf{u}, \mathbf{x}, t)=k_{B} T \ln \frac{f}{f_{l e q}}+\mu_{l e q}(\mathbf{x}) .
$$

At local equilibrium, the system (i.e. the ensemble of clusters) is described at each position by an equilibrium distribution function of the internal variables (i.e the velocities and sizes) in which the temperature is the corresponding one at that point. Consequently, the local equilibrium distribution is just the probability of formation of an equilibrium fluctuation originating a cluster of $n$ particles and velocity $\mathbf{u}[13]$ 


$$
f_{l e q}(n, \mathbf{u}, \mathbf{x}, t)=\exp \left(\frac{\mu_{l e q}-C(n, \mathbf{u})}{k_{B} T}\right) .
$$

The quantity $C(n, \mathbf{u})$ denotes the energy cost of formation of a cluster of size $n$, velocity $\mathbf{u}$ and mass $m(n)=m_{1} n$, and is given by

$$
C(n, \mathbf{u})=\Delta G(n)+\frac{1}{2} m(n) \mathbf{u}^{2},
$$

where $\Delta G(n)$ represents the energy of formation of a cluster of size $n$ at rest and the second term is its kinetic energy. By using the local equilibrium distribution (5.7), the chemical potential can be written as

$$
\mu(n, \mathbf{x}, \mathbf{u}, t)=k_{B} T \ln f(n, \mathbf{x}, \mathbf{u}, t)+C(n, \mathbf{u}),
$$

which corresponds to that of an ideal system. That is, interactions between clusters are neglected.

Our next step is to formulate the balance equations governing the evolution of the relevant quantities of the system: the probability density $f$, the internal energy $e(\mathbf{x}, t)$, and the entropy $s(\mathbf{x}, t)$. In absence of external forces, the continuity equation for $f$ may in general be written as

$$
\frac{\partial f}{\partial t}=-\mathbf{u} \cdot \nabla f-\frac{\partial}{\partial \mathbf{u}} \cdot \mathbf{J}_{u}-\frac{\partial J_{n}}{\partial n}
$$

where $J_{n}$ is the flux of clusters in size space, and $\mathbf{J}_{u}$ is a new current resulting from the interaction of the clusters with the heat bath.

The balance of local energy density can be formulated $[1,14]$ as follows

$$
\frac{\partial \rho e}{\partial t}=-\nabla \cdot \mathbf{J}_{q}
$$

which states that the total internal energy of the fluid element at $\mathbf{x}$ can only be altered by the presence of a heat flux $\mathbf{J}_{q}$, in the absence of external forces and viscous heating effects.

We can now calculate the rate of entropy change per unit of volume by differentiating the Gibbs equation (5.2) with respect to time

$$
\frac{\partial \rho s}{\partial t}=\frac{1}{T} \frac{\partial \rho e}{\partial t}-\frac{1}{T} \int \mu \frac{\partial f}{\partial t} d n d \mathbf{u}
$$


Introducing the energy balance (5.11) and the continuity equation (5.10), we obtain

$$
\begin{aligned}
& \frac{\partial \rho s}{\partial t}=-\frac{1}{T} \nabla \cdot \mathbf{J}_{q} \\
& +\frac{1}{T} \int\left(\mathbf{u} \cdot \nabla f+\frac{\partial}{\partial \mathbf{u}} \cdot \mathbf{J}_{u}+\frac{\partial J_{n}}{\partial n}\right) d n d \mathbf{u} .
\end{aligned}
$$

Integrating by parts, assuming that the fluxes vanish at the boundaries, and introducing Eq. (5.9) for the chemical potential, we can write down expression above in the form of a balance equation

$$
\frac{\partial \rho s}{\partial t}=-\nabla \cdot \mathbf{J}_{s}+\sigma
$$

where the entropy flux $\mathbf{J}_{s}$ is given by

$$
\mathbf{J}_{s}=\frac{1}{T} \mathbf{J}_{q}^{\prime}-k_{B} \int \mathbf{u} f(\ln f-1) d n d \mathbf{u}-\int f \mathbf{u} \frac{(\Delta G(n)-\Delta H(n))}{T} d n d \mathbf{u}
$$

and the entropy production $\sigma$, which must be positive semidefinite according to the second law of thermodynamics, is

$$
\sigma=-\frac{1}{T^{2}} \mathbf{J}_{q}^{\prime} \cdot \nabla T-\frac{1}{T} \int \mathbf{J}_{u} \cdot \frac{\partial \mu}{\partial \mathbf{u}} d n d \mathbf{u}-\frac{1}{T} \int J_{n} \frac{\partial \mu}{\partial n} d n d \mathbf{u} .
$$

In previous equations, $\Delta H(n)$ is the enthalpy of an $n$-cluster and $\mathbf{J}_{q}^{\prime}$ is the irreversible heat flux

$$
\mathbf{J}_{q}^{\prime}=\mathbf{J}_{q}-\int f \mathbf{u}\left(\Delta H(n)+\frac{1}{2} m(n) \mathbf{u}^{2}\right) d n d \mathbf{u}
$$

in which the integral represents the transfer of heat due to the diffusion of the clusters. In order to obtain the entropy flux we have additionally employed the identity

$$
\int \nabla \cdot(\mathbf{u} f) \ln f d n d \mathbf{u}=\nabla \cdot\left(\int \mathbf{u} f(\ln f-1) d n d \mathbf{u}\right),
$$

and the thermodynamic relation [1]

$$
\nabla\left(\frac{\Delta G(n)}{T}\right)=-\frac{\Delta H(n)}{T^{2}} \nabla T
$$

valid when the system is in mechanical equilibrium. 
The entropy production can be interpreted as a sum of products between forces-fluxes pairs. In this particular situation, we may identify from equation (5.16) the thermodynamic forces

$$
-\frac{1}{T^{2}} \nabla T,-\frac{1}{T} \frac{\partial \mu}{\partial \mathbf{u}},-\frac{1}{T} \frac{\partial \mu}{\partial n},
$$

conjugated to the heat $\mathbf{J}_{q}$, velocity $\mathbf{J}_{u}$, and size $J_{n}$ fluxes, respectively. It becomes then clear that the origin of the thermodynamic forces are the presence of gradients or, in accordance with Eq. (5.6), variations of the distribution function with respect to its local equilibrium value.

Now, according to the tenets of nonequilibrium thermodynamics, we may postulate linear phenomenological relations between thermodynamic forces and fluxes. Assuming isotropy, implying that currents and forces of different tensorial nature are not coupled, and locality in the internal space since physical currents at each point $(n, \mathbf{u})$ of the internal space are only determined by the local properties, one obtains

$$
\begin{gathered}
\mathbf{J}_{q}^{\prime}=-\frac{L_{T T}}{T^{2}} \nabla T-\int \frac{1}{T} L_{T u} \cdot \frac{\partial \mu}{\partial \mathbf{u}} d n d \mathbf{u} \\
\mathbf{J}_{u}=-\frac{L_{u T}}{T^{2}} \nabla T-\frac{1}{T} L_{u u} \cdot \frac{\partial \mu}{\partial \mathbf{u}} \\
J_{n}=-\frac{1}{T} L_{n n} \frac{\partial \mu}{\partial n}
\end{gathered}
$$

where the phenomenological coefficients satisfy the Onsager reciprocal relation

$$
L_{T u}=-L_{u T}
$$

It is useful to redefine the phenomenological coefficients in a more convenient way. Defining $\lambda \equiv \frac{L_{T T}}{T^{2}}$ as the thermal conductivity, $D_{n} \equiv \frac{k_{B} L_{n n}}{f}$ as the diffusion coefficient in $n$-space, and $\beta \equiv \frac{m L_{u u}}{f T}$ and $\xi \equiv \frac{L_{u T}}{f T}$ as friction coefficients, and using the expression for the chemical potential (5.9), the currents can be written as follows

$$
\begin{gathered}
\mathbf{J}_{q}^{\prime}=-\lambda \nabla T+\int \xi m\left(f \mathbf{u}+\frac{k_{B} T}{m} \frac{\partial f}{\partial \mathbf{u}}\right) d n d \mathbf{u}, \\
\mathbf{J}_{u}=-\xi \frac{f}{T} \nabla T-\beta\left(f \mathbf{u}+\frac{k_{B} T}{m} \frac{\partial f}{\partial \mathbf{u}}\right),
\end{gathered}
$$




$$
J_{n}=-D_{n}\left(\frac{\partial f}{\partial n}+\frac{1}{k_{B} T} \frac{\partial C}{\partial n} f\right) .
$$

Introducing now these expressions into the continuity equation (5.10), we finally obtain the Fokker-Planck equation

$$
\begin{aligned}
& \frac{\partial f}{\partial t}=-\mathbf{u} \cdot \nabla f+\frac{\partial}{\partial n}\left[D_{n}\left(\frac{\partial f}{\partial n}+\frac{1}{k_{B} T} \frac{\partial C}{\partial n} f\right)\right]+ \\
& +\frac{\partial}{\partial \mathbf{u}} \cdot\left[\beta\left(f \mathbf{u}+\frac{k_{B} T}{m} \frac{\partial f}{\partial \mathbf{u}}\right)\right]+\frac{\partial}{\partial \mathbf{u}} \cdot\left(\frac{\xi}{T} f \nabla T\right),
\end{aligned}
$$

which governs the evolution of the inhomogeneous density distribution of clusters in a bath with a non-uniform temperature distribution. The phenomenological coefficients $\beta$ and $\xi$ still remain unspecified. Their identification must be carried out through a proper interpretation of the macroscopic relaxation equations derived from the Fokker-Planck equation. This point will be discussed in the next section.

\subsubsection{Homogeneous Nucleation in Spatially Inhomogeneous Systems. Diffusion Regime}

The Fokker-Planck equation (5.28) provides the complete description of the evolution of the probability distribution of the clusters at mesoscopic level. In particular, it can even describe inertial regimes at the earlier stages of the nucleation process and retains information about the velocity of the clusters. However, the process of equilibration in velocity space is usually much faster than the remaining ones. After times much larger than the characteristic time for the relaxation of the velocity, the system enters the diffusion and thermal diffusion regime for which the evolution is governed by a simpler set of equations. In this section, we will discuss the main features of this diffusion regime.

After equilibration in velocity space, for which its distribution achieves its Maxwellian equilibrium form, the velocity does not longer constitute a relevant variable in the kinetic description of the system. Instead, we can describe the system in terms of the moments of the distribution function, which are related to the hydrodynamic fields, namely the reduced probability density, defined as

$$
f_{c}(n, \mathbf{x}, t)=\int f(n, \mathbf{x}, \mathbf{u}, t) d \mathbf{u}
$$

the velocity in $(n, \mathbf{x})$-space 


$$
\mathbf{v}_{c}(n, \mathbf{x}, t)=f_{c}^{-1} \int \mathbf{u} f d \mathbf{u}
$$

and the second moment

$$
\mathcal{P}=\int f\left(\mathbf{u}-\mathbf{v}_{c}\right)\left(\mathbf{u}-\mathbf{v}_{c}\right) d \mathbf{u},
$$

which is related to the kinetic definition of the pressure tensor $[1,15,16]$.

The corresponding balance equations for these moments can be obtained by introducing the Fokker-Planck equation (5.28) in the definitions of these quantities, after performing the time derivative and the required integrations in velocity space $[11,17]$. The resulting evolution equations for the three first moments are, respectively, the continuity equation

$$
\frac{\partial f_{c}}{\partial t}=-\nabla \cdot f_{c} \mathbf{v}_{c}-\frac{\partial}{\partial n} \int J_{n} d \mathbf{u}
$$

the balance equation for the velocity

$$
f_{c} \frac{d \mathbf{v}_{c}}{d t}=-\nabla \cdot \mathcal{P}-\beta f_{c} \mathbf{v}_{c}-\frac{\xi}{T} f_{c} \nabla T-\int\left(\mathbf{u}-\mathbf{v}_{c}\right) \frac{\partial}{\partial n} J_{n} d \mathbf{u}
$$

and the balance equation for the pressure tensor

$$
\begin{aligned}
\frac{d}{d t} \mathcal{P}=-\nabla \cdot \mathcal{Q}-2\left(\mathcal{P} \cdot \nabla \mathbf{v}_{c}\right)^{s} & -\mathcal{P} \nabla \cdot \mathbf{v}_{c}-2 \beta \mathcal{P}-\frac{2 k_{B} T}{m} f_{c} \beta \\
& -\int\left(\mathbf{u}-\mathbf{v}_{c}\right)\left(\mathbf{u}-\mathbf{v}_{c}\right) \frac{\partial}{\partial n} J_{n} d \mathbf{u}
\end{aligned}
$$

In the previous expressions $\mathcal{Q}=\int\left(\mathbf{u}-\mathbf{v}_{c}\right)\left(\mathbf{u}-\mathbf{v}_{c}\right)\left(\mathbf{u}-\mathbf{v}_{c}\right) f d \mathbf{v}$ is related with the kinetic part of the heat flux, an upper $s$ means symmetric part of a tensor, and the total derivative is defined as

$$
\frac{d}{d t} \equiv \frac{\partial}{\partial t}+\mathbf{v}_{c} \cdot \nabla
$$

In a similar way, we could derive the evolution equations for the higher-order moments of the distribution, which constitute a coupled hierarchy of hydrodynamic equations $[11,17,18]$. Time-scale considerations will allow us to simplify this hierarchy. 
The balance equation for the velocity (5.33) reveals the forces acting over the cluster, and facilitates the identification of the phenomenological coefficients $\beta$ and $\xi$.

On one hand, the third term on the right hand side of that equation manifests a force acting on the cluster arising from the presence of the temperature gradient. The presence of this force gives rise the thermophoretic effect or thermophoresis [19]-[22], and its origin can be naively understood from microscopic basis [20]. The cluster immersed in a thermal gradient is being hit by particles of the host fluid. However, the collisions with particles of the "hot" region are stronger than the ones from "cold" side, thus generating a net force driving the particle to the cold region. Phenomenologically, the force per unit mass over a particle is given by

$$
F_{T}=-\frac{\varsigma}{m} \nabla T
$$

where $\varsigma$ is called the thermophoretic coefficient. By comparison with the third term on the right hand side of Eq. (5.33) we can relate our phenomenological coefficient $\xi$ with the thermophoretic coefficient, through the expression

$$
\xi=\varsigma T \text {. }
$$

On the other hand, the second term on the right hand side of Eq. (5.33) can be identified with the hydrodynamic force exerted by the fluid on the cluster, with $\beta$ playing the role of the friction constant. That friction constant establishes the characteristic relaxation time scale $\beta^{-1}$ for the velocity. It can be estimated through Stokes' law $\beta \simeq \frac{6 \pi \eta a}{m}$, which indicates that its magnitude is very large for small clusters, as was verified in the previous chapter. Consequently, the discussion of the behavior of the system may be carried out by expanding the hierarchy of evolution equations for the moments in powers of $\beta^{-1}$. This time scale also motivates the separation of the dynamics into two well-differentiated regimes: an inertial regime for $t \ll \beta^{-1}$, characterized for the relaxation of the variables toward the diffusion regime, which is achieved for $t \gg \beta^{-1}$.

A remarkable feature of our theory is the fact that it provides a simple and complete framework to accurately analyze the dynamics of mesoscopic systems, even at inertial regimes and very short times. The set of equations (5.32)-(5.34) which govern the hydrodynamic behavior of the 'gas' of clusters, are analogous to the ones obtained in kinetic theory [15],[16]. Notice that our continuity equation (5.10) resembles the usual Boltzmann equation, with the flux $\mathbf{J}_{u}$ acting as the collision integral. Therefore our mesoscopic theory is able to reproduce 
the results of the kinetic theory in a more simple way, inside the framework of nonequilibrium thermodynamics.

For instance, by retaining more orders in the $\beta^{-1}$ expansion of the continuity equation, one can successively recover corrections to the diffusion behavior, analogous to the Chapman-Enskog or Burnett order corrections of the Boltzmann equation in the kinetic theory [17]. It is important to highlight that corrections in orders of $\beta^{-1}$ also arise from higher order moments, which must then be included for consistency [11].

However, the short-time inertial regime is usually not observed in typical experiments, since they are focused on the nucleation phenomenon occurring on a longer time scale. Hence we shall center our description in the diffusion regime. Consequently, for our purposes, it is sufficient to truncate the hierarchy of moments at the level of the pressure tensor, discarding contributions from the heat flux and higher order moments. In addition, we shall retain only the lowest order correction in $\beta^{-1}$.

We will first discuss the diffusion approximation in the evolution equations for the pressure tensor (5.34) and the velocity (5.33).

The terms appearing in the evolution equation for the pressure tensor (5.34), involve different time scales. In the diffusion regime, that is for $t \gg \beta^{-1}$, time derivatives can be neglected when compared with terms proportional to $\beta$. Notice also that the divergence term $\nabla \cdot \mathbf{v}_{c}$ is essentially a time derivative, as follows from Eq. (5.115), and can accordingly be neglected. Moreover, velocity relaxation is usually faster than relaxation in the size-space, as was shown in the previous chapter. Consequently, we can also neglect the contribution arising from the current $J_{n}$. Taking all these considerations into account, the equation for the pressure tensor then reduces to

$$
\mathcal{P}=\frac{k_{B} T}{m} f_{c} \mathbb{1}
$$

which is the one of an ideal gas $[1,14]$ being $\mathbb{1}$ the unit tensor. Introducing the value of the pressure tensor in Eq. (5.33) for the evolution of the velocity yields

$$
f_{c} \frac{d \mathbf{v}_{c}}{d t}=-\nabla \cdot\left(D_{0} \beta f_{c} \mathbb{1}\right)-\beta \frac{\xi \beta^{-1}}{T} f_{c} \nabla T-\beta f_{c} \mathbf{v}_{c}-\int\left(\mathbf{u}-\mathbf{v}_{c}\right) \frac{\partial}{\partial n} J_{n} d \mathbf{u}
$$

where $D_{0}=\frac{k_{B} T}{m \beta}$ is the spatial diffusion coefficient. In the diffusion regime we can again safely neglect the time derivative and the term proportional to $J_{n}$. The resulting equation can be written as 


$$
\mathbf{J}_{D}=f_{c} \mathbf{v}_{c}=-\nabla\left(D_{0} f_{c}\right)-\frac{\xi \beta^{-1}}{T} \nabla T f_{c}
$$

which defines the current in the diffusion regime. Inserting previous expression in the continuity equation (5.32) yields

$$
\frac{\partial f_{c}}{\partial t}=\nabla \cdot\left(\nabla\left(D_{0} f_{c}\right)+\xi \beta^{-1} \nabla \ln T f_{c}\right)-\frac{\partial}{\partial n} \int J_{n} d \mathbf{u}
$$

where the integral of the current in $n$-space with respect to velocities is

$$
\widetilde{J}_{n} \equiv \int J_{n} d \mathbf{u}=\int\left(\frac{D_{n}}{k_{B} T} \frac{\partial C(n)}{\partial n} f+D_{n} \frac{\partial f}{\partial n}\right) d \mathbf{u}
$$

We can reasonably assume that the diffusion coefficient in $n$-space, $D_{n}$, is approximately independent of the velocity, but in general the drift term $\frac{\partial C}{\partial n}$ does depend on $\mathbf{u}$. In fact, from the energy barrier (5.8) this drift is

$$
\frac{\partial C}{\partial n}=\frac{1}{2} m_{1} u^{2}+\frac{\partial \Delta G(n)}{\partial n} .
$$

However, in the diffusion regime the system has achieved equilibration in the velocity space. Therefore the relation

$$
\int \frac{1}{2} m u^{2} f d \mathbf{u}=\frac{3}{2} k_{B} T f_{c}
$$

reminiscent of the energy equipartition law, holds as in equilibrium. The integral of the current in size-space can then be written as

$$
\begin{array}{r}
\widetilde{J}_{n}=D_{n}\left[\frac{\partial f_{c}}{\partial n}+\frac{1}{k_{B} T}\left(\frac{\partial \Delta G}{\partial n}+\frac{3}{2} \frac{k_{B} T}{n}\right) f_{c}\right] \\
=D_{n}\left[\frac{\partial f_{c}}{\partial n}+\frac{1}{k_{B} T} \frac{\partial \widetilde{\Delta G}(n)}{\partial n} f_{c}\right]
\end{array}
$$

where $\widetilde{\Delta G}(n)$ is the modified nucleation barrier whose value is

$$
\widetilde{\Delta G}(n)=\Delta G(n)+\frac{3}{2} k_{B} T \ln n,
$$

which includes in an averaged way the effects of the Brownian diffusion of clusters. Notice that that is the same correction discussed in the previous chapter. 
The density balance equation can then finally be expressed as

$$
\frac{\partial f_{c}}{\partial t}=\nabla \cdot\left(\nabla\left(D_{0} f_{c}\right)+\xi \beta^{-1} \nabla \ln T f_{c}\right)+\frac{\partial}{\partial n}\left[D_{n}\left(\frac{\partial f_{c}}{\partial n}+\frac{1}{k_{B} T} \frac{\partial \widetilde{\Delta G}}{\partial n} f_{c}\right)\right] .
$$

This is the equation governing the evolution of the cluster distribution function in spatially inhomogeneous systems in the diffusion regime.

It is convenient to rewrite the spatial flux of clusters as follows

$$
\mathbf{J}_{D}=-D_{0} \nabla f_{c}-\frac{k_{B}}{m \beta} f_{c} \nabla T-\frac{\xi \beta^{-1}}{T} f_{c} \nabla T=-D_{0} \nabla f_{c}-D_{t h} \frac{\nabla T}{T} f_{c}
$$

where it becomes evident that $J_{D}$ has two contributions: normal diffusion described by Fick's law and a drift term that can be identified with thermal diffusion. The quantity

$$
D_{t h}=\left(D_{0}+\frac{\xi}{\beta}\right)=D_{0}\left(1+\frac{\xi m}{k_{B} T}\right)
$$

is the thermal diffusion coefficient. Another interesting quantity is the thermal diffusion ratio

$$
k_{T}=\frac{D_{t h}}{D}
$$

which measure of the importance of thermal diffusion also known as Soret effect. Finally, it is important to highlight that the relation found in Ref. [22] between the thermophoresis and the Soret effect,

$$
\varsigma=k_{B}\left(k_{T}-1\right),
$$

is automatically recovered in our formalism, as follows trivially from Eqs. (5.37), (5.49) and (5.50).

\subsubsection{Temperature Evolution}

Our formalism not only gives the evolution of the probability density, but can also describe the coupled evolution of the temperature field originating from the balance of internal energy.

The local internal energy has two contributions: the internal energy of the clusters and that of the heat bath. If we neglect thermal expansion, variations of the total internal energy can be related with temperature variations through 
the thermodynamic relation $\rho \delta e=c_{V} \rho \delta T$, where $c_{V}$ is the specific heat of the system bath plus clusters at constant volume. In the nonequilibrium situation where nucleation occurs, and neglecting viscous heating effects, the total internal energy may vary due to the heat flux and also due to the release of latent heat associated with the phase transformation. The heat released in the formation of a cluster of the new stable phase of size $n$ is $\ell m(n) f_{c}$, where $\ell$ is the latent heat per unit mass. Consequently, the equation governing the evolution of the temperature field is

$$
\rho c_{V} \frac{d T}{d t}=-\nabla \cdot \mathbf{J}_{q}+\ell \frac{d \rho_{t}}{d t}
$$

where $\rho_{t}(\mathbf{x}, t)=\int m(n) f_{c} d n$ represents the total density of crystallized material.

We have seen that the coupling between thermal and diffusion effects modifies the diffusion current. In addition, it also modifies the heat flux. To show this feature it is more convenient to work with the unmodified heat current because it is the quantity usually measured in experiments

$$
\mathbf{J}_{q}=\mathbf{J}_{q}^{\prime}+\int \mathbf{u}\left(\Delta H(n)+\frac{1}{2} m u^{2}\right) f d n d \mathbf{u} .
$$

Inserting in this expression the phenomenological equation for the flux (5.25) and using the result

$$
\int \mathbf{u} \frac{1}{2} m u^{2} f d n d \mathbf{u}=\int k_{B} T \mathbf{J}_{D} d n
$$

valid in the diffusion regime, we obtain

$$
\mathbf{J}_{q}=-\lambda \nabla T+\int\left(\Delta H(n)+k_{B} T+\xi m\right) \mathbf{J}_{D} d n .
$$

Now, employing Eq. (5.48) for the diffusion current $\mathbf{J}_{D}$, we finally arrive at

$$
\mathbf{J}_{q}=-\tilde{\lambda} \nabla T-\int D_{t h} k_{B} T \frac{\nabla f_{c}}{f_{c}} d n+\int \Delta H(n) \mathbf{J}_{D} d n
$$

where

$$
\widetilde{\lambda}=\lambda+\int \frac{D_{t h}^{2} k_{B} T}{D f_{c}} d n
$$

is the modified heat conductivity.

Finally, the equation for the evolution of the temperature is then 


$$
c_{V} \rho \frac{d T}{d t}=\nabla \cdot(\widetilde{\lambda} \nabla T)-\nabla \cdot \int \Delta H(n) \mathbf{J}_{D} d n+\nabla \cdot \int D_{t h} k_{B} T \frac{\nabla f_{c}}{f_{c}} d n+\ell \frac{d \rho_{t}}{d t}
$$

The former expression enables one to identify the basic mechanisms responsible for temperature variations: heat conduction, convection, thermal diffusion effects, and the release of latent heat in the crystallization process, corresponding to the different terms on the right hand side of that equation, respectively. Notice that, by neglecting the contributions arising from the diffusion of clusters and the modification of the heat conductivity, one can recover the usual equation for the evolution of temperature field

$$
\rho_{t} c_{v} \frac{d T}{d t}=\lambda \nabla^{2} T+\ell \frac{d \rho_{t}}{d t} .
$$

Once developed the general formalism describing nucleation in inhomogeneous media, our aim now will be to analyze the direct influence that the presence of these inhomogeneities may play in the nucleation process. That is the topic of the next section.

\subsubsection{Influence of Diffusion and Thermal Diffusion in Nucleation Experiments}

\section{Condensation Experiments in Thermal Diffusion Cloud Chambers}

Many systems in which nucleation occurs are spatially nonuniform. In fact, some experimental setups impose and take advantage of these inhomogeneities to bring about and measure nucleation rates. For instance, as we mentioned in the first chapter, in diffusion experiments a temperature (thermal diffusion chambers) or a velocity gradient (laminar flow diffusion chambers) is imposed to generate a very narrow region in which supersaturation exceeds the critical value and thus nucleation occurs.

Different mechanism exists through which the presence of inhomogeneities may influence the nucleation process. On one hand, gradients change the heat and mass transport in the system. We have also shown that the transport coefficients may be altered by the presence of gradients. Moreover, thermal and diffusion effects may induce convection, which strongly alters the transport in the chamber. In that case, the supersaturation and temperature conditions under which nucleation occurs may not be accurately described on these experiments. This is an important problem which has been profusely studied in the literature [23]-[28], and will not be discussed here. 


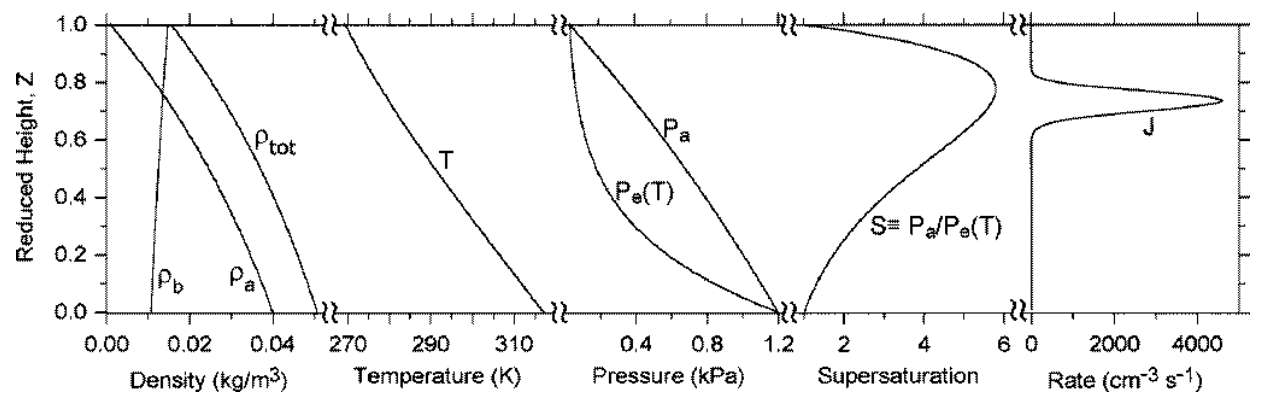

Figure 5.1: Typical profiles of total gas density $\rho_{t o t}$, temperature $T$, equilibrium vapor pressure $p_{e q}(T)$, supersaturation $S$, and nucleation rate $J$, in a thermal diffusion cloud chamber (taken from Ref. [30]).

On the other hand, there is a direct effect of loss of sub-critical clusters due to diffusion and thermal diffusion. If the region in which nucleation occurs is narrow, sub-critical clusters may escape from that region before having time to grow beyond the critical size. This mechanism may prevent nucleation or reduce its rate significantly. We will focus our analysis on this effect.

In real experiments in thermal diffusion cloud chambers, the imposition of a temperature gradient generates a temperature, pressure and supersaturation profiles as the ones depicted in Fig. 5.1. Measurements are performed under steady state conditions, thus implying that these profiles and the nucleation rate are stationary. As one can see in Fig. 5.1, the region in which nucleation takes place is quite narrow. The activated nature of nucleation implies that when supersaturation is below the critical value, nucleation is practically completely prevented.

Under these conditions, one can simplify Eq. (5.47) describing homogeneous nucleation in spatially inhomogeneous systems [29]. We can then reasonably assume that nucleation takes place only in a small region of thickness $d_{0}$ (see Fig. 5.2).

The loss of sub-critical clusters per unit of volume in that region by diffusion and thermal diffusion can be approximated by

$$
\frac{1}{A d_{0}} \int \nabla \cdot J_{D} d \mathbf{x} \simeq \frac{J_{D}}{d_{0}}=\frac{1}{d_{0}}\left(-D_{0} \nabla f_{c}-D_{t h} \frac{\nabla T}{T} f_{c}\right) .
$$

The saturation ratio decays rapidly on both sides, and due to the fact that the concentration of clusters strongly depends on supersaturation, there is virtually no cluster outside that region. If the concentration of clusters is zero on the boundaries of the chamber, then one can estimate the gradients for diffusion 


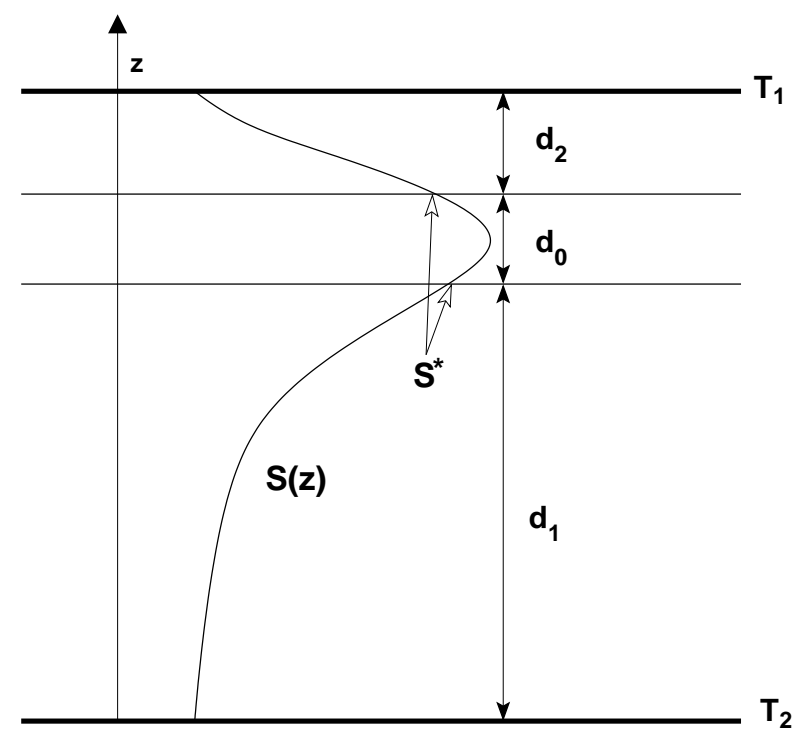

Figure 5.2: Schematic supersaturation profile in a diffusion cloud chamber.

as $f_{c} / d_{1}$ and $f_{c} / d_{2}$. Taking these considerations into account the rate of loss of $n$-sized clusters by diffusion and by thermal diffusion is

$$
-\frac{1}{d_{0} d} D_{0} f_{c}-D_{t h} \nabla \ln T \frac{1}{d_{0}} f_{c}
$$

where $d^{-1}=d_{1}^{-1}+d_{2}^{-1}$.

It is important to remark that these approximations for the diffusion and thermal diffusion terms of Eq. (5.47) are lower bounds that underestimate the actual value, since a sub-critical cluster will decompose immediately outside the region $d_{0}$. Introducing these simplifications and the stationary condition into Eq. (5.47), we obtain

$$
0=-\frac{\partial}{\partial n} \widetilde{J}_{n}-D_{0} \frac{1}{d d_{0}} f_{c}-D_{t h} \frac{1}{d_{0}} \frac{d \ln T}{d z} f_{c}
$$

where $\widetilde{J}_{n}$ is the flux of clusters in size space.

Since only order of magnitude estimates of diffusion and thermal diffusion effects are of interest, we will perform some additional simplifications. For simplicity, we will not include the nonequilibrium correction to the nucleation barrier given by Eq. (5.46), which certainly modifies the actual value of the nucleation rate, but has a weak influence in the alterations due to diffusion, 
because its logarithmic dependency on the size. We will then adopt the CNT model for the flux of clusters

$$
\widetilde{J_{n}}=-k^{+}(n) f_{e q} \frac{\partial}{\partial n}\left(\frac{f}{f_{e q}}\right)
$$

where $f_{e q}(z, t)$ is the equilibrium distribution of clusters given by

$$
f_{e q}(z, t)=N_{1}(t) \exp \left(n \ln S-\theta n^{2 / 3}\right),
$$

with $N_{1}$ being the total number of monomers.

It is also convenient to construct a dimensionless version of equation (5.62) resorting to time-scale considerations. It is evident that the effect of diffusion will be important when the time that the cluster takes to diffuse out of the nucleation region is smaller than the time the cluster takes to grow beyond the critical size. The spatial and thermal diffusion coefficients, and the rate of growing of a cluster provide the proper time scales for each one of these processes. The characteristic nucleation relaxation time (see previous chapter and Ref. [31]) is

$$
\tau_{n}=\frac{\delta^{2}}{2 k^{+}\left(n^{*}\right)}
$$

where $\delta=3\left(n^{*}\right)^{2 / 3} \theta^{-1 / 2}$ defines the width of the interval around the critical size $n^{*}=\left(\frac{2 \theta}{3 \ln S}\right)^{3}$ where the height of the nucleation barrier has drop $1 k_{B} T$. The diffusion and thermal diffusion time scales are in turn

$$
\tau_{B}=\frac{d d_{0}}{D_{0}\left(n^{*}\right)}
$$

and

$$
\tau_{t h}=\frac{d_{0}}{\frac{d \ln T}{d z} D_{t h}}
$$

respectively. Therefore, the dimensionless parameters which control the influence of diffusion and thermal diffusion effects are

$$
a=\frac{\tau_{n}}{\tau_{B}}
$$

and

$$
b=\frac{\tau_{n}}{\tau_{t h}} .
$$

It is then clear, that a value of $a$ or $b$ bigger than one implies that the time required for a cluster to nucleate is bigger than the time it takes to diffuse away 
of the nucleating region $d_{0}$ by diffusion or thermal diffusion, respectively. In that case a reduction in the nucleation rate by loss of sub-critical clusters is expected. Contrarily, values of $a$ and $b$ smaller than one mean that a cluster in the nucleation region has enough time to become stable, growing beyond the critical size, before it diffuses away. In that situation, the nucleation rate is not significantly altered by diffusion or thermal diffusion.

Introducing these parameters into Eq. (5.62), and defining the reduced distribution function $y \equiv f / f_{e q}$, and size $x \equiv n / n^{*}$, we obtain the dimensionless expression

$$
\varepsilon^{2} \frac{d^{2} y}{d x^{2}}+\left(\frac{2}{3 x} \varepsilon^{2}+6\left(1-x^{-1 / 3}\right)\right) \frac{d y}{d x}-2\left(a x^{-4 / 3}+b x^{-2 / 3}\right) y=0
$$

where $\varepsilon \equiv \delta / n^{*}$. This equation is useful to estimate the effects of the inhomogeneities in the nucleation rate for different values of the parameters $a$ and $b$.

\section{Numerical Estimates for the Case of Condensation}

To analyze the importance of diffusion and thermal diffusion in real nucleation experiments, we have solved numerically, using Mathematica [32], the equation (5.70) for different values of the parameters $a$ and $b$. We have employed the usual boundary conditions $y=1$ for $n=1$, and $y=0$ for $n \rightarrow \infty$, that for practical reasons has been chosen as $x=3$.

For the case of condensation, the expression of the diffusion and thermal diffusion coefficients can be borrowed from the kinetic theory. In fact, the Brownian diffusion coefficient of a $n$-sized cluster is approximately given by [15]

$$
D_{0}(n)=\frac{12 \pi\left(k_{B} T\right)^{3 / 2} n^{-2 / 3}}{8 P_{t o t} \sqrt{2 \pi M_{c}} s_{1}}
$$

where $P_{t o t}$ is the total pressure and $M_{c}$ is the molecular mass of the carrier gas. The thermal diffusion coefficient can be approximated [15] by

$$
D_{t h}=k_{T} D(n)=\alpha_{T} x_{1}\left(1-x_{1}\right) D(n),
$$

where $\alpha_{T}$ is the thermal diffusion factor, and $x_{1}=p / P_{t o t}$ is the mole fraction of the nucleating substance. One then obtains 


$$
a=\frac{27 \pi\left(k_{B} T\right)^{3}}{4 p_{e q} S P_{t o t} s_{1}^{3} \sqrt{\frac{M_{c}}{m_{1}}}} \phi
$$

and

$$
b=\frac{27 \pi\left(k_{B} T\right)^{3}}{4 p_{e q} S P_{t o t} s_{1}^{3} \sqrt{\frac{M_{c}}{m_{1}}}} \alpha_{T} x_{1}\left(1-x_{1}\right) \psi,
$$

where $\phi=\left(d d_{0}\right)^{-1}$ and $\psi=\frac{d \ln T}{d z} d_{0}^{-1}$. In the previous expressions, the parameters which can be changed significantly are the total pressure, and specially the equilibrium (coexistence) pressure which decreases exponentially with the temperature. One can then infer that pressure is the main property controlling the importance of diffusion and thermal diffusion is the pressure. At low enough pressures, $a$ and $b$ can become of the order of unity, thus indicating the potential relevance of diffusion and thermal diffusion under rarefied conditions.

To be more precise, we will discuss two particular situations. The first one is the influence of diffusion and thermal diffusion in a real experiment [30] in a thermal diffusion cloud chamber, using n-pentanol as a nucleating substance. And the second is a model compound mimicking the properties of a typical sulfinic acid, a substance of great interest in atmospheric processes. Thermophysical properties of both substances are listed in Table 5.1. Typical values of the geometric parameters in a diffusion cloud chamber are [33]: $d_{0}=0.1 h$, $d_{1}=0.7 h$, and $d_{2}=0.2 h$, where $h=42.3 \mathrm{~mm}$ is the height of the chamber, which results in $\phi=3.6$, and $\psi \sim 0.1$ for a representative value of the difference between the temperature of the upper and lower plates of $50 \mathrm{~K}$.

Figures 5.3 and 5.4 represent the ratio between the rate of nucleation in the presence of diffusion and thermal diffusion effects and that in its absence, as a function of the dimensionless parameters $a$ and $b$. Plots have been obtained from numerical resolution of Eq. (5.70) using Mathematica, and the properties for n-pentanol at $T=260 \mathrm{~K}, S=9$, and $P_{t o t}=30 \mathrm{kPa}$ [30].

The estimated values of the dimensionless parameters $a$ and $b$ corresponding to that experimental conditions are $a=510^{-8}$ and $b=810^{-12}$. As one can observe from Figs. 5.3 and 5.4, these small values of $a$ and $b$ imply that neither diffusion not thermal diffusion have a relevant influence in the results of these particular experiments. That fact is mainly due to the high values of both the equilibrium pressure and the total pressure under which these experiments are performed.

The situation changes drastically when either equilibrium pressure of the 
Table 5.1: Thermophysical properties of n-pentanol and the model compound using helium as a carrier gas. The properties are: $M$, the molar weight; $p_{e q}$, the saturation pressure; $\rho_{l}$, the liquid density; $\sigma$, the surface tension; $M_{c}$, the molar weight of the carrier gas (helium); $\alpha_{T}$, is the thermal diffusion factor; and $x_{1}$ is the mole fraction. In the table, $T$ represents the absolute temperature in Kelvins and $Z=1-T / 588.15$. Data taken from Refs. [29] and [30].

n-pentanol

$$
\begin{aligned}
& M=88.15 \mathrm{~g} / \mathrm{mole} \\
& p_{\text {eq }}=133.322 \exp (90.079043-9788.384 / T-9.9 \log T) P a \\
& \rho_{l}=0.270+1.930229 Z^{1 / 3}-8.414762 Z^{2 / 3}+19.226001 Z-18.559303 Z^{4 / 3} \\
& \quad+6.555718 Z^{5 / 3} \mathrm{~g} / \mathrm{cm}^{3} \\
& \sigma=26.85469-0.07889(T-273.15) \mathrm{dyn} / \mathrm{cm} \\
& M_{c}=4.0026 \mathrm{~g} / \text { mole } \\
& 1 / \alpha_{T}=(-0.7272-T /(16.36-0.2882 T))\left(x_{1}+0.12281\right)+0.089303
\end{aligned}
$$

Model compound

$$
\begin{aligned}
& M=94.13 \mathrm{~g} / \mathrm{mole} \\
& \rho_{l}=0.868 \mathrm{~g} / \mathrm{cm}^{3}\left(v_{1}=1.810^{-3} \mathrm{~cm}^{3}\right) \\
& \sigma=30.0 \mathrm{dyn} / \mathrm{cm} \\
& p_{\text {eq }}=10^{-5} \text { Torr }
\end{aligned}
$$

substance or the total pressure are low. To illustrate that fact, we have repeated the previous calculation using now the model compound whose properties are listed in Tab. 5.1. Figures 5.5 and 5.6 illustrate the influence of diffusion and thermal diffusion effects on the nucleation rate for this model compound at $T=293.15 K, S=12.5[29,31]$. When nucleation takes place at normal pressure, $P_{t o t}=1 \mathrm{~atm}$, the resulting value for the parameter controlling the diffusion effects is $a=10^{-4} \phi$, indicating again that diffusion is not specially relevant. However, this model compound presents the peculiarity of having a small saturation pressure. That fact indicates that if the total pressure is low enough, diffusion effects can be important. In fact, in experiments performed in thermal diffusion chambers, for stability reasons the chamber must be operated at reduced pressure to avoid convection [26, 27]. Using a realistic bound of $P_{\text {tot }}<100 p_{e q}$, one obtains that $a>3$ for $P_{t o t}<10^{-2}$ Torr, thus implying a reduction of the nucleation rate by three orders of magnitude, as evidenced by Fig. 5.5. This fact confirms that diffusion may have a significant influence in 


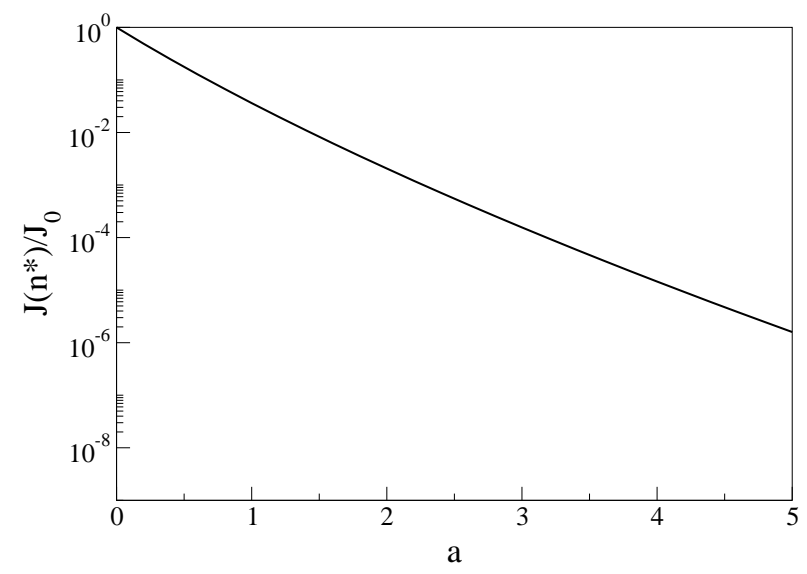

Figure 5.3: Ratio between the rate of nucleation with diffusion, $J\left(n^{*}\right)$, and without diffusion, $J_{0}$, for n-pentanol at $T=260 K, S=9$, as a function of the dimensionless parameter $a$ (and $b=0)$.

the nucleation rate of substances at reduced pressures.

The properties some pollutants commonly found at the atmosphere are similar to the ones of our model compound [29]. Therefore, the results of this section provide a hint about the relevant implications that diffusion effects may have in atmospheric processes.

\section{Condensation Experiments in Laminar Flow Diffusion Cloud Cham- bers}

The previous analysis remains valid for experiments performed in laminar flow diffusion cloud chambers [34]-[36]. In these experiments, the imposition of a steady laminar flow is responsible for the appearance of a region in which the temperature abruptly drops and nucleation occurs. We can see in Fig. 5.7 representative temperature, supersaturation and nucleation rate profiles occurring in these experimental devices. Once again, the region in which nucleation takes place is narrow, and temperature gradients are also present. Therefore, we can use the results of the previous section to infer the relevance of diffusion and thermal diffusion in this experiments.

To be more precise, let us consider a typical experiment using n-pentanol as a nucleating substance. From Ref. [36], representative conditions for this experiments are $T=260 \mathrm{~K}, S=10$, and $P_{t o t}=100 \mathrm{kPa}$. Inserting the properties of n-pentanol tabulated in Tab. (5.1) in Eqs. (6.2) and (5.74), yields the values $a=410^{-9} \phi$, and $b=810^{-12} \psi$ for the dimensionless parameters. These 


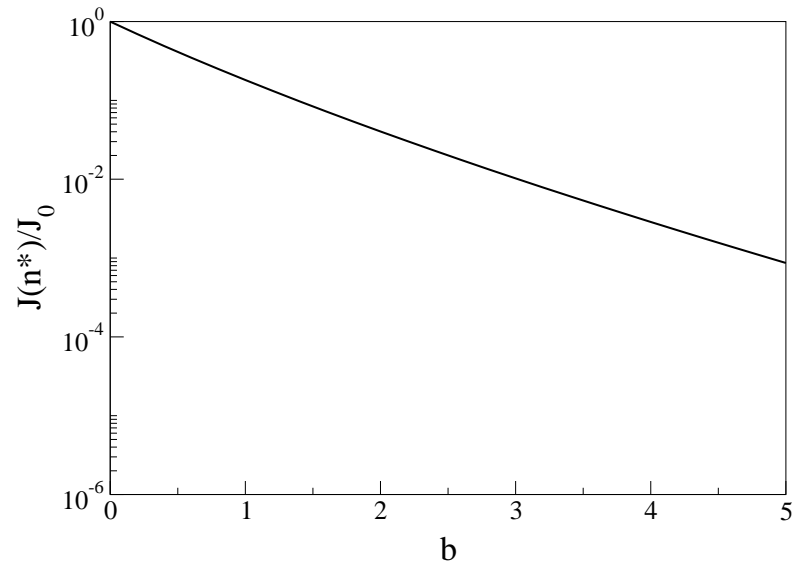

Figure 5.4: Ratio between the rate of nucleation in the presence of thermal diffusion, $J\left(n^{*}\right)$, and in its absence, $J_{0}$, for n-pentanol at $T=260 K, S=9$, as a function of the dimensionless parameter $b$ (for $a=0$ ).

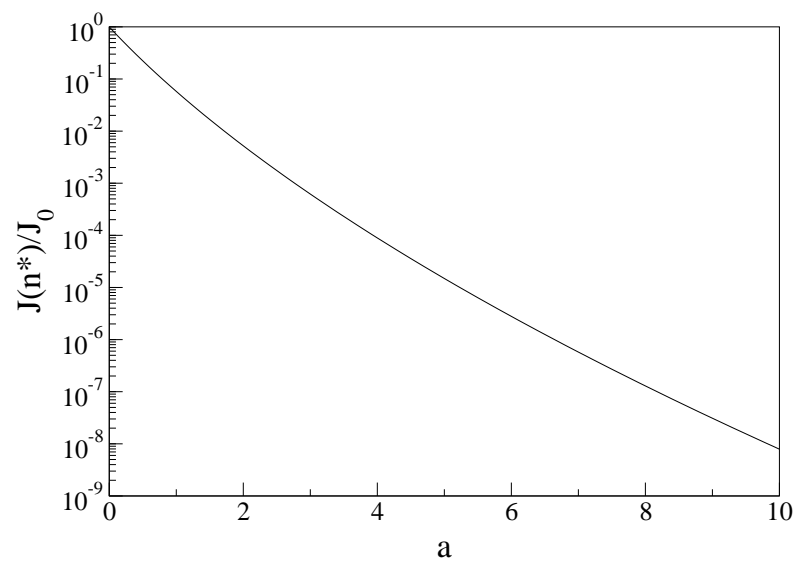

Figure 5.5: Ratio between the rate of nucleation with diffusion, $J\left(n^{*}\right)$, and without diffusion, $J_{0}$, for the model compound at different values of the dimensionless parameter $a($ and $b=0)$. 


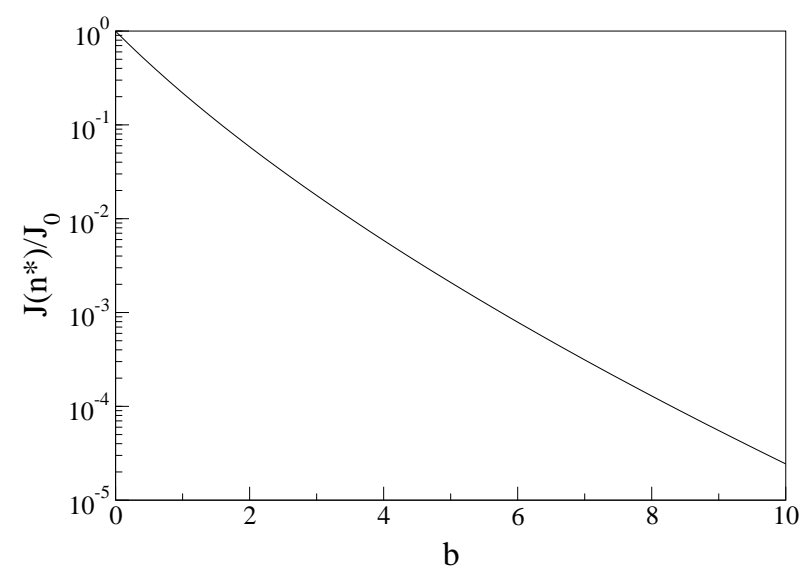

Figure 5.6: Ratio between the rate of nucleation affected by thermal diffusion, $J\left(n^{*}\right)$, and without diffusion, $J_{0}$, for the model compound at different values of the dimensionless parameter $b$ (and $a=0)$.

very small values of $a$ and $b$ evidence that, under those particular conditions, these experiments in laminar flow diffusion cloud chambers are not significantly affected by the loss of subcritical clusters by diffusion or thermal diffusion.

\section{Influence in Polymer Crystallization}

A simplified model of polymer crystallization can be carried out in terms of the coordinates of the center of mass of the polymer crystallite and the number of monomers that it contains. Under that assumptions, the analysis developed in the previous section remains valid to describe the polymer crystallization process.

Although the theoretical description of condensation and crystallization can be similar, the crystallization process presents some peculiarities that makes its description more difficult, as discussed in Chapter 1. For the case of crystallization, and specially for polymer crystallization, the identification of the transport coefficients is not so straightforward. Moreover, as discussed in the first chapter, the attachment rate $k^{+}(n)$ and specially the surface tension are not well known. That is the reason why, it is very difficult to perform a detailed quantitative estimation of the diffusion and thermal diffusion effects in the case of polymer crystallization. Nevertheless, we will present here a qualitative discussion of the potential influence of these effects.

As we mentioned in Chapter 1, in the case of crystallization the forward rate constant $k^{+}(n)$ is roughly proportional to the diffusion, as established by 


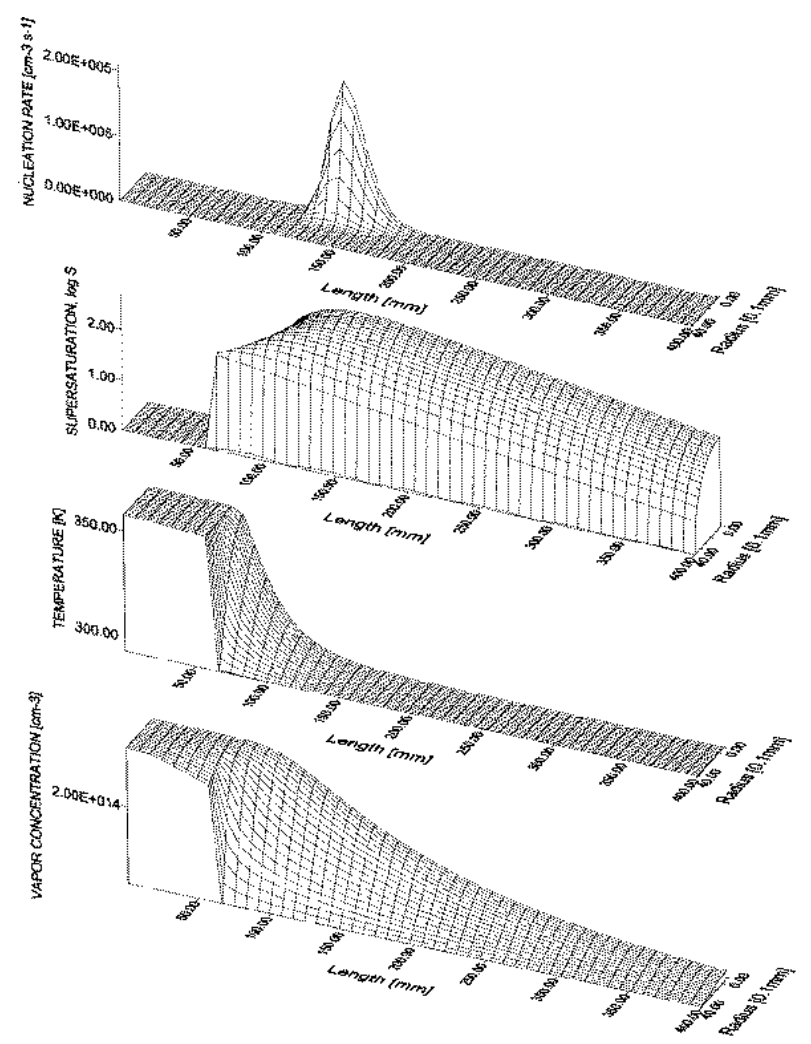

Figure 5.7: Typical profiles of nucleation rate, supersaturation, temperature and vapor concentration inside a laminar flow diffusion cloud chamber (taken from Ref. [35]).

Eq. (1.24). This proportionality implies that any modification of diffusion in the system alters the crystallization rate accordingly, since the mechanism of diffusion is also responsible for the growth of the crystals. The relevance of that fact becomes evident for the case of crystallization under nonisothermal conditions. When a temperature gradient is present in the system, the diffusivity of monomers is changed by the appearance of the thermal diffusion mechanism, as stated by Eq. (5.48). Moreover, the total diffusivity is no longer isotropic but is different in the direction dictated by the gradient. Since any reduction or increase of the diffusivity leads to the corresponding reduction /increase of the nucleation rate, this fact implies that the presence of temperature gradients may significantly alter the nucleation rate. Let us estimate the potential magnitude of this effect.

Thermal diffusion is proportional to the temperature gradient and to the 
thermal diffusion coefficient $D_{t h}$, as manifested by Eq. (5.48). This former coefficient is in general smaller than normal diffusion in gases, but may become extremely relevant in polymers. A measure of the importance of thermal diffusion effects follows from the ratio between thermal and diffusion coefficients, called Soret coefficient

$$
S=\frac{D_{t h}}{D_{0} T x_{1}\left(1-x_{1}\right)}
$$

Typical values of this coefficient for gaseous and liquid mixtures range from $10^{-3}$ to $10^{-5} K^{-1}$ in orders of magnitude, which indicates that mass diffusion is dominant. For polymer solutions, however, the situation is completely differ-

ent. The Soret coefficient increases with the molecular weight to the extent of becoming significant at high values of the molecular weight, as reported in Ref. [37]. Moreover, the small thermal conductivity of the melted polymer provoke that the release of latent heat involved in the crystallization process sets up very big temperature gradients, as follows from Eq. (5.59). Both factors imply that the magnitude of the thermal diffusion in polymers under non-isothermal conditions is relevant, and therefore can influence very importantly the crystallization process.

\subsubsection{Conclusions}

In summary, the conclusions derived from our analysis are that condensation in thermal and laminar flow diffusion cloud chambers is not significantly affected by diffusion and thermal diffusion effects, when experiments are performed at normal conditions. However, in rarefied media, as in upper atmosphere or for substances with low equilibrium vapor pressures, it can become extremely relevant.

Another situation in which the nucleation process can be drastically influenced by the presence of a temperature gradient is the case of polymer crystallization. Precisely, polymers use to have low thermal conductivities, which set up very big gradients, and high values of the Soret coefficient. Since both factors control the relevance of thermal diffusion, this is a signature that this effects may become crucial in real crystallization.

Finally, it is worth to mention that diffusion and thermal diffusion effects have proved to be also important in chemical vapor deposition, as analyzed in Ref. [31]. 


\subsection{Nucleation in a Shear Flow}

Another interesting case in which the presence of the medium may exert an influence on the nucleation process arises from the presence of flows or, in general, stresses in the system. This factor is specially relevant in crystallization, which often involves mechanical processing of the melt, such as extrusion, shearing or injection. This feature may have drastic consequences in the crystallization process. Therefore it would be desirable to build up a model that can take into account these mechanical influences in a consistent and proper way. Several models have been proposed to capture some features of phase transitions under shear flows [38] but up to now, there is no theory providing a complete description of the crystallization process in that conditions.

Our objective in this section will be to analyze the potential effects of mechanical stresses in nucleation and crystallization, focusing on the simplest influence. To that end, we will discuss the case of nucleation in the presence of a stationary flow, which for simplicity we will choose a shear flow.

It is worth pointing out that the presence of the flow breaks down the isotropy of the system, distinguishing between diffusion in different directions. Moreover, it introduces spatial inhomogeneities, which induce spatial, velocity and temperature fluxes. Cross effects between these currents may be in general very important, and consequently the evolution of the probability density, velocity and temperature fields will be governed by a highly coupled set of kinetic differential equations. These equations can be derived within our general framework, following the steps developed in the previous sections, but the resulting equations could be quite complex. For illustrative purposes, it is better to restrict our analysis to effects purely originated from the presence of the flow. Consequently, for the sake of simplicity, we will assume isothermal conditions.

The system we consider is the metastable phase in which the emerging clusters (liquid droplets or crystallites) are embedded. We assume that this metastable phase acts as a heath bath imposing a constant temperature.

Following the scheme developed in previous sections, we will first formulate the Gibbs equation for the entropy variations of this system with respect to the reference state. In this particular situation, our reference state is a stationary state characterized by the steady velocity profile of the shear flow $\mathbf{v}_{0}(\mathbf{x})$.

Assuming local equilibrium, entropy variations are given through the Gibbs equation (see Appendix)

$$
T \rho \delta s=\rho \delta e+p \rho \delta \rho^{-1}-\rho \int \mu \delta c d n d \mathbf{u}
$$


Here $s(\mathbf{x}, t)$ and $e(\mathbf{x}, t)$ are the local entropy and energy per cluster, respectively; $p(\mathbf{x}, t)$ is the hydrostatic pressure, $\rho(\mathbf{x}, t)$ is the total number of clusters per unit of volume, $\mu(n, \mathbf{x}, \mathbf{u}, t)$ is the non-equilibrium chemical potential, and $c(n, \mathbf{x}, \mathbf{u}, t)=f(n, \mathbf{x}, \mathbf{u}, t) / \rho$ is the number fraction of clusters. The former equation remains valid for changes in time and position into a mass element followed along the center of gravity motion of the clusters 'gas' [1]. The explicit expression for the chemical potential can again be obtained by comparison of the Gibbs equation (5.76) with the Gibbs' entropy postulate, yielding

$$
\mu(n, \mathbf{u}, \mathbf{x}, t)=k_{B} T \ln \frac{f}{f_{l e q}}+\mu_{l e q}(\mathbf{x})
$$

where $\mu_{\text {leq }}(\mathbf{x})$ denotes the local equilibrium chemical potential, which is independent of the internal variables, and $f_{l e q}$ is the local equilibrium distribution function corresponding to the reference state whose expression is again

$$
f_{l e q}(n, \mathbf{u}, \mathbf{x})=\exp \left(\frac{\mu_{l e q}-C(n, \mathbf{u})}{k_{B} T}\right) .
$$

The quantity $C(n, \mathbf{u})$ denotes the energy cost of formation of a state described by the internal variables $(n, \mathbf{u}$,$) , given now by$

$$
C(n, \mathbf{u})=\Delta G(n)+\frac{1}{2} m(n)\left(\mathbf{u}-\mathbf{v}_{0}\right)^{2},
$$

where $\Delta G(n)$ represents again the energy of formation of a cluster of size $n$ at rest and the second term is its kinetic energy with respect to the steady state velocity profile $\mathbf{v}_{0}$.

The next step toward obtaining the Fokker-Planck equation, is the formulation of the conservation laws for the 'gas' of clusters. In the absence of external body forces, the distribution function obeys the continuity equation

$$
\frac{\partial f}{\partial t}=-\mathbf{u} \cdot \nabla f=-\frac{\partial}{\partial \mathbf{u}} \cdot \mathbf{J}_{u}-\frac{\partial J_{n}}{\partial n}
$$

which introduces the current $\mathbf{J}_{u}$ in phase space, arising from the interaction of the clusters with the metastable phase.

In order to derive the entropy balance equation, we also need the expression for the variations of the energy with respect to its value at the stationary state. The presence of the external flow is responsible for the appearance in that equation of the term of 'viscous heating', giving rise to variations in the temperature field. To maintain isothermal conditions, and following previous ideas introduced in the implementation of the so-called 'homogeneous shear' 
$[39,40]$, we will assume the existence of a local heat source capable to remove the heat generated in the process. Under this assumption, the energy of the volume elements remains constant along their motion and its balance equation can be omitted in the subsequent analysis.

Introducing then the equation for number fraction

$$
\rho \frac{d c}{d t}=-\nabla \cdot f\left(\mathbf{u}-\mathbf{v}_{0}\right)-\frac{\partial}{\partial \mathbf{u}} \cdot \mathbf{J}_{u}-\frac{\partial J_{n}}{\partial n}
$$

derived in the Appendix, into the Gibbs equation (5.76), we obtain after some straightforward calculations the entropy balance equation

$$
\rho \frac{d s}{d t}=-\nabla \cdot \mathbf{J}_{s}+\sigma
$$

where the entropy flux $\mathbf{J}_{s}$ is given by

$$
\mathbf{J}_{s}=-k_{B} \int f(\ln f-1)\left(\mathbf{u}-\mathbf{v}_{0}\right) d n d \mathbf{u}-\frac{1}{T} \int C(n, \mathbf{u}) f\left(\mathbf{u}-\mathbf{v}_{0}\right)^{2} d n d \mathbf{u}
$$

and the entropy production, $\sigma$, which must be positive according to the second law of thermodynamics, is

$$
\begin{array}{r}
\sigma=-\frac{1}{T} \int \mathbf{J}_{u} \cdot \frac{\partial \mu}{\partial \mathbf{u}} d n d \mathbf{u}-\frac{1}{T} \int J_{n} \frac{\partial \mu}{\partial n} d n d \mathbf{u} \\
-\frac{1}{T} \int f\left(\mathbf{u}-\mathbf{v}_{0}\right) \cdot \nabla\left(\frac{1}{2} m(n)\left(\mathbf{u}-\mathbf{v}_{0}\right)^{2}\right) d n d \mathbf{u} .
\end{array}
$$

In order to obtain the entropy flux we have additionally employed the identity

$$
\int \nabla \cdot\left(\left(\mathbf{u}-\mathbf{v}_{0}\right) f\right) \ln f d n d \mathbf{u}=\nabla \cdot \int\left(\mathbf{u}-\mathbf{v}_{0}\right) f(\ln f-1) d n d \mathbf{u}-\rho \nabla \cdot \mathbf{v}_{\mathbf{0}}
$$

and we have omitted in the entropy production the term $-\left(k_{B} \rho-\frac{p}{T}\right) \nabla \cdot \mathbf{v}_{0}$ due to the bulk viscosity, which is in general negligibly small.

The entropy production can be interpreted as a sum of products between forces-fluxes pairs. In this particular situation, the forces are $-\frac{1}{T} \frac{\partial \mu}{\partial \mathbf{u}},-\frac{1}{T} \frac{\partial \mu}{\partial n}$, and $-\frac{1}{T} \nabla\left(\frac{1}{2} m(n)\left(\mathbf{u}-\mathbf{v}_{0}\right)^{2}\right)$ and their conjugated fluxes are the velocity flux $\mathbf{J}_{u}$, the flux in size space $J_{n}$, and the spatial relative current $\mathbf{J}_{x} \equiv f\left(\mathbf{u}-\mathbf{v}_{0}\right)$, respectively. 
The next step is the formulation of linear phenomenological equations relating those fluxes and forces. The situation we are considering present some peculiarities. Spatial and velocity currents have both the same tensorial character (they are both vectors) which implies that in general they must be coupled. Moreover, the presence of the shear flow breaks down the isotropy of the system by introducing a privileged direction. Consequently, phenomenological coefficients are expected to be in general tensors. In addition, locality in the internal space will be assumed again. Taking all these considerations into account, the expressions for the currents are

$$
\begin{gathered}
\mathbf{J}_{x}=+\frac{m}{T} \mathcal{L}_{x x} \cdot \nabla \mathbf{v}_{0} \cdot\left(\mathbf{u}-\mathbf{v}_{0}\right)+\frac{1}{T} \mathcal{L}_{u x} \cdot \frac{\partial \mu}{\partial \mathbf{u}} \\
\mathbf{J}_{u}=-\frac{1}{T} \mathcal{L}_{u u} \cdot \frac{\partial \mu}{\partial \mathbf{u}}+\frac{m}{T} \mathcal{L}_{u x} \cdot \nabla \mathbf{v}_{0} \cdot\left(\mathbf{u}-\mathbf{v}_{0}\right) \\
J_{n}=-\frac{1}{T} L_{n n} \frac{\partial \mu}{\partial n}
\end{gathered}
$$

where we have used the Onsager reciprocal relation

$$
\mathcal{L}_{u x}=-\mathcal{L}_{x u}
$$

and the result

$$
\nabla\left(\frac{1}{2} m\left(\mathbf{u}-\mathbf{v}_{0}\right)^{2}\right)=-m \nabla \mathbf{v}_{0} \cdot\left(\mathbf{u}-\mathbf{v}_{0}\right)
$$

It is useful to redefine the phenomenological coefficients in a more convenient way. By identifying $D_{n} \equiv \frac{k_{B} L_{n n}}{f}$ as the diffusion coefficient in $n$-space, which in turn can be identified with the rate of attachment of monomers to a cluster $k^{+}(n) ; \vec{\alpha}=\frac{m \mathcal{L}_{u u}}{f T}$ and $\vec{\zeta}=\frac{m \mathcal{L}_{u x}}{f T}$ as friction tensors, and introducing the explicit form of the chemical potential, equation (5.9), the currents $\mathbf{J}_{u}$ and $J_{n}$ can be written as

$$
\begin{gathered}
\mathbf{J}_{u}=-f\left[\vec{\alpha}+\vec{\zeta} \cdot \nabla \mathbf{v}_{0}\right] \cdot\left(\mathbf{u}-\mathbf{v}_{0}\right)-\vec{\alpha} \frac{k_{B} T}{m} \cdot \frac{\partial f}{\partial \mathbf{u}} \\
J_{n}=-D_{n}\left(\frac{\partial f}{\partial n}+\frac{1}{k_{B} T} \frac{\partial C}{\partial n} f\right) .
\end{gathered}
$$

Introduction of these expressions for the currents into the continuity equation yields 


$$
\begin{array}{r}
\frac{\partial f}{\partial t}=-\mathbf{u} \cdot \nabla f+\frac{\partial}{\partial n}\left[D_{n}\left(\frac{\partial f}{\partial n}+\frac{1}{k_{B} T} \frac{\partial C}{\partial n} f\right)\right]+ \\
+\frac{\partial}{\partial \mathbf{u}} \cdot\left(\left[\vec{\alpha}+\vec{\zeta} \cdot \nabla \mathbf{v}_{0}\right] \cdot f\left(\mathbf{u}-\mathbf{v}_{0}\right)+\vec{\alpha} \frac{k_{B} T}{m} \cdot \frac{\partial f}{\partial \mathbf{u}}\right)
\end{array}
$$

which corresponds to the Fokker-Planck equation describing the evolution of the inhomogeneous density distribution of clusters in the presence of a steady state flow.

\subsubsection{Balance Equations in the Diffusion Regime}

The Fokker-Planck equation (5.93) we have derived retains information about the evolution of the cluster velocity distribution. Although the movement of clusters may play a significant role in the nucleation process, as we verified in the previous chapter, velocity dependencies can hardly be measured because velocity distribution relaxes to equilibrium very fast. Therefore, as discussed in Section 5.1.1, we can perform a simplified description of the process. The relevant quantities will then be the distribution function for the density of clusters together with the velocity in $n$-space defined in equations (5.29) and (5.30).

Proceeding along the lines of Section 5.1.1, integration of Eq. (5.93) over velocities leads to the corresponding balance equations for the density of clusters in n-space

$$
\frac{\partial f_{c}}{\partial t}=-\nabla \cdot f_{c} \mathbf{v}_{c}-\frac{\partial}{\partial n} \int J_{n} d \mathbf{u}
$$

for the velocity

$$
f_{c} \frac{d \mathbf{v}_{c}}{d t}=-\nabla \cdot \mathcal{P}-\mathcal{B} \cdot f_{c}\left(\mathbf{v}_{c}-\mathbf{v}_{0}\right)-\int\left(\mathbf{u}-\mathbf{v}_{c}\right) \frac{\partial}{\partial n} J_{n} d \mathbf{u}
$$

and for the reduced pressure tensor

$$
\begin{gathered}
\frac{d}{d t} \mathcal{P}=-\nabla \cdot \mathcal{Q}-2\left(\mathcal{P} \cdot \nabla \mathbf{v}_{c}\right)^{s}-\mathcal{P} \nabla \cdot \mathbf{v}_{c}-2(\mathcal{B} \cdot \mathcal{P})^{s}-\frac{2 k_{B} T}{m} f_{c}(\rightrightarrows) \\
-\int\left(\mathbf{u}-\mathbf{v}_{c}\right)\left(\mathbf{u}-\mathbf{v}_{c}\right) \frac{\partial}{\partial n} J_{n} d \mathbf{u}
\end{gathered}
$$

where $\mathcal{Q}$ is the kinetic part of the heat flux and $\mathcal{B} \equiv \vec{\alpha}+\vec{\zeta} \cdot \nabla \mathbf{v}_{0}$ 
A remarkable feature is the fact that the balance equation for the pressure tensor Eq. (5.96) is similar to the one obtained for the thirteen moment approximation to the Boltzmann equation [16]. Once again our mesoscopic nonequilibrium thermodynamic treatment of the problem has been able to recover results similar results to that of kinetic theory. In particular, inertial regimes and relaxation equations for the main quantities involved can be obtained inside this framework. This result reinforce the fact that application of the well-established non-equilibrium thermodynamics postulates as indicated in [1], suffices to provide a general scheme under which non-equilibrium processes of macroscopic and mesoscopic nature can be treated.

The second term on the right hand side of the velocity balance equation can be identified with the friction force per unit mass exerted on the suspended cluster by the host fluid, where $\mathcal{B}$ plays the role of the friction tensor. The modulus of this tensor determines again the characteristic time scale for the relaxation of the velocities. In general, such friction tensor can be calculated from hydrodynamics; however, for the sake of simplicity, let us approximate this tensor by the Stokes diagonal friction $\mathcal{B} \simeq \beta \mathbb{1}$. This identification leads to $\overrightarrow{\vec{\alpha}}=\beta \mathbb{1}-\overrightarrow{\vec{\zeta}} \cdot \nabla \mathbf{v}_{0}$

The inverse of the friction $\beta^{-1}$ separates again the dynamics of the system into a short-time inertial regime and the diffusion regime. For times $t>\beta^{-1}$ the system achieves the diffusion regime. In this situation, we can neglect inertial effects manifested through the presence of the time derivative $\frac{d \mathbf{v}_{c}}{d t}$. Moreover, we can neglect the contribution arising from the current $J_{n}$ in the velocity relaxation since the rate of relaxation of velocities is usually faster than the relaxation of cluster sizes determined by $D_{n}$.

Proceeding along the lines indicated in Section 5.1.1 and in Ref. [11], we find that in the diffusion regime, the expression for the pressure tensor when a shear flow is present, is

$$
\mathcal{P}=\frac{k_{B} T}{m} f_{c}\left[\mathbb{1}-\left(\beta^{-1}(\mathbb{1}+\vec{\zeta}) \cdot \nabla \mathbf{v}_{0}\right)^{s}\right]
$$

From this equation, we can conclude that Brownian motion of the particles contributes to the total pressure tensor of the suspension in two forms. The first contribution is the well-known scalar kinetic pressure given by

$$
p=\frac{k_{B} T}{m} f_{c}
$$

which is the equation of state for the ideal Brownian 'gas'. The second contribution comes from the irreversible part $\mathbf{\Pi}^{\mathbf{0}}$ of the pressure tensor, which can be 
written in the form

$$
\mathbf{\Pi}^{\mathbf{0}}=-D_{0} f_{c}\left[(\mathbb{1}+\vec{\zeta}) \cdot \nabla \mathbf{v}_{0}\right]^{0}
$$

where $D_{0}=\frac{k_{B} T}{m \beta}$ is the diffusion coefficient of the cluster when the liquid is at rest, and the superindex 0 means symmetric traceless tensor. This last equation defines the Brownian viscosity tensor

$$
\overrightarrow{\vec{\eta}}_{B}=D_{0} f_{c}(\mathbb{1}+\vec{\zeta})
$$

which contains the "Brownian viscosity" $D_{0} f_{c}$ [41], and the contribution due to the coupling with the non-equilibrium bath which is proportional to $\vec{\zeta}$.

Inserting the expression for the pressure tensor in the velocity balance equation (5.95), and taking the diffusion limit we obtain for the current of clusters

$$
\mathbf{J}_{D}=f_{c} \mathbf{v}_{c}=-\mathcal{D} \cdot \nabla f_{c}+f_{c} \mathbf{v}_{0}
$$

where

$$
\mathcal{D}=D_{0}\left[\mathbb{1}-\left(\beta^{-1}(\mathbb{1}+\vec{\zeta}) \cdot \nabla \mathbf{v}_{0}\right)^{0}\right]
$$

can be identified with the spatial diffusion coefficient, which in this situation possesses tensorial character. Using Eqs. (5.100) and (5.98), it can be rewritten as

$$
\mathcal{D}=D_{0}\left[\mathbb{1}-\left(\frac{\vec{\eta}_{B}}{p} \cdot \nabla \mathbf{v}_{0}\right)^{0}\right]
$$

Inserting this expression in the equation for the density (5.94) one finally obtains

$$
\frac{\partial f_{c}}{\partial t}=-\nabla \cdot\left(f_{c} \mathbf{v}_{0}\right)+\nabla \cdot\left(\mathcal{D} \cdot \nabla f_{c}\right)+\frac{\partial}{\partial n}\left(D_{n} \frac{\partial f_{c}}{\partial n}+\frac{D_{n}}{k_{B} T} \frac{\partial \widetilde{\Delta G}}{\partial n} f_{c}\right)
$$

which is the kinetic equation for nucleation in presence of a shear flow in the diffusion regime, and where, as discussed in Section 5.1.1, $\widetilde{\Delta G}(n)$ is the effective nucleation barrier given in the diffusion regime by Eq. (5.46). 


\subsubsection{Influence of the Shear Flow in Nucleation and Crystallization}

The goal in this subsection will be to analyze the conditions under which the presence of a mechanical stress in the system, modeled through a shear flow, may influence the nucleation process. Obviously, a shear rate modifies the transport in the system, setting up alterations in the density, pressure and temperature profiles. We will not study this heat and mass transfer problem, which is fairly complicate. Instead, we will focus on the direct effect of the presence of a flow in the nucleation rate, both in condensation and in crystallization, with especial emphasis on polymer crystallization.

\section{Influence in Condensation}

As shown in the previous section, one important influence that the flow may exert in the nucleation process is through the alteration of the diffusion given by Eq. (5.103). This modification depends on the shear rate, the pressure and the viscosity, and its magnitude is accordingly expected to be important when

$$
\frac{\eta}{p}\left|\nabla \mathbf{v}_{0}\right| \geq 1
$$

that is, for high enough values of the shear rate and low pressures.

To be more precise, let us estimate the magnitude of the correction for a typical experiment in a laminar flow diffusion cloud chamber [36]. Representative values of the shear rates, the pressure and the viscosity in these experiments are $\nabla v_{0} \sim 10^{3} \mathrm{~s}^{-1}, p \sim 10^{4} \mathrm{dyn} / \mathrm{cm}^{2}$, and $\eta \sim 10^{-4}$ poise. These numbers yield an approximate value of the order of magnitude of the correction introduced by the shear of $10^{-5}$. Consequently, in this range of values, not the diffusion coefficient nor the nucleation rate measured in that experiments are significantly altered by the shear rate.

\section{Influence in Polymer Crystallization}

Whereas in condensation under normal conditions, the modification of the diffusion coefficient due to the shear flow is in general negligible, that is not the case in crystallization. The relevance of this modification lies again in the fact that the rate of attachment of monomers to the crystal $k^{+}$and consequently the exponential prefactor of the nucleation rate, Eq. (1.26), strongly depends on the diffusivity of monomers [42]. The fact that the diffusion coefficient could be altered by the flow implies that the nucleation rate is accordingly modified. 
Moreover, the symmetry breaking inherent to the presence of the flow promotes that the diffusion coefficient is no longer a scalar quantity, but has tensorial nature. The rate of the process will depend on the direction thus making the nucleation and specially the growth of crystals no longer isotropic. Finally, the viscosity of a supercooled liquid can be very large, specially near the glass transition. In that situation, the correction of the diffusion coefficient given by Eq. (5.103) can become crucial and could modify dramatically the nucleation rate.

Another important factor one has to consider is that the flow also induces a stress acting on the surface of the cluster which may break up the droplets when they become large enough $[43,44]$. To incorporate this effect, one must include the additional energetic cost associated to the shear stress in the free energy of cluster formation.

Consequently, our results may constitute a hint to assess the relevance of the presence of a flow in crystallization.

\subsubsection{Conclusions}

The main effects that the presence of a shear flow exerts on the nucleation process can be summarized as follows. On one hand, the flow alters the transport and consequently the evolution of the growing clusters distribution function, which has implications in the effective nucleation and growth rate. On the other hand, the presence of a shear flow changes the spatial diffusion coefficient of the clusters, as shown in equation (5.102). Typical values of the parameters controlling this correction imply that this effect is not very important for condensation. However, the high viscosity and the peculiarities of polymer crystallization suggest that the presence of a shear flow may promote drastic changes in the process. The formalism we have developed in this section could then share some light to explain the experimental results of polymer crystallization under shear flow [45], although the formulation of quantitative predictions is limited by the unavailability of the values of the physical parameters required in the calculations.

As stated previously, the main objective of the section has been to analyze the effects that the flow by itself may induce on the nucleation process. That is the reason why we have assumed isothermal conditions. A more complete and realistic treatment may be carried out incorporating thermal effects, in the way discussed in the preceding section. 


\section{Appendix}

\section{Local Thermodynamic Relations}

Our purpose in this Appendix is to show the derivation of the local thermodynamic relations which have been employed in the contents of this chapter.

The Gibbs equation for a multicomponent thermodynamic system is

$$
T \delta S=\delta E+p \delta V-\sum \mu \delta N .
$$

The continuous analogous to that expression can be formulated as follows

$$
T \delta S=\delta E+p \delta V-\int \mu \delta N(n, \mathbf{u}) d n d \mathbf{u},
$$

in which different values of the variables $n$ and $\mathbf{u}$ denote different "chemical species", and $N(n, \mathbf{u})$ is the total number of particles of each specie.

Let us now construct the local version of the previous equation. To that end, we will describe the system in terms of $f(n, \mathbf{x}, \mathbf{u}, t)$, the number of cluster of size $n \in(n, n+d n)$ at $\mathbf{x} \in(\mathbf{x}, \mathbf{x}+d \mathbf{x})$, with velocity $\mathbf{u} \in(\mathbf{u}, \mathbf{u}+d \mathbf{u})$, at time $t$. We can then define the corresponding specific thermodynamic quantities $s$, $e, v$, and $c$ as follows

$$
\begin{gathered}
S=\int s \rho d \mathbf{x} \\
E=\int e \rho d \mathbf{x} \\
V=\int 1 d \mathbf{x}=\int v \rho d \mathbf{x} \\
N(n, \mathbf{u})=\int f d \mathbf{x}=\int c \rho d \mathbf{x}
\end{gathered}
$$

where $\rho(\mathbf{x}, t)=\int f d n d \mathbf{u}$ is the number density of clusters per unit volume, $v=\rho^{-1}$, and $c=\frac{f}{\rho}$.

Using these specific quantities, the local expression of the Gibbs equation is then

$$
T \delta(\rho s)=\delta(\rho e)+p \delta(1)-\int \mu \delta f d n d \mathbf{u},
$$

which is the form used in Section 5.1.

This equation can alternatively be written as 
$T \rho \delta s-\rho \delta e-p \rho \delta \rho^{-1}+\rho \int \mu \delta c d n d \mathbf{u}+\left(T s-e-p \rho^{-1}+\int \mu c d n d \mathbf{u}\right) \delta \rho=0$

which, using the definition of the Gibbs energy to cancel the last term, reduces to

$$
T \rho \delta s=\rho \delta e+p \rho \delta \rho^{-1}-\rho \int \mu \delta c d n d \mathbf{u} .
$$

That is the expression employed in Section 5.2.

\section{Balance Equation for the Number Fraction}

Integration of Eq. (5.80) with respect to the cluster velocity $\mathbf{u}$ and size, leads to the macroscopic equation of continuity, which can be written in the form

$$
\frac{d \rho}{d t}=-\rho \nabla \cdot \mathbf{v}
$$

where $\rho(\mathbf{x}, t)$ is the density of the Brownian 'gas', given by

$$
\rho(\mathbf{x}, t)=\int f d n d \mathbf{u},
$$

$\mathbf{v}(\mathbf{x}, t)$ is the average velocity of the Brownian particles defined through the expression

$$
\rho \mathbf{v}(\mathbf{x}, t)=\int \mathbf{u} f d n d \mathbf{u},
$$

and we have defined the total derivative as

$$
\frac{d}{d t} \equiv \frac{\partial}{\partial t}+\mathbf{v} \cdot \nabla
$$

Using the continuity equation (5.80) and Eq. (5.115), one obtains the equation for the number fraction

$$
\rho \frac{d c}{d t}=\frac{d f}{d t}-\frac{f}{\rho} \frac{d \rho}{d t}=-\nabla \cdot f(\mathbf{u}-\mathbf{v})-\frac{\partial}{\partial \mathbf{u}} \cdot \mathbf{J}_{u}-\frac{\partial J_{n}}{\partial n}
$$

Under the local equilibrium assumption, the average velocity $\mathbf{v}$ coincides with the velocity of the reference state, that is $\mathbf{v}=\mathbf{v}_{0}$, thus obtaining Eq. (5.81). 


\section{Bibliography}

[1] S.R. de Groot and P. Mazur, Non - Equilibrium Thermodynamics, (Dover, New York, 1984).

[2] I. Prigogine and P. Mazur, Physica XIX, 241 (1953).

[3] A. Pérez-Madrid, J.M. Rubí, and P. Mazur, Physica A 212, 231 (1994).

[4] I. Pagonabarraga, A. Pérez-Madrid, and J.M. Rubí, Physica A 237, 205 (1997).

[5] J. M. Rubí and P. Mazur, Physica A 250, 253 (1998).

[6] J. M. Rubí and A. Pérez-Madrid, Physica A 264, 492 (1999).

[7] P. Mazur, Physica A 261, 451 (1998).

[8] P. Mazur, Physica A 274, 491 (1999).

[9] J.M. Rubí and P. Mazur, Physica A 276, 477 (2000).

[10] D. Reguera, J.M. Rubí, and A. Pérez-Madrid, Physica A 259, 10 (1998).

[11] I. Santamaría-Holek, D. Reguera, and J.M. Rubí, Phys. Rev. E 63, 051106 (2001).

[12] D. Reguera, J.M. Rubí, and L.L. Bonilla, Chapter 3 in Mathematical Modelling for Polymer Processing, (V. Capasso Ed., Springer-Verlag, in press).

[13] L.D. Landau and E.M. Lifshitz, Course of Theoretical Physics Vol 5 (Statistical Physics Part 1) and Vol 9 (Statistical Physics Part 2), (Pergamon Press, New York, 1980).

[14] A. Pérez-Madrid, J.M. Rubí, and P. Mazur, Physica A 212, 231 (1994). 
[15] S. Chapman and T.G. Cowling, The Mathematical Theory of NonUniform Gases, (Cambridge University Press, Cambridge, 1970).

[16] J.O. Hirschfelder, C.F. Curtiss, and R.B. Bird, Molecular Theory of Gases and Liquids (Wiley, New York, 1954).

[17] J.M. Rubí and A. Pérez-Madrid, Physica A 264, 492 (1999).

[18] G. Wilemski, J. Stat. Phys. 14, 153 (1976).

[19] J. Tyndall, Proc. R. Inst. G. B. 6, 3 (1870).

[20] A. Einstein, Z. Phys. 27, 1 (1927).

[21] S. Chapman, Philos. Mag. 5, 630 (1928).

[22] I. Goldhirsch and D. Ronis, Phys. Rev. A 27, 1616 (1983); and 27, 1635 (1983).

[23] R.H. Heist, M. Janjua, and J. Ahmed, J. Phys. Chem. 98, 4443 (1994).

[24] J.A. Fisk and J.L. Katz, J. Chem. Phys. 104, 8649 (1996).

[25] J.A. Fisk, V.M. Chakarov, and J.L. Katz, J. Chem. Phys. 104, 8657 (1996).

[26] J.L. Katz, J. Chem. Phys. 52, 4733 (1970).

[27] A. Bertelsmann and R.H. Heist, J. Chem. Phys. 106, 610 (1997); and 106, 624 (1997) .

[28] D.Kane, S.P. Fisenko, M. Rusyniak, and M.S. El-Shall, J. Chem. Phys. 111, 8496 (1999).

[29] C. Becker and H. Reiss, J. Chem. Phys 65, 2066 (1976).

[30] M.M. Rudek, J.L. Katz, I.V. Vidensky, V. Zdímal, and J. Smolík, J. Chem. Phys. 111, 3623 (1999).

[31] G. Shi, J.H. Seinfeld, and K. Okuyama, J. Appl. Phys. 68, 4550 (1990).

[32] Mathematica(C) 4, Wolfram Research (1999).

[33] C-H. Hung, M.J. Krasnopoler, and J.L. Katz, J. Chem. Phys. 90, 1856 (1989). 
[34] K. Hämeri and M. Kulmala, J. Chem. Phys. 105, 7696 (1996).

[35] V.B. Mikheev, N.S. Laulainen, S.E. Barlow, M. Knott, and I.J. Ford, J. Chem. Phys. 113, 3704 (2000).

[36] H. Lihavainen, Y. Viisanen, and M. Kulmala, J. Chem. Phys. 114, 10031 (2001).

[37] K.J. Zhang et al., J. Chem. Phys 111, 2270 (1999).

[38] For a review see: A. Onuki, J. Phys. Cond. Matt. 9, 6119 (1997).

[39] W.T. Ashurst and W.G. Hoover, Phys. Rev. A 11, 658 (1975).

[40] E. Peakock-Lopez and J. Keizer, Phys. Lett. 108, 85 (1985).

[41] K.F. Freed and M. Muthukumar, J. Chem. Phys. 69, 2657 (1978).

[42] K.F. Kelton, A.L. Greer, and C.V. Thompson, J. Chem. Phys. 79, 6261 (1983).

[43] K.Y. Min and W.I. Goldburg, Phys. Rev. Lett. 71, 569 (1993).

[44] S. Butler and P. Harrowell, Phys. Rev. E 52, 6424 (1995).

[45] G. Eder and H. Janeschitz-Kriegl, Processing of Polymers, Chapter 5, (VCH, Germany, 1997). 


\section{Chapter 6}

\section{Crystallization Beyond Nucleation}

The content of the previous chapters have been mainly devoted to the analysis of the first step in the phase transformation, the nucleation process, focused essentially on the particular situation of condensation. The next stage in the dynamics of the phase transformation is the growth of the nucleated embryos to fill the whole space of the sample.

It is important to mention that the same formalism employed to describe nucleation remains valid for the initial steps of growth, as we will justify later on. However, at more advanced stages of the process, additional mechanisms arise, and a different description must be adopted.

The importance of a proper description of the growth stage becomes more relevant in crystallization than in condensation. The reason is that the evolution of crystallization process strongly dictates the final microstructure achieved by the solid, which in turn determine its mechanical and physical properties.

Our purpose in this chapter is precisely to sketch how the phase transition proceeds beyond the nucleation step. In the next stage, the most important process to describe is the filling of the space available by the growing of the different nucleated clusters. We will outline how the formalism we have developed in the previous chapters can be implemented in the subsequent stages of the phase transformation process, to describe this space filling. To this purpose we shall use a very simplified model of polymer crystallization.

In spite of the striking differences between crystallization of polymers and that of simple substances, polymer crystallization theories originate largely from theories developed earlier for simple substances [1]. The real description of the crystallization process of polymers at advanced stages involve complicated con- 
cepts, such as the secondary crystallization, the crystal thickening or the molecular fractioning which go beyond the scope of this chapter. A more realistic description of the growth of crystalline polymers is provided, for instance, by the approaches of Lauritzen and Hoffman [2], or Sadler and Gilmer [3], which will not be discussed here.

Nevertheless, polymers are useful models for the study of crystallization for different reasons. The first one is related to time scale considerations. An ordinary ionic substance will crystallize very rapidly from the melt once the first seed appears, because ions usually diffuse very rapidly. However, polymer molecules move slowly enough that the crystallization process can be reasonably monitored before the crystals have grown to macroscopic dimensions. On the other side, some polymers crystallize in a spherical structure known as spherulite, which permits a simplified description in terms of a single parameter describing the size of the crystal. Finally, crystallization of polymers is of interest in their own right, due to their significant relevance in industrial applications. Its importance arises from the fact that mechanical properties of any crystal polymer are determined by its morphology and internal structure, which in turn is dictated by the crystallization kinetics. For all these reasons, mathematical modeling aiming to describe and control the kinetics of polymer crystallization has achieved great interest (see for instance Refs. [4]-[8]).

One of these theories is the general Avrami-Kolmogoroff formalism, which provides a stochastic geometrical model for the development and space-filling of the new phase. The underlying physical ingredients of this theory are the nucleation and growth rates, i.e. the rate of appearance and growth of the new crystalline phase. This fact highlight the importance of developing a theory for nucleation and growth as realistic as possible. The general formalism of nucleation we have introduced in the previous chapters may then facilitate a more complete and accurate description of the crystallization process. In fact, since our formalism accounts for the influences of the host fluid in the nucleation rate, its implementation may constitute the first step toward a more realistic description of crystallization.

This chapter has been organized in the following manner. In Section 1, we shall describe the different steps underlying the polymer crystallization process. In the Section 2, we will derive a the kinetic equation suitable to describe a nonspherical cluster, which is one of the distinctive features of crystallization. In Sections 3 and 4 , in order to describe the advanced stages of the crystallization, we will use our results in the classical Avrami-Kolmogoroff model, to analyze the behavior of the crystallinity. Further extensions and improvements will be outlined in Section 5, whereas the last section is intended as a brief summary. 


\subsection{The Polymer Crystallization Process}

Crystallization of polymers is the process of structural reorganization of a melted polymer leading to the appearance of an ordered structure. In practice, crystallization proceeds by the melting and subsequent cooling down of the sample. In general, this process strongly depends on temperature. For temperatures higher than the melting temperature $T_{m}$, thermal agitation breaks up any ordered structure and the polymer remains melted in a "liquid-like" phase. Contrarily, for temperatures lower than the melting temperature, the thermodynamic stable phase is the crystalline one. The transition from melted to crystalline polymer is what is known as crystallization. This process involves diffusion of monomer units toward the crystal front. However, the diffusion mechanisms are prevented at temperatures below the glass transition temperature $T_{g}$. Consequently, crystallization takes place for temperatures in the range $T_{g}<T<T_{m}$.

For temperatures lower than $T_{m}$ crystals are formed from the melted phase by the nucleation mechanism. Density fluctuations in the liquid phase may eventually create small aggregates of polymers having the same properties than the crystalline phase. The small crystals are continuously being created and destroyed by fluctuations. The reason for the break up of the crystals is that, although below $T_{m}$ the crystal phase is the thermodynamic stable one, the formation of a crystal involves the creation of one or more interfaces between the liquid and the crystal, with the corresponding energetic cost due to the surface tension. However, as described in the case of condensation, beyond the critical size the volume effects dominate over the surface ones, and the growth of the cluster is favored by a global reduction of the energy.

Nucleation is then the initial step in the crystallization process, for it determines the appearance of the first crystal nuclei which are the germ of the second stage of crystallization process: the growth. At this stage, nuclei larger than the critical size, tend to grow through the addition of monomer units or alternatively acting as sites of heterogeneous nucleation (nucleation on the surface of the growing crystal).

Crystals grow freely until they progressively begin to compete to fill the whole space. They may eventually hit each other, and therefore the growth is stopped at the contact surface. This phenomenon, called impingement, is relevant at later stages of the process and determines the final morphology of the system.

In the final stage of crystallization, the impingement impedes the subsequent growth of the crystals. Therefore, amorphous non-crystallized matter remains trapped among clusters. Moreover, clusters themselves are not fully 
crystalline, but may contain some amorphous inclusions. This trapped material can eventually self-organize, joining to the main crystal structure and therefore increasing the crystallinity of the sample. This process of reorganization is what it is normally referred to as secondary crystallization or perfection.

In summary, we can schematically distinguish between three stages in the entire crystallization process:

- Nucleation, which is the process of formation of the initial crystalline embryos.

- Growth, which describes the space-filling of this crystals which is limited by impingement.

- Secondary crystallization, accounting for the reorganization of the amorphous inclusions to achieve a more crystalline material.

What we call a "crystallized polymer" is in fact a crystal aggregate with amorphous inclusions.

\subsection{Nucleation of Polyhedrical Clusters}

Although in the case of condensation the hypothesis of an spherical droplet is at least quite reasonable, for the case of crystallization, the potential presence of ordered structures often involves the creation of different oriented surfaces, which in turn may have distinct values of the surface tension. Therefore, in the general situation of a non-spherical anisotropic clusters, additional geometrical parameters have to be considered in order to perform an accurate description of the clusters. Several ways exist to describe the size and shape of the clusters. One plausible approach, previously employed Ref. [9], consist of describing the cluster as a polyhedrical object of volume $v$ with $N$ faces of areas $s_{1}, s_{2}, \ldots, s_{N}$. The complete description of the cluster is thus provided by the $3 \mathrm{~N}$-dimensional vector $\underline{\mathbf{R}}$

$$
\underline{\mathbf{R}}=\left\{\vec{r}_{1}, \vec{r}_{2}, \ldots, \vec{r}_{N}\right\}
$$

whose components are the $N$ vectors $\overrightarrow{r_{i}}$ normal to the individual faces $s_{i}$.

According to equation (1.15) the minimum work of formation of this polyhedrical cluster is

$$
\Delta G(\underline{\mathbf{R}})=v \Delta g+\sum \sigma_{i} s_{i}
$$


In order to obtain the kinetic equation following the procedure introduced in Chapter 2, we must consider $\underline{\mathbf{R}}$ as the internal coordinate. Notice that in this case, the state of the system (the cluster) is not determined by a single coordinate as in the isotropic case, but by the set of internal coordinates $\underline{\mathbf{R}}$. The extension of our method set up previously to deal with this multidimensional case is straightforward. Nucleation can be viewed now as a diffusion in the multidimensional space of cluster shapes. The Gibbs equation accounting for entropy variations related to changes in configuration in $\underline{\mathbf{R}}$-space is

$$
\delta S=-\frac{1}{T} \int \mu(\underline{\mathbf{R}}, t) \delta f(\underline{\mathbf{R}}, t) d \underline{\mathbf{R}},
$$

where $d \underline{\mathbf{R}} \equiv d \vec{r}_{1} \ldots d \vec{r}_{N}$. The corresponding entropy production is then given by

$$
\sigma=-\frac{1}{T} \int \underline{\mathbf{J}}(\underline{\mathbf{R}}, t) \cdot \frac{\partial \mu(\underline{\mathbf{R}}, t)}{\partial \underline{\mathbf{R}}} d \underline{\mathbf{R}},
$$

where the current in $\underline{\mathbf{R}}$-space $\underline{\mathbf{J}}(\underline{\mathbf{R}}, t)$ is defined through the corresponding conservation law of the probability density

$$
\frac{\partial f(\underline{\mathbf{R}}, t)}{\partial t}=-\frac{\partial}{\partial \underline{\mathbf{R}}} \cdot \underline{\mathbf{J}}(\underline{\mathbf{R}}, t)
$$

and $\mu(\underline{\mathbf{R}}, t)$ is a generalized chemical potential in $\underline{\mathbf{R}}$-space similar in form to the one introduced in the one-dimensional case by Eq. (2.7). The linear laws for the currents obtained from the entropy production (6.4) are

$$
\underline{\mathbf{J}}(\underline{\mathbf{R}}, t)=-\frac{1}{T} \underline{\underline{\mathbf{L}}} \cdot \frac{\partial \mu}{\partial \underline{\mathbf{R}}},
$$

which inserted in the conservation law (6.5) give the kinetic equation

$$
\frac{\partial f}{\partial t}=\frac{\partial}{\partial \underline{\mathbf{R}}} \cdot\left\{\underline{\underline{\mathbf{D}}}(\underline{\mathbf{R}}) \cdot \frac{\partial f}{\partial \underline{\mathbf{R}}}+\frac{1}{k_{B} T} \underline{\underline{\mathbf{D}}}(\underline{\mathbf{R}}) \cdot \frac{\partial \Delta G}{\partial \underline{\mathbf{R}}} f\right\}
$$

describing the evolution of the probability density $f(\underline{\mathbf{R}}, t)$. In this expression $\underline{\underline{\mathbf{D}}}=\frac{k_{B} \underline{\underline{\underline{\mathbf{L}}}}}{f}$ is a diffusion tensor, which accounts for the rate of directional growth, and may in general depend on the configuration in $\underline{\mathbf{R}}$-space. The existence of non-diagonal components of $\underline{\underline{\mathbf{D}}}$ may account for the simultaneous coupled growth in different directions.

If we neglect these couplings and identify the diagonal components of the diffusion-growth coefficient $\underline{\underline{\mathbf{D}}}$ with the forward rate $k^{+}$given in expression 
(1.22), one can recover the kinetic equation for the nucleation of a polyhedrical clusters proposed by Ziabicki [9],[10].

The former analysis then illustrates the application of our formalism to the case in which the characterization of the state of the system must be carried out by certain number of coordinates or degrees of freedom. However, in practice, the analysis of crystallization in terms of this multidimensional cluster is quite unwieldy. To capture the essential features of crystallization, the space-filling, a simplified description can be performed in terms of a single global parameter as described in the following section.

\subsection{Space-Filling in Terms of the Crystallinity Frac- tion}

The aim of the models we have presented up to now has been mainly the description of the mechanism of nucleation. However their validity is not restricted to nucleation process, as they may also describe the earlier stages of crystal growth. We have seen that nucleation can be considered as an activated process where a free energy barrier has to be surmounted. However, the barrier crossing process is of stochastic nature and in fact recent numerical simulations [11], [12] have revealed that this crossing is highly diffusive which means that the barrier can be surmounted in both directions. Consequently, the fact that a cluster is bigger than the critical size $n^{*}$ does not guarantee that it must grow indefinitely. Post-critical clusters, which are usually assumed as stable crystals, may eventually shrink and disappear. Therefore the diffusive nature of nucleation mechanism implies that the separation between a pure "nucleation" regime (as the formation of critical clusters) and a "growth" regime (for clusters larger than the critical size) is not sharp.

Definitively, the nucleation and the subsequent crystal growth are not intrinsically different processes since, at least in the initial stages, they obey the same underlying physical mechanism. In fact, the same ingredients appearing in phenomenological theories of nucleation namely, the free energy barrier $\Delta G$ and even the rate of attachment of monomers $k^{+}(n)$, remain well defined for sizes larger than the critical size. Therefore, they also describe the kinetics of the growth.

Consequently, the model we have introduced may describe nucleation and the initial stages of growth in the crystallization process. As most of nucleationaimed models, our theory is subjected to the following considerations:

- It applies while interactions between clusters are practically negligible. 
When clusters are large enough, the ideality assumption employed in the expression for the chemical potential fails, since interactions between clusters start to play a significant role. Interactions among clusters emerge as they try to fill the whole space.

- We have not considered the presence of secondary crystallization or perfection which is governed by a different mechanism. This assumption is justified from the fact that secondary crystallization is normally slower, and consequently the overall initial crystallization is usually determined by nucleation and growth.

- We have focused our study on bulk nucleation. Surface nucleation at the surface of the growing clusters can be categorized as heterogeneous nucleation.

- Finally, for the sake of simplicity, we have assumed that the shape of the clusters is spherical until impingement occurs. Consequently, polymorphic crystallization is not taken into account and anisotropic effects are neglected.

In previous chapters, we have modeled the initial stage of crystallization focusing on the evolution of the probability density of clusters of a given size. It remains now to describe the subsequent steps of the process.

It is interesting to realize that our formalism still retains information about the size distribution of crystals of the new phase. Sometimes, however, it is more convenient to work out with a simplified global description in terms of merely the fraction of material crystallized. Accordingly, one introduces a new macroscopic variable, the crystalline fraction or crystallinity $w$ defined as the fraction of the volume occupied by the crystal phase. The state $w=0$ thus corresponds to a totally melted polymer whereas $w=1$ would indicate complete crystallization. The latter situation can barely be achieved. As we have stated previously, the reason is that the resulting "crystal" is in fact a crystalline aggregate with amorphous inclusions of non-crystallized material which get trapped between crystal spherulites.

Let us first introduce the virtual volume fraction crystallized, $V$, defined as the fraction of volume that the nucleated clusters would occupy at time $t$ if they could grow freely in absence of impingement effects. This quantity differs from the actual fraction of volume crystallized in the fact that overlapping between clusters is allowed. In terms of the size distribution of clusters, it can be expressed as 


$$
V(\mathbf{x}, t)=\int_{n^{*}(T)}^{\infty} f_{c}(n, \mathbf{x}, t) \rho_{1} n d n
$$

where $\rho_{1}$ is the fraction of volume per monomer of the crystal phase, and $n^{*}$ is the size of the critical cluster that strongly depends on temperature.

In the initial stages of nucleation, the crystallinity coincides with the virtual volume fraction crystallized $w \cong V$, because impingement or overlapping between clusters is negligible. At more advances stages of the process, this statement becomes no longer valid since neither nucleation nor growth may take place in an already crystallized region.

Those effects, which can be viewed as interactions between clusters, are difficult to include in nucleation/growth theories to the extent that they are usually not considered. We can then wonder how is possible to describe the space-filling with theories that cannot include interactions or impingement of clusters. The most common answer is to resort to the mathematical theory of Avrami-Kolmogoroff.

\subsubsection{Avrami-Kolmogoroff Theory}

The theory of Avrami-Kolmogoroff [13],[14], based upon purely stochastic geometric grounds, aims to describe the space-filling in the crystallization kinetics.

In its formulation, the crystallization process is divided into two basic and well differentiated regimes: nucleation and deterministic growth. Nucleation is viewed as a point process in the space of the sample. That is, nuclei, which are the germ of crystallization, may emerge at random points of the system following a Poisson distribution. The reason for choosing this particular probability distribution for the appearance of nuclei is, apart from its simplicity, the fact that it is the one assuming less information. The rate at which nuclei appear per unit of volume at position $x$ at time $t$, denoted by $N(x, t)$, is referred to as the nucleation rate.

After the formation of the nuclei, it is assumed that it grows freely and deterministically with a radial rate $R(x, t)$. Free growth means that clusters are not aware of the presence of neighbor cluster and overlapping is allowed.

For illustrative purposes, we will proceed to discuss in some detail a particular case of this general theory leading to the formulation of the classical Kolmogoroff equation. This equation is derived for spatially homogeneous nucleation, which entails the additional assumptions of uniformity of the density and growth rate. Therefore this particular case assumes that crystallization starts randomly at different locations and subsequently propagates spherically 
outwards from the nucleation site. The question is then to calculate the probability $p(t)$ that at time $t$ a representative point $P$ of the sample is not covered by any spherulite.

The probability that the number of nucleation fronts which pass through a representative point $P$ up to time $t$ is exactly $c$ is given by the Poisson distribution

$$
p(c)=\frac{e^{-E} E^{c}}{c !},
$$

where $E$ is the average value of the number of passing waves, which can be identified with the virtual volume $V$. The probability that at time $t$ the point $P$ is not covered by any spherulite is then the probability that no fronts pass through $P$, that is that $c=0$

$$
p(0)=e^{-V} .
$$

This probability is equivalent to the fraction $(1-w)$ of the sample still remaining in the melted state. Taking this fact into account in the previous equation, one then finally arrives at the classical Kolmogoroff equation

$$
w(t)=1-e^{-V}
$$

which can be written in the alternative form

$$
\frac{\partial w}{\partial t}=(1-w) \frac{\partial V}{\partial t}
$$

As mentioned before, the degree of crystallinity of polymers seldom reaches the limiting value one. The solidified polymer is always semi-crystalline, due to the amorphous material trapped within the crystallization fronts. This feature can be taken into account by slightly modifying the previous equation in the form

$$
\frac{w(t)}{w_{m}}=1-e^{-V}
$$

where $w_{m}$ denotes the maximum crystallinity that can be achieved.

In this particular situation of spatially homogeneous isotropic nucleation and neglecting impingement effects with neighboring spherulites, the volume fraction that a spherulite, nucleated at time $s$, would occupy at time $t$ is given by

$$
v(t ; s)=\frac{4 \pi}{3}\left[\int_{s}^{t} R(u) d u\right]^{3}
$$


where the term within the brackets corresponds to the radius of the spherulite at time $t$. Therefore, the virtual volume fraction crystallized up to time $t$ is given by

$$
V(t)=\frac{4 \pi}{3} \int_{-\infty}^{t} N(s)\left[\int_{s}^{t} R(u) d u\right]^{3} d s .
$$

This formula manifests how the evolution of the crystallinity is fully determined by both nucleation $N(x, t)$ and growth $R(x, t)$ rates.

To calculate the degree of crystallinity of the polymer, one usually proposes particular forms of the nucleation and growth rates and uses Eq. (6.15) to calculate the virtual volume which is subsequently utilized in the Kolmogoroff equation (6.11) to obtain the desired variable. As an example, for the case of constant nucleation and growth rates $\left(N(t)=N_{c}\right.$ and $R(t)=R_{c}$ ) the crystallinity evolves as

$$
w(t)=1-e^{-\frac{\pi}{3} N_{c} R_{c}^{3} t^{4}} .
$$

Additional examples have been studied for instance in Ref. [6]. A common characteristic of all of them is that in spite of the different nucleation and growth mechanisms the crystallinity can be expressed through the general formula

$$
w(t)=1-e^{-K_{A} t^{A}}
$$

which is usually known as the Avrami equation. The parameter $A$ is referred to as the Avrami exponent. Experimental data of crystallization kinetics obtained by differential scanning calorimetry, dilatometry [15] or scattering [16] techniques can be fitted to estimate the value of this exponent.

In spite of its usefulness, the mathematical theory of Avrami-Kolmogoroff presents some drawbacks:

- It assumes that nucleation is a point process with Poissonian distribution. In spite that this assumption is the more reasonable one when no further information is available, it may be not valid under realistic conditions. For instance, the presence of inhomogeneities in the system can make Poisson distribution for the nucleation events no longer applicable.

- Nucleation seldom proceeds according to a simple single mechanism as one often assumes in Avrami theory.

- Experiments on crystallization kinetics not always can be reproduced by the Avrami equation. In this context, the value of the Avrami exponent 
strongly depends on the underlying model for nucleation and growth rates, which must be introduced a priori.

- The separation between nucleation and growth regimes is not always well defined. As we have shown, nucleation is a stochastic process of diffusive nature which causes that apparently stable nuclei can eventually shrink and even disappear. It is worth to emphasize that, better than delimiting different regimes, the critical size separates different tendencies (to grow or to shrink).

Some of these shortcomings can be avoided by using the approach discussed in the following section.

\subsection{Kinetic Equations for the Crystallinity}

In previous chapters, we have developed a general framework and discussed a set of examples to describe the evolution of the distribution of clusters under different realistic conditions. Although our formalism is restricted to the earlier stages of crystallization, its results can be used in the Avrami-Kolmogoroff theory to describe the space-filling. The improvements of our theory arise from the fact that it unifies nucleation and growth, retaining the stochastic nature of these processes, and incorporating possible influences of the fluid.

From the kinetic equations we have derived, one can evaluate the nucleation $N(t)$ and growth $R(t)$ rates, which take into account the physical mechanisms and try to reproduce the experimental conditions.

In fact, it is not necessary to calculate both rates $N(t)$, and $R(t)$ separately. It is more accurate to directly evaluate the virtual volume crystallized from the cluster size distribution function as shown in Eq. (6.8).

The expression for the virtual volume introduced in the Kolmogoroff equation (6.12) can describe the advanced stages of crystallization. In the following subsections, we will briefly illustrate the evolution of the crystallinity in some particular situations analyzed in previous chapters. For the sake of simplicity, we will analyze the kinetic equations for the variation of the virtual volume fraction with time which following equation (6.8) is given by

$$
\frac{\partial}{\partial t} V(\mathbf{x}, t)=\int_{n^{*}(T)}^{\infty} \frac{\partial f_{c}(n, \mathbf{x}, t)}{\partial t} \rho_{1} n d n+\frac{\partial n^{*}}{\partial T} \frac{\partial T}{\partial t} f_{c}\left(n^{*}, \mathbf{x}, t\right) \rho_{1} n^{*} .
$$

The first term on the right hand side accounts for nucleation and growth as described by the Fokker-Planck equation; and the last term refers to the 
appearance of stable clusters due to the change in the critical size originated from the variations of the temperature. This last effect is known as athermal nucleation.

Consideration of the different Fokker-Planck dynamics corresponding to the models discussed in the previous chapter gives rise to different types of kinetic equations for the evolution of the virtual volume fraction.

\subsubsection{Case 1: Isothermal Homogeneous Nucleation}

As mentioned in the previous chapter, a minimal model for a spherulite of crystallized polymer can be formulated in terms of a single parameter, the size of the crystal, characterized by the molecular content of the crystal or alternatively the radius of the sphere. The results derived in the previous chapter remain then applicable to this simplified model of polymer crystallization. In particular, under isothermal and homogeneous conditions, we have shown in Chapter 2, that the Fokker-Planck equation corresponding to this situation is given by

$$
\frac{\partial f}{\partial t}=\frac{\partial}{\partial n}\left\{D_{n} \frac{\partial f}{\partial n}+\frac{D_{n}}{k_{B} T} \frac{\partial \Delta G}{\partial n} f\right\}
$$

By using this result in the general expression for the evolution of the virtual volume fraction (6.18) one obtains

$$
\begin{array}{r}
\frac{\partial}{\partial t} V(t)=-\left.D_{n}^{*} \frac{\partial f}{\partial n}\right|_{n^{*}} \rho_{1} n^{*}-\int_{n^{*}}^{\infty} \rho_{1}\left(D_{n} \frac{\partial f}{\partial n}+\frac{D_{n}}{k_{B} T} \frac{\partial \Delta G}{\partial n} f\right) d n \\
+\frac{\partial n^{*}}{\partial T} \frac{\partial T}{\partial t} f\left(n^{*}, t\right) \rho_{1} n^{*}
\end{array}
$$

The right hand side of this expression contains contributions of different types. The first term corresponds to the flux of clusters of critical size due to diffusion at the top of the barrier. This term is, essentially, the nucleation rate times the volume of the critical cluster. Notice that there is no contribution arising from the drift because, by definition $\left.\frac{\partial \Delta G}{\partial n}\right|_{n^{*}}=0$. The second term corresponds to the usually called "growth regime" of the clusters. It accounts for the increase of the virtual volume due to the overall change in size of clusters bigger than the critical size. It is important to emphasize that the growth term is not deterministic. Contrarily, it retains the stochastic diffusive nature of the crystal growth. Finally, the last term takes into account the athermal nucleation. 


\subsubsection{Case 2: Spatial Inhomogeneous Crystallization}

The presence of spatial inhomogeneities requires the use of an additional coordinate, the position $x$, in our description and significantly modifies the equations governing the evolution of the virtual volume. By introducing the Fokker-Planck equation (5.41) derived in Section 5.1.1, the local evolution of the virtual volume fraction is given by

$$
\begin{gathered}
\frac{\partial}{\partial t} V(\mathbf{x}, t)= \\
-\left.D_{n}^{*} \frac{\partial f_{c}}{\partial n}\right|_{n^{*}} \rho_{1} n^{*}-\int_{n^{*}}^{\infty} \rho_{1}\left(D_{n} \frac{\partial f_{c}}{\partial n}+\frac{D_{n}}{k_{B} T} f_{c} \frac{\partial \widetilde{\Delta G}}{\partial n}\right) d n+\frac{\partial n^{*}}{\partial T} \frac{\partial T}{\partial t} f_{c}\left(n^{*}, \mathbf{x}, t\right) \rho_{1} n^{*} \\
+\nabla \cdot\left(\nabla \int D(n) f_{c} \rho_{1} n d n-\int \xi \beta^{-1} \nabla \ln T f_{c} \rho_{1} n d n\right)
\end{gathered}
$$

where the last term arises due to spatial inhomogeneities and thermal diffusion. One can roughly approximate this term as

$$
\begin{array}{r}
\nabla \cdot\left(\nabla \int D(n) f_{c} \rho_{1} n d n-\int \xi \beta^{-1} \nabla \ln T f_{c} \rho_{1} n d n\right) \simeq \\
\nabla \cdot\left(\langle D\rangle \nabla V(\mathbf{x}, t)-\left\langle\xi \beta^{-1}\right\rangle \nabla \ln T V(\mathbf{x}, t)\right)
\end{array}
$$

where $\langle D\rangle$ and $\left\langle\xi \beta^{-1}\right\rangle$ are the size-averaged spatial diffusion and thermal friction coefficient. From this expression, the spatial diffusive behavior of the evolution of the virtual volume becomes clearly manifested. To account for the global evolution of the virtual volume, an additional integration of Eq. (6.21) over the position coordinate is required.

\subsubsection{Case 3: Nucleation in a Shear Flow}

When the crystallizing system is under the influence of an external flow, its evolution is governed by the Fokker-Planck equation (5.104) derived in Section 5.2. In terms of the virtual volume fraction, its evolution is governed by

$$
\begin{array}{r}
\frac{\partial}{\partial t} V(\mathbf{x}, t)=-\left.D_{n}^{*} \frac{\partial f_{c}}{\partial n}\right|_{n^{*}} \rho_{1} n^{*}-\int_{n^{*}}^{\infty} \rho_{1}\left(D_{n} \frac{\partial f_{c}}{\partial n}+\frac{D_{n}}{k_{B} T} f_{c} \frac{\partial \widetilde{\Delta G}}{\partial n}\right) d n+ \\
+\frac{\partial n^{*}}{\partial T} \frac{\partial T}{\partial t} f_{c}\left(n^{*}, \mathbf{x}, t\right) \rho_{1} n^{*}-\nabla \cdot\left(V(\mathbf{x}, t) \mathbf{v}_{0}\right)+\nabla \cdot(\langle\mathcal{D}\rangle \nabla V(\mathbf{x}, t))
\end{array}
$$

where again the last term has been approximated employing the size-averaged spatial diffusion coefficient $\langle\mathcal{D}\rangle$. The novelties introduced by the presence of the 
flow concerns the two last terms of that expression, corresponding to convection and spatial diffusion of the local virtual volume which has tensorial character. Once again, to describe the total virtual volume, integration of Eq. (6.23) over the space variables is needed.

In the previous examples, we have shown how to formulate differential equations for the virtual volume corresponding to different situations. It becomes then clear that, in practical situations, the evolution of the crystallinity may exhibit a more complex behavior than the one resulting form the mathematical models usually introduced in the Avrami-Kolmogoroff theory. The natural extension of our analysis is to employ those equations to study the behavior of the crystallinity in realistic cases. The evolution of the crystallinity given by Eq. (6.12) would constitute a valid result to be checked with experimental data.

\subsection{Crystallization at Advanced Stages}

The situations introduced in which the theory we have presented applies do not completely describe the crystallization process. Our aim in this section is precisely to outline possible extensions of the theory with the main purpose of offering a more general framework in which actual physical situations can be studied.

As the nucleation theories formulated up to now, our theory introduces some assumptions that are only valid in the initial stages. These assumptions are in essence the following:

- The appearance of crystallization nuclei only occurs in a few points of the sample. Therefore, in the nucleation regime, it is reasonable to assume that crystal clusters constitute a dilute system. This means that we can neglect interactions between clusters, and the system behaves as ideal. One also assumes that clusters grow by addition of single monomers. In the dilute regime, the number of monomers is much higher than the number of binary, ternary... clusters and therefore one can neglect collisions between them. As clusters grow, collisions acquire relevance and the notion of dilute regime is no longer valid.

- The analysis of nucleation process is mostly focused on the evolution of the cluster size distribution function. One tends to identify the probability density of clusters of a given size with the number distribution of clusters present at the system. A conservation law similar to Eq. (2.8) is usually formulated for the evolution of this quantity. Consequently, conservation of the total number of clusters present at the system is assumed. 
Moreover, as stated in the Section 1.2.1, the kinetic equation for the distribution function is formulated within a finite range of sizes. Moreover, the loss of monomers due to the growth of clusters is not taken into account since one assumes an almost infinite number of them. However, as they grow reaching macroscopic sizes, the number of monomers (and clusters of small sizes) decreases significantly thus leading to a reduction of the crystallization rate.

These restrictions make nucleation theories not valid at advanced stages of crystallization process. This is the reason why these theories are only utilized as models for early stages of crystallization. To describe subsequent space-filling and impingement regimes, one has to use other approaches as the mathematical model of the Avrami-Kolmogoroff.

It would be interesting to extend the validity of nucleation theories to describe latest stages of crystallization process, overcoming their restrictions and retaining the physical nature of crystallization process. The restrictions of nucleation theories come mainly from two features: avoidance of interactions between clusters and conservation of the number of clusters.

In the following subsections we will precisely outline how these difficulties could be accounted for within the context of our theory.

\subsubsection{Interactions Between Clusters}

At the earlier stages of the process, nucleation and subsequent formation of clusters may be assumed to take place under ideal conditions for which clusters practically do not interact. The theory we have developed holds for this particular situation. A complete description of the process requires the knowledge of the kinetics at later times when clusters have grown to the extent of making ideal conditions inapplicable.

To accomplish for that situation, two slight modifications of the formalism we have presented can be carried out. On one hand, the interaction potential between clusters, $U$, must be taken into account in the expression of the chemical potential (for instance in Eq. (2.7)). One may equivalently introduce an activity coefficient $a$, defined as

$$
a=\exp \left(\frac{U}{k_{B} T}\right)
$$

in such a way that the chemical potential is now given by

$$
\mu=k_{B} T \ln a f+\Delta G
$$


On the other hand, the continuity equation (2.8) must be modified to account for direct interactions. With the introduction of these modifications, we could now proceed along the lines indicated in Section 5.1 to obtain the corresponding kinetic equation governing the system under the presence of interactions.

\subsubsection{Non-Conservative Equations}

In addition to the interactions among clusters, the advanced stages of crystallization are also characterized by the decrease of the crystallization rate. The reasons are that nucleation and growth cannot proceed in a region already crystallized, and that the amount of amorphous phase to crystallize progressively decreases. Whereas, the first item can be treated as interactions between clusters of the excluded volume type, the second one must be considered separately.

In order to take into account the reduction of the amorphous phase and the disappearance of small clusters, which end up incorporated to the larger ones, our formalism has to be extended to deal with "open systems". That is, our theory must consider the fact that the total number of cluster is not necessarily conserved.

The simplest way to introduce that feature, is to replace the continuity equation for the evolution of the population of clusters (for instance, Eq. (2.8) in the case of inhomogeneous nucleation) by a non-conservative one including a source/sink term. This term will be responsible for the appearance/disappearance of monomers/clusters of any size in the system.

The incorporation in our formalism of interactions between clusters and of a non-conservative equation governing the evolution of cluster population may lead to a complete description of crystallization even at latest stages, and may constitute a powerful model for crystallization alternative to the classical Avrami-Kolmogoroff formalism.

\subsubsection{Anisotropic Effects}

In the situations developed in previous chapters, the growing clusters feel the influence of possible external agents through the metastable phase in which they are embedded. For example, the kinetic equation governing nucleation in a temperature gradient, given by Eq. (5.28), differs from its isothermal counterpart, Eq. (6.19), in terms containing the influence of the melted phase. In its derivation, it is implicitly assumed that the nucleation mechanisms remains unaltered. 
In the initial stages of the crystallization, this assumption is fully justified. The length scale in which variations of the fields takes place (the gradient length scale) is significantly bigger than the typical size of the emerging clusters. Consequently, for all practical purposes, these clusters appear and growth under homogeneous conditions. When clusters grow, they can achieve sizes comparable to the gradients length scale. As a consequence, clusters undergo growing processes under inhomogeneous conditions leading to their deformation. In this context, it has been shown that clusters growing in the presence of a temperature gradient elongate in the direction of the gradient.

In order to describe this feature, we must incorporate the effect of the gradients in the kinetics coefficients which control the mechanism of nucleation and growth. In fact, in Section 5.2, we have precisely shown how the presence of a flow alters the rate of attachment of clusters, through modifications in the diffusion coefficient, leading to anisotropic behaviors.

\subsection{Conclusions}

In this chapter, we have introduced the basis of a theoretical framework for modeling crystallization processes. The theory we have presented is aimed to describe the initial stages of growth after nucleation, which play a very important role in crystallization processes. Moreover, it takes into account the stochastic nature of both nucleation and growth, offering a common treatment for both mechanisms, without introducing a sharp and somehow artificial splitting into two different regimes. We have also outlined how to extend the results obtained for nucleation and for earlier steps of growth to describe advanced stages of crystallization by invoking the classical Avrami-Kolmogoroff theory. Since the modeling we have performed of the nucleation stage is able to account for the influence of different external conditions concerning inhomogeneities, temperature gradients, or the presence of external flows, these influences, which may induce drastic changes in the process, are retained in the description of the space filling process. The counterpart of introducing a more realistic description of the crystallization process is that the solution of the corresponding equations becomes a more difficult task.

However, the ultimate goal would be to be able to describe the space-filling without using mathematical models but by implementing a physical kinetic model of crystallization valid even at the final stages of the process. We believe that our formalism, with the proper inclusion of interactions and of the no conservation aspects in the way outlined in the previous section, may become a valuable tool to describe crystallization process. 


\section{Bibliography}

[1] K.F. Kelton, Solid State Phys. 45, 75 (1991).

[2] J.I Lauritzen and J.D. Hoffman, J. Res. Nat. Bur. Std. 64A, 73 (1960).

[3] D.M. Sadler and G.H. Gilmer, Polymer 25, 1446 (1984).

[4] D. Andreucci, A. Fassano, M. Primicerio, and R. Ricci, Surv. Math. Ind. 6, 7 (1996).

[5] G. Eder and H. Janeschitz-Kriegl, Processing of Polymers, Chapter 5, (VCH, Germany, 1997).

[6] G. Eder in Macromolecular Design of Polymeric Materials, K. Hatada, T. Kitayama, O. Vogl (Eds.) (Marcel Dekker, New York, in press).

[7] M.L. Di Lorenzo and C. Silvestre, Prog. Polim. Sci. 24, 917 (1999).

[8] D. Reguera, J.M. Rubí, and L.L. Bonilla, Chapter 3 in Mathematical Modelling for Polymer Processing, (V. Capasso Ed., Springer-Verlag, in press).

[9] A. Ziabicki, J. Chem. Phys. 48, 4368 (1968).

[10] A. Ziabicki, J. Chem. Phys. 85, 3042 (1986).

[11] P.R. ten Wolde, M.J. Ruiz-Montero, and D. Frenkel, J. Chem. Phys. 104, 9932 (1996) and references quoted therein.

[12] P.R. ten Wolde, M.J. Ruiz-Montero, and D. Frenkel, Faraday Discuss. 104, 93 (1996).

[13] M. Avrami, J. Chem. Phys. 7, 1103 (1939); ibid 8, 212 (1940); ibid 9, 177 (1941). 
[14] A.N. Kolmogorov, Bull. Acad. Sci. USSR Mat. Ser. 1355 (1937).

[15] U.W. Gedde, Polymer Physics, (Chapman\&Hall, London, 1995).

[16] A.J. Ryan et. al., Faraday Discuss. 112, 13 (1999). 


\section{Conclusions and Perspectives}

In this thesis we have been mainly concerned with the study of the kinetics of nucleation processes, which constitute the first step in many phase transformations. Recent results obtained from experiments and simulations are increasingly evidencing that an accurate description of nucleation cannot be accomplished without accounting for the nonequilibrium aspects of the process.

Along the contents of the thesis, we have presented and developed a mesoscopic nonequilibrium description of the nucleation kinetics. The theory we have introduced provides a consistent and general framework allowing a flexible description of the process in terms of the relevant parameters. Our scheme is able to recover and complement the result of previous approaches, overcoming some of their drawbacks. Moreover, we have established the basis for different descriptions of the process in terms, for instance, of order parameters or of density functionals, which are promising alternatives to analyze the phenomenon in more detail. Besides, the framework we have presented sets up a link between theoretical approaches to homogeneous nucleation, generally formulated in terms of a kinetic equation of the Fokker-Planck type, and simulations, which mostly employ linear response theory. In this context, our scheme offers a theoretical framework to interpret and extend the results obtained in recent simulations, since it provides explicit and simulable expressions for the quantities and coefficients involved in the process.

The theory we have presented has confirmed its usefulness to clarify the mesoscopic subtleties of the process and to reveal potential nonequilibrium influences, in different circumstances. One of these cases refers to the proper meaning and scope of the thermodynamic relation known as "nucleation theorem". The validity and application of this thermodynamic relation to an unstable and small object such as the nucleus has motivated some concerns. We have demonstrated that the validity of the nucleation theorem transcends the phenomenon of nucleation and extends to all equilibrium systems containing local nonuniform density distributions stabilized by external fields. Moreover, 
it remains valid down to the molecular level. These results have been confirmed by some examples concerning cavities and clusters in hard rod fluids, as well as the molecular excesses associated with the "atmospheres" of molecules in single fluids. We have shown that, at the molecular level, the theorem can be associated with the compressibility equation of state and, at the macroscopic level, with the Gibbs adsorption equation. It is thus a relation of great power and should be useful in many contexts.

Another topic which has been subjected to intense controversy is the proper consideration of the degrees of freedom that the nucleating cluster possesses. The approach to this problem from an equilibrium perspective gave rise to the long-standing "translational-rotational paradox". In Chapter 4, we have clarified the paradoxical aspects that the inclusion of these degrees of freedom has presented through a mesoscopic analysis of the problem. In addition, the role that translational-rotational degrees of freedom plays in nucleation theories has been reconsidered by the proper accounting for the effects of motion of clusters in nucleation rate. Contrarily to the theories proposed up to now, we have adopted a non-equilibrium description more proper for a problem intrinsically out of equilibrium as nucleation is. In this sense, we have shown that when considering the process retaining the dynamics of the clusters a new, important, and genuine nonequilibrium correction to the nucleation kinetics arises.

The analysis of nonequilibrium effects in the process has also been treated in the subsequent chapter. One does not have to forget that nucleation occurs in a metastable phase and that the dynamics of this phase may strongly affect the process. Chapter 5 has been precisely devoted to analyze the influence of the medium. Apart from developing a general framework to describe nucleation in inhomogeneous situations, we have analyzed in detail three particularly interesting situations: nucleation in spatially inhomogeneous conditions, subjected to a temperature gradient or in the presence of a flow. This last situation is specially relevant since it has been scarcely analyzed in the literature despite its significant importance in many real nucleation processes, such as for instance the processing and crystallization of polymer melts. We have studied in detail the influence of the medium in real nucleation experiments in diffusion chambers, and we have also discussed the potential importance of these effects in polymer crystallization. The results confirm that actual nucleation experiments are not strongly affected by the medium at high pressures, but they suggest that this influence may have important consequences when pressure decreases, as occurs in atmospheric processes, and in polymer crystallization.

Finally, we have outlined how the results we have obtained for nucleation can settle the basis for a more realistic description of the next stage in the 
dynamics of the phase transformation: the growth of the nucleated regions up to fill the available space.

The range of application of our theory is by no means limited to these few particular cases. It constitutes a general framework in which, following systematically a small set of simple rules, one can derive kinetic equations for nucleation phenomena aiming to reproduce actual conditions and their influence in the process. Thus, the flexibility of the formalism we have presented may become very useful to the systematic description of more complicated situations. In addition, the intrinsic generality and multidimensional character of our theory facilitates its extension to other problems of interest.

In this sense, the theory we have introduced may open up new an interesting research lines to carry out future investigations. Concerning these perspectives, we can quote some short-term objectives and more wide generalizations.

The first objective we will pursue is the comparison of the predictions of our theory with the brand-new simulation results of Auer and Frenkel [1]. This work reports the first parameter-free estimation of the nucleation rate in a hard sphere colloid by means of computer simulations. The fact of using hard-spheres facilitates the comparison, since this system has been thoroughfully studied in the literature and its properties are available and well-known. It could be extremely important to test if our scheme can reproduce the simulation results and shed some light about the significant discrepancies between the results of these simulations, the predictions of the classical nucleation theory and the experimental results.

Other immediate application of our work concerns the results of Chapter 3. Now that the basis and proper meaning of the nucleation theorem have been firmly established, it becomes a valuable tool not only to infer information from experiments, but also to improve nucleation theories. In fact, the scaling relations proposed by McGraw and Laaksonen [2] have demonstrated that the systematic discrepancies of the classical nucleation theory with experiments can be parametrized by using this theorem. We are convinced that the nucleation theorem can settle the basis not only to account for the disagreements of CNT, but also to construct a more accurate phenomenological model for the free energy of formation of a cluster.

In addition, as we mentioned at the concluding remarks of that chapter, the generality of the theorem makes it a powerful tool to investigate a wider range of phenomena beyond the scope of nucleation. In particular, we are exploring the possibility of its application to the theory of the hydrophobic bond [3, 4], but there are many other possibilities.

Another topic of major interest which has not been discussed in the contents 
of the thesis is the definition of temperature of a cluster and the role played by thermal fluctuations in the nucleation rate [5]-[8]. The assignment of a temperature to small objects, and the proper conception of temperature fluctuations are very controversial issues in nucleation and in a general context [9]-[13]. Our theory, due to its intrinsic mesoscopic and nonequilibrium nature, starts off with advantage in elucidate the proper influence of temperature fluctuations in nucleation. In particular, the concepts and results discussed in Chapter 4 can be very useful in the clarification of this issue, and work along this lines is in progress.

Finally, the last immediate application of our theory we are now considering is to go deeply into the extension to account for other external influences in the nucleation process. These external influences may facilitate the induction and control over the process, for instance through external electric fields [14]-[16], ions [17]-[19] or pressure waves. The interplay of thermal noise, responsible for the overcoming of the nucleation barrier, with these external influences may give rise to resonant phenomena [20] which can be very useful to optimize and control the nucleation process. By merely introducing the proper additional variables in our formalism, which can be easily done due to its multidimensional character, we could provide a theoretical description of these situations.

In a more general outlook, our results can be extended in many directions. Specially interesting is the task of providing a more complete description of the cluster including its shape. This would be useful to analyze accurately nucleation in polar fluids [21] or crystallization [22], where the sharp spherical shape assumed in the classical approach have turned out to be insufficient. Another particularly relevant situation would be the extension of our theory to the case of binary or ternary systems, which are specially important in atmospheric processes [23]. The application of our formalism to heterogeneous nucleation would be of great interest as well.

In addition, it would be worth to pursue in more deep the hydrodynamic description introduced in the second chapter. Density functional theories are one of the most promising techniques to accomplish a more realistic description of the equilibrium facet of nucleation. The extension of the hydrodynamic characterization to non-conservative models would also constitute an issue of remarkable relevance.

Exploring the consequences of the formalism introduced in Chapter 6 on what concerns the calculation and prediction of new scaling exponents for the evolution of crystallinity, and its comparison with the experimental results [24], is an interesting perspective, too. The extension to open systems, including interaction between clusters, in the way outlined at the end of that chapter 
would overcome some of the limitations of the present nucleation theories.

Finally, due to the multidisciplinary character of the process, it would be also worth to investigate if the results we have presented here may have relevant implications to the other areas mentioned at the introduction, where nucleation is a very important topic.

In summary, despite all the significant efforts and the interdisciplinary interest in the field of nucleation, several fundamental questions remain opened, whose future answers envision interesting consequences. By achieving a greater understanding of nucleation, we could control the process and potentially change the course of many first-order phase transitions. 


\section{Bibliography}

[1] S. Auer and D. Frenkel, Nature 409, 1020 (2001).

[2] R. McGraw and A. Laaksonen, Phys. Rev. Lett. 76, 2754 (1996).

[3] A.B. Kolomeisky and B. Widom, Faraday Discuss. 112, 81 (1999).

[4] G.T. Barkema and B. Widom, J. Chem. Phys. 113, 2349 (2000).

[5] I.J. Ford and C.F. Clement, J. Phys. A: Math. Gen. 22, 4007 (1989).

[6] J.C. Barret, C.F. Clement, and I.J. Ford, J. Phys. A: Math. Gen. 26, 529 (1993).

[7] R. McGraw and R.A. LaViolette, J. Chem. Phys. 102, 8983 (1995).

[8] F.M. Kuni, A.P. Grinin, and A.K. Shchekin, Physica A 252, 67 (1998).

[9] R. McFee, Am. J. Phys. 41, 230 (1973).

[10] H. Fesbach, Phys. Today 40, 9 (1987).

[11] C. Kittel, Phys. Today 41, 93 (1988).

[12] B.B. Mandelbrot, Phys. Today 42, 71 (1989).

[13] J. Uffink and J. van Lith, Found. Phys. 29, 655 (1999).

[14] K.J. Oh, G.T. Gao, and X.C. Zeng, J. Chem. Phys. 109, 8435 (1998).

[15] G.T. Gao, K.J. Oh, and X.C. Zeng, J. Chem. Phys. 110, 2533 (1999).

[16] V.B. Warshavsky, T.V. Bykow, and X.C. Zeng, J. Chem. Phys. 114, 504 (2001).

[17] K.J. Oh, G.T. Gao, and X.C. Zeng, Phys. Rev. Lett.. 86, 5080 (2001). 
[18] J.H. Seinfeld, Science 288, 285 (2000).

[19] E.M. Knipping et al. Science 288, 301 (2000).

[20] T. Alarcón, Resonant Phenomena and Dissipation in Periodically Driven Systems, PhD thesis.

[21] P.R. ten Wolde, D.W. Oxtoby, and D. Frenkel, J. Chem. Phys. 111, 4762 (1999).

[22] S.-T. Yau and P.G. Vekilov, Nature 406, 494 (2000).

[23] A. Laaksonen, V. Talanquer, and D.W. Oxtoby. Annu. Rev. Phys. Chem. 46, 489 (1995).

[24] M.L. Di Lorenzo and C. Silvestre, Prog. Polim. Sci. 24, 917 (1999). 


\section{List of Publications}

- Fokker-Planck equations for nucleation processes revisited

D. Reguera, J.M. Rubí, and A. Pérez-Madrid

Physica A 259, 10 (1998).

- Kramers-type picture for crystal nucleation

D. Reguera, J.M. Rubí, and A. Pérez-Madrid

Journal of Chemical Physics 109, 5987 (1998).

- Controlling anomalous stresses in soft field-responsive systems

D. Reguera, J.M. Rubí, and A. Pérez-Madrid Physical Review E 62, 5313 (2000).

- Rotational motion and rheology of solid bodies in magnetic fluids

M.C. Miguel, D. Reguera, and J.M. Rubí

Magnetohydrodynamics 36, 327 (2000).

- Diffusion in stationary flow from mesoscopic nonequilibrium thermodynamics

I. Santamaría-Holek, D. Reguera, and J.M. Rubí

Physical Review E 63, 051106 (2001).

- A theorem for inhomogeneous systems: The generalization of the nucleation theorem

R.K. Bowles, D. Reguera, Y. Djikaev, and H. Reiss

Journal of Chemical Physics 115, 1853 (2001).

- Nonequilibrium translational-rotational effects in nucleation

D. Reguera and J.M. Rubí

Journal of Chemical Physics 115, 7100 (2001). 
- Kinetic equations for diffusion in the presence of entropic barriers

D. Reguera and J.M. Rubí

Physical Review E 64, 061106 (2001).

- Kinetic theory of nucleation and growth in polymers

D. Reguera, J.M. Rubí, and L.L Bonilla

Chapter 3 in the monograph on "Mathematical Modeling for Polymer Processing" (V. Capasso, Ed., Springer-Verlag) (in press).

- Mesoscopic approach to the slow dynamics of supercooled liquids and colloidal systems

A. Pérez-Madrid, D. Reguera, and J.M. Rubí

Journal of Physics: Condensed Matter (in press).

- Interplay of frequency-synchronization with noise: current resonances, giant diffusion and diffusion-crests

D. Reguera, P. Reimann, P. Hänggi, and J.M. Rubí

Europhysics Letters (in press). 